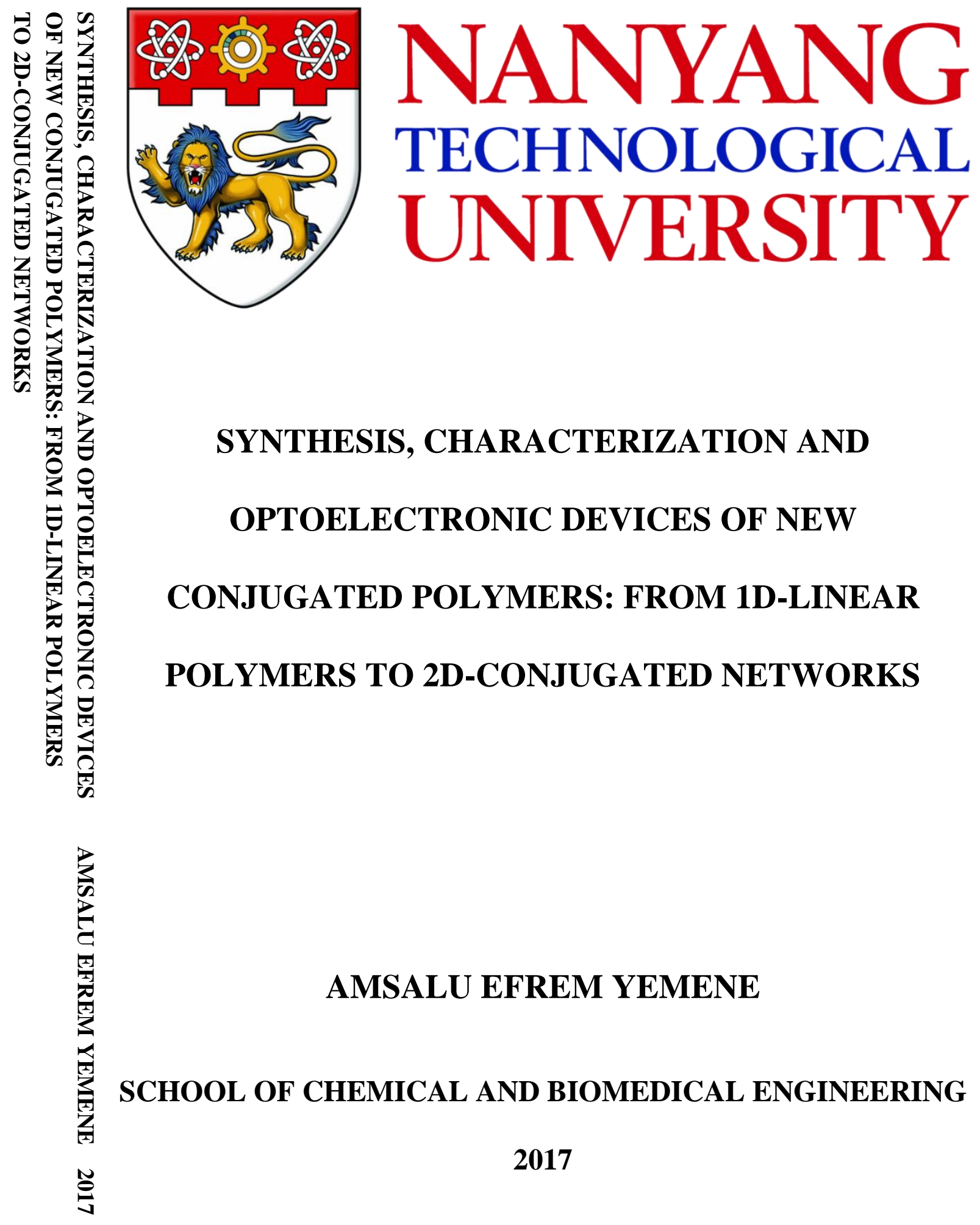




\section{SYNTHESIS, CHARACTERIZATION AND}

OPTOELECTRONIC DEVICES OF NEW

CONJUGATED POLYMERS: FROM 1D- LINEAR

POLYMERS TO 2D-CONJUGATED NETWORKS

\section{AMSALU EFREM YEMENE}

SCHOOL OF CHEMICAL AND BIOMEDICAL ENGINEERING

A thesis submitted to the Nanyang Technological University in partial fulfillment of the requirement for the degree of

Doctor of Philosophy 


\section{Acknowledgements}

First and foremost I praise God for the strength upon all who assisted me. I praise Him for giving me life, health, and courage to press on challenges and shortcomings I experienced along the journey of my Ph.D. research.

I would like to express my heartfelt sincere gratitude to my supervisors, Prof. Wang Mingfeng and Prof. NG Siu Choon (for the first part of my Ph.D. projects), for their invaluable and enthusiastic guidance, inspirational insights and helpful thoughts throughout all my Ph.D. research projects. They have encouraged and motivated me during my four years research work and I have learned a great deal of scientific knowledge from them all.

I would like to acknowledge all my lab mates both past and present especially Lu Yong (Dr.), Jia Tao (Dr.), Wang Kai, Yang Cangjie, Huang Shuo and Hassan Bohra for their help on my projects, kind collaborations, and intellectual discussions and also for their warm friendship and exchange of ideas we enjoy.

Thanks also go to the Singapore International Graduate Award (SINGA) and the School of Chemical and Biomedical Engineering, Nanyang Technological University (NTU) for providing me all the financial support during my $\mathrm{Ph} . \mathrm{D}$. studies.

To my friends, Biruhalem Taye, Abrha Gebregziabher, and Prince Nana Amaniampong (Dr.) thank you for offering me advice, the debates, dinners, general

help, and friendship were all greatly appreciated. To my friends scattered around the 
world, Solomon Legesse (Dr.), and Desalegn Alemu (Dr.) thank you for your thoughts, well wishes/ prayers, phone calls, e-mails, texts, visits, and being there whenever I needed help.

Last, but not least, I would like to thank my parents, especially my dad Mr. Efrem Yemene and my mom Mrs. Worknesh Desta, my sisters (Lemlem, Eden, Etsegent, Elsa) and brothers (Zelalem and Samson), who have been there to let me feel how home could be so near yet so far and for their prayers, understanding, boundless support and encouragement to achieve my goals.

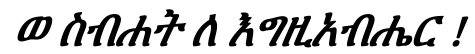




\section{Table of Content}

Acknowledgements................................................................. i

Table of contents ....................................................................iii

List of Figures.............................................................viii

List of Schemes...............................................................xv

List of Tables..............................................................xvii

Abstract......................................................................xix

\section{Table of Contents}



1.1. Conjugated Polymers ....................................................................................2

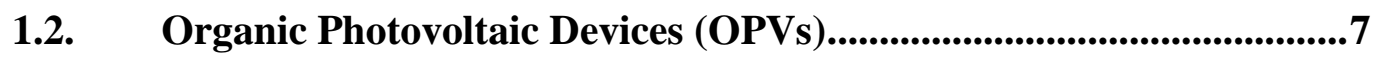

1.2.1. Introduction .............................................................................. 7

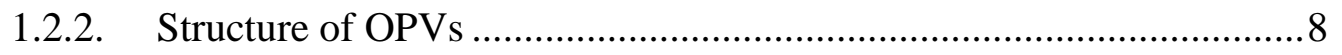

1.2.3. Operation Principles of OPV s..................................................... 9

1.2.4. Device Architectures of OPVs .................................................... 10

1.2.5. Design strategy towards high efficiency OPVs materials ................. 14

1.3. Organic Field Effect Transistors (OFETs) ...........................................17

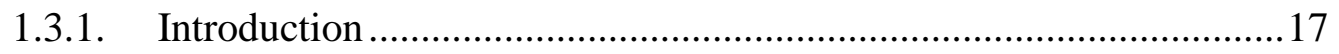

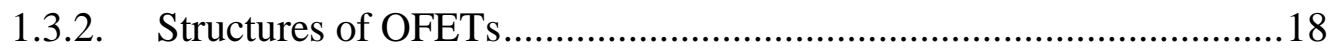

1.3.3. Operation Principles of OFETs ...................................................... 19

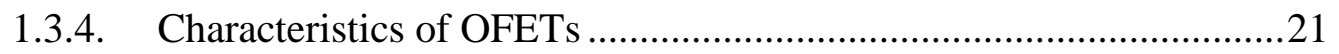

1.3.5. Conjugated polymer materials design for OFET devise ...................22

1.4. Synthetic Route of Conjugated Polymers .............................................24 


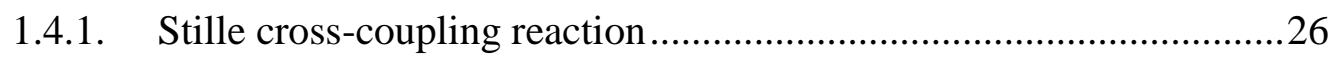

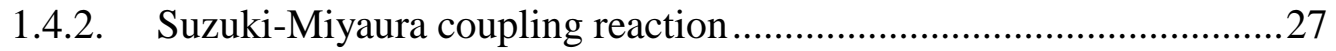

1.4.3. Direct $\mathrm{C}-\mathrm{H}$ arylation coupling reaction ......................................2 28

1.5. Donor-Acceptor Conjugated Polymers for Organic Electronics Applications (OPVs and OFETs) .............................................................................31

1.6. Motivation and Scope of the Thesis.................................................39

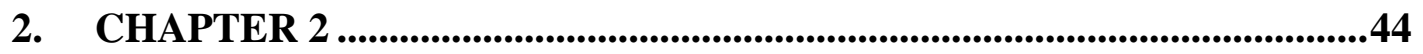

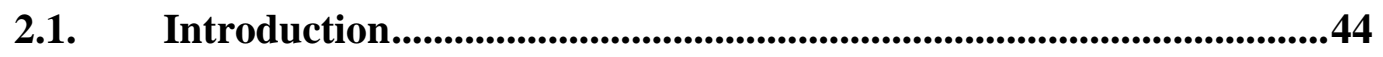

2.2. Experimental .................................................................................................48



2.2.2. Synthesis of polymer P1, P2, P3, PF-S, PF-O …..........................61

2.2.3. GPC traces for polymers and NMR spectra................................66

2.3. Results and Discussion .................................................................77

2.3.1. Synthesis of monomers and D-A polymers ..................................77

2.3.2. Thermal Properties ................................................................. 81



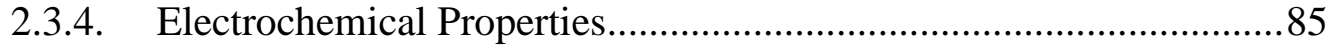

2.3.5. Photovoltaic Properties of P1, P2, P3, PF-S, and PF-O Polymers .....87

2.3.6. Field Effect Transistor Properties of P1, P2, P3, PF-S, and PF-O Polymers ..................................................................................... 91

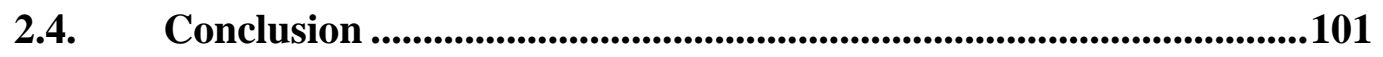



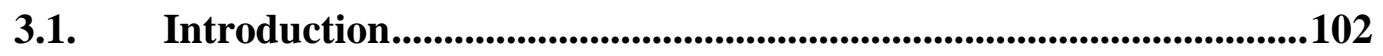

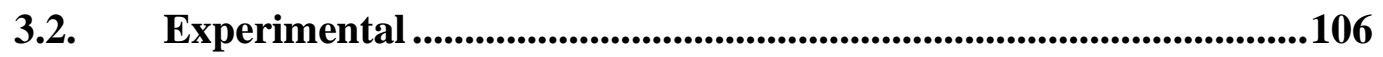

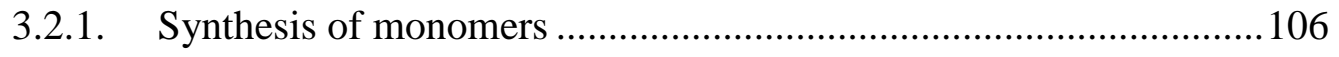

3.2.2. Synthesis of polymer P5 and P6 …........................................ 108

3.2.3. GPC traces for polymers and NMR spectra ............................... 110 
3.3. Results and Discussion

3.3.1. Synthesis of monomers and polymers...............................................113

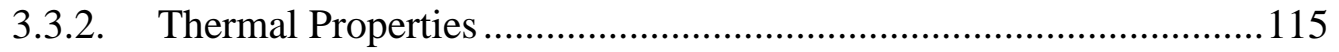

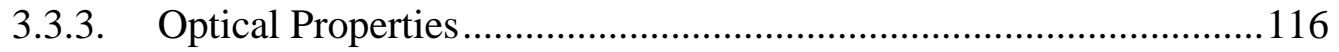

3.3.4. Electrochemical Properties and DFT Calculation ............................. 120

3.3.5. Field-Effect Transistor Devices ..................................................... 123

3.4. Conclusion ....................................................................................................129

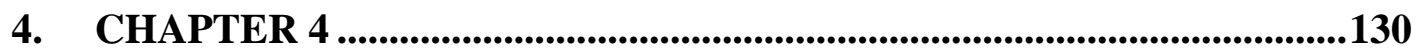

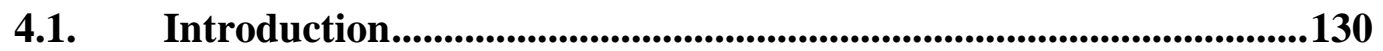

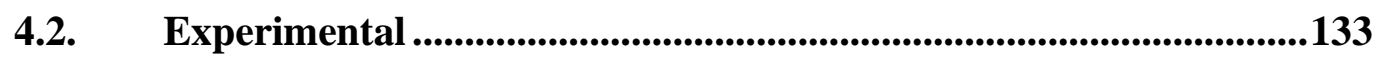

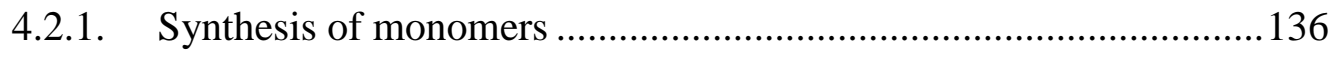

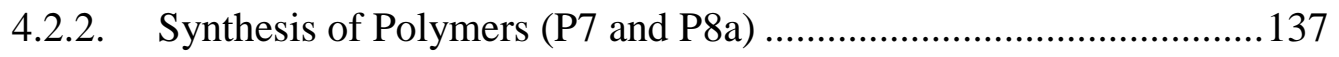

4.2.3. GPC traces for polymers and NMR spectra .................................... 138

4.3. Results and Discussion ............................................................................140

4.3.1. Synthesis of Monomers and Polymers (P7 \&P8) .............................. 140

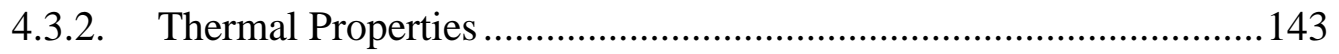

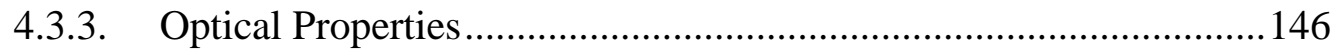

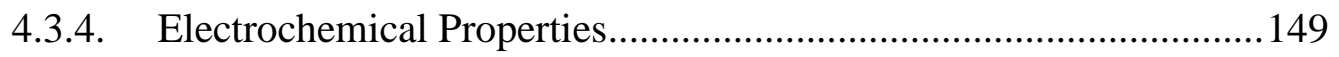

4.3.5. Field-Effect Transistor Devices ...................................................... 151



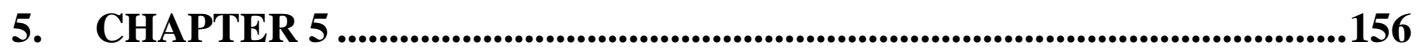

5.1. Introduction................................................................................156

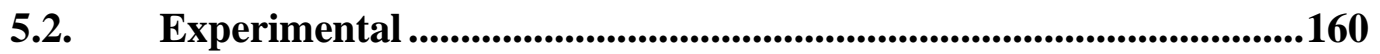

5.2.1. General synthesis procedure of Compound 22 via direct $\mathrm{C}-\mathrm{H}$ arylation couplings ............................................................. 161

5.2.2. General synthesis procedure for PDFBT-Th 4 polymer via direct

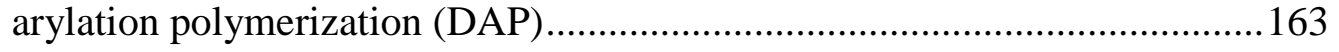

5.2.3. Synthesis of PDFBT-Th4 polymer via Stille polymerization............ 165 
5.2.4. GPC traces for polymers and NMR spectra.....................................166

5.3. Results and Discussion ..............................................................................169

5.3.1. Synthesis and structural characterization of PDFBT-Th4 polymer.. 169

5.3.2. Thermal Properties of PDFBT-Th 4 Polymer....................................... 175

5.3.3. Optical Properties of PDFBT-Th4 polymer.......................................176

5.3.4. Electrochemical properties of PDFBT-Th 4 polymer.......................... 180

5.3.5. Field-effect transistor properties of PDFBT-Th 4 polymer .................182

5.3.6. Photovoltaic Properties PFFBT-Th ${ }_{4}$ Polymers .................................. 188

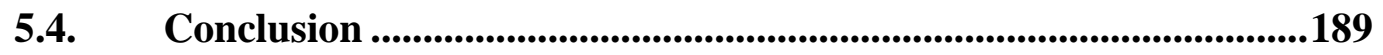

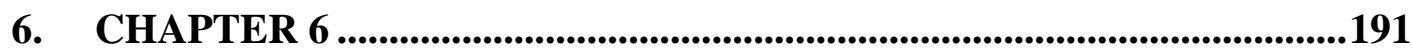

6.1. Introduction.......................................................................................................191

6.2. Experimental .............................................................................................195

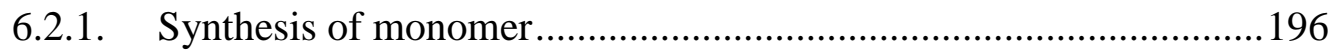

6.2.2. General synthesis procedure for PDFBT-DTP polymer via direct arylation polymerization (DAP) .................................................................... 197

6.2.3. GPC traces for polymers and NMR spectra...................................... 199

6.3. Results and Discussion ...........................................................................202

6.3.1. Synthesis and structural characterization .........................................202

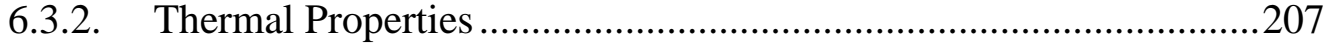

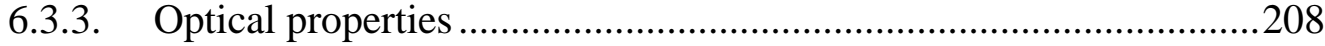

6.3.4. Electrochemical properties.............................................................211

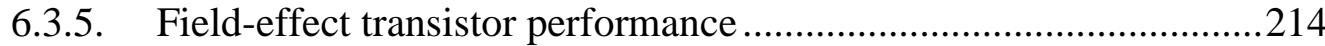

6.4. Conclusion ...............................................................................................217

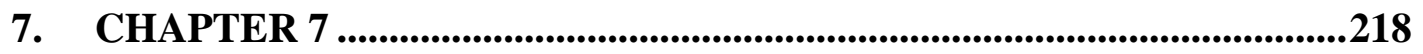

7.1. Introduction................................................................................218

7.2. Experimental ...................................................................................................220




7.2.2. General synthetic protocol for dithieno[3,2-a:2',3'-c]phenazine based conjugated microporous organic polymer (DTP-CMP)............................222

7.2.3. NMR Spectra of monomer .........................................................224

7.3. Results and Discussion ..........................................................224

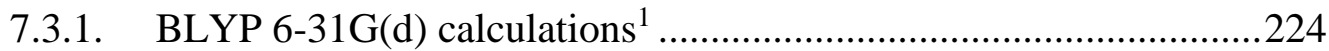

7.3.2. Synthesis of monomers and DTP-CMPs......................................22

7.3.3. Thermal properties of DTP-CMPs ..............................................2233

7.3.4. Optical and electrochemical properties .........................................2234

7.3.5. Porosity Characteristics of DTP-CMPs based on Brunauer-EmmettTeller (BET) measurements ......................................................................2237

7.3.6. Morphologies of the DTP-CMP polymers .................................241

7.4. Conclusion .................................................................................244

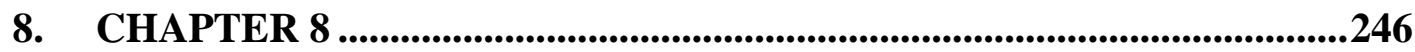

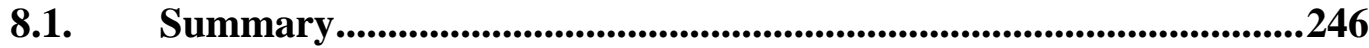

8.2. Suggestions for Future Work.........................................................250

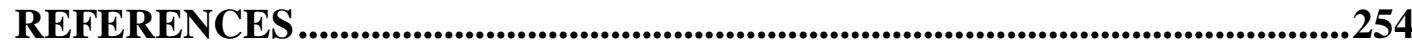

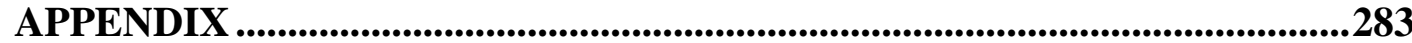

AI-1. Journal publications..............................................................................283

AI-2. Conference presentations ...................................................................284 


\section{List of Figures}

Figure 1.1. A schematic representation of energy gap in (a) insulator (b) semiconductor $(\mathrm{c})$ conductor............................................................................. 3

Figure 1.2. The molecular structure of polyacetylene $\left(\mathrm{C}_{2} \mathrm{H}_{2}\right)_{\mathrm{n}}$.........................5

Figure 1.3. The architecture of the conventional organic photovoltaic device.........8

Figure 1.4. The photoconversion mechanism of an OPV devices. ....................... 10

Figure 1.5. Schematic representation of bilayer heterojunction devices.................11

Figure 1.6. Schematic representation of inverted-BHJ OPV (a) and an energy level

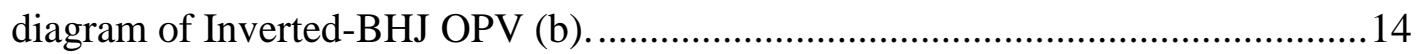

Figure 1.7. Illustration for the typical current-voltage (I-V) characteristics

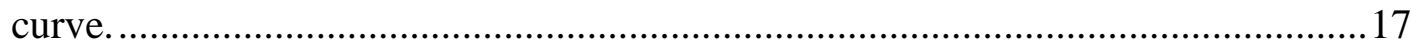

Figure 1.8. Four common types of OFETs structures top contact (a), (c) and bottom contact (b), (d) OFET devices.

Figure 1.9. Schematic representation of OFETs operating principles (a) Charge carriers (holes (+) or electron (-)) forming accumulation layer. (b) Charge flow....21 Figure 1.10. The schematic representation for a typical output (a) and transfer curves (b). 22

Figure 1.11. Examples of the chemical structure of p-type, n-type, and ambipolar semiconductors .24

Figure 1.12. Illustration for catalytic cycle of Palladium-catalyzed crosscoupling reactions. .26

Figure 1.13. Representative synthesis Scheme of $\pi$-conjugated polymer (PTB7) by Stille (a), BTz-Th3 by Suzuki-Miyaura (b), and TPD-Th3 by direct arylation coupling method (c). 30

Figure 1.14. PPV and its derivative type conjugated polymers for OPV applications 
Figure 1.15. Thiadiazole electron withdrawing group containing acceptor moieties. 35

Figure 1.16. Five member lactam-ring containing acceptor moieties 37

Figure 2.1. GPC traces for P1, P2, P3, PF-S and PF-O (using $\mathrm{CHCl}_{3}$ as eluent at 80 $\left.{ }^{\circ} \mathrm{C}\right)$ from RI (a, c, e, g, i) and UV (550 nm) (b, d, f, h, j) detectors, respectively. ...67 Figure 2.2. ${ }^{1} \mathrm{H}$ NMR (300 MHz) spectra for compound 1, 3, 4, 10, 11, 13a, 14a, 15a, 16a, 13b, 14b, 15b, and 16b (a, b, c, d, e, f, g, h, i, j, k, l, and m), respectively, in $\mathrm{CDCl}_{3}$ solvent at R.T. .74

Figure 2.3. ${ }^{1} \mathrm{H}$ NMR (300 MHz) spectra for $\mathbf{P 1}$ (a), $\mathbf{P 2}$ (b), $\mathbf{P 3}$ (c) in $\mathrm{C}_{2} \mathrm{D}_{2} \mathrm{Cl}_{4}$ solvent at $100{ }^{\circ} \mathrm{C}$ and PF-S (d), PF-O (e) in $\mathrm{CDCl}_{3}$ solvent at R.T. .77

Figure 2.4. TGA plots of the five D-A polymers with a heating rate of $10{ }^{\circ} \mathrm{C} \mathrm{min}-1$ under nitrogen. 81

Figure 2.5. UV-vis absorption spectra of D-A polymers (a) in chloroform solution and (b) thin films for D-A polymers, P1, P2, P3, PF-S, and PF-O. .84

Figure 2.6. The evolutions of UV-vis absorption spectra of $\mathbf{P 1}$ in $\mathrm{CHCl}_{3}$ during (a) heating process from 25 to $70{ }^{\circ} \mathrm{C}$ and (b) cooling process from 70 to $15^{\circ} \mathrm{C}$..........85 Figure 2.7. The cyclic voltammograms of P1, P2, P3, PF-S, and PF-O films on a platinum electrode measured in $0.1 \mathrm{~mol} / \mathrm{lit}^{\mathrm{Bu}} \mathrm{Nu}_{4} \mathrm{NPF}_{6}$ acetonitrile solutions at a scan rate of $50 \mathrm{mVs}^{-1}$ .86

Figure 2.8. Current density-voltage curves of photovoltaic devices with an active layer of D-A polymer/ $\mathrm{PC}_{71} \mathrm{BM}$ (1/2 by weight) and $3 \%$ of DIO additive at $1000 \mathrm{rpm}$ spin speed.

Figure 2.9. Current density-voltage curves of photovoltaic devices with active layers of P1, P2 , and $\mathbf{P 3}$ and $\mathrm{PC}_{71} \mathrm{BM}$ acceptor (1:2 by weight) with $3 \%$ of DIO additive at 500 rpm spin speed. .90

Figure 2.10. IPCE spectra of the OPV devices using P1, P2, and P3 as donor polymers and $\mathrm{PC}_{71} \mathrm{BM}$ as acceptor. 91 
Figure 2.11. Transfer characteristics, output curve, and hysteresis behaviors of OFET devices with D-A polymer channel semiconductors (on OTS-8-modified substrates, annealed at $120^{\circ} \mathrm{C}$ ). P1: (a), (b) and (c), respectively; P2: (d), (e), and (f), P3: (g, h, i), PF-S: (j, k, l), and PF-O: (m, n, o), respectively..............................96 Figure 2.12. Transfer characteristics, output curve, and hysteresis behaviors of OFET devices with D-A polymer channel semiconductors (on OTS-18-modified substrates, annealed at $\left.120^{\circ} \mathrm{C}\right)$. P1: (a), (b) and (c), respectively; P2: (d), (e) and (f), respectively; P3: $(\mathrm{g}, \mathrm{h}, \mathrm{i})$ 97

Figure 2.13. Tapping-mode AFM height images $\left(5 \times 5 \mu \mathrm{m}^{2}\right)$ of pristine polymer films on OTS-modified $\mathrm{Si} / \mathrm{SiO}_{2}$ substrates for all polymers, $\mathbf{P 1}(\mathrm{A}), \mathbf{P 2}(\mathrm{B}), \mathbf{P 3}(\mathrm{C})$, PF-S (D), PF-O (E) annealed at $120{ }^{\circ} \mathrm{C}$. 100

Figure 3.1. DFT calculation at B3LYP/6-31G(d,p) level for geometry, molecular orbital distribution, and HOMO/LUMO energy levels of $\gamma$-lactone-Pechmann dye (left) and $\gamma$-lactam-Pechmann dye (right). For computational simplicity, the alkyl chains are replaced with methyl groups...... 105

Figure 3.2. GPC traces for $\mathbf{P 5}$ and $\mathbf{P 6}$ (using $\mathrm{CHCl}_{3}$ as eluent at $80{ }^{\circ} \mathrm{C}$ ) from $\mathrm{RI}(\mathrm{a}$ and c) and UV (550 nm) detectors (b and d), respectively. 110

Figure 3.3. ${ }^{1} \mathrm{H}$ NMR (300 MHz) spectra for compound 17 and 18 (a and b), respectively, in $\mathrm{CDCl}_{3}$ solvent at R.T. 111

Figure 3.4. ${ }^{1} \mathrm{H}$ NMR (300 MHz) spectra for $\mathbf{P 5}$ (a) and $\mathbf{P 6}$ (b) in a $\mathrm{C}_{2} \mathrm{D}_{2} \mathrm{Cl}_{4}$ solvent at $100{ }^{\circ} \mathrm{C}$. .112

Figure 3.5. (a) TGA and (b) DSC traces at a heating rate of $10{ }^{\circ} \mathrm{C} \min ^{-1}$ under nitrogen. 116

Figure 3.6. UV-vis-NIR absorption spectra of polymers in chloroform and in thin films measured at room temperature (a) for P5 and (b) for P6. Temperature dependent absorption spectra of polymers in chlorobenzene solution (c) for P5 and (d) for $\mathbf{P 6}$. 119 
Figure 3.7. The cyclic voltammograms of $\mathbf{P 5}$ (a) and $\mathbf{P 6}$ (b) polymer films (c) Ferrocene measured in $0.1 \mathrm{~mol} / \mathrm{lit}_{\mathrm{Bu}} \mathrm{NPF}_{6}$ acetonitrile solutions at a scan rate of 50 $\mathrm{mVs}^{-1}$. 122

Figure 3.8. DFT calculation at B3LYP/6-31G(d,p) level for geometry, molecular orbital distribution, and HOMO/LUMO energy levels of P5 (left) and P6 (right). For computational simplicity, the alkyl chains are replaced with methyl groups

Figure 3.9. Typical transfer curves (a to d) and output characteristics curves (e to h) of $\mathbf{P 5}$ and $\mathbf{P 6}$ based OFET devices measured under the $\mathrm{N}_{2}$-filled glovebox. OFETs devices were prepared on OTS-18-modified $\mathrm{Si} / \mathrm{SiO}_{2}$ substrate $(\mathrm{W} / \mathrm{L}=10 \mathrm{~mm} / 5 \mu \mathrm{m}$ devices).

Figure 4.1. Illustration for methods of t-BoC removal and the possible hydrogen bond network

Figure 4.2. GPC traces for $\mathbf{P 7}$ and $\mathbf{P 8 a}$ (using $\mathrm{CHCl}_{3}$ as eluent at $80{ }^{\circ} \mathrm{C}$ ) from $\mathrm{RI}(\mathrm{a}$ and c) and UV (550 nm) (b and d) detectors, respectively. 139

Figure 4.3. ${ }^{1} \mathrm{H}$ NMR (300 MHz) spectra for compound 21 (a) in $\mathrm{CDCl}_{3}$ solvent at R.T. 139

Figure 4.4. ${ }^{1} \mathrm{H}$ NMR (300 MHz) spectra for $\mathbf{P 7}$ (a) in a $\mathrm{C}_{2} \mathrm{D}_{2} \mathrm{Cl}_{4}$ solvent at 100 ${ }^{\circ} \mathrm{C}$ 140

Figure 4.5. FT-IR spectra for P7 and P8a polymers. 143

Figure 4.6. TGA trace for P7 (a) and P8a (b) polymers. DSC traces for P7 (c) and P8a (d) polymers with a heating rate of $10{ }^{\circ} \mathrm{C} \mathrm{min}^{-1}$ under nitrogen 145

Figure 4.7. UV-vis spectra of $\mathbf{P 7}$ and P8a polymers in chloroform solution (a) and thin films (b). Chlorobenzene solution spectra of P8a at $80{ }^{\circ} \mathrm{C}$ and after cooling to r.t (c). 149

Figure 4.8. The cyclic voltammograms of P7 (a), P8a (b), and Ferrocene (c) films on a glassy carbon working electrode measured in $0.1 \mathrm{~mol} / \mathrm{lit}_{\mathrm{Bu}} \mathrm{NPF}_{6}$ acetonitrile solutions at a scan rate of $50 \mathrm{mVs}^{-1}$. 151 
Figure 4.9. Typical transfer curves (a to d) and output characteristics curves (e to g) for the P7 polymer.

Figure 5.1. GPC traces for PDFBT-Th4 (using THF as eluent at r.t.) from RI (a, c, e, and $\mathrm{g})$ and $\mathrm{UV}(550 \mathrm{~nm})(\mathrm{b}, \mathrm{d}, \mathrm{f}$, and $\mathrm{h})$ detectors, respectively. 167

Figure 5.2. ${ }^{1} \mathrm{H}$ NMR (300 MHz) spectra for compound 22 (a) and 23 (c); ${ }^{13} \mathrm{C}$ NMR (300 MHz) spectra for compound 22 (b) in $\mathrm{CDCl}_{3}$ solvent at R.T. 169

Figure 5.3. ${ }^{1} \mathrm{H}$ NMR spectra of the PDFBT-Th4 polymers synthesized via DAP, E3a (a), Stille-B1 (b), and Stille-B2 (c) at $100{ }^{\circ} \mathrm{C}$ in $\mathrm{C}_{2} \mathrm{D}_{2} \mathrm{Cl}_{4}$ 175

Figure 5.4. TGA plots of PDFBT-Th4 polymers with a heating rate of $10{ }^{\circ} \mathrm{C} \mathrm{min}-1$ under a nitrogen atmosphere. 176

Figure 5.5. UV-vis absorption spectra from $\mathrm{CHCl}_{3}$ solution (a) and from $\mathrm{CB}$ solution (b). Temperature dependent spectra for E3a, Stille-B1, and Stille-B2 polymers upon heating (c, e, \& g) and upon cooling (d, f, \& h), respectively. Thin film spectra for E3a, Stille-B1, and Stille-B2 polymers at r.t (i) and after annealing at $100{ }^{\circ} \mathrm{C}$ (j) of PDFBT-Th4 polymers. 179

Figure 5.6. Cyclic voltammetry curve for PDFBT-Th4 polymers (a) for E3a and (b) for Stille-B1 (c) for Stille-B2 (inset CV is for ferrocene standard) 182

Figure 5.7. Typical transfer curves (a to f) and output characteristics curves ( $\mathrm{g}$ to $\mathrm{k}$ ) for PDFBT-Th4 polymers. 186

Figure 5.8. AFM height $\left(2 \times 2 \mu \mathrm{m}^{2}\right)$ images for PFFBT-Th4 polymers E3a, E5 and Stille-B1 polymers (a, b, c: at R.T) and (d, e, f: after annealing at $100{ }^{\circ} \mathrm{C}$ ), respectively.

Figure 5.9. Current density-voltage curves of photovoltaic devices with active layers of PDFBT-Th4 (E3a-DAP and Stille-B2 polymers) and $\mathrm{PC}_{71} \mathrm{BM}$ acceptor (1:1.2 by weight) with $3 \%$ of DIO additive at $800 \mathrm{rpm}$ spin speed. 189

Figure 6.1. Chemical structures of BDT, CPDT, DTS, DTG, and DTP units. R = alkyl chains 193 
Figure 6.2. GPC traces for PDFBT-DTP polymers of E1-DTP, E2-DTP, E3-DTP, E5-DTP, E6-DTP of $\mathrm{CHCl}_{3}$ frac. and E6-DTP of CB frac. (using THF as eluent at r.t.) from RI (a, c, e, g, i, and k) and UV (550 nm) (b, d, f, h, j, and l) detectors, respectively. 201

Figure 6.3. ${ }^{1} \mathrm{H} \mathrm{NMR}(300 \mathrm{MHz})$ spectra for compound $\mathbf{2 4}(\mathrm{a})$ and 26 (b) in $\mathrm{CDCl}_{3}$ solvent at R.T. 201

Figure 6.4. ${ }^{1} \mathrm{H}-\mathrm{NMR}$ spectra of the PDFBT-DTP polymers synthesized via DAP, E1-DTP (a), E2-DTP (b), E3-DTP (c), E5-DTP (d), and E6-DTP-CB (d) at $100{ }^{\circ} \mathrm{C}$

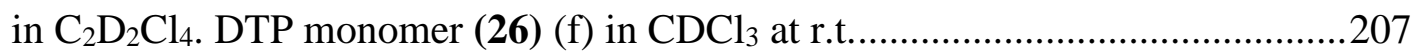
Figure 6.5. TGA plots of PDFBT-DTP (Entry E2-DTP) polymer with a heating rate of $10{ }^{\circ} \mathrm{C} \mathrm{min}-1$ under nitrogen. 208

Figure 6.6. UV-vis-NIR absorption spectra of PDFBT-DTP polymers (a) in $\mathrm{CHCl}_{3}$ solution except for E6-DTP (2) in chlorobenzene solution and (b) in the thin film prepared by spin casting from their chlorobenzene solution. E6-DTP (1) is $\mathrm{CHCl}_{3}$ fraction and E6-DTP (2) is chlorobenzene fraction.

210

Figure 6.7. Cyclic voltammetry curve for PDFBT-DTP polymers: E1-DTP (a), E2DTP (b), E3-DTP (c), E5-DTP (d), E6-DTP (1) of $\mathrm{CHCl}_{3}$ fraction (e), E6-DTP (2) of chlorobenzene fraction (f) and ferrocene standard (g) 214 Figure 6.8. Transfer curves of E2-DTP polymer (a) at r.t and (b) after annealing at $100{ }^{\circ} \mathrm{C}$. Output characteristics curve of E2-DTP polymer for after annealing at 100 ${ }^{\circ} \mathrm{C}(\mathrm{c})$. 216

Figure 6.9. AFM height $\left(5 \times 5 \mu \mathrm{m}^{2}\right)$ images for PDFBT-DTP polymer of E2-DTP at R.T. (a) and after annealing at $100{ }^{\circ} \mathrm{C}$ (b). 216

Figure 7.1. ${ }^{1} \mathrm{H} \mathrm{NMR}(300 \mathrm{MHz})$ spectra for compound 26(a) in $\mathrm{CDCl}_{3}$ solvent at R.T .224

Figure 7.2. Representative geometry-optimized structure by DFT (BLYP 6-31G(d)) for DBDTP monomer, linear tetramer $\left(\mathbf{L}_{4}\right)$, cyclic tetramer $\left(\mathbf{C}_{4}\right)$, and an extended conjugated network $(\mathbf{C} 9)$. 
Figure 7.3. FT-IR spectra of DTP-CMPs in comparison to the monomer (DBDTP) (a) zoomed in the range of 400-2200 $\mathrm{cm}^{-1}$ (b)............................231

Figure 7.4. ${ }^{1} \mathrm{H}$ NMR spectra (a) DBDTP monomer in $\mathrm{C}_{2} \mathrm{D}_{2} \mathrm{Cl}_{4}$ at r.t., and (b) CMP-



Figure 7.5. TGA plots of DTP-CMP polymers with a heating rate of $10{ }^{\circ} \mathrm{C} \mathrm{min}-1$

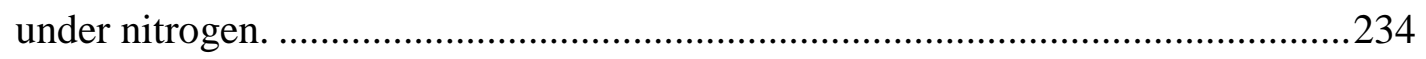

Figure 7.6. UV-vis-NIR absorption spectra of monomer (DBDTP) and DTP-CMP

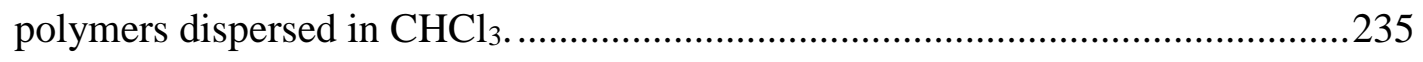

Figure 7.7. Cyclic voltammograms of DTP-CMP polymers. ..............................236

Figure 7.8. Nitrogen gas adsorption-desorption isotherms of DTP-CMP porous

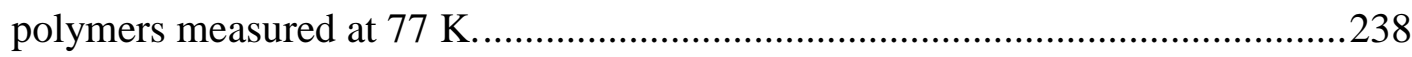

Figure 7.9. Pore size distribution profiles for DTP-CMP porous polymers. ........240

Figure 7.10. Representative SEM images for CMP-NTU1 (a, b), CMP-NTU2 (c, d), CMP-NTU3 (e, f), and CMP-NTU4 (g, h). Red arrows indicate the tube-like



Figure 7.11. TEM images for DTP-CMP for CMP-NTU1 (a), CMP-NTU2 (b),

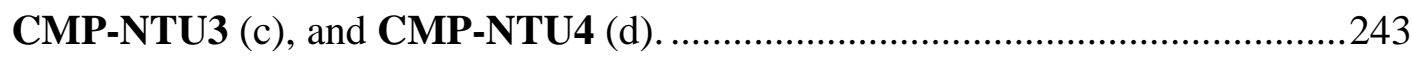

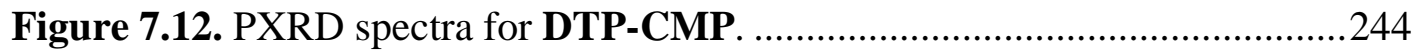




\section{List of Schemes}

Scheme 1: Synthesis of intermediate 4 and 5: (i) $\mathrm{PCC}, \mathrm{CH}_{2} \mathrm{Cl}_{2}$, r.t, 90\%. (ii) $i$ $\operatorname{PrMgCl} \cdot \mathrm{LiCl}$, THF, r.t. (iii) $\mathrm{LiAlH}_{4} / \mathrm{AlCl}_{3}, \mathrm{Et}_{2} \mathrm{O}$, r.t, 50\% (iv) NBS, DMF, r.t, $80 \%$. (v) $\mathrm{Sn}(\mathrm{Me})_{3} \mathrm{Cl}, \mathrm{THF},-78{ }^{\circ} \mathrm{C}, 96 \%$ .46

Scheme 2: Synthesis of donor moieties, Compound 8 and 9. (vi) $\mathrm{Pd}_{2}(\mathrm{dba})_{3} / \mathrm{P}(\mathrm{o}-$ tolyl) ${ }_{3}$, THF, reflux, $44 \%$ for 6 and $41 \%$ for 7. (vii) (Me) ${ }_{3} \mathrm{SnCl}$, THF, r.t, $88 \%$ for 8 and $70 \%$ for 9. .47

Scheme 3: Synthesis of acceptor monomers 16a and 16b. (i) $\mathrm{n}-\mathrm{BuLi},-78{ }^{\circ} \mathrm{C}$, $\mathrm{CuBr} / \mathrm{LiBr}$ then oxalyl chloride, THF, 78\%. (ii) $\mathrm{FeCl}_{3}, \mathrm{CH}_{2} \mathrm{Cl}_{2}$, r.t, 82\%. (iii) 13a) $\mathrm{HONH}_{2} \cdot \mathrm{HCl}, 10 \% \mathrm{Pd} / \mathrm{C}, 78{ }^{\circ} \mathrm{C}$ then $\mathrm{N}_{2} \mathrm{H}_{4} \cdot \mathrm{H}_{2} \mathrm{O}, \sim 60{ }^{\circ} \mathrm{C}, \mathrm{EtOH}, 34 \%$. (iv)13a) $\mathrm{SOCl}_{2}, \mathrm{Et}_{3} \mathrm{~N}, \mathrm{DCM}$, reflux, 79\%. (iii) $\left.13 \mathrm{~b}\right) \mathrm{HONH}_{2} \cdot \mathrm{HCl}, 85^{\circ} \mathrm{C}$ for $24 \mathrm{~h}$. (iv) $\mathbf{1 3 b}$ ) $140{ }^{\circ} \mathrm{C}$ for $60 \mathrm{~h}, \mathrm{EtOH}, 30 \%$. (v) $\mathrm{Br}_{2}, \mathrm{CHCl}_{3}$, reflux, $75 \%$ for $\mathbf{1 4 a}$ and $69 \%$ for $\mathbf{1 4 b}$. (vi) $\mathrm{Pd}\left(\mathrm{PPh}_{3}\right)_{4}$, toluene, $110{ }^{\circ} \mathrm{C} .71 \%$ for $\mathbf{1 5 a}$ and $84 \%$ for $\mathbf{1 5 b}$ (vii) NBS, THF, r.t. $70 \%$ for $\mathbf{1 6 a a n d} 92 \%$ for $\mathbf{1 6 b}$. 47

Scheme 4: Previous synthetic route to D-A polymers, P1 and P2. (i) $\mathrm{Pd}\left(\mathrm{PPh}_{3}\right)_{4}$, toluene, $110^{\circ} \mathrm{C}, 54.5 \%(\mathbf{P 1})$ and $27 \%(\mathbf{P 2})$ yield. 48

Scheme 5: Improved synthetic route to D-A polymers, P1, P2, P3, PF-S and PF-O. (i) $\mathrm{Pd}\left(\mathrm{PPh}_{3}\right)_{4}$, tolune, $110{ }^{\circ} \mathrm{C}, 68 \%$ (P1), 47\% (P2), 41\% (P3). (ii) $\mathrm{Pd}\left(\mathrm{PPh}_{3}\right)_{4}$, tolune, $2 \mathrm{M} \mathrm{K}_{2} \mathrm{CO}_{3}, 110{ }^{\circ} \mathrm{C}, 70 \%$ (PF-S) and $82 \%$ (PF-O) .79

Scheme 6: Synthetic route to Compound 19 (Br2-Th-Pech). (i) $\mathrm{AlCl}_{3}, \mathrm{CH}_{2} \mathrm{Cl}_{2}, \mathrm{R}$.T., $18 \mathrm{~h}, 47 \%$ yield. (ii) $\mathrm{CuCl}, \mathrm{NH}_{4} \mathrm{Cl}, \mathrm{Ac}_{2} \mathrm{O}, 140{ }^{\circ} \mathrm{C}, 2 \mathrm{~h}, 34 \%$ yield. (iii) $\mathrm{NBS}, \mathrm{CH}_{2} \mathrm{Cl}_{2}$, R.T., 24 h, 24\% yield. 114

Scheme 7: Synthetic route to $\mathbf{P 5}$ and $\mathbf{P 6}$ polymers via Stille coupling. (i) $\mathrm{Pd}_{2}(\mathrm{dba})_{3}$, $\mathrm{P}(\text { o-tol })_{3}$, tolune, $24 \mathrm{~h}, 110^{\circ} \mathrm{C}, 78.8 \%$ (P5) and $90{ }^{\circ} \mathrm{C}, 86 \%$ (P6) yield. 115

Scheme 8: Synthesis of P7 polymer via Stille polymerization method and t-BoC deprotection by using chemical method (P8a) and thermal treatment method (P8b). (i) $\mathrm{AcOH}, \mathrm{HCl}$, reflux, $24 \mathrm{~h}, 100 \%$ yield. (ii) DMAP, $\mathrm{Et}_{3} \mathrm{~N}, \mathrm{BoC}_{2} \mathrm{O}, \mathrm{THF}, 24 \mathrm{~h}, 84 \%$ 
yield. (iii) $\mathrm{Pd}\left(\mathrm{PPh}_{3}\right)_{4}$, toluene, $85{ }^{\circ} \mathrm{C}, 48 \mathrm{~h}, 58.3 \%$ (P7) yield. (iv) $\mathrm{CF}_{3} \mathrm{COOH}, \mathrm{CH}_{2} \mathrm{Cl}_{2}$, R.T., 96.5\% (P8a) yield. (v) annealed at $200{ }^{\circ} \mathrm{C}$.

Scheme 9: The synthesis of PDFTBT-Th4 via DAP and Stille polymerization methods. The synthesis of PDFTBT-Th4 via DAP includes the application of small molecule direct arylation to generate one of the monomers. This enables the synthesis of DAP PDFTBT-Th 4 in only 3 steps while the synthesis of Stille PDFTBT-Th 4 requires 4 steps. Conditions are as follows: (i) $\mathrm{Pd}_{2}(\mathrm{dba})_{3},\left((\mathrm{o}-\mathrm{MeOPh})_{3} \mathrm{P}, \mathrm{K}_{2} \mathrm{CO}_{3}\right.$, PivOH, o-xylene, $61.0 \%$ yield for (compound 22); (ii) NBS, THF, $89 \%$ yield for (compound 23); (iii) n-BuLi, THF, $-78^{\circ} \mathrm{C}, \mathrm{SnMe}_{3} \mathrm{Cl}$; (iv) DAP Protocol (See Table 5.1); (v) $\mathrm{P}(\mathrm{o}-\mathrm{tol})_{3}, \mathrm{Pd}_{2}(\mathrm{dba})_{3}$, o-xylene, $56 \%$ yield.

Scheme 10: Synthetic route to PDFBT-DTP polymer via DAP. (i) $\mathrm{NaBO}_{3} \cdot \mathrm{H}_{2} \mathrm{O}$, $\mathrm{CHCl}_{3}$, TFA, R.T., 27\% yiled (compound 24). (ii) $\mathrm{BrMgC}_{10} \mathrm{H}_{21}$, THF, $<-20{ }^{\circ} \mathrm{C}, 86 \%$ yield (compound 25). (iii) p-TSA. $\mathrm{H}_{2} \mathrm{O}$, toluene, $110{ }^{\circ} \mathrm{C}, 95 \%$ yield (compound 26). (iv) DAP Protocol (See Table 6.1). 195

Scheme 11: Synthesis of 8,11-dibromodithieno[3,2-a:2',3'-c]phenazine, DBDTP. (i) $\mathrm{NaBH}_{4}$, EtOH, 6 h, R.T. 76\% yiled (compound 27). (ii) AcOH, EtOH, reflux, 48 h, and $86 \%$ yield (compound $\mathbf{2 8}$ ). .228

Scheme 12: Synthesis of DTP-CMP via one-step direct arylation polymerization of DBDTP as a single monomer. (i) $\mathrm{Pd}_{2}(\mathrm{dba})_{3},(\mathrm{o}-\mathrm{MeOPh})_{3} \mathrm{P}, \mathrm{K}_{2} \mathrm{CO}_{3}$, $\mathrm{PiVOH}$, o-xylene (see DAP protocol in section 7.2.2). 229

Scheme 13: Examples of proposed monomers and polymers for organic electronics applications. 251

Scheme 14: Examples of proposed monomers for the synthesis of D-A conjugated polymers and conjugated microporous polymers (CMPs) via DAP. .253 


\section{List of Tables}

Table 2.1. Molecular weights and thermal properties of D-A polymers................82

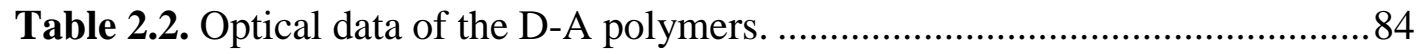

Table 2.3. Electrochemical properties of D-A polymers..................................... 86

Table 2.4. Photovoltaic performance of $\mathrm{D}-\mathrm{A}$ polymers with $\mathrm{PC}_{71} \mathrm{BM}$ under illumination of AM 1.5G, $940 \mathrm{~W} / \mathrm{m}^{2}$ (Active layer (spin seeped at $1000 \mathrm{rpm}$ ): D-A Polymer/PC ${ }_{71} \mathrm{BM}(1 / 2$ by weight) with $3 \%$ of $\mathrm{DIO} ; 10-12$ devices were evaluated

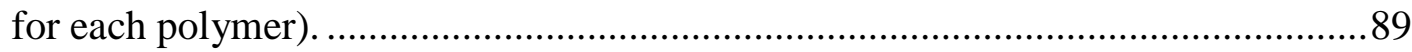

Table 2.5. Photovoltaic performance of $\mathrm{D}-\mathrm{A}$ polymers with $\mathrm{PC}_{71} \mathrm{BM}$ (1:2 by weight) and $3 \%$ of DIO additive as active layers (spin seeped at $500 \mathrm{rpm}$ ) under the illumination of $\mathrm{AM} 1.5 \mathrm{G}, 940 \mathrm{~W} / \mathrm{m}^{2}$. $(10$ - 12 devices were evaluated for each polymer).

Table 2.6. Performance characteristics of OFET devices with D-A polymer semiconductor on an OTS-8-treatd substrate fabricated and measured under ambient conditions.

Table 2.7. Performance characteristics of OFET devices with D-A polymer semiconductor on an OTS-18-treatd substrate fabricated and measured. .94

Table 3.1. Optical properties of P5 and P6 polymers. 119

Table 3.2. Electrochemical properties of $\mathbf{P 5}$ and $\mathbf{P 6}$ polymers. 121

Table 3.3. Performance characteristics of OFET devices with P5 and P6 polymer semiconductor on an OTS-18-treated substrate fabricated and measured under a nitrogen atmosphere 126

Table 4.1. Molecular weights and thermal properties of P7 and P8a copolymers.

Table 4.2. Optical data of the polymers, P7 and P8a. 148

Table 4.3. Electrochemical properties of polymers. 151 
Table 4.4. Performance characteristics of OFET devices for $\mathbf{P 7}$ polymer semiconductor on an OTS-18-treatd substrate fabricated and measured ambient conditions. 152

Table 5.1. DAP reaction optimization entries for the synthesis of the PDFBT-Th4 polymer. 171

Table 5.2. Optical data of PDFBT-Th4 4 polymers. 180

Table 5.3. Electrochemical properties of PDFBT-Th4 polymers. 181

Table 5.4. Performance characteristics of OFET devices for PDFBT-Th 4 polymers (E3a, E5, and Stille-B1 polymer) semiconductor on an OTS-18-treatd substrate fabricated and measured ambient conditions. 184

Table 5.5. Photovoltaic performance of PDFBT-Th4 polymers (E3a-DAP and Stille-B2) 189

Table 6.1. DAP optimization entries for synthesis the PDFBT-DTP polymer....205

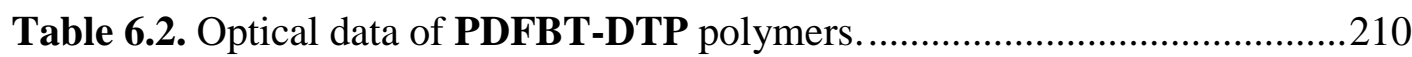

Table 6.3. Electrochemical properties of PDFBT-DTP polymers. ......................212

Table 6.4. Performance characteristics of OFET devices for PDFBT-DTP polymer (E2-DTP) semiconductor on an OTS-18-treatd substrate fabricated and measured ambient conditions. 215

Table 7.1. Peak assignments for an FT-IR spectrum of DTP-CMP .231

Table 7.2. Electrochemical properties of DTP-CMP polymers. .236

Table 7.3. Porosity properties of DTP-CMP polymers. .239 


\section{ABSTRACT}

Conjugated polymer semiconductors have gained a tremendous development in terms of their design and synthesis diversity for applications in different optoelectronic devices such as organic field-effect transistors (OFETs), organic photovoltaics (OPVs), and organic light emitting diodes (OLEDs). Recent studies demonstrated that the design strategy of combining an electron donor (D) and acceptor (A) moieties into the polymer backbone results in high-performance organic semiconductors, as it gives a means to tune physicoelectrochemical properties of the resulting conjugated polymer. D-A conjugated polymers are mainly synthesized via traditional coupling techniques (such as Stille and Suzuki couplings) and also by using a highly anticipated greener and economical method, direct $\mathrm{C}-\mathrm{H}$ arylation polymerization (DAP).

In this research work, new electron acceptor moieties, 2,1,3-dithienobenzochalcogenodiazole (2,1,3-dithienobenzo-thiadiazole and 2,1,3-dithienobenzooxadiazole) and $\gamma$-lactone-Pechmann dye were synthesized and incorporated into the construction of D-A conjugated polymers by using traditional coupling methods (such as Stille and Suzuki couplings). Detailed systematic investigations were carried out to study the effects of chalcogen atoms and different electron donor moieties on the optical and electrochemical properties, as well as on OPV and OFET performance of their corresponding D-A conjugated polymers. Thermogravimetric analysis 
results show the good thermal stability of all polymers with decomposition temperature $\left(T_{\mathrm{d}}\right)$ at $5 \%$ weight loss above $300{ }^{\circ} \mathrm{C}$. The UV-vis-NIR spectra of $\gamma$ lactone-Pechmann dye based D-A polymers shows a broad absorption band extended to $1000 \mathrm{~nm}$ and narrow band gaps with deep LUMO energy levels. D-A polymers containing 2,1,3-benzooxadiazole moiety shows deeper LUMO level as compared to their 2,1,3-benzothiadiazole-containing analogs, whereas polymers containing weak electron donor moiety (alkylidenefluorene) exhibited deeper HOMO levels than polymers with stronger electron donors. Photovoltaic power conversion efficiency of over $2 \%$, hole field-effect mobility of $2.6 \times 10^{-2} \mathrm{~cm}^{2} \mathrm{~V}^{-1} \mathrm{~s}^{-1}$ and on/off ratio of over $10^{5}$ were obtained.

Another D-A conjugated polymers based on tert-butoxycarbonyl (t-Boc) substituted isoindigo as electron acceptor moiety and dithiophene-benzodithiophene derivative as electron donor moieties designed and synthesized via Stille polymerization method. The elimination of bulky t-Boc side groups resulted in the emergence of $\mathrm{N}-\mathrm{H} \cdots \mathrm{O}=\mathrm{C}$ hydrogen bonding interactions. It results in an increased field-effect mobility of the polymer after chemical or thermal treatment, which may arise from the enhanced planarity and intermolecular ordering of the isoindigo units.

Direct $\mathrm{C}-\mathrm{H}$ arylation polymerization (DAP) as a facile, cheap, and atom economy synthesis protocol was applied for the synthesis of an alternating 5,6difluoro-2,1,3-benzothiadiazole based low bandgap D-A conjugated polymers. The DAP reaction conditions were optimized, and a polymer with number-average 
molecular weight $\left(M_{\mathrm{n}}\right)$ of $27 \mathrm{kDa}$ was obtained under the optimal catalytic condition of $\mathrm{Pd}(\mathrm{OAc})_{2} /(\mathrm{o}-\mathrm{MeOPh})_{3} \mathrm{P} / \mathrm{PivOH} / \mathrm{K}_{2} \mathrm{CO}_{3}$ in o-xylene. UV-vis absorption spectra of the polymers indicated strong interchain aggregation in films. While the $\mathrm{C}-\mathrm{H}$ selectivity and the alternating polymer structure of the polymers synthesized via DAP are comparable to those of the same type polymers synthesized via Stille coupling. The batch of D-A polymer synthesized via optimal DAP, despite its lower $M_{\mathrm{n}}$, showed higher hole mobility of $7.5 \times 10^{-3} \mathrm{~cm}^{2} \mathrm{~V}^{1} \mathrm{~s}^{1}$ in OFETs and larger power conversion efficiency of $4 \%$ in OPVs.

The versatility of DAP for synthesizing other types of conjugated polymer networks was investigated by applying it to the synthesis of conjugated microporous polymers (CMPs) based on a single monomer unit, 8,11-dibromodithieno [3,2$\left.\mathrm{a}: 2^{\prime}, 3^{\prime}-\mathrm{c}\right]$ phenazine. The resulting polymers exhibit hierarchical porous structures and a narrow bandgap of $1.5 \mathrm{eV}$.

The findings in this research work could help to the future developments of conjugated polymers, in their design, synthesis, and application for organic electronics devices (OFETs and OPVs). 


\section{CHAPTER 1}

\section{General Introduction}

The increasing demand for a renewable energy source and the need for cheap and flexible electronic devices have been a driving force for the development of organic semiconductors. The main components of these electronic devices are a semiconductor, which could be inorganic (like silicon, germanium) or organic (like conjugated polymers) semiconductors. The current state-of-the-art of semiconducting building materials for electronics devices application is mainly inorganic semiconductors for their ability to provide high performance and stability. However, their requirement of high processing technology, large cost and the lack of flexibility have been the driving force for the emergence of organic (plastic) semiconductors. ${ }^{1}$ The ability of organic semiconductors to combine the electrical properties of inorganic semiconductors and the properties typical to organic compounds such as solution-processability, mechanical flexibility, versatility of chemical synthesis and possibility of structural modification to tailor their physicoelectrochemical properties makes them an attractive option for electronic device applications. ${ }^{2}$ Therefore, organic semiconductors could enable a potentially flexible, cheap, and large area printed integrated circuit devices like organic field 
effect transistors (OFETs), organic photovoltaics (OPVs), and organic light emitting diodes (OLEDs). ${ }^{1}$ Thus, along with this line, a tremendous progress has been achieved in the development of organic electronic devices so that today it become a totally new field of the research area. The fundamental concepts and terminologies used in conjugated polymers (from synthesis to applications) in the organic electronics devices (like OPVs and OFETs) are described in this introductory Chapter. The first section describes the basics of conjugated polymers and their classifications ranging from linear (one-dimensional, 1D) to conjugated microporous polymers (CMPs). The next sections, illustrates the common architectures, operational principles, characteristics of organic photovoltaic and field effect transistor technologies. In addition, strategies for molecular design principles and common synthetic routes of conjugated polymers for OPVs and OFETs applications are presented. In the final section, a brief literature review on the development of conjugated polymers and scope of the thesis are explained.

\subsection{Conjugated Polymers}

Polymers are large molecules (macromolecules) composed of many repeating subunits has long been used as an insulator and commonly utilized in covering the electric appliance to avoid a direct contact of a human being with electricity. Therefore, it used to be an irrelevant idea to consider plastics could conduct electricity until the recent discoveries of conductive polymers. The polymers used as an insulator are mainly composed of saturated $\mathrm{sp}^{3}$-carbon atoms where the valence 
electrons are localized between the two neighboring $\mathrm{sp}^{3}$-carbon atoms connected by a single bond in the polymer backbone (which form a valence band, see Figure 1.1). The absence of electron delocalization, which is key to conductivity due to the presence of charge carrier mobility and excitation of an electron to the anti-bonding energy levels $\left(\sigma^{*}\right.$-levels) of the polymer. Therefore, these type of polymeric materials is of no interest with respect to electrical conduction as the generation of charge carrier could lead to the break-up (chain scission) of the polymer chain and consequently charge carriers become immobile.

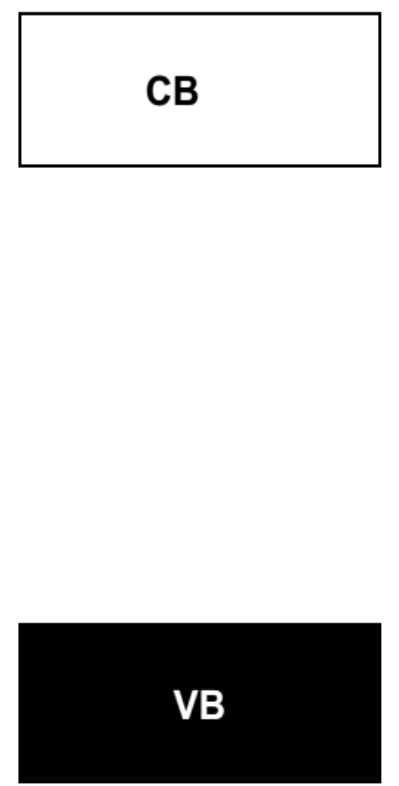

(A) Insulator
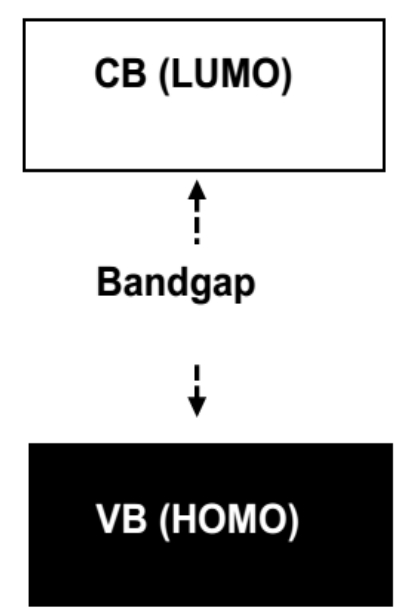

(B) Semiconductor

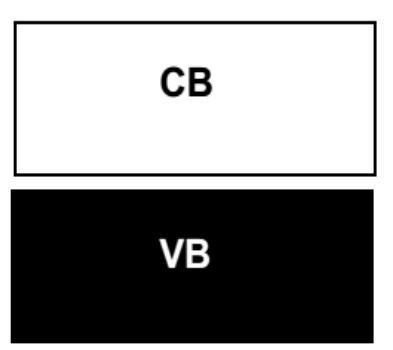

(C) Conductor

\section{$\mathrm{CB}=$ Conduction band, $\mathrm{VB}=$ Valence band}

Figure 1.1. A schematic representation of energy gap in (a) insulator (b) semiconductor (c) conductor. 
Conjugated polymers are one type of polymers having conjugated system and displays conductivity unlike another common type of polymers. After the discovery of highly conductive doped polyacetylene (PA) by Heeger, MacDiarmid, and Shirakawa in 1977 (they won Noble prize in chemistry) had propelled a huge research interest around the world to find a highly conductive conjugated polymer for different electronics device applications. ${ }^{3}$ In the structure of conjugated polymers, the mutual overlap between parallel p-orbitals in the adjacent $\mathrm{sp}^{2}$-carbon atoms along their backbone resulted in the formation of $\pi$-bond, thereby the delocalization of $\pi$ electrons along the entire conjugation path is enabled (see Figure 1.2). The delocalized $\pi$-electrons along the conjugated backbone of the polymer resulted in the formation of its intrinsic semiconductors behavior, which are combined to the desirable processing characteristics inherent to polymers with the sought-after electrical, electro-optic and nonlinear properties of semiconductors. ${ }^{4,5}$ Although, semiconducting behavior of conjugated polymers could be easily understood from their bandgap, the other requirements required for conjugated polymers to be considered as a semiconductors are like, the $\sigma$-bonds between the neighboring $\mathrm{sp}^{2}$ carbon atoms in the chain should be stronger than the $\pi$-bonds, thus which could hold the molecules intact even in their excited state. The other and most important requirement are that the neighboring parallel p-orbitals should overlap and thus form a three-dimensional $\pi$-band across the conjugated polymer backbone. ${ }^{6,7}$ Therefore, the charge carriers (electron and hole) could move either by hopping through the 
polymer backbone or tunneling between the neighboring polymer chains. The delocalization of $\pi$-electron across the conjugated polymer backbone resulted in the formation of field bonding state ( $\pi$-band) and the empty anti-bonding state ( $\pi^{*}$-band) (see Figure 1.1 and 1.2). Thus, the creation of energy gap between the $\pi$-band (highest occupied molecular orbital, HOMO) and $\pi^{*}$-band (lowest unoccupied molecular orbital, LUMO) determines most of the characteristics of the conjugated polymers. The bandgap of most conjugated polymers lies in the range between 1.5 to $3.0 \mathrm{eV}$ makes them ideal to be considered as a semiconductor.
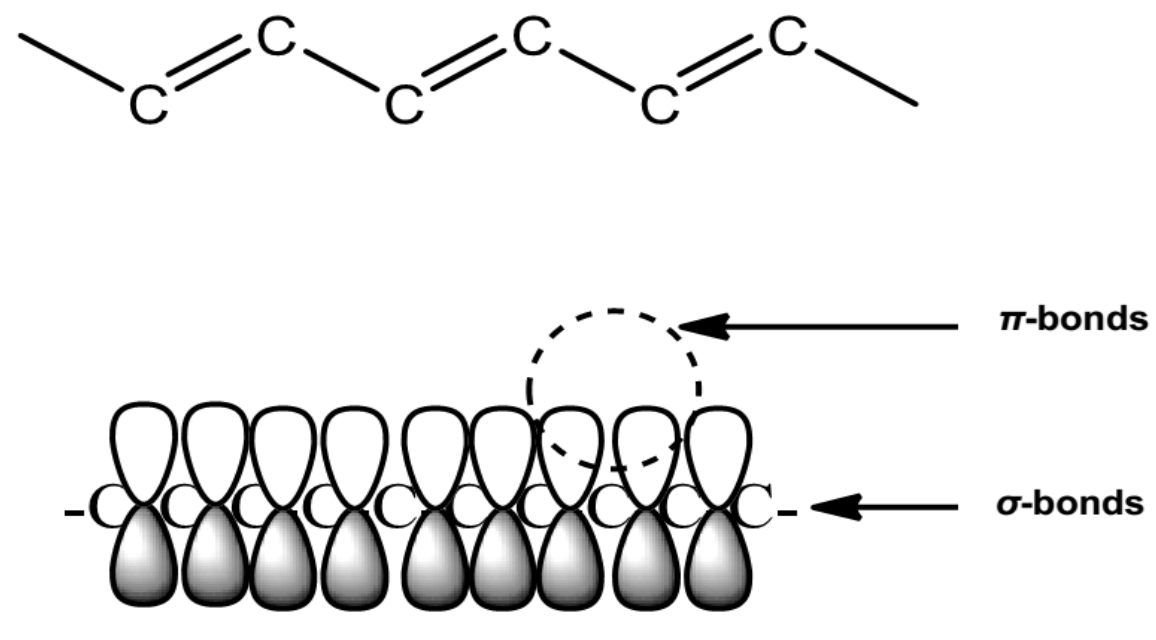

Figure 1.2. The molecular structure of polyacetylene $\left(\mathrm{C}_{2} \mathrm{H}_{2}\right)_{\mathrm{n}}$.

Conjugated polymers could be categorized according to the number of chain extension in the direction of the $\pi$-conjugation path. Most polymeric systems usually possess conventional linear, one-dimensional (1D) structures with continuous $\pi$ conjugations along the polymer backbone. Extending the $\pi$-conjugation in the lateral 
directions of the polymer backbone could lead to the formation of different supramolecular structures in the solid state and multidimensional charge transport. One-dimensional (1D)-linear conjugated polymers are mainly synthesized and used as an active layer in organic electronics device applications. For instance, the most commonly synthesized 1D-linear conjugated polymers are a homopolymer (P3HT, poly(3-hexylthiophene-2,5-diyl)), donor-acceptor (D-A) copolymer, and block copolymers. On the other hand, 1D-linear conjugated polymers could be extended to higher dimensionalities and sophisticated topologies to provide dendrimers, hyperbranched polymers, and conjugated networks. Among these networks, conjugated microporous polymers (CMPs) have attracted a great deal of attention in areas ranging from electronics to separation, because CMPs combines permanent microporosity with extended $\pi$-conjugation.

Therefore, this thesis comprised of synthesis, characterization, and applications (OPVs and OFETs) of 1D-linera conjugated polymers (from Chapter 2 to 6), which were synthesized by using a conventional Stille and Suzuki polymerization methods (polymers from Chapter 2 to 4) and also using a newly emerged greener and economical synthesis method, direct arylation polymerization (DAP) (polymers in Chapter 5 and 6). This DAP coupling method was extended to the synthesis of CMPs (in Chapter 7). The final Chapter presents the brief conclusion and suggestions of possible future works based on findings of this thesis. 


\subsection{Organic Photovoltaic Devices (OPVs)}

\subsubsection{Introduction}

In 1839, Becquerel has discovered a photocurrent could be generated when $\mathrm{AgBr}$ or $\mathrm{AgCl}$ covered platinum electrodes illuminated in aqueous solution. ${ }^{8}$ Fortyfour years later, Selenium wafer-based devices were developed by Fritts to give a power conversion efficiency (PCE) of $\sim 1 \% .{ }^{9}$ Then, in 1954 silicon based inorganic

solar cell was developed for the first time with PCE of $6 \%$ at Bell Laboratories. ${ }^{10}$ Over the years the PCE of crystalline silicon solar cells has reached $27.6 \% .^{11}$ However, the high cost of processing and difficulties of getting good quality siliconbased semiconductors devices the need for new and inexpensive technologies for processing and fabricating silicon-based semiconductors for photovoltaics applications. In this aspect, organic semiconductors and also called plastic semiconductors are the best alternatives due to their attractive advantages over their inorganic semiconductors counterpart. For instance, organic semiconductors can be easily prepared via various synthetic methodologies and it provides a means to tailor their physicoelectrochemical properties via changing the functionalities of their conjugated backbones. In addition to this, most organic semiconductors can be solution processable and therefore, it can be deposited on different substrates to give flexible, transparent and overall cheap photovoltaic devices. 


\subsubsection{Structure of OPVs}

A simple and conventional structure of the solar cell is shown in Figure 1.3. The difference between OPVs and crystalline silicon photovoltaics mainly lies on the materials used as a photoactive layer. In OPVs, conjugated polymers donor blended with PCBM acceptor in bulk heterojunction (BHJ) configuration are mainly used as photoactive materials to absorb sunlight, while in crystalline silicon photovoltaics, silicon wafers are used as the photoactive layer. Besides this, usually metals having low work function (like $\mathrm{Al}, \mathrm{Mg}$, or $\mathrm{Ca}$ ) are used as the cathode electrode. High work function metals (like thin doped indium oxide, ITO) are used as an anode electrode, and PEDOT: PSS dispersed on ITO surface to increase its conductivity. Finally, all components of OPV, like electrodes and the active layer are deposited on the surface of the transparent glass substrate in sequence as shown in Figure 1.3.

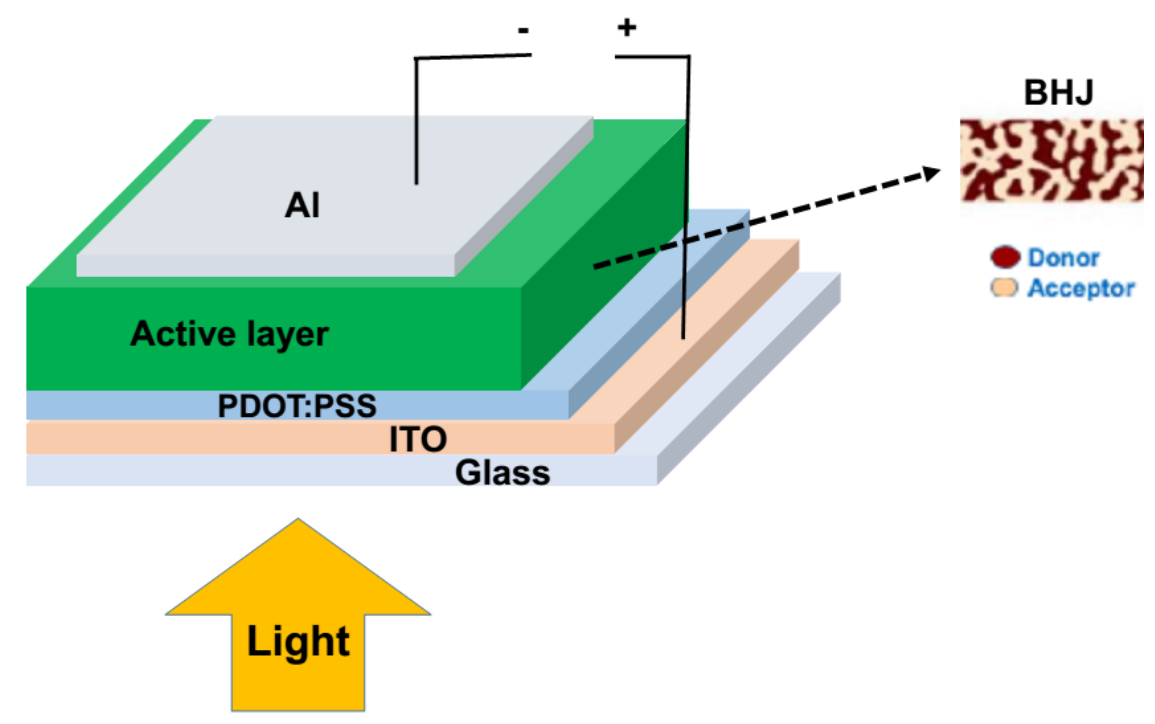

Figure 1.3. The architecture of the the conventional organic photovoltaic device. 


\subsubsection{Operation Principles of OPVs}

The first step in the photoconversion process is absorption of light by the photoactive layer of organic photovoltaic devices, which is mainly to excite an electron from $\pi$-bonding orbital (HOMO) to a $\pi$-antibonding orbital (LUMO) ( $\pi$ to $\left.\pi^{*}\right)$ of the polymer donor material, this process is called exciton formation (coulombically bound state of an electron and electron hole pairs). For exciton formation normally the energy of photon should be higher than the bandgap of donor polymer material in the active layer. The next step is exciton diffusion to the donoracceptor interface and then followed by dissociation via an electron transfer process from the LUMO of donor polymer to LUMO of acceptor but holes remain in the HOMO of donor polymer. The Strong electric field is required for excitons dissociation into free charge carriers, which mainly comes from minimum energy level offset (>0.3 eV) between LUMOs. Smaller or larger energy level offset will reduce the overall PCE of the organic solar cell through recombination or decreased $\mathrm{V}_{\mathrm{oc}}$ values, respectively. Therefore, an optimum energy level offset is required for efficient charge separation which could lead to high PCE. Generally, organic semiconductors have larger binding energy than their inorganic counterparts so that excitons diffuse shorter distance ca.10 nm. To obtain ca. $10 \mathrm{~nm}$ diffusion length, it requires thin active layer within $10 \mathrm{~nm}$ thickness. Otherwise, excitons created far

away from the donor-acceptor interface decay to the ground state without dissociation. Finally, these free charge carriers migrate to their corresponding 
electrodes under the force of built-in potential to produce photocurrent and photovoltage. In short, the overall photoconversion process is depicted in Figure 1.4.

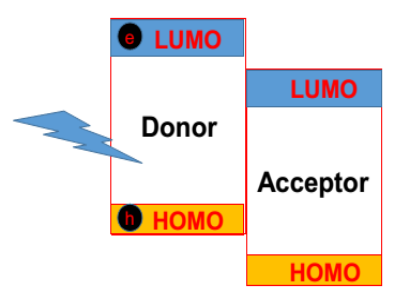

(1) Photoexcitation and exciton formation

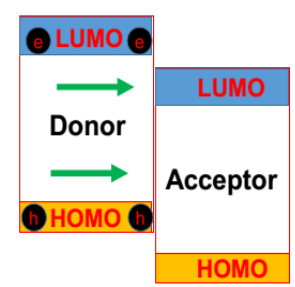

(2) Exciton diffusion

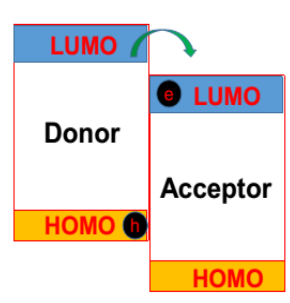

(3) Exciton dissociation (charge separation)

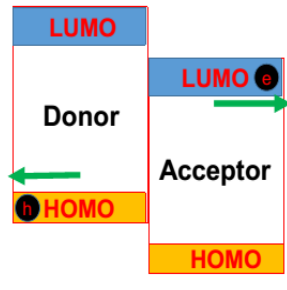

(4) Charge transport and collection

Figure 1.4. The photoconversion mechanism of an OPV devices.

\subsubsection{Device Architectures of OPVs}

OPVs can be divided into three categories such as bilayer heterojunction, bulk heterojunction $(\mathrm{BHJ})$, and inverted heterojunction devices based on the respective arrangement of active layers, electrodes, and interface layers.

\subsubsection{Bilayer heterojunction OPV devices}

As shown in Figure 1.5, sequentially stacked organic semiconductors p-type and n-type semiconductors, p-type is electron rich while n-type is electron deficient; on top of each other gives a bilayer heterojunction type of OPVs. This type of device architecture was realized for different combinations of organic semiconductors. ${ }^{12-20}$ However, obtaining a very thin active layer with thickness up to $10-20 \mathrm{~nm}$ is a difficult task, because the created excitons have a very short diffusion length in the range of 10-20 $\mathrm{nm}$ to the donor-acceptor interface. Thus, excitons created out of this diffusion length will decay and thus decrease the quantum efficiency. ${ }^{21}$ Therefore, a 
bilayer heterojunction OPVs is greatly dependent on the thickness of the active layer, but the requirement of very thin active layer limits the amount of absorbed photon and consequently limits the overall efficiency. ${ }^{22-24}$ This problem anticipates scientists to search a better architecture of active layer in OPVs, for instance, bulk heterojunction (BHJ).

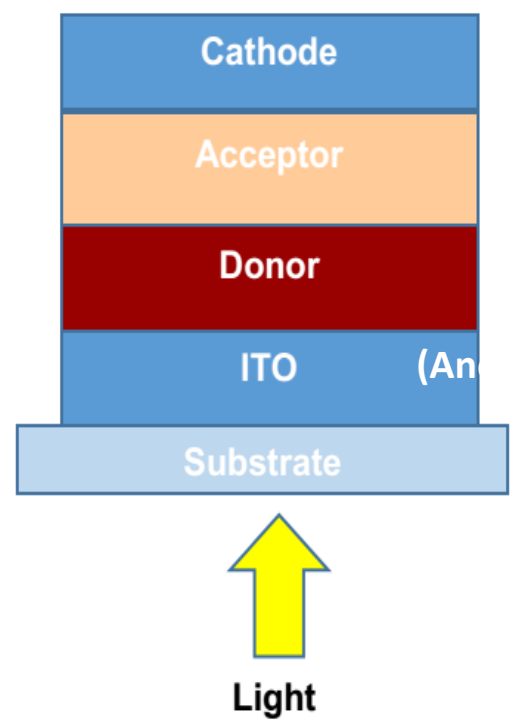

Figure 1.5. Schematic representation of bilayer heterojunction devices.

\subsubsection{Bulk heterojunction (BHJ) OPV devices}

The concept of bulk heterojunction active layer was introduced in order to improve the PCE of organic solar cell, where donor and acceptor components of the active layer were blended in a bulk volume to create an interpenetrating phase (see Figure 1.3). Therefore, it helps to increase the interfacial area and also could avoid the thickness (excitons diffusion length) related problem as in the bilayer heterojunction devices. ${ }^{25}$ In bilayer devices, the donor and acceptor components of 
the active layer selectively contact with the anode and cathode electrodes, respectively. Whereas in $\mathrm{BHJ}$ devices an intermixing of the active layer components result in the creation of no symmetry breaking point of the electric field, that is, the created charge carriers (holes and electrons) have no preferred net direction to move. Therefore, a symmetry breaking should be done by using electrodes having different work function. In addition, the efficiency of BHJ devices could be increased via controlling an interpenetrating nanoscale, bicontinuous morphologies of the active layer through co-deposition of donor-acceptor blends of active layer. ${ }^{26-30}$

\subsubsection{Inverted bulk heterojunction OPV devices}

In the conventional BHJ-OPV devices (see Figure 1.3), the electrons are collected by the low work function metals, such as $\mathrm{Al}$, or $\mathrm{Ca}$ (cathode electrode) and holes are collected by ITO (anode electrode) coated with a hole conducting layer (PEDOT: PSS). However, this device architecture usually suffer from stability issue due to the oxidation of cathode electrode, fast degradation of PEDOT: PSS layer due to its acidic nature, and the vertical phase separation between polymer donor (mainly concentrated on the top) and fullerene acceptor (mainly concentrated on the bottom) during the film formation of their BHJ. ${ }^{31-34}$ Thus, the vertical separation is opposite to the ideally expected flow of charge carriers to their respective electrodes. All these drawbacks could be avoided by inverting the charge collection process via using a high work function metal like ( $\mathrm{Ag}$ or $\mathrm{Au})$ as a top electrode to collect holes (anode) 
and ITO as a bottom electrode to collect electrons (cathode). The ITO work function (4.5 - $4.7 \mathrm{eV}$, see Figure 1.6 (b)) lies in between of the HOMO-LUMO energy levels of most conjugated polymer donor materials, thus which could be used either to collect electron (cathode, in inverted BHJ-OPV) or hole (anode, in conventional BHJ-OPV). Therefore, this versatility of ITO could help in making a new configuration of OPVs called inverted-BHJ OPVs (see Figure 1.6 (a)). In this type of configuration, usually ITO is covered with a hole blocking layer material (like $\mathrm{ZnO}, \mathrm{TiO}_{2}$, or $\operatorname{In}_{2} \mathrm{~S}_{3}$ ) and the anode electrodes are deposited on the top of the electron blocking layer metal oxides, (like $\mathrm{MoO}_{3}$, or $\mathrm{V}_{2} \mathrm{O}_{5}$ ). Thus, it improves the overall stability and consequently PCE of the resulting OPVs. ${ }^{35-42}$ As describes earlier, Inverted-BHJ OPVs have good advantages over the conventional-BHJ OPVs. Therefore, in this thesis inverted-BHJ OPVs configuration is adopted to fabricate and evaluate the performance of all the synthesized D-A conjugated semiconducting polymers. 

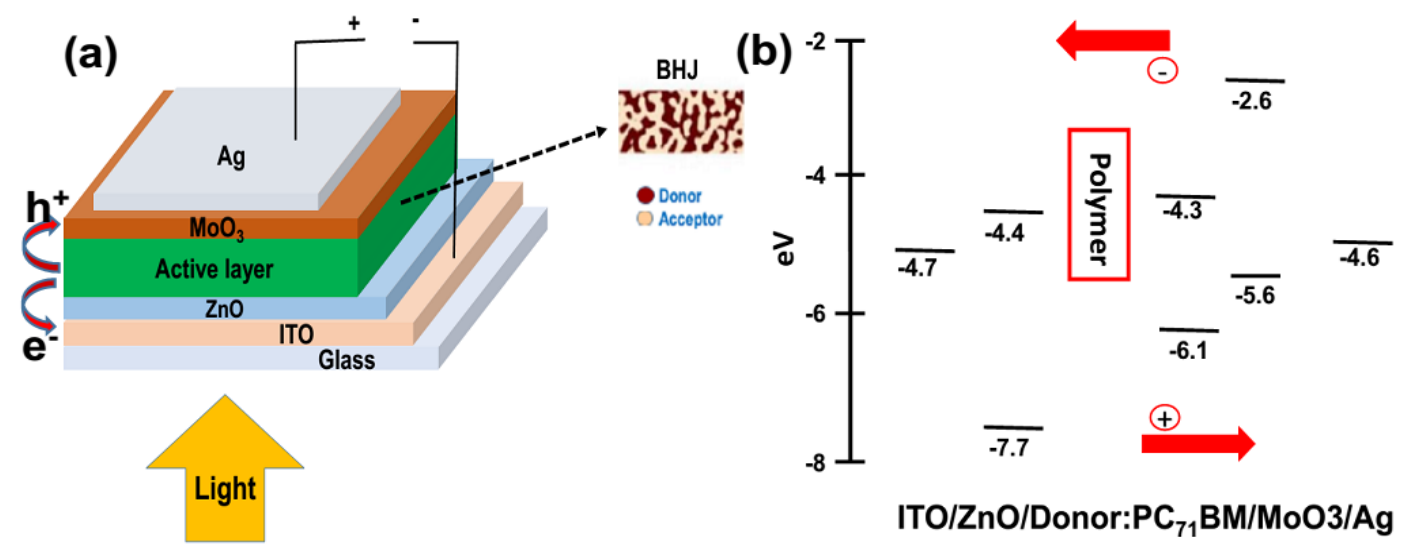

Figure 1.6. Schematic representation of inverted-BHJ OPV (a) and an energy level diagram of Inverted-BHJ OPV (b).

\subsubsection{Design strategy towards high-efficiency OPVs materials}

A careful optimization of each photoconversion steps (see Figure 1.4) is required to get high PCE of BHJ-OPVs. Scharber contour plot shows that theoretically calculated maximum PCE of a polymer donor material with bandgap of $1.5 \mathrm{eV}, \mathrm{FF}$ of $65 \%$, and incident photon to current efficiency (IPCE) of $60 \%$ could reach above $13 \%{ }^{43} \mathrm{~A}$ better understanding of the working mechanism of photoconversion process and the factors which limit the efficiency below its theoretically expected value is quite important to better understand the design and synthesis of a new donor conjugated polymers for OPVs. Some of the main factors that limit the PCE are like lack of broad absorption range of the solar spectrum, low carrier mobility, low exciton diffusion length, and the energy level mismatch between PCBM acceptor and polymer donor. ${ }^{44}$ 
Power conversion efficiency (PCE): is an important parameter which describes the performance of a given OPVs. As shown in Figure 1.7, it is described as a function of parameters like open circuit voltage $\left(\mathrm{V}_{\mathrm{oc}}\right)$, short-circuit current density $\left(\mathrm{J}_{\mathrm{sc}}\right)$, and fill factor $(\mathrm{FF})$. The value for all of these parameters can be extracted from current-voltage (I-V) curves under the 1 sun condition $\left(100 \mathrm{~mW} / \mathrm{cm}^{2}\right.$, simulated AM1.5 solar illumination) which is referred as $\mathrm{P}_{\text {in }}$.

$$
\text { PCE }=\frac{J_{\mathrm{sc}} * V_{\text {oc }} * F F}{p_{\text {in }}}
$$

Open Circuit Voltage $\left(\mathbf{V}_{\text {oc }}\right)$ : is correlated with the difference in energy between the LUMO of PCBM acceptor and HOMO of polymer donor. Theoretically; the high value of $\mathrm{V}_{\mathrm{oc}}$ could be obtained by lowering the HOMO level of the donor. However, it couldn't be lowered below $0.3 \mathrm{eV}$ energy level offset between the LUMOs of PCBM acceptor and polymer donor, as it negatively affects the efficient charge separation process. On the other hand, lowering HOMO while keeping constant LUMO will increase bandgap of a polymer donor, thus, consequently decreases the maximum possible absorption of solar energy and thus decreases the maximum obtainable value of $\mathrm{J}_{\mathrm{sc}}$.

Short Circuit Current $\left(\mathbf{J}_{\mathrm{sc}}\right)$ : is directly related to a number of excitons created during illumination active layer with solar light. Ideally, the active layer should absorb a maximum of solar light to increase the number of excitons creation. The major responsibility to absorb solar energy lies on the polymer donor part of the 
active layer, as the maximum number of photons lies above $600 \mathrm{~nm}$ of the solar spectrum, and thus PCBM has poor absorption capacity in the longer wavelength region of the solar spectrum. Therefore, maximizing $\mathrm{J}_{\mathrm{sc}}$ of an OPV device could be achieved via designing a low bandgap polymer donor.

Fill Factor (FF): is the ratio between the product of voltage and current at maximum power point $\left(\mathrm{J}_{\mathrm{mp}}, \mathrm{V}_{\mathrm{mp}}\right)$ to $\mathrm{J}_{\mathrm{sc}}$, with $\mathrm{V}_{\mathrm{oc}}$. This ratio can be increased to unity via designing active layer to have high mobility, a high degree of crystallinity, better morphology. Generally, these parameters are hugely influenced by incorporating a fused ring monomers to the polymer backbone. Therefore, planner conformation and extended $\pi$-conjugation length of polymer donor materials could improve the fill factor.

$$
\mathbf{F F}=\frac{\operatorname{area} A}{\operatorname{area~} B}=\frac{J_{\mathrm{mp}} * V_{\mathrm{mp}}}{J_{\mathrm{sc}} * V_{\mathrm{oc}}}
$$

In summary, while designing a polymer donor material one should take into consideration on how to compromise the $\mathrm{J}_{\mathrm{sc}}, \mathrm{V}_{\mathrm{oc}}$ and $\mathrm{FF}$ values in order to maximize the PCE of an OPV device. Therefore, the copolymerization of electron rich (Donor) and electron deficient (Acceptor) moieties is one of the most widely used design strategy for the synthesis of conjugated polymers, as it give a means to tailor the physicoelectrochemical properties of the resulting D-A conjugated polymers and thus it helps to compromise the values of $\mathrm{J}_{\mathrm{sc}}, \mathrm{V}_{\mathrm{oc}}$, and FF. 


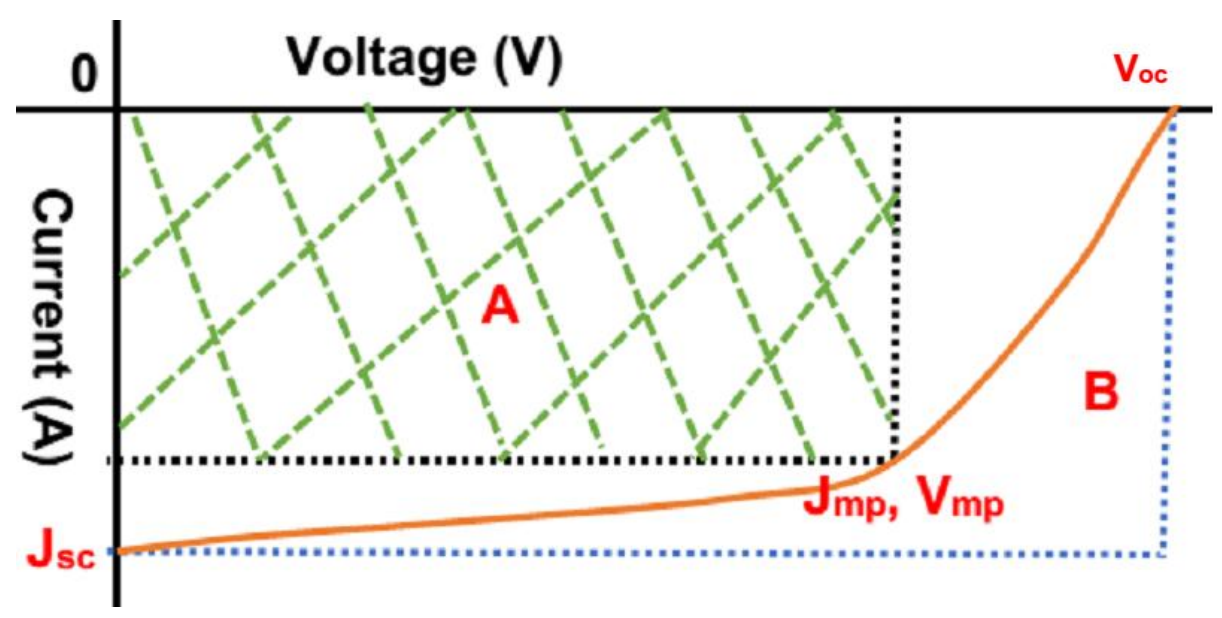

Figure 1.7. Illustration for the typical current-voltage (I-V) characteristics curve.

\subsection{Organic Field Effect Transistors (OFETs)}

\subsubsection{Introduction}

Since 1930 proposal of Lilienfeld on the principles field effect transistor (FET),${ }^{45}$ the development of FET as a major component of modern microelectronics devices has gained a tremendous interest and development. Field effect transistors are used to modulate the flow of current in the channel by applying an external bias (voltage), and thus it operates as an on/off switch and capacitor. In 1986, Koezuka and coworkers have developed organic field effect transistors (OFETs) based on conjugated polymer as a channel semiconductor with charge carrier mobility of $10^{-5}$ $\mathrm{cm}^{2} \cdot \mathrm{V}^{-1} \cdot \mathrm{s}^{-1} \cdot{ }^{46,47}$ Since then, large number of conjugated polymers based OFETs have been reported with high charge carrier mobility. ${ }^{48,49}$ 


\subsubsection{Structures of OFETs}

Figure 1.8 shows the four common types of OFET devices which usually consist of there-electrode (such as drain, source, and gate), an insulating dielectric layer, and a channel organic semiconducting material sandwiched between the drain and source electrodes. Usually, materials like a highly n-doped Si wafer are used as a gate electrode, $\mathrm{SiO}_{2}$ as the dielectric layer, and metals (like $\mathrm{Al}, \mathrm{Ag}$, or $\mathrm{Au}$ ) as source and drain electrodes are used in OFETs. Based on the respective arrangement of this three-electrode with a channel semiconducting layer, OFETs could be categorized into four type (see Figure 1.8). In top contact geometry, the source and drain electrodes are deposited on the top of the semiconducting layer (see Figure 1.8 (a \& c)). In both cases, the source and drain electrodes are deposited on the top of the semiconducting layer via vacuum thermal evaporation through a shadow mask. However, in bottom contact devices the semiconducting channel is deposited on the top of lithographically pre-patterned substrate (see Figure 1.8(d)) or on the dielectric layer (see Figure 1.8(b))..$^{50-52}$ The top gate device could help to encapsulate the semiconducting layer and then to prevent the degradation of the semiconducting channel upon exposure to air, subsequently which improves the overall stability of the device. ${ }^{53}$ 
(A) Bottom Gate / Top Contact

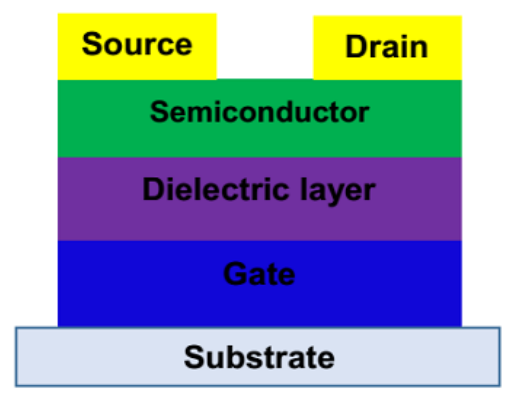

(B) Bottom Gate / Bottom Contact

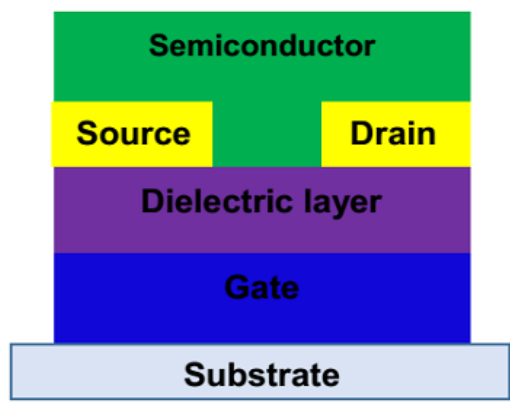

(C) Top Gate / Top Contact

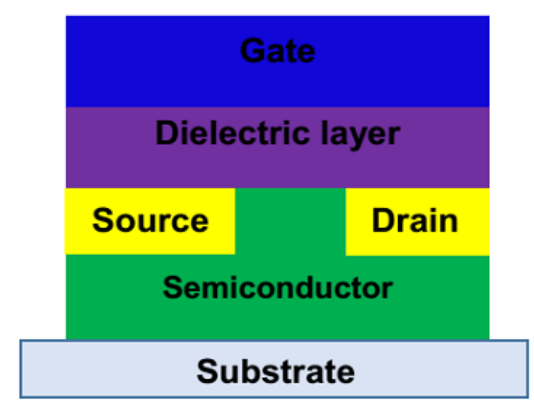

(D)Top Gate / Bottom Contact

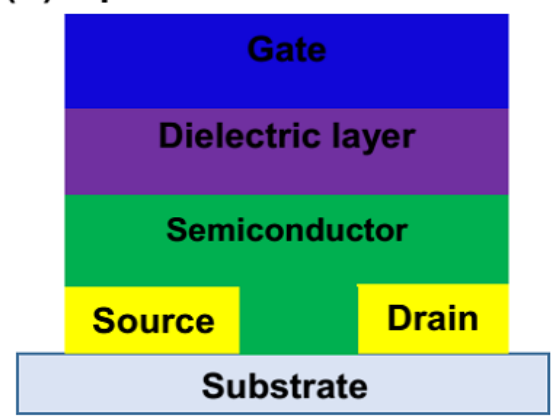

Figure 1.8. Four common types of OFETs structures top contact (a), (c) and bottom contact (b), (d) OFET devices.

\subsubsection{Operation Principles of OFETs}

Transistors are main components of modern electronic devices and they can be used to control the flow of current. As described earlier, they are composed of three electrodes; gate, source, and drain. Therefore, by applying a bias across gate-source $\left(\mathrm{V}_{\mathrm{gs}}\right)$ and source-drain $\left(\mathrm{V}_{\mathrm{ds}}\right)$ electrodes, it could be possible to control the flow of current between the drain and source electrodes. For instance, if the $V_{\mathrm{gs}}=0$, there should be no current flow between drain and source electrodes, in this case, the transistor is in its off state. However, if $\mathrm{V}_{\mathrm{gs}} \# 0$ (either negative or positive bias) and 
$\mathrm{V}_{\mathrm{ds}}=0$, then charges (either electron or hole) are accumulated at the interface between dielectric layer and semiconductor. In this case, the transistor serves as a capacitor (see Figure 1.9 (a)). The created strong electric field could cause a change in the energy levels (HOMO and LUMO) of the organic semiconductor. A semiconducting channel is created between the drain and source electrodes depending on the work function of the electrodes with respect to the energy level of organic semiconductor used. When $\mathrm{V}_{\mathrm{ds}} \# 0$, causes current to be driven through the device (see Figure 1.9 (b)). However, factors like the mismatch of energy between the organic semiconductor and electrodes (drain and sources) and also the presence of trap at the dielectric layer-organic semiconductor interface could affect the flow of current (mobility) between the channel semiconductor and electrodes.

\section{(A) Charges accumulated layer}

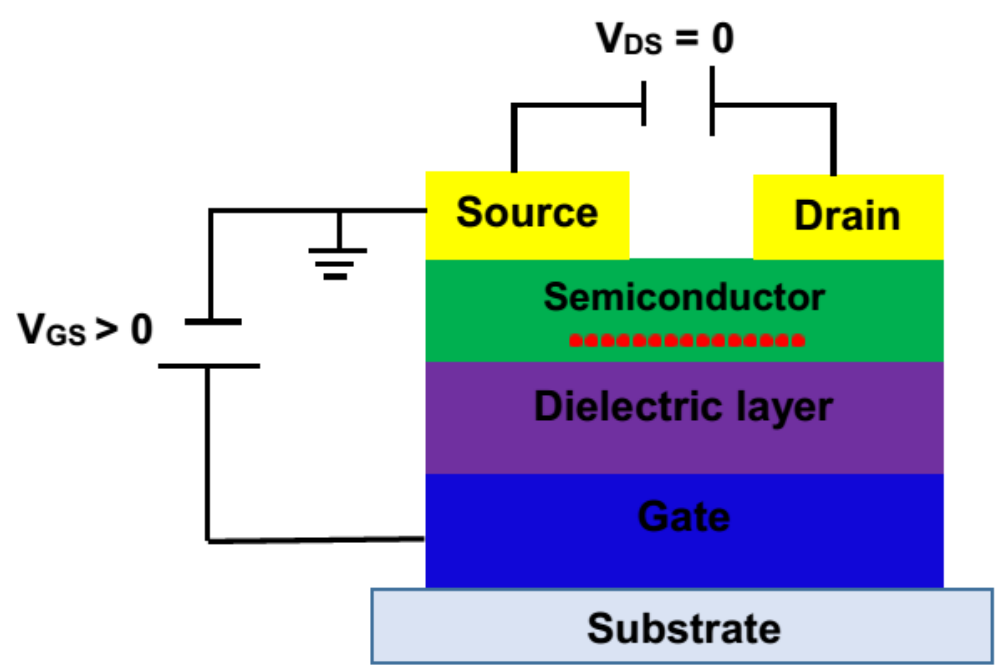


(B) Charge (hole or electron) flow

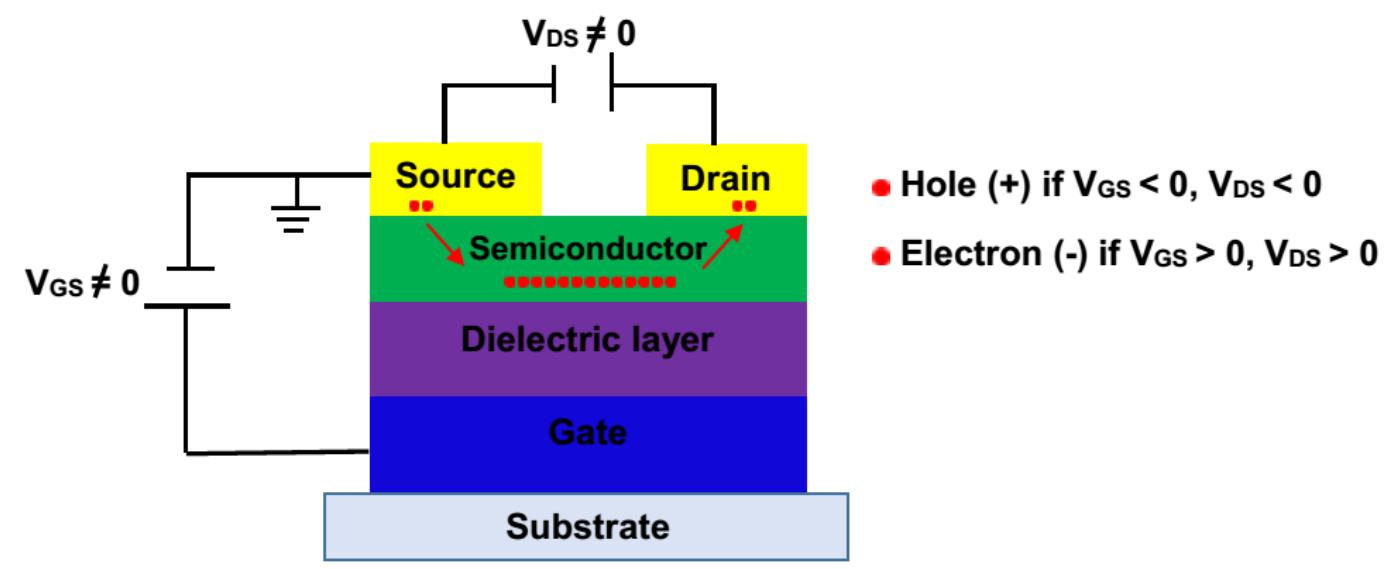

Figure 1.9. Schematic representation of OFETs operating principles (a) Charge carriers (holes (+) or electron (-)) forming accumulation layer. (b) Charge flow.

\subsubsection{Characteristics of OFETs}

The charge carrier mobility of an OFET device is an important parameter together with the current on/off $\left(\mathrm{I}_{\mathrm{on}} / \mathrm{I}_{\text {off }}\right)$ ratio, and threshold voltage. The charge mobility is defined as the average drift velocity per unit electric field and can be extracted from the saturation region of the transfer curve and calculated from its slope using following formula:

$$
I_{d s}=\frac{W C_{i}}{2 L} \mu\left(V_{g s}-V_{t h}\right)^{2}
$$

Where, $\mu$ (field-effect mobility), L (channel length), W (channel width), Ci (capacitance of the dielectric layer), $\mathrm{V}_{\mathrm{g}}$ (gate voltage), and $\mathrm{V}_{\text {th }}$ (threshold voltage). This equation assumes that the mobility of a material is constant. ${ }^{54}$ The parameters 
important for calculating the mobility of OFET device can be extracted from their respective transfer and output characteristics curves (see Figure 1.10).

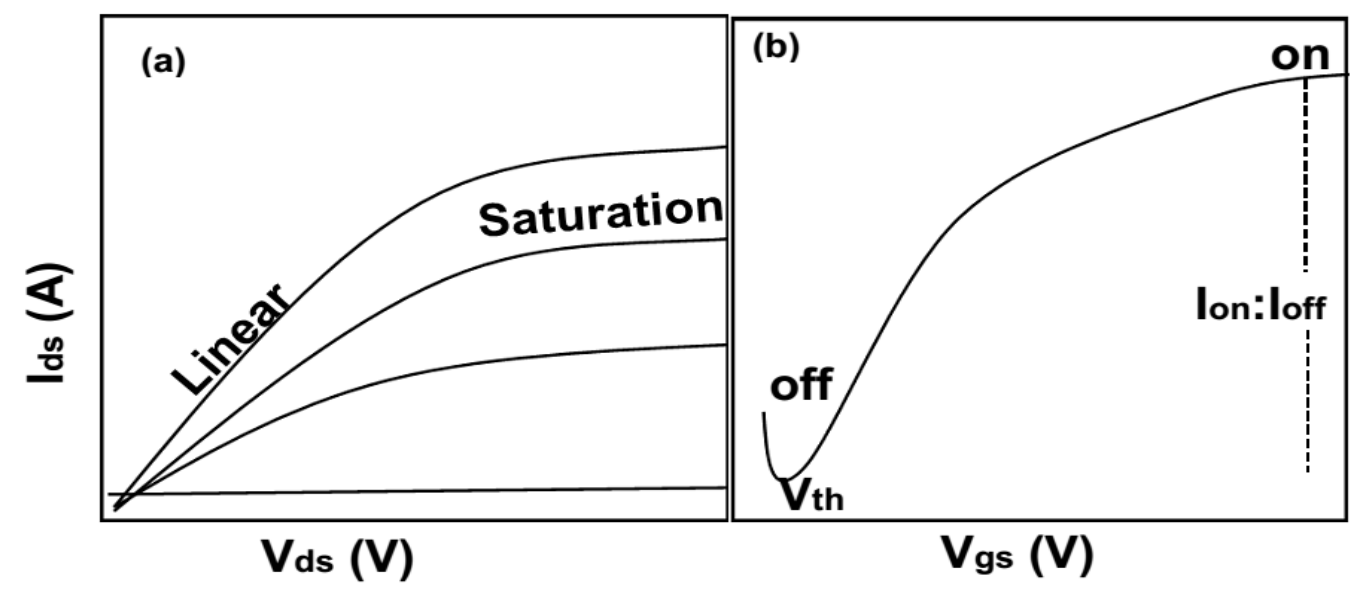

Figure 1.10. The schematic representation for a typical output (a) and transfer curves (b).

In OFET devices, the $\mathrm{I}_{\mathrm{on}} / \mathrm{I}_{\mathrm{off}}$ ratio could be used as an indicator for the purity of organic semiconductor channel from the value of its $I_{\text {off }}$ i.e., the high value of $I_{\text {off }}$ indicates the presence of an extrinsic doping in the semiconducting channel, ideally, it should be zero. For instance, for organic semiconductors to be applied in optoelectronics devices like active matrix displays, it requires high $\mathrm{I}_{\mathrm{on}} / \mathrm{I}_{\text {off }}$ ratio above $10^{6}$ and carrier mobility above $0.1 \mathrm{~cm}^{2} \cdot \mathrm{V}^{-1} \cdot \mathrm{s}^{-1} .55$

\subsubsection{Conjugated polymer materials design for OFET device}

Charge carrier mobility is an important parameter for measuring the performance of organic semiconductors. Research in the organic semiconducting materials for OFET application is towards getting a material which possesses high 
mobility and stability. As charge carriers move in the polymer from orbital to orbital without any hindrance, the carrier mobility is expected to be higher. This can be guaranteed by a high intermolecular p-orbital overlap and good packing (arrangement) or $\pi-\pi$ stacking of conjugated polymers. The intermolecular interaction among the polymer chains could be increased via incorporating fused ring organic molecules and heteroatoms in the polymer backbone, thus it could enhance properties like charge transport and light absorption by suppressing the inter-annular rotation in the conjugated polymer. In addition to this, solution processability of conjugated polymers could be modified by introducing a long alkyl group in the polymer backbone. The relative position of the channel organic semiconductor energy level (LUMO/HOMO) with respect to the work function of source-drain electrodes has greatly affected the charge injection process from or to the organic semiconductor. Therefore, the synthesis and design of conjugated polymers for OFET should take all these factors into consideration.

Based on the majority of charge carriers flowing in or out of the channel, organic semiconductors could be categorized into three. That is, p-type (for hole), ntype (for an electron) and ambipolar (for both hole and electron) charge carriers in the channel under different gate bias conditions (see Figure 1.11). In p-type semiconductors, the HOMO energy level should be in between of -5 to $5.5 \mathrm{eV}$, because most of the conjugated polymer semiconductors are easily oxidized upon exposure to air (for HOMO energy level more than $-5 \mathrm{eV}$ ). Thus, it affects the 
ambient stability and $I_{o n} / I_{\text {off }}$ ratio of the OFET devices. For large HOMO level (less than $-5.5 \mathrm{eV}$ ) could result in OFETs with the high mobility and $I_{o n} / I_{\text {off }}$, but it could cause unacceptably large threshold voltage.
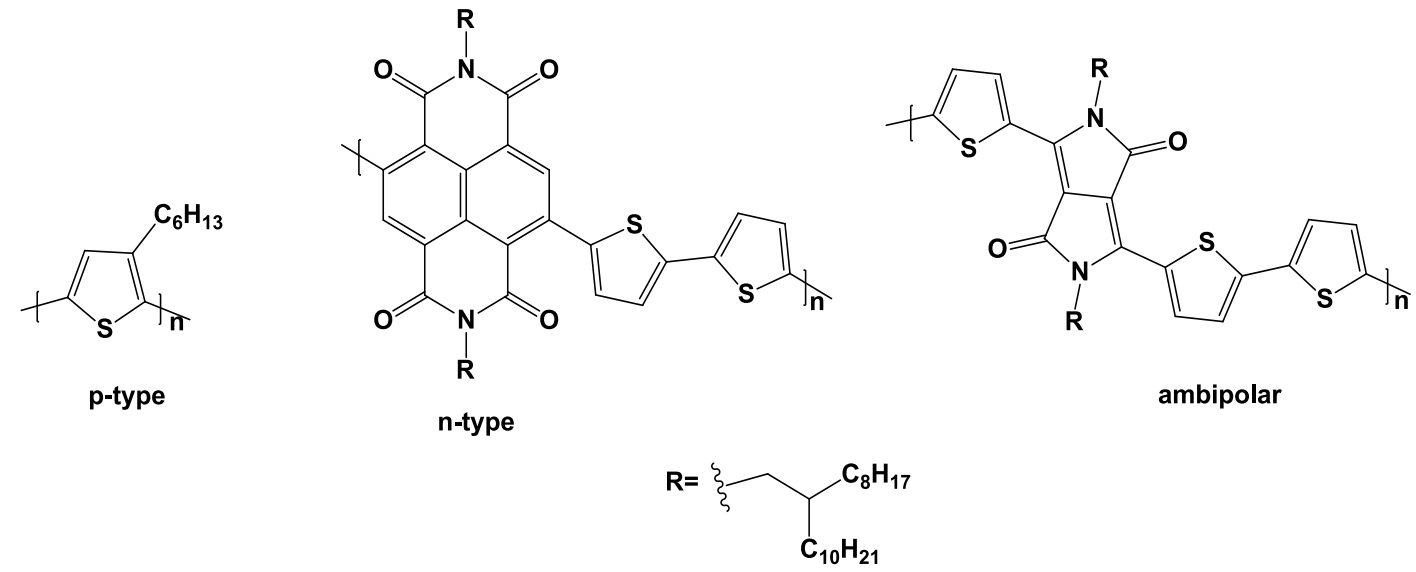

Figure 1.11. Examples of the chemical structure of p-type, ${ }^{56-58}$ n-type, ${ }^{59}$ and ambipolar $^{60}$ semiconductors.

\subsection{Synthetic Route of Conjugated Polymers}

Conjugated polymers are becoming an important component of organic electronics devices as semiconducting material. Therefore, it's quite important to synthesize new $\pi$-conjugated semiconducting polymers and also the development of a new synthetic protocol to realize the commercialization of organic electronics devices.

The single bond between unsaturated carbon atoms could be created via different crosscoupling reactions. Thus, mostly conjugated polymers for organic electronics applications are synthesized via a classical Palladium-catalyzed 
crosscoupling reactions, such as Migita-Kosugi-Stille and Suzuki-Miyaura. As shown in Figure 1.12, oxidative addition of the Palladium catalyst across the Ar-X $(\mathrm{X}=\mathrm{Br}, \mathrm{I})$ bond of an electrophile group is the first step in the catalytic cycle, followed by transmetalation of Ar'-M (arylene diboronic acids/diboronic esters for Suzuki crosscoupling or distannyl arylenes for Stille crosscoupling) of an organometallic nucleophile. Finally, the $\mathrm{C}-\mathrm{C}$ coupling is completed after the reductive elimination step of the catalytic complex. ${ }^{61}$ Recently, a new coupling reaction, direct $\mathrm{C}-\mathrm{H}$ arylation coupling is developed and used in the synthesis of $\pi$-conjugated polymers for organic electronics application. This coupling reaction involves reaction between an aryl halide and another arene monomer directly without the need for preactivation of the C-H bonds, where the need for preparing high-purity organometallic reagents such as arylene diboronic acid (in Suzuki), distannyl arylenes (in Stille) using toxic and flammable precursors makes direct $\mathrm{C}-\mathrm{H}$ arylation coupling as greener and economical alternative coupling method for synthesizing $\pi$-conjugated semiconducting polymers. 


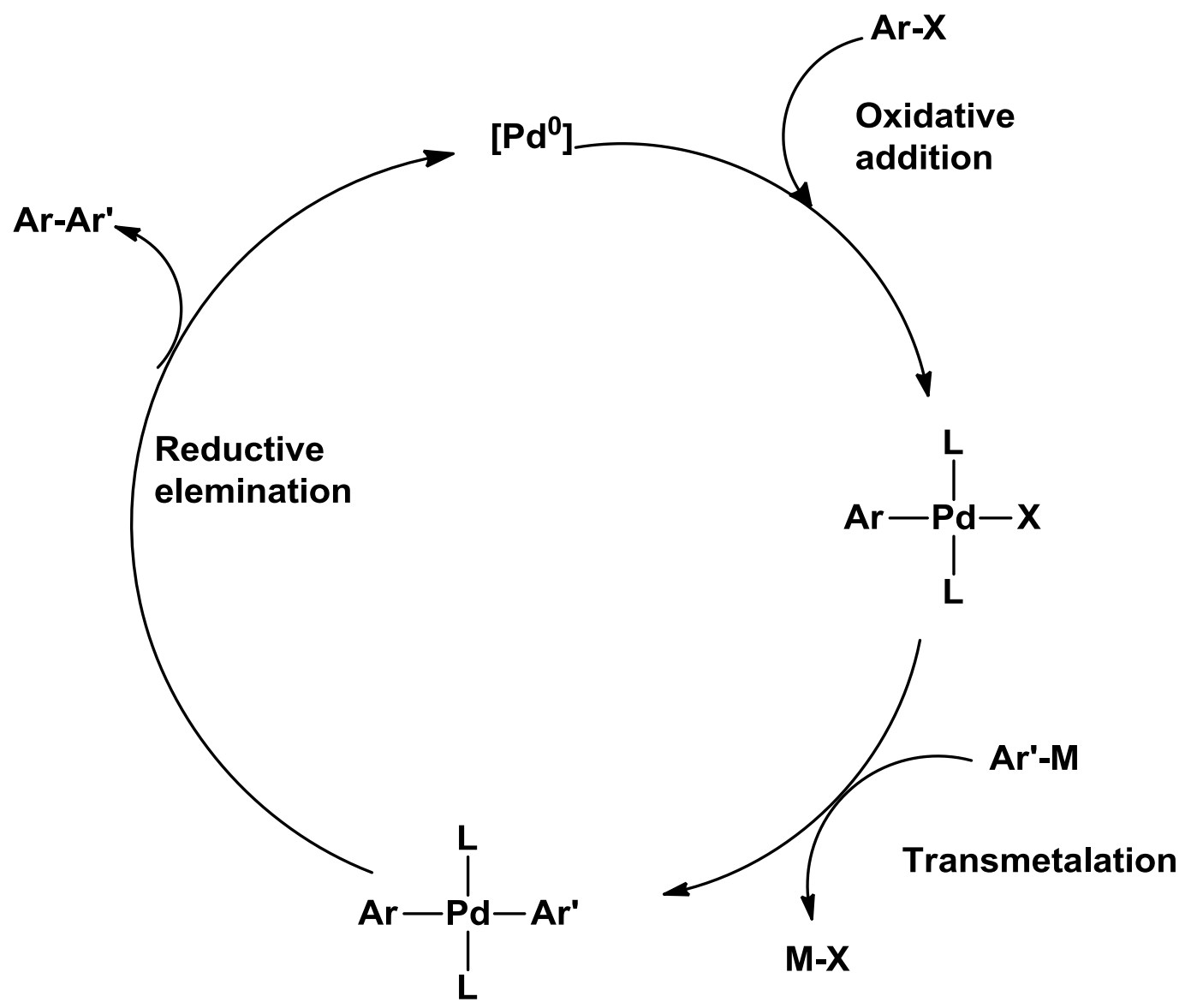

Figure 1.12. Illustration for catalytic cycle of Palladium-catalyzed crosscoupling reactions.

\subsubsection{Stille cross-coupling reaction}

Since the discovery of Stille coupling reaction by Migit, Kosugi, Stille and coworkers from 1976-1978, it has become the most useful protocol for making C-C bonds. ${ }^{62-66}$ The reaction mechanism is depicted in Figure 1.12 , where $\left[\mathrm{Pd}^{0}\right]$ is the active catalyst used, in some case $\left[\mathrm{Pd}^{+2}\right]$ is also used but later it should be reduced to $\left[\mathrm{Pd}^{0}\right]$ to enable the entry of organostannane monomers into the catalytic cycle. ${ }^{67}$ The 
most commonly used $\left[\mathrm{Pd}^{0}\right]$ catalyst is $\mathrm{Pd}\left(\mathrm{PPh}_{3}\right)_{4}$, where the ligand $\mathrm{PPh}_{3}$ can be easily oxidized by traces amount of oxygen in the reaction system to its oxide, $\mathrm{Ph}_{3} \mathrm{PO}$ and also excess $\mathrm{PPh}_{3}$ can inhibit the Stille reaction process. ${ }^{68}$ Therefore, researchers come up with another air stable $\left[\mathrm{Pd}^{0}\right]$ catalyst, $\mathrm{Pd}_{2}(\mathrm{dba})_{3}$ which uses a co-ligand Tri(otolyl)phosphine to promote its kinetics in Stille couplings. The commonly used solvents for Stille coupling reactions to synthesize conjugated polymers are toluene which could help to solubilize the polymer and also highly polar solvents like DMF could help to solubilize the polymer but the solubility of the polymer could be lower than in toluene. Therefore, a mixed solvent system like toluene/DMF could work better for obtaining high molecular weight polymers. ${ }^{69}$

Electron rich monomers like thiophene containing compounds can be easily functionalized by organotin reagents and facilitate the transmetalation step. The advantages of Stille coupling is its high degree of tolerance to many functional groups, however, organotin monomers are less sensitive to oxygen and moister. Therefore, it's broadly applicable to the synthesis of many D-A conjugated polymers. Some of the Stille coupling disadvantages are like the difficulty of synthesizing highly pure organotin compounds and the toxicity of trialkyl tin precursors.

\subsubsection{Suzuki-Miyaura coupling reaction}

In 1979 Suzuki and co-workers have invented a crosscoupling reaction which was later named as Suzuki-Miyaura crosscoupling reaction. ${ }^{70-75}$ Since then it has been developed and used as a good alternative to Stille coupling for the synthesis of 
conjugated semiconducting polymers. ${ }^{76}$ The $\left[\mathrm{Pd}^{0}\right]$ catalytic cycle of Suzuki coupling reaction is more or less similar with Stille where oxidative addition is often the ratelimiting step and the relative reactivity of aryl halides decreases in the order $\mathrm{I}>\mathrm{Br}>$ $\mathrm{Cl}$. The transmetalation of the boronic acid is facilitated by using base (like $\mathrm{K}_{3} \mathrm{PO}_{4}$, $\mathrm{K}_{2} \mathrm{CO}_{3}, \mathrm{NaHCO}_{3}, \mathrm{KOH}, \mathrm{KF}$, and sodium tert-butoxide) by forming a more reactive boronate species that can interact with the $\mathrm{Pd}$ center and transmetalate in an intramolecular fashion. ${ }^{77}$ Because of the base, most Suzuki coupling reactions are carried out in biphasic mixtures of an aqueous phase containing base and organic solvents such as THF, toluene, dioxane, or xylene.

Suzuki polycondensations are carried out mainly between aryl boronic acids (esters) and aryl halides, where aryl boronic acids precursors are more reactive than their ester analogs though it's less stable. Benzene ring containing monomers are more feasible under Suzuki coupling reactions. The advantages like its tolerance to a broad range of functional groups, mild reaction conditions, and the tolerance towards the presence of water and heat make Suzuki coupling reactions more attractive. Whereas, factors like the requirement of basic conditions and its biphasic reaction system could are the drawback of Suzuki coupling reactions.

\subsubsection{Direct $\mathrm{C}-\mathrm{H}$ arylation coupling reaction}

The classical coupling chemistry (such as Stille and Suzuki couplings) requires the preparation of organometallic precursors (like organotin or organoborane monomers) for the synthesis of $\pi$-conjugated semiconducting polymers. As 
described in the previous two sections (1.4.1 and 1.4.2), Stille and Suzuki coupling requires multistep reaction, tedious purification, and toxicity concern makes researchers search for an alternative chemistry. Therefore, direct $\mathrm{C}-\mathrm{H}$ arylation coupling, with the advantages of atom economy, synthetic simplicity, the absence of troublesome and toxic aryl organometallic intermediates makes it be one of the ideal routes toward the synthesis $\pi$-conjugated semiconducting polymers and also for their future large scale production.

Since the report made by Fagnou and coworkers, a number of conjugated polymers have been synthesized and reported in literature. ${ }^{78-80}$ Mechanistically, the direct C$\mathrm{H}$ arylation coupling is a Heck-type coupling reaction and concerted metalationdeprotonation (CMD). ${ }^{81,82}$ The CMD catalytic pathways are still debated, as Fagnou and coworkers proposed two CMD catalytic cycles. The activation of $\mathrm{C}-\mathrm{H}$ bond is the rate limiting step. The oxidative addition of aryl halide (Ar-X) could proceed with or without carboxylate additive (mostly pivalic acid) followed by simultaneous transmetalation-deportation of the arene monomer by the created complex together with the base, then reductive elimination provides aryl-aryl coupled product.

In this type of coupling catalysts $\operatorname{Pd}(\mathrm{OAc})_{2}, \operatorname{Pd} 2(\mathrm{dba})_{3}$, and highly stable Herrmann-Beller catalysts are commonly employed together with an appropriate combination of solvent (polar solvents like DMAc, DMF, NMP, THF and nonpolar solvent toluene, o-xylene) and supporting phosphine ligands. However, serious of 
optimization steps among catalyst, solvent, ligand, concentration, and reaction time needed to obtain a suitable combination towards high molecular weight and good quality conjugated polymer for organic electronics application. The drawbacks for this coupling method is like poor selectivity of $\mathrm{C}-\mathrm{H}$ bond which leads to branching and crosslinking of the polymer that affects the solubility and its optoelectronic properties. $^{83,84}$

(a)


(b)
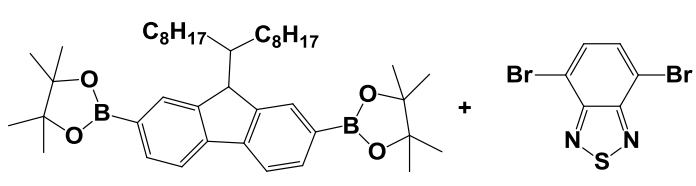

$\underset{\text { Suzuki-Miyaura }}{\text { coupling }}$

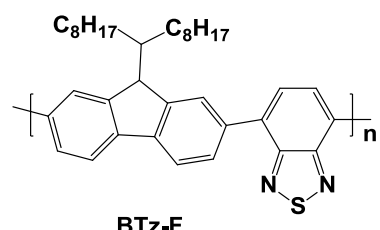

(c)
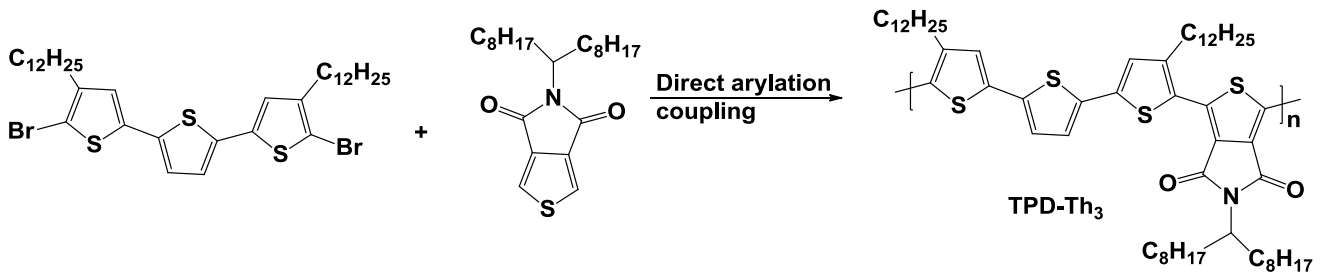

Figure 1.13. Representative synthesis Scheme of $\pi$-conjugated polymer (PTB7) by Stille (a) ${ }^{85}$, BTz-Th3 by Suzuki-Miyaura (b) ${ }^{86}$ and TPD-Th3 by direct arylation coupling method (c).$^{87}$ 


\subsection{Donor-Acceptor Conjugated Polymers for Organic Electronics Applications (OPVs and OFETs)}

Since the discovery of conductive polymers in 1977 by Heeger, MacDiarmid, and Shirakawa ${ }^{3}$ a significant progress has been made in the synthesis of conjugated polymers and their applications for optoelectronic devices such as organic photovoltaics (OPVs), organic field effect transistors (OFETs), and organic lightemitting diodes (OLEDs). In the past decades, a huge number of conjugated polymers have been synthesized and applied to different types of organic electronic devices. So the progress specifically related to BHJ-OPV and OFET applications is summarized below.

Earlier researchers were centered on materials based on poly(phenylene vinylene) (PPV) and its derivative as a primary light-absorbing the material in BHJOPVs. In 2001, Shaheen et al. ${ }^{88,89}$ developed bulk heterojunctions of poly[2methoxy-5-(2'-ethylhexyloxy)-1,4-phenylenevinylene] (MEH-PPV) with PCBM to produce solar cells with $2.5 \%$ PCE, the highest reported efficiencies for polymeric solar cells at the time. ${ }^{88,89}$ Much of the improvement comes from the use of high concentration of PCBM and also using chlorinated solvent. Despite the impressive initial 2.5\% PCE shown by Shaheen et al., further improvement in PPV-PCBMbased bulk heterojunctions only allowed for modest increases in PCE values of 3 to $3.3 \%$. This type of polymers was characterized by large band gap which leads to high 
$\mathrm{V}_{\mathrm{oc}}$ up to $0.82 \mathrm{~V}$ and thus it limits $\mathrm{J}_{\mathrm{sc}}$ as low as $5-6 \mathrm{~mA} / \mathrm{cm}^{2}$ due to the lack of absorption of low energy photons. ${ }^{88,89}$

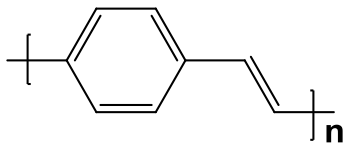

PPV

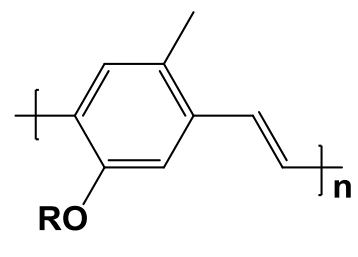

MEH-PPV

Figure 1.14. PPV and its derivative type conjugated polymers for OPV applications.

The second phase of donor polymer research was mainly focused on designing and synthesizing low band gap conjugated polymer to enhance the value of $\mathbf{J}_{\mathrm{sc}}$ by absorbing low energy photons. This motivation has led to the development of P3HTPCBM based OPVs, ${ }^{90}$ where it was mainly characterized as a low band gap $(1.9 \mathrm{eV})$, a high degree of crystallinity and mobility polymer. Thus, P3HT-PCBM BHJs provide OPVs with $\mathrm{J}_{\mathrm{sc}}$ of $10 \mathrm{~mA} / \mathrm{cm}^{2}{ }^{91-93}$ and PCE of $\sim 5 \%$, together with other optimization techniques like thermal ${ }^{93}$ or solvent annealing. ${ }^{94}$ Unfortunately, its high-lying HOMO energy level causes low $\mathrm{V}_{\text {oc }}(\sim 0.6 \mathrm{~V})$ value of the resulting OPV.

The polythiophenes like P3HT has been also extensively studied for OFETs with different regioregularity arrangement to give the first solution-processed OFET with hole mobility of $10^{-4}-10^{-5} \mathrm{~cm}^{2} \mathrm{~V}^{-1} \mathrm{~s}^{-1}$ for regiorandom $\mathrm{P} 3 \mathrm{HT} 95$ and then by controlling regioregularity of P3HT with edge-on orientation, it could have been possible to improve the mobility to $0.05-0.2 \mathrm{~cm}^{2} \mathrm{~V}^{-1} \mathrm{~s}^{-1} \cdot{ }^{96-98}$ The stability issue of P3HT based OFET device was addressed by Ong et al., where regioregular 
quaternary thiophene (PQT) has been synthesized to give mobility of $0.14 \mathrm{~cm}^{2} \cdot \mathrm{V}^{-1} \cdot \mathrm{s}^{-}$ 199

The third phase conjugated polymer was mainly focused on synthesizing copolymers incorporating an electron donor (D) and acceptor (A) units in their conjugated polymer backbone. ${ }^{100,101}$ Structural studies reveal that intermolecular DA interactions could enable the opportunity to tune various physicoelectrochemical properties of the D-A conjugated polymer via copolymerizing various couples of electron donor and acceptor moieties. ${ }^{102}$ Therefore, semiconducting properties of these type of conjugated polymers are influenced significantly by the characteristics of electron donors and acceptors in the polymeric backbone. ${ }^{103}$ Various electron acceptors have been successfully incorporated into D-A conjugated polymers to yield semiconductors with high carrier mobilities and PCEs. ${ }^{104,105}$ For instance, recently lots of conjugated D-A polymers, containing benzothiadiazole (BTz) and its derivatives, bis-amide-based electron acceptors, such as diketopyrrolopyrrole (DPP), isoindigo and their derivatives, were found to show high semiconducting performances with p-type, n-type and ambipolar behaviors. ${ }^{106-108}$ In addition, a huge number of D-A conjugated polymers have been reported based on the abovementioned acceptor moieties, with some of possessing $\mathrm{V}_{\mathrm{oc}}>1 \mathrm{~V},{ }^{109-111} \mathrm{~J}_{\mathrm{sc}}>17$ $\mathrm{mA} / \mathrm{cm}^{2},{ }^{112,113}$ and $\mathrm{FF}>70 \%{ }^{114,115}$ but many of them are not yet providing PCE $>10 \%$. Therefore, D-A conjugated polymer should be designed with a better understanding to optimize values of $\mathrm{V}_{\mathrm{oc}}, \mathrm{J}_{\mathrm{sc}}$, and FF to obtain high PCE. The search 
of new donor polymer materials was focused on designing and synthesizing a new building block moieties for the construction of D-A type of conjugated polymers. Incorporating fused ring type of building blocks was often chosen due to their rigidly enforced planarity, which could benefit more effective $\pi$-electron delocalization in the conjugated polymer backbone and decreased optical band gaps while improving $\pi-\pi$ interactions between polymer chains in thin films, thereby improving charge carrier mobility in devices.

Another designing strategy of D-A conjugated polymers was introducing an electron withdrawing group like of thiadiazole to the backbone of conjugated polymer have attracted immense research interests, as a result, many D-A based conjugated polymer have been synthesized with majority of them are based on 2,1 , 3- benzothiadiazole (BTz) moiety. ${ }^{116-119}$ Compared to other acceptor moieties, BTz has been ubiquitously utilized to construct D-A polymers due to its high electron mobility and relatively low HOMO energy level, causing high $\mathrm{V}_{\text {oc }}$ in OPVs. ${ }^{120-123}$ Moreover, BTz-based D-A conjugated polymers have been exploited by modifying it through substitution of the sulfur atom with oxygen, ${ }^{124}$ nitrogen, ${ }^{125}$ and selenium, ${ }^{126}$ and by replacing the benzene ring with more electron withdrawing ring like pyridine, ${ }^{127}$ pyridazines. ${ }^{128}$ The exchange of hydrogen atoms with heteroatoms or groups, such as fluorine atoms and alkyloxy side chains have brought so many interesting developments in PCE value of OPVs and charge (electron or hole) mobility in OFETs. In particular, more than 10\% PCE was achieved for OPVs based 
on fluorinated BTz type of D-A conjugated polymer. ${ }^{129,130}$ Another design strategy to modify $\mathrm{BTz}$ based D-A conjugated polymers is the fusion of benzene ring with aromatics rings, this might increase the $\pi-\pi$ stacking and planarity of the resulting copolymer (see Figure 1.15).

In this dissertation work, synthesis of a new fused-ring building block 2,1,3dithienobenzochalcogenodiazole (2,1,3-dithienobenzothiaodiazole and 2,1,3dithienobenzooxa diazole) and its application in the design and synthesis of D-A conjugated polymers for OPV and OFET application is presented (in Chapter 2). Benzo[1,2-b:6,5-b']dithiophene planarity and efficient $\pi-\pi$ stacking with the electron-withdrawing capacity of the chalcogenodiazole functionality serves as the foundation for this new class of materials. The introduction of the $\pi$-electron deficient chalcogenodiazole unit can decrease the HOMO and LUMO energies of the polymers, which is necessary for air-stable polymers.
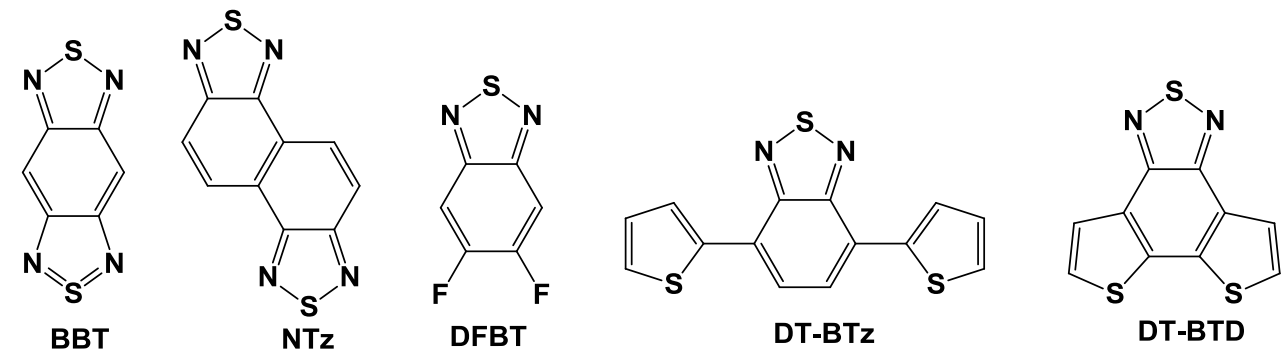

Figure 1.15. Thiadiazole electron withdrawing group containing acceptor moieties.

Another class of acceptor moieties for the construction of D-A conjugated polymers are moieties containing a five-membered lactam-ring (bis-amide), such as 
diketopyrrolopyrrole (DPP), isoindigo, and indigo (see Figure 1.16) which have been extensively studied for high-performance organic electronics. This lactam-ring containing conjugated molecules usually possess planar and polar ring structures, which facilitate intermolecular $\pi-\pi$ stacking and electron donor-acceptor interactions. A number of high performance p-type, n-type, and ambipolar organic semiconductors based on these five-membered lactam-ring moieties have been reported. For instance, high-performance semiconductors were reported based on DPP-containing conjugated polymers: p-type semiconductors with hole mobilities up to $12.04 \mathrm{~cm}^{2} \mathrm{~V}^{-1} \mathrm{~s}^{-1},{ }^{49} \mathrm{n}$-type ones with electron mobilities above $3 \mathrm{~cm}^{2} \mathrm{~V}^{-1} \mathrm{~s}^{-1131}$ and even ambipolar ones with hole and electron mobilities up to $8.84 \mathrm{~cm}^{2} \mathrm{~V}^{-1} \mathrm{~s}^{-1}$ and $4.34 \mathrm{~cm}^{2} \mathrm{~V}^{-1} \mathrm{~s}^{-1}$, respectively. ${ }^{132}$

High charge carrier mobilities were achieved for conjugated polymers based on isoindigo derivatives. For instance, Pei et al. reported an isoindigo-based D-A conjugated polymer with an exceptionally high hole mobility of $0.79 \mathrm{~cm}^{2} \mathrm{~V}^{-1} \mathrm{~s}^{-1}, 133$ which was later improved to $3.62 \mathrm{~cm}^{2} \mathrm{~V}^{-1} \mathrm{~s}^{-1} .{ }^{134}$ Bao et al. synthesized a siloxaneterminated isoindigo-based conjugated polymer with a hole mobility as high as 2.48 $\mathrm{cm}^{2} \mathrm{~V}^{-1} \mathrm{~s}^{-1} \cdot{ }^{135}$ 

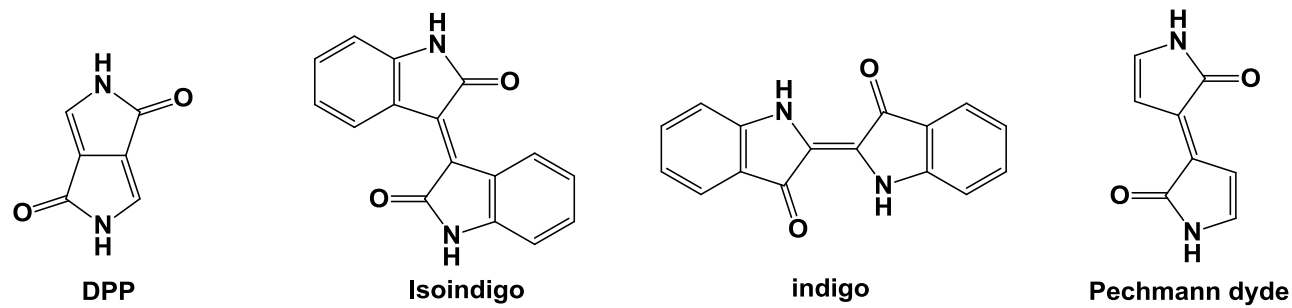

Figure 1.16. Five member lactam-ring containing acceptor moieties.

Structural modification of isoindigo which is a structural isomer of the famous pigment indigo gave a number of new acceptor moieties. For instance, fluorination of the isoindigo unit gave a new acceptor which lower the LUMO level of the corresponding polymer and increase the electron mobility. Replacing the benzene ring with thiophene gives a new acceptor moiety, namely thioisoindigo. It has been copolymerized with many donor moieties. The FET performances from these polymers, however, were moderate compared with the isoindigo-based analogs, reaching hole mobilities between $10^{-4}$ and $10^{-2} \mathrm{~cm}^{2} \mathrm{~V}^{-1} \mathrm{~s}^{-1} .{ }^{136,137}$ Inserting a lactonecontaining electron withdrawing group, benzodifurandione in between of the two oxindole units of isoindigo provides n-type and ambipolar conjugated polymer. ${ }^{138} \mathrm{In}$ addition, recently other electron acceptor moiety with lactam ring, namely Pechmann dye, where the two benzene rings of isoindigo replaced with flanked thiophenes were developed. It has been copolymerized with electron donor moieties and the FET performance of hole mobility $1.37 \mathrm{~cm}^{2} \mathrm{~V}^{-1} \mathrm{~s}^{-1} .139$

In this dissertation work, a new acceptor moiety containing five-member lactone-ring, namely lactone Pechmann dye based D-A conjugated polymers have 
been synthesized and applied to OFET devices (in Chapter 3). The strong electron withdrawing nature of oxygen (in lactone-ring) than nitrogen (in lactam ring) and the absence of alkyl group substitution could potentially reduce the steric hindrance and thus makes it more planar and attractive acceptors moiety than their lactam analogs. In addition to this, the intermolecular hydrogen bonding between the amide proton $(\mathrm{N}-\mathrm{H})$ in the lactam ring of isoindigo and the carbonyl oxygen $(\mathrm{C}=\mathrm{O})$ could be important for an improved charge transfer in its corresponding organic electronics device. Therefore, in this dissertation (in Chapter 4) thermally labile or acid-induced removal of the t-BoC group could free the amide proton and thus it could produce the desirable intermolecular hydrogen bonding and $\pi-\pi$ interaction.

Most of the above mentioned D-A conjugated polymers for organic electronics applications are synthesized by using traditional Palladium-catalyzed cross-coupling reactions, such as Stille, Suzuki, Sonogashira-Hagihara, and Yamamoto couplings. Nonetheless, these coupling methods involve a tedious preactivation of $\mathrm{C}-\mathrm{H}$ bond and thus, they require multistep reactions, tedious purification, and toxicity concerns in preparation of the organometallic precursors (like organotin or organoborane monomers) make them less environmentally benign and uneconomical synthetic methods for the D-A conjugated polymers. However, a new synthesis approach, namely direct $\mathrm{C}-\mathrm{H}$ arylation polymerization (DAP) has attracted a tremendous amount of research interest for synthesizing D-A conjugated polymers, as it is atomically efficient, cheap, and green synthetic method. Therefore, recently a lot of 
D-A conjugated polymers have been synthesized using DAP. For instance, 2,1,3benzothiadiazole,${ }^{83,140-142}$ DPP,${ }^{143-146}$ isoindigo, ${ }^{147-149}$ and thioisoindigo ${ }^{150}$ based D-A conjugated polymers having high performance in OPV and OFET devices have been synthesized. Interplay among different catalytic conditions such as, catalyst, ligand, base, solvent,

Interplay among different catalytic conditions such as, catalyst, ligand, base, solvent, concentration, reaction time and temperature, has led to optimized DAP reaction towards high molecular weight polymer with less defect from homocoupling and beta-branching in the polymer backbone. ${ }^{83,84,144,151-153}$ Therefore, in this dissertation, high-performance 5,6-difluro-2,1,3-benzothidiazole based D-A conjugated polymers have been successfully synthesized and applied to OPV and OFET devices (in Chapter $5 \& 6$ ). This synthetic method has been less explored in the synthesis of higher dimensional conjugated polymer networks, such as conjugated microporous polymers (CMPs). Therefore, it has been extended to the synthesis of CMPs (in Chapter 7).

\subsection{Motivation and Scope of the Thesis}

Over the last three decades, research in the organic electronics devices has grown rapidly in response to the demand for cheap and flexible electronics device for applications in renewable energy source and modern microelectronics. The thin films of $\pi$-conjugated organic semiconductors are used as an active layer in the organic electronics devices (like OFETs, OPVs). In OPVs, the active layer which is 
mainly responsible for its performance is mainly composed of $\pi$-conjugated polymer/fullerene (PCBM) in bulk heterojunction (BHJ) configuration. Therefore, the design and synthesis of conjugated polymers having characteristics like low band gap, high charge carrier mobility, good solubility, proper alignment of its energy levels (HOMO, LUMO) and control of its thin film morphology have been important elements in the research and development of organic electronics devices. One of the most common and successful design strategies in tailoring the physicoelectrochemical properties of conjugated polymers is a copolymerization of the donor (electron rich) and acceptor (electron deficient) moieties together. The number of acceptor moieties available for choice is quite small as compared with donor moieties. Therefore, designing and synthesizing a new acceptor moiety and its corresponding donor-acceptor conjugated polymer is quite important to further extend the development of organic electronics devices and then realize their commercialization.

One of the design strategies to increase the carrier mobility and also tailoring HOMO/LUMO levels is via incorporating fused aromatic compounds into the polymer backbone as it restricts the intramolecular rotation and also promotes $\pi-\pi$ stacking (intermolecular interactions) among the polymer chains. This phenomenon could also be promoted via incorporating heteroatoms like chalcogens (oxygen, sulfur, and selenium). In addition to this, finding a novel acceptor building blocks 
also crucial in the realm of synthesizing new donor-acceptor conjugated polymers and thus high-performance OFETs and OPVs devices.

Most of D-A conjugated polymers are synthesized via traditional coupling techniques (such as Stille and Suzuki coupling reactions), which needs a tedious preactivation of $\mathrm{C}-\mathrm{H}$ bond by using toxic and flammable precursors to make their respective nucleophilic monomers (organostannane and organoborane). Therefore, an alternative coupling method called direct $\mathrm{C}-\mathrm{H}$ arylation coupling attracts a lot of attention as there is no requirement for preactivation of $\mathrm{C}-\mathrm{H}$ bond with organometallic reagents, which makes it an economical and greener synthesis method and will be suitable for future large-scale synthesis of high quality conjugated polymers. Given this impetus, the scope of this dissertation focuses on:

I. Designing and synthesizing novel donor-acceptor conjugated polymers for applications in organic electronics devices like OFETs and OPVs.

- Synthesis of thiophene fused 2,1,3-benzochalcogenodiazole acceptor moiety and its corresponding donor-acceptor conjugated polymers via Stille and Suzuki polycondensation.

- Investigate the effect of chalcogen atom exchange in the physicoelectrochemical properties of the copolymers. 
- Synthesis of novel acceptor moiety, $\gamma$-lactone-Pechmann dye and its corresponding donor-acceptor conjugated polymers via Stille polycondensation.

- Synthesis of t-BoC-Isoindigo based conjugated polymers and investigating the effect of latent side group (t-BoC) removal on its physicoelectrochemical properties.

- Investigate the synthesized donor-acceptor conjugated polymers performance in organic electronics devices (such as OFETs and OPVs).

II. Exploring the synthesis of donor-acceptor conjugated polymers via direct arylation polymerization (DAP)

- Synthesis and characterization of 5,6-difluoro-2,1,3benzothiadiazole-alt-quternery thiophene conjugated polymer via DAP

- Synthesis and characterization of 5,6-difluoro-2,1,3benzothiadiazole-alt-5H-dithieno[3,2-b:2',3'-d]pyran via DAP

- Investigate the synthesized donor-acceptor conjugated polymers performance in organic electronics devices (such as OFETs and OPVs).

III. Expanding the direct arylation polymerization (DAP) synthesis method to other types of conjugated polymers 
- Synthesis and characterization of conjugated microporous polymers (CMPs) via direct arylation polymerization (DAP)

In this dissertation, the design and synthesis of novel donor-acceptor conjugated polymers via both traditional (Stille and Suzuki couplings) and direct arylation polymerization methods are presented. The potential of direct C-H arylation coupling to the synthesis of other multidimensional polymers is also explored. The structures and properties of the synthesized materials have been fully characterized and investigated for applications like OFETs and OPVs. In addition, an outline for possible future work is suggested in the last Chapter of this dissertation. 


\section{CHAPTER 2}

\section{Dithienobenzochalcogenodiazole-Based Electron}

\section{Donor-Acceptor Polymers for Organic Electronics}

\subsection{Introduction}

Research on organic semiconductors has advanced rapidly over the past decade owing to their potential as electrically active materials in producing flexible, large area, light weight and low-cost electronic devices such as organic light-emitting diodes (OLEDs), organic photovoltaic (OPVs), and organic field-effect transistors (OFETs). ${ }^{104,154,155}$ Structurally, organic semiconductors can be readily modified to tune molecular properties such as crystallinity, energy levels, optical band gap, etc. to achieve desirable electrical performance characteristics. ${ }^{156-159}$ Thus, understanding the relationships between molecular structure, material properties, and device performance would enable rational materials design for improved device performance. From these perspectives, conjugated electron donor and acceptor (DA) polymers have been a plausible structural design strategy to achieve the required materials properties through controlled intramolecular charge transfer from the donor to acceptor moiety within the polymer framework. ${ }^{160}$ 
Chalcogenodiazole chromophore has received great interest as an electron acceptor moiety for the construction of D-A polymers for organic electronics applications. Many known D-A polymers utilize 2,1,3-benzothiadiazole (BTz) structure, ${ }^{161-164}$ which has high electron affinity and relatively low HOMO level as compared to other acceptor moieties. ${ }^{165-168}$ D-A polymers with the BTz moiety modified via substitution of sulfur with other heteroatoms have led to interesting DA systems, offering enhanced OPVs and OFET performance characteristics. ${ }^{169-173}$ Using BTz-based D-A polymers as donors, Liu et al. has recently reported PCE as high as over $10 \%$ of OPV devices. ${ }^{129}$

Another design modification of the BTz moiety in D-A polymers is through fusion of its benzene ring with other aromatics groups to maximize $\pi$-orbital overlap by restricting intramolecular rotation such as to induce optimal face-to-face $\pi-\pi$ stacking and thus to facilitate charge transport via intermolecular hopping. ${ }^{174-177}$ Thus, some of the recent works on fusion of BTz moiety with thiophene, indole, and thieno[3,2-b]pyrrole have shown significant improvements in physical, electrochemical and device properties of the resulting polymers. ${ }^{178-185} \mathrm{D}$-A polymers derived from another acceptor structure, benzooxadiazole $(\mathrm{BOz})$ have also been shown to exhibit enhanced electronic device performance. ${ }^{186-193}$ To the best of our knowledge, few D-A small molecules and polymers based on thiophene-fusedbenzooxadiazole have been reported for organic electronics applications. ${ }^{194,195}$ 
This Chapter presents the synthesis and optoelectronic characterization of five D-A polymers based on two acceptors, dithieno[3',2':3,4;2",3":5,6]benzo[1,2c][1,2,5]thiadiazole (DT-BTD) and dithieno[3',2':3,4;2",3":5,6] benzo [1,2-c][1,2,5] oxadiazole (DT-BOD), alternating with three donor moieties, thienothiophene (TT), bithiophene (BT), and alkylidenefluorene. ${ }^{196}$ We also studied the effects of chalcogen atoms ( $\mathrm{S}$ and $\mathrm{O}$ ) on their physicochemical properties and OPV and OFET device performances.

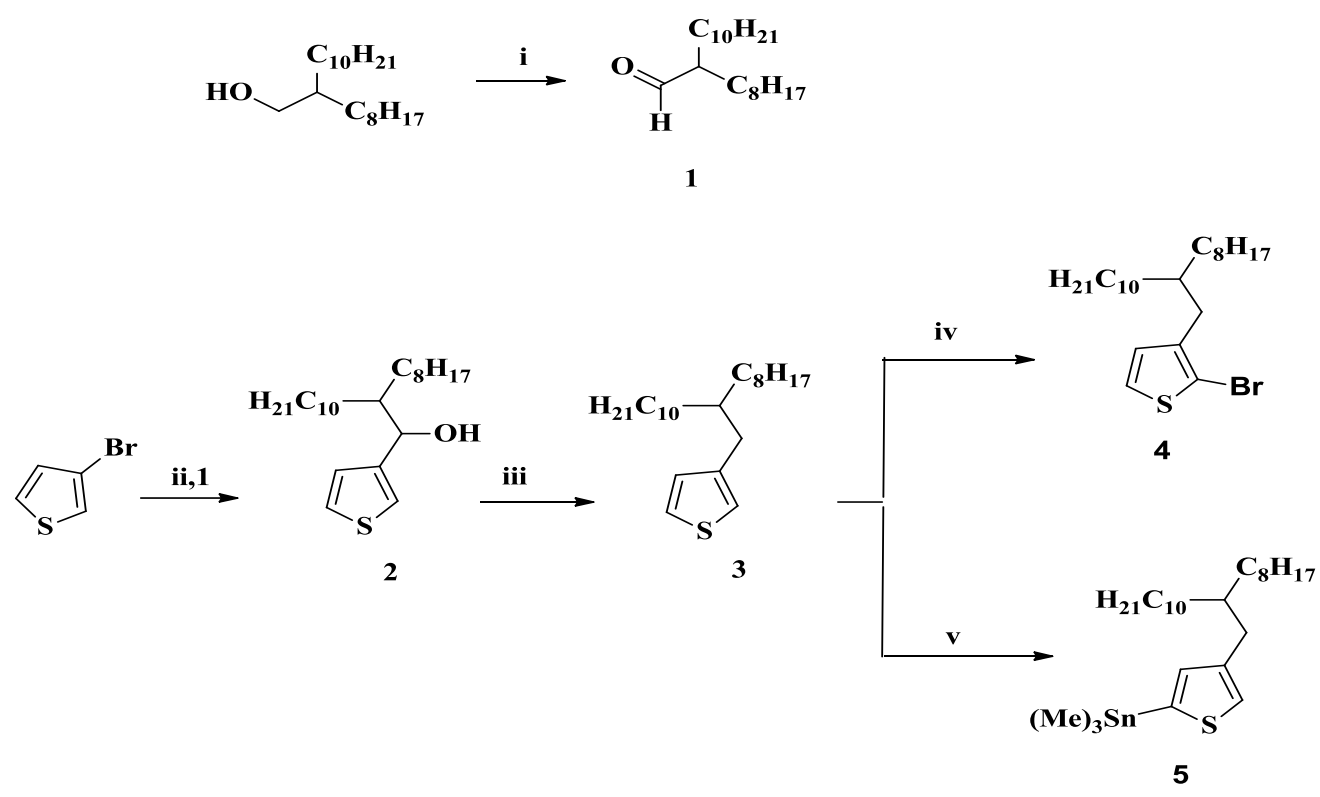

Scheme 1: Synthesis of intermediate 4 and 5: (i) $\mathrm{PCC}, \mathrm{CH}_{2} \mathrm{Cl}_{2}$, r.t, 90\%. (ii) $i$ PrMgCl-LiCl, THF, r.t. (iii) $\mathrm{LiAlH}_{4} / \mathrm{AlCl}_{3}, \mathrm{Et}_{2} \mathrm{O}$, r.t, 50\%. (iv) NBS, DMF, r.t, $80 \%$. (v) $\mathrm{Sn}(\mathrm{Me}){ }_{3} \mathrm{Cl}, \mathrm{THF},-78{ }^{\circ} \mathrm{C}, 96 \%$. 


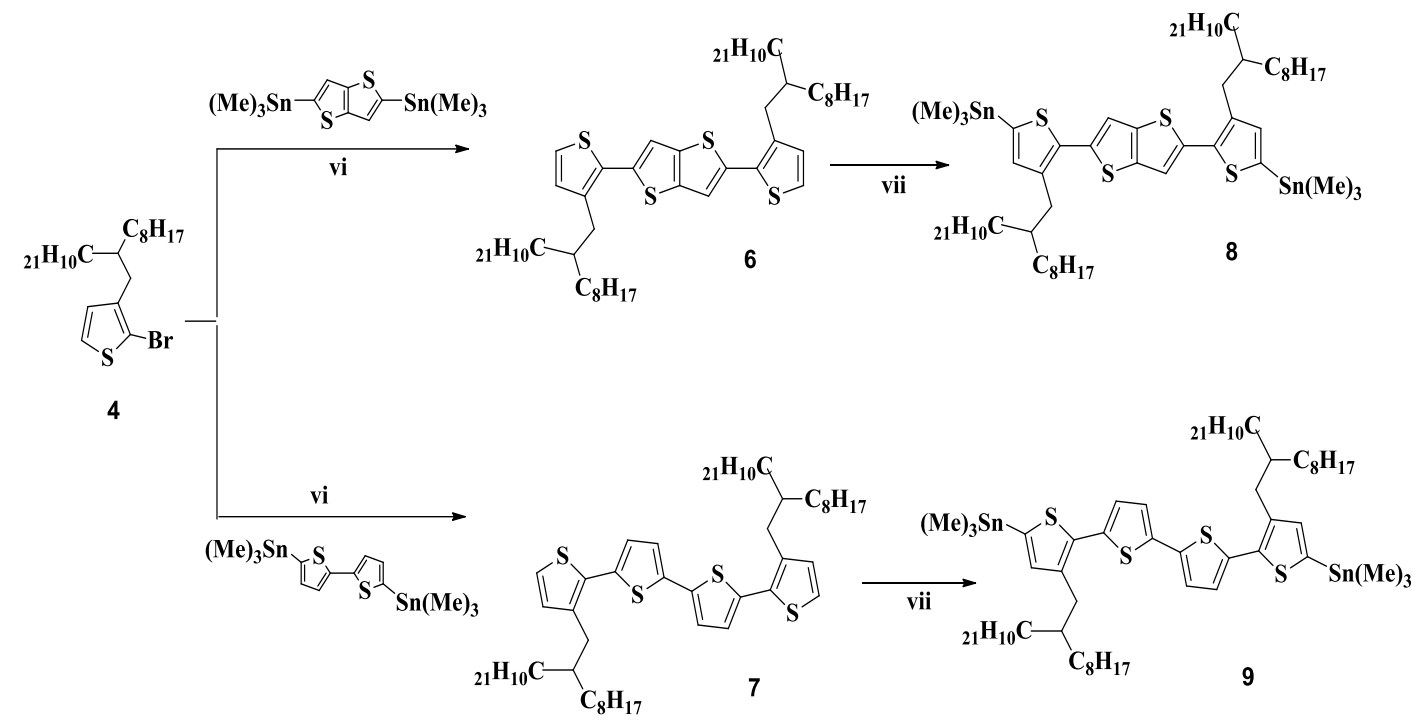

Scheme 2: Synthesis of donor moieties, Compound 8 and 9. (vi) $\mathrm{Pd}_{2}(\mathrm{dba})_{3} / \mathrm{P}(\mathrm{o}-$ tolyl) $)_{3}$, THF, reflux, $44 \%$ for 6 and $41 \%$ for 7. (vii) (Me) $)_{3} \mathrm{SnCl}$, THF, r.t, $88 \%$ for 8 and $70 \%$ for 9.
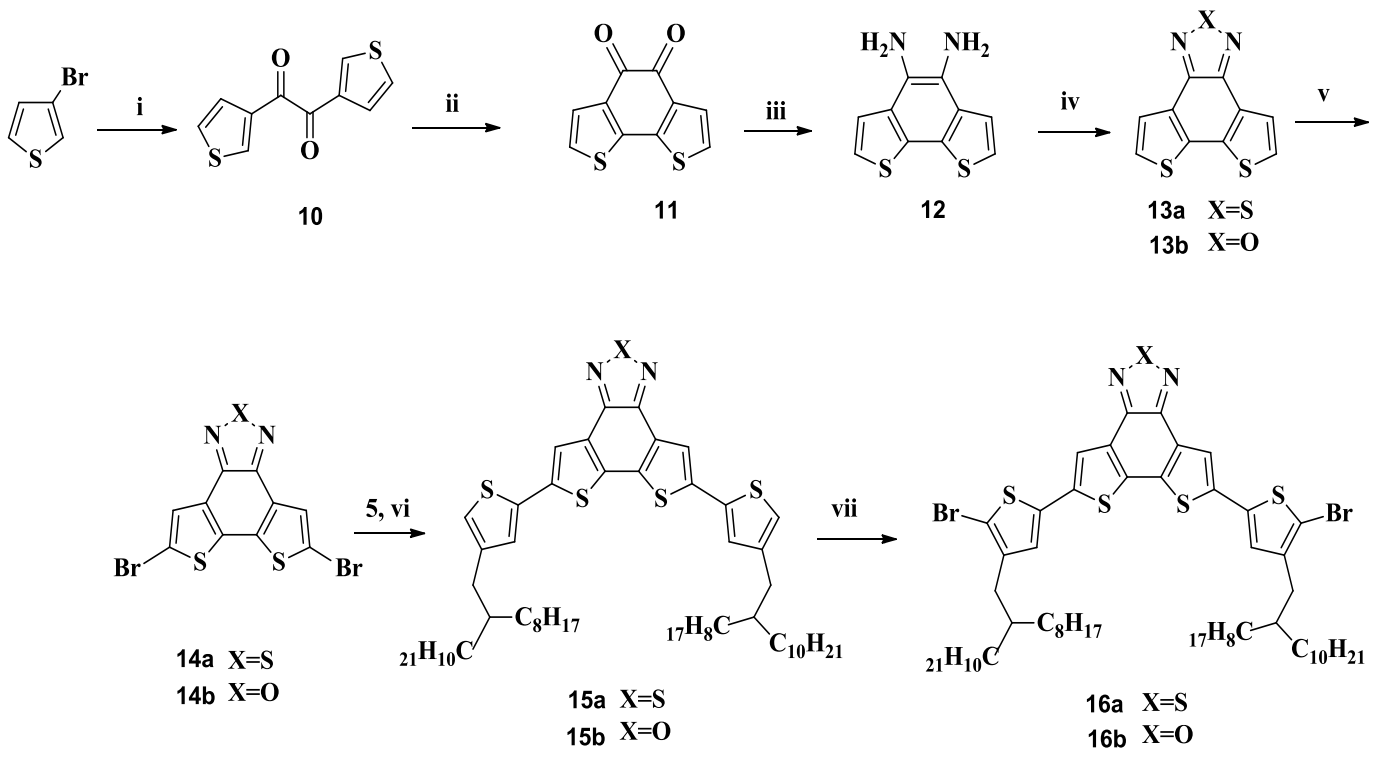

Scheme 3: Synthesis of acceptor monomers 16a and 16b. (i) n-BuLi, $-78{ }^{\circ} \mathrm{C}$, $\mathrm{CuBr} / \mathrm{LiBr}$ then oxalyl chloride, THF, 78\%. (ii) $\mathrm{FeCl}_{3}, \mathrm{CH}_{2} \mathrm{Cl}_{2}$, r.t, 82\%. (iii) 13a) 
$\mathrm{HONH}_{2} \cdot \mathrm{HCl}, 10 \% \mathrm{Pd} / \mathrm{C}, 78{ }^{\circ} \mathrm{C}$ then $\mathrm{N}_{2} \mathrm{H}_{4} \cdot \mathrm{H}_{2} \mathrm{O}, \sim 60{ }^{\circ} \mathrm{C}, \mathrm{EtOH}, 34 \%$. (iv)13a) $\mathrm{SOCl}_{2}, \mathrm{Et}_{3} \mathrm{~N}, \mathrm{DCM}$, reflux, 79\%. (iii) $\left.\mathbf{1 3 b}\right) \mathrm{HONH}_{2} \cdot \mathrm{HCl}, 85^{\circ} \mathrm{C}$ for $24 \mathrm{~h}$. (iv) $\mathbf{1 3 b}$ ) $140{ }^{\circ} \mathrm{C}$ for $60 \mathrm{~h}, \mathrm{EtOH}, 30 \%$. (v) $\mathrm{Br}_{2}, \mathrm{CHCl}_{3}$, reflux, $75 \%$ for $\mathbf{1 4 a}$ and $69 \%$ for $\mathbf{1 4 b}$. (vi) $\mathrm{Pd}\left(\mathrm{PPh}_{3}\right)_{4}$, toluene, $110{ }^{\circ} \mathrm{C} .71 \%$ for $\mathbf{1 5 a}$ and $84 \%$ for $\mathbf{1 5 b}$ (vii) NBS, THF, r.t. $70 \%$ for $\mathbf{1 6 a a n d} 92 \%$ for $\mathbf{1 6 b}$.



Scheme 4: A previous synthetic route to D-A polymers, P1 and P2. (i) $\mathrm{Pd}\left(\mathrm{PPh}_{3}\right)_{4}$, toluene, $110{ }^{\circ} \mathrm{C}, 54.5 \%(\mathbf{P 1})$ and $27 \%(\mathbf{P 2})$ yield.

\subsection{Experimental}

Materials. Unless stated otherwise, all starting materials were obtained from Sigma Aldrich and used without further purification. The anhydrous solvents like 
tetrahydrofuran (THF), diethyl ether, and toluene for the reaction were collected from PureSolv MD solvent purification system of model PS-400-5-MD. Compound (3, and 6),${ }^{197}$ Compound $\left(\mathbf{1 0}, \mathbf{1 1}\right.$, and 12) ${ }^{198}$ and Compound (13a) ${ }^{199}$ were prepared according to reported procedures. All the reactions were carried out under an inert atmosphere.

\subsubsection{Synthesis of monomers}

\section{2-Octyldodecanal (1)}

To $250 \mathrm{~mL}$ round-bottom flask, 2-octyldodecanol (10.056 g, $33.68 \mathrm{mmol}, 1$ equiv), $\mathrm{MgSO}_{4}(20 \mathrm{~g}), \mathrm{CH}_{2} \mathrm{Cl}_{2}(120 \mathrm{~mL})$, was added under nitrogen environment and stirred for a few seconds and followed by adding pyridiumchlorochromate (PCC) (10.84 g, $50.524 \mathrm{mmol}, 1.5$ equiv) to the mixture and stirred at room temperature

for $12 \mathrm{~h}$. After the reaction completed it was diluted by adding $100 \mathrm{~mL}$ hexane and filtered with a Buchner funnel with a sintered glass disc and the filtrate was concentrated on a rotary evaporator, and the residue was subjected to silica column chromatography using hexane as eluent. Compound 1 of $9.0 \mathrm{~g}$ was isolated as a colorless oil in a yield of $90 \%$ and stored under nitrogen environment.

${ }^{1} \mathrm{H}$ NMR (300 MHz, $\left.\mathrm{CDCl}_{3}\right) \delta$ (ppm): 9.47 (d, 1H), 1.56 (br, m, 1H), 1.29-1.13 (m, 32H), 0.81-0.75 (t, 6H).

\section{2-Octyl-1-(thiophen-3-yl)dodecanol (2)}

To $150 \mathrm{~mL}$ round-bottom flask, 1.3M isopropyl magnesium chloride-Lithium chloride complex ( $27 \mathrm{~mL}, 35.1 \mathrm{mmol}, 1.06$ equiv) was added and cooled to $0^{\circ} \mathrm{C}$ then, 
3-bromothiophene (6.5 g, $39.73 \mathrm{mmol}, 1.2$ equiv) was added dropwise and stirred for $30 \mathrm{~min}$ at that temperature followed by warming to room temperature and left under stirring for $24 \mathrm{~h}$, at this point the colour of the reaction mixture becomes yellow. After cooling back to $0^{\circ} \mathrm{C}$, compound $\mathbf{1}(7.8 \mathrm{~g}, 33.11 \mathrm{mmol}, 1$ equiv) was added dropwise. The final mixture was allowed to warm to room temperature and was stirred for $12 \mathrm{~h}$. This reaction then was quenched with $50 \mathrm{~mL}$ water and extracted with ethyl acetate. The combined extracts were dried over anhydrous $\mathrm{MgSO}_{4}$, filtered and concentrated on a rotary evaporator, yields $11 \mathrm{~g}$ of crude product which was then directly used to the next step without further purification.

\section{3-(2-octyldodecyl)thiophene (3) ${ }^{197}$}

To $150 \mathrm{~mL}$ round-bottom flask containing $75 \mathrm{~mL}$ anhydrous diethyl ether and cooled to $0{ }^{\circ} \mathrm{C}, \mathrm{LiAlH}_{4}\left(3.29 \mathrm{~g}, 86.69 \mathrm{mmol}, 3\right.$ equiv) and $\mathrm{AlCl}_{3}(5.78 \mathrm{~g}, 43.335$ mmol, 1.5 equiv) were added in portion respectively under nitrogen stream, after stirring for a few minutes crude mixture of compound 2 (11 g, 28.89 mmol, 1 equiv) was added dropwise to the mixture, and then stirred at room temperature overnight. This reaction was quenched with water $(100 \mathrm{~mL})$ and then $50 \mathrm{~mL}$ concentrated hydrochloric acid was added. The organic phase was separated and extracted with ethyl acetate $(3 \times 50 \mathrm{~mL})$. The combined extracts were dried over anhydrous $\mathrm{MgSO}_{4}$, filtered and concentrated on a rotary evaporator, which was flash chromatographed on silica using hexane as eluent. Compound $\mathbf{3}$ of $4.873 \mathrm{~g}$ was isolated as a colorless oil in a yield of $50.26 \%$ from two steps. 
${ }^{1} \mathrm{H}$ NMR $\left(300 \mathrm{MHz}, \mathrm{CDCl}_{3}\right) \delta(\mathrm{ppm}):$ 7.28-7.24 (m, 1H), 6.94-6.91(m, 2H), 2.61-2.59 (d, 2H), 1.79-1.75 (m, 1H), 1.19-1.18 (br, 32H), 0.95-0.88 (t, 6H).

\section{2-bromo-3-(2-octyldodecyl)thiophene (4)}

To $150 \mathrm{~mL}$ round-bottom flask, containing compound $8(1 \mathrm{~g}, 2.742 \mathrm{mmol}, 1$ equiv) and $60 \mathrm{~mL}$ dimethylformamide (DMF), N-bromosuccinimide (NBS) $(0.5 \mathrm{~g}$, $2.809 \mathrm{mmol}, 1.02$ equiv) was added in portions at $0{ }^{\circ} \mathrm{C}$, then the mixture was stirred overnight at room temperature. Next, the reaction mixture was concentrated on a rotary evaporator, and then it was washed with water $(3 \times 50 \mathrm{~mL})$ and brine $(2 \times 30$ $\mathrm{mL}$ ). The combined extracts were dried over anhydrous $\mathrm{MgSO}_{4}$, filtered and concentrated on a rotary evaporator, which was flash chromatographed on silica using hexane as eluent. Compound $\mathbf{4}$ of $0.98 \mathrm{~g}$ was isolated as a colorless oil in a yield of $80.99 \%$.

${ }^{1} \mathrm{H}$ NMR (300 MHz, $\left.\mathrm{CDCl}_{3}\right) \delta(\mathrm{ppm}): 7.25(\mathrm{~d}, 1 \mathrm{H}), 6.85(\mathrm{~d}, 1 \mathrm{H}), 2.61(\mathrm{~d}, 2 \mathrm{H})$, $1.75(\mathrm{~m}, 1 \mathrm{H}), 1.28-1.45(\mathrm{br}, 32 \mathrm{H}), 0.95-0.88(\mathrm{t}, 6 \mathrm{H})$.

\section{Synthesis of trimethyl(4-(2-octyldodecyl)thiophen-2-yl)stannane (5)}

Compound 3 (2.12 g, $8.24 \mathrm{mmol})$ was dissolved in THF $(30 \mathrm{~mL})$ and cooled at $-78{ }^{\circ} \mathrm{C} . n$ - $\mathrm{BuLi}$ (7.02 mmol, 1.5 $\mathrm{M}$ in hexanes) was added dropwise and stirred for $30 \mathrm{~min}$. The reaction mixture was warmed to room temperature and stirred for 30 min then cooled at $-78{ }^{\circ} \mathrm{C}$ again followed by the dropwise addition of trimethyltin chloride (7.02 $\mathrm{mL}, 7.02 \mathrm{mmol}, 1 \mathrm{M}$ in THF). Subsequently, the reaction mixture was warmed to room temperature and stirred for overnight. Water $(20 \mathrm{~mL})$ was added to 
the mixture and extracted with diethyl ether $(30 \mathrm{~mL} \times 2)$. The combined organic layer was dried over $\mathrm{MgSO}_{4}$ and concentrated under reduced pressure to give crude organotin, compound $5(2.97 \mathrm{~g}, 96 \%)$ as a brown liquid. Organotin (5) was used directly in next reaction without further purification.

${ }^{1} \mathrm{H}$ NMR $\left(300 \mathrm{MHz}, \mathrm{CDCl}_{3}\right) \delta 0.40(\mathrm{~s}, 9 \mathrm{H}), 0.88(\mathrm{t}, 6 \mathrm{H}, J=6.4 \mathrm{~Hz}), 1.20-1.24$ (m, 32H), 1.51-1.53 (m, 1H), 2.21 (d, 2H, $J=6.4 \mathrm{~Hz}), 6.98(\mathrm{~s}, 1 \mathrm{H}), 7.12(\mathrm{~s}, 1 \mathrm{H})$.

\section{2,5-bis(3-(2-octyldodecyl)thiophen-2-yl)thieno[3,2-b]thiophene (6) ${ }^{197}$}

Compound 4 (0.952 g, $2.147 \mathrm{mmol}, 2$ equiv), 2,bis(trimethylstannyl) thieno[3,2-b]thiophene (0.5 g, $1.073 \mathrm{mmol}, 1$ equiv), $\mathrm{Pd}_{2}\left(\mathrm{dba}_{3}(0.05 \mathrm{~g}, 0.055 \mathrm{mmol}\right.$, 0.05 equiv) and $\mathrm{P}(\mathrm{o} \text {-tolyl })_{3}(0.12 \mathrm{~g}, 0.4 \mathrm{mmol}, 0.37$ equiv) were added in $50 \mathrm{~mL}$ round-bottom flask under nitrogen stream and then placed under vacuum for about 15 min followed by degassing via nitrogen bubbling for another $15 \mathrm{~min}$ and then anhydrous THF $(10 \mathrm{~mL})$ were added under nitrogen stream. After being stirred for $12 \mathrm{~h}$ under reflux, the reaction was quenched with $20 \mathrm{~mL}$ of water and the $\mathrm{pH}=1$ was adjusted by adding concentrated hydrochloric acid, extracted with ethyl acetate $3 \times 20 \mathrm{~mL}$. The combined extracts were dried over anhydrous $\mathrm{Na}_{2} \mathrm{SO}_{4}$, filtered and concentrated on a rotary evaporator, which was flash chromatographed on silica using hexane as eluent. Compound $\mathbf{6}$ of $0.31 \mathrm{~g}$ was isolated as a yellow-brown sticky oil in a yield of $44.37 \%$.

${ }^{1} \mathrm{H}$ NMR $\left(300 \mathrm{MHz}, \mathrm{CDCl}_{3}\right) \delta(\mathrm{ppm}):$ 7.25-7.24 (d, 4H), $7.19(\mathrm{~s}, 2 \mathrm{H}), 2.76(\mathrm{~d}$, 4H), $1.59(\mathrm{~m}, 2 \mathrm{H}), 1.38-1.18(\mathrm{br}, 64 \mathrm{H}), 0.95-0.88(\mathrm{t}, 12 \mathrm{H})$. 


\section{3, 3"',-bis(2-octyldodecyl)-2,2':5',2":5",2'"-quaterthiophene (7)}

It was synthesized as yellow oil in a yield of $41.7 \%$ following the similar method of compound 6 .

${ }^{1} \mathrm{H}$ NMR (300 MHz, CDCl $) \delta(\mathrm{ppm}): 7.07$ (d, 2H), 7.01 (d, 2H), $6.95(\mathrm{~d}, 2 \mathrm{H})$, $6.8(\mathrm{~d}, 2 \mathrm{H}), 2.65(\mathrm{~d}, 4 \mathrm{H}), 1.6(\mathrm{~m}, 2 \mathrm{H}), 1.05-1.27(\mathrm{br}, 64 \mathrm{H}), 0.74-0.77(\mathrm{t}, 12 \mathrm{H})$.

\section{2,5-bis(3-(2-octyldodecyl)-5-(trimethylstannyl)thiophen-2-yl)thieno[3,2b]}

thiophene (8)

To a solution of compound 6 ( $0.28 \mathrm{~g}, 0.32 \mathrm{mmol}, 1$ equiv) in THF $(20 \mathrm{~mL})$, at $-78{ }^{\circ} \mathrm{C}$ was added $n$ - BuLi ( $0.323 \mathrm{~mL}, 0.7117 \mathrm{mmol}, 2.2 \mathrm{M}$ in hexanes) dropwise, the resulting suspension was warmed naturally to $0{ }^{\circ} \mathrm{C}$ and then stirred for $1 \mathrm{~h}$, cooled back to $-78{ }^{\circ} \mathrm{C}$ and $\mathrm{Me}_{3} \mathrm{SnCl}(0.744 \mathrm{~mL}, 0.744 \mathrm{mmol}, 2.3$ equiv $)$ was added dropwise, the solution was then allowed to warm to room temperature and stir for $16 \mathrm{~h}$. Then, it was poured $30 \mathrm{~mL}$ of water and extracted with hexane $(3 \times 20 \mathrm{~mL})$, the combined extracts were washed with water $(5 \times 30 \mathrm{~mL})$, brine $(2 \times 20 \mathrm{~mL})$, and then dried over anhydrous $\mathrm{Na}_{2} \mathrm{SO}_{4}$, filtered and concentrated on rotary evaporator to give $\mathbf{8}(0.34 \mathrm{~g}$, $88.3 \%)$ as a yellow-brown oil.

${ }^{1} \mathrm{H}$ NMR (300 MHz, $\left.\mathrm{CDCl}_{3}\right) \delta(\mathrm{ppm}): 7.09$ (s, 2H), 6.83 (s, 2H), $2.6(\mathrm{~d}, 4 \mathrm{H})$, $1.7(\mathrm{~m}, 2 \mathrm{H}), 1.33-1.18(\mathrm{br}, 64 \mathrm{H}), 0.69-0.80(\mathrm{t}, 12 \mathrm{H}), 0.25(\mathrm{~s}, 18 \mathrm{H})$. 


\section{$(3,3$ '"-bis(2-octyldodecyl)-[2,2':5',2':5",2'"-quaterthiophene]-5,5"'diyl)bis(tri}

methylstannane) (9)

It was synthesized as yellow oil in a yield of $70.88 \%$ following the similar method of compound $\mathbf{8}$.

${ }^{1} \mathrm{H}$ NMR (300 MHz, $\left.\mathrm{CDCl}_{3}\right) \delta(\mathrm{ppm}): 7.01(\mathrm{~d}, 2 \mathrm{H}), 6.90(\mathrm{~d}, 2 \mathrm{H}), 6.87(\mathrm{~s}, 2 \mathrm{H})$, $2.65(\mathrm{~d}, 4 \mathrm{H}), 1.7(\mathrm{~m}, 2 \mathrm{H}), 1.33-1.18(\mathrm{br}, 64 \mathrm{H}), 0.69-0.80(\mathrm{t}, 12 \mathrm{H}), 0.25(\mathrm{~s}, 18 \mathrm{H})$.

\section{1,2-di(thiophen-3-yl)ethane-1,2-dione (10) ${ }^{198}$}

A solution of 3-lithiumthiophene, labeled as Solution A, was prepared as follows: $44 \mathrm{~mL}$ of $1.6 \mathrm{M} \mathrm{n}$-butyllithium (1.2 equiv., $60 \mathrm{mmol}$ ) in hexanes was added via cannula to $100 \mathrm{~mL}$ of anhydrous $\mathrm{THF}$, previously cooled to $-78^{\circ} \mathrm{C}$ (Acetone + liquid $\mathrm{N}_{2}$ ). The mixture was stirred for 10 minutes, and then 3-bromothiophene $(8.125 \mathrm{~g}, 50 \mathrm{mmol})$ was added dropwise. The mixture was stirred for $\sim 150$ minutes, keeping the temperature at $-78{ }^{\circ} \mathrm{C}$. Meanwhile, a solution, labelled as Solution B, was prepared as follows: In a $500 \mathrm{~mL}$ round bottom flask (equipped with stir bar and a septum), containing $200 \mathrm{~mL}$ of anhydrous THF, was added $\mathrm{LiBr}(5.211 \mathrm{~g}, 60 \mathrm{mmol}$, 1.2 equiv.) and $\mathrm{CuBr}$ (8.607 g, $60 \mathrm{mmol}, 1.2$ equiv), the $\mathrm{CuBr}$ and $\mathrm{LiBr}$ mixture was stirred until all the salts dissolved, then this mixture was cooled to $-40{ }^{\circ} \mathrm{C}$ or to lower temperatures. Solution C: oxalyl chloride ( $3.173 \mathrm{~g}, 25 \mathrm{mmol}$, 0.5 equiv) was dissolved in $10 \mathrm{~mL}$ of anhydrous THF in a $50-\mathrm{mL}$ round bottom flask (previously equipped with a septum) and cooled to $-40{ }^{\circ} \mathrm{C}$ or to lower temperatures. Solution A was added via cannula to Solution B, and the mixture was strongly stirred for $\sim 5$ 
minutes; then the Solution $\mathrm{C}$ was slowly added via cannula. The mixture was kept in the cold bath for $2 \mathrm{~h}$, allowed to warm up to room temperature and left for overnight under stirring in $\mathrm{N}_{2}$ and quenched with $100 \mathrm{~mL}$ of saturated $\mathrm{NH}_{4} \mathrm{Cl}(\mathrm{aq})$. It was transferred to separatory funnel and the aqueous phase was separated from the organic phase and extracted with ethyl acetate $(20 \mathrm{~mL} 3 \times)$, then all the organic phase were combined and washed with water $(30 \mathrm{~mL}, 2 \times)$, brine solution $(30 \mathrm{~mL}, 2 \times)$, the organic mixture was placed in $\mathrm{MgSO}_{4}$ for drying. Concentrated in a rotary evaporator and then purified by column chromatography (silica, and 1:3 of ethyl acetate: hexanes). An orange-yellow solid was isolated, $2.54 \mathrm{~g}, 78 \%$ yield.

${ }^{1} \mathrm{H}$ NMR $\left(300 \mathrm{MHz}, \mathrm{CDCl}_{3}\right): \delta(\mathrm{ppm}): 8.35-8.34(\mathrm{~m}, 2 \mathrm{H}), 7.70-7.68(\mathrm{~m}, 2 \mathrm{H})$, $7.40-7.32(\mathrm{~m}, 2 \mathrm{H})$

\section{Benzo[1,2-b:6,5-b']dithiophene-4,5-dione (BDTD) (11) ${ }^{198}$}

To a 250-mL round bottom flask, equipped with a stir bar and an inlet adapter and containing $100 \mathrm{~mL}$ of DCM, was added anhydrous $\mathrm{FeCl}_{3}(11.843 \mathrm{~g}, 73.017$ mmol, 3 equiv). The mixture was stirred for a few seconds, and then the diketone $\mathbf{1 0}$ (5.41 g, 24.339 mmol, 1 equiv) was added in one portion under a nitrogen stream, and then the reaction mixture was stirred for overnight at room temperature. The mixture was quenched with $\sim 100 \mathrm{~mL}$ of chilled water. Afterwards, It was transferred to separatory funnel and the aqueous phase was separated from the organic phase and extracted with DCM $(20 \mathrm{~mL}, 3 \times)$, then all the organic phase were combined and washed with water $(30 \mathrm{~mL}, 2 \times)$, brine solution $(30 \mathrm{~mL}, 2 \times)$; the organic mixture was 
placed in $\mathrm{MgSO}_{4}$ for drying. After filtration, it was concentrated in a rotary evaporator and then purified by column chromatography (silica, and dichloromethane). A black solid was isolated, $4.43 \mathrm{~g}, 82.65 \%$ yield.

${ }^{1} \mathrm{H}$ NMR (300 MHz, $\left.\mathrm{CDCl}_{3}\right): \delta(\mathrm{ppm}): 7.51(\mathrm{~d}, 2 \mathrm{H}), 7.27(\mathrm{~d}, 2 \mathrm{H})$.

\section{Benzo[1,2-b:6,5-b']dithiophene-4,5-diamine (BDTDA) (12) ${ }^{198}$}

To a $250 \mathrm{~mL}$ three-neck round-bottom flask, containing a stir bar, and under a nitrogen atmosphere, was added BDTD (4.43 g, $20.11 \mathrm{mmol}, 1$ equiv), hydroxylamine hydrochloride ( $3.49 \mathrm{~g}, 50.275 \mathrm{mmol}, 2.5$ equiv), and $100 \mathrm{~mL}$ of anhydrous ethanol. The flask was equipped with a condenser and the mixture was warmed to reflux $\left(75-80{ }^{\circ} \mathrm{C}\right)$ and stirred for $24 \mathrm{~h}$. The reaction mixture was cooled to room temperature, and $0.443 \mathrm{~g}$ of $10 \%$ of $\mathrm{Pd}$ on activated carbon $(\mathrm{Pd} / \mathrm{C})$ was added. An addition funnel containing a solution of hydrazine hydrate $(10 \mathrm{~mL}$ of $\mathrm{N}_{2} \mathrm{H}_{4} \cdot \mathrm{xH}_{2} \mathrm{O}$ in $20 \mathrm{~mL}$ of $\mathrm{EtOH}$ ) was placed on one the side arm of the round-bottom flask. The reaction mixture was warmed up to $65^{\circ} \mathrm{C}$, and then the hydrazine solution was added dropwise for $\sim 30 \mathrm{~min}$. The reaction temperature was increased to $85^{\circ} \mathrm{C}$, and then the mixture was stirred for $48 \mathrm{~h}$. The mixture was allowed to cool to $\sim 60^{\circ} \mathrm{C}$ and filtered (by gravity filtration, and the filter was washed with ethanol to recover the entire product). The solvent was removed by rotary evaporation, and the resulting solid was dispersed in water, filtered, washed with plenty water and cold ethanol it is collected after drying under vacuum to give $1.51 \mathrm{~g}, 34 \%$ yield. 
${ }^{1} \mathrm{H}$ NMR (300 MHz, $\left.\mathrm{CDCl}_{3}\right): \delta(\mathrm{ppm}): 7.5(\mathrm{~d}, 2 \mathrm{H}) 7.35(\mathrm{~d}, 4 \mathrm{H}), 3.85-3.52(\mathrm{~s}$, br, $4 \mathrm{H})$.

\section{Dithieno[3' $\left.2^{\prime}: 3,4 ; 2^{\prime \prime}, 3^{\prime \prime}: 5,6\right]$ benzo[1,2-c][1,2,5]thiadiazole (DT-BTD) (13a) ${ }^{199}$}

A mixture of diamine compound 12 (0.7 g, 3.177 mmol, 1 equiv), $\mathrm{CH}_{2} \mathrm{Cl}_{2}$ (10 $\mathrm{mL}$ ), and $\mathrm{Et}_{3} \mathrm{~N}$ (1.286 g, $12.708 \mathrm{mmol}, 4$ equiv) was stirred until the diamine compound 12 was dissolved. After the dropwise addition of 1M thionyl chloride (7.94 mL, 7.94 mmol, 2.5 equiv) in THF, the mixture was refluxed for $5 \mathrm{~h}$. Then, it was quenched by adding $10 \mathrm{~mL}$ of $\mathrm{H}_{2} \mathrm{O}$ and stirred for $30 \mathrm{~min}$. After $\mathrm{pH}$ was adjusted to 2 with concentrated $\mathrm{HCl}$, the mixture was extracted with $\mathrm{CH}_{2} \mathrm{Cl}_{2}(3 \times 20 \mathrm{~mL})$. The organic phase was combined, washed with $\mathrm{H}_{2} \mathrm{O}(2 \times 30 \mathrm{~mL})$, and dried over $\mathrm{MgSO}_{4}$. After filtration, the filtrate was concentrated, and the residue was subjected to silica column chromatography using petroleum $\mathrm{CH}_{2} \mathrm{Cl}_{2}$ as eluent, $0.623 \mathrm{~g}$ of DT-BTD as a yellow solid in a yield of $78.95 \%$.

${ }^{1} \mathrm{HNMR}\left(300 \mathrm{MHz}, \mathrm{CDCl}_{3}\right) \delta(\mathrm{ppm}): 8.05(\mathrm{~d}, 2 \mathrm{H}), 7.56(\mathrm{~d}, 2 \mathrm{H})$.

\section{Dithieno[3',2':3,4;2',3':5,6]benzo[1,2-c]oxadiazole (DT-BOD) (13b)}

To a $150 \mathrm{~mL}$ glass pressure vessel, equipped with a stir bar, was added the diketone BDTD $(0.936 \mathrm{~g}, 4.25 \mathrm{mmol}, 1$ equiv), hydroxylamine hydrochloride (0.738g, $10.62 \mathrm{mmol}, 2.5$ equiv), and $70 \mathrm{~mL}$ of anhydrous ethanol. The vessel was equipped with its respective Teflon cap, and the mixture was stirred at $85^{\circ} \mathrm{C}$ for 24 h. The temperature was then increased to $140{ }^{\circ} \mathrm{C}$, and the reaction mixture was stirred for $60 \mathrm{~h}$. The mixture was cooled to room temperature and transferred to a round 
bottom flask. The solvent was removed by rotary evaporation, and the resulting solid was washed with $\mathrm{H}_{2} \mathrm{O}(2 \times 30 \mathrm{~mL})$, and dried over $\mathrm{MgSO}_{4}$. After filtration, the filtrate was concentrated, and the residue was subjected to silica column chromatography using Hexane: $\mathrm{CH}_{2} \mathrm{Cl}_{2}(1: 1, \mathrm{v} / \mathrm{v})$ as eluent. A yellow solid, DT-BOD was recovered, $0.3 \mathrm{~g}, 30 \%$ yield.

${ }^{1} \mathrm{H}$ NMR (300 MHz, $\left.\mathrm{CDCl}_{3}\right) \delta(\mathrm{ppm}): 7.94(\mathrm{~d}, 2 \mathrm{H}), 7.58(\mathrm{~d}, 2 \mathrm{H})$.

\section{5,8-dibromodithieno[3',2':3,4;2',,3':5,6]benzo[1,2-c][1,2,5]thiadiazole (Br2-DT -BTD) (14a)}

To a $250 \mathrm{~mL}$ round-bottom flask, equipped with a stir bar and a condenser was added DT-BTD (0.623 g, $2.5 \mathrm{mmol}, 1$ equiv), chloroform (200 mL), and bromine

(0.282 mL, $0.8819 \mathrm{~g}, 5.519 \mathrm{mmol}, 2.2$ equiv.). The reaction mixture was warmed to $80-85^{\circ} \mathrm{C}$ and stirred for $24 \mathrm{~h}$. The reaction mixture was cooled to room temperature, and the resulting solid was filtered, washed with chloroform, air-dried, and put under vacuum to remove solvent traces. A bright yellow solid was recovered, $0.76 \mathrm{~g}, 75 \%$ yield.

${ }^{1} \mathrm{H}$ NMR (500 MHz, hot-chlorobenzene- $d$ ): $\delta(\mathrm{ppm}): 7.95$ (s, 2H) Anal. Calcd for $\mathrm{C}_{10} \mathrm{H}_{2} \mathrm{~N}_{2} \mathrm{~S}_{3} \mathrm{Br}_{2}$ : C, 29.59; H, 0.5; N, 6.90; S, 23.63; Br, 39.38. Found: C, 29.86; H, 1.25; N, 6.93; S, 24.06; Br, 37.89

\section{5,8-dibromodithieno[3',2':3,4;2',3':5,6]benzo[1,2-c]oxadiazole (14b)}

The reaction was carried using the same procedure as 14a. The workup has been modified as follows: after $12 \mathrm{~h}$ of reaction, it was allowed to cool to room 
temperature while cooling the mixture was diluted with an aqueous $\mathrm{Na}_{2} \mathrm{~S}_{2} \mathrm{O}_{3}$ solution. Subsequently, the mixture was separated and washed with saturated aqueous $\mathrm{NaHCO}_{3}$ solution and brine. Then dried over $\mathrm{MgSO}_{4}$, filtered and concentrated under vacuum, and the solid product was recrystallized from odichlorobenzene to give $\mathbf{1 4 b}$ as a yellow crystalline solid (69\% yield).

${ }^{1} \mathrm{H}$ NMR (300 MHz, $\left.\mathrm{CDCl}_{3}\right) \delta(\mathrm{ppm}): 7.92(\mathrm{~s}, 2 \mathrm{H})$.

\section{5,8-bis(4-(2-octyldodecyl)thiophen-2-yl)dithieno[3',2':3,4;2' ',3":5,6]benzo [1,2-} c] $[1,2,5]$ thiadiazole $(15 \mathbf{a})$

Compound 14a (0.2 g, $0.492 \mathrm{mmol})$ and Compound 5 (0.675 g, $1.28 \mathrm{mmol})$ were added to $40 \mathrm{~mL}$ toluene in a $100 \mathrm{~mL}$ round-bottom flask and purged with $\mathrm{N}_{2}$ for 30min. $\mathrm{Pd}\left(\mathrm{PPh}_{3}\right)_{4}(29 \mathrm{mg}, 0.025 \mathrm{mmol})$ was added to the mixture and refluxed for $48 \mathrm{~h}$. After the mixture was cooled to room temperature, the solvent was evaporated under reduced pressure, and then dissolved in $50 \mathrm{~mL}$ diethyl ether and extensively washed with water and brine. The organic phase was separated and dried over anhydrous $\mathrm{MgSO}_{4}$. After removal of the solvent by vacuum evaporation, the residue was purified by column chromatography on silica gel $\left(\mathrm{CH}_{2} \mathrm{Cl}_{2} /\right.$ hexane = 1:10) to give Compound 15a as a yellow oil (0.34 g, 71\%).

${ }^{1} \mathrm{H}$ NMR (300 MHz, CDCl3) $\delta(\mathrm{ppm}): 8.00(\mathrm{~d}, 2 \mathrm{H}), 7.15$ (s, 2H), $6.90(\mathrm{~s}, 2 \mathrm{H})$, $2.57(\mathrm{~d}, 4 \mathrm{H}), 1.65(\mathrm{br}, 2 \mathrm{H}), 1.38-1.13(\mathrm{~m}, 64 \mathrm{H}), 0.92-0.81(\mathrm{~m}, 12 \mathrm{H})$. 


\section{5,8-bis(4-(2-octyldodecyl)thiophen-2-yl)dithieno[3',2':3,4; $2^{\prime \prime}, 3^{\prime \prime}:$ 5,6]benzo}

\section{$[1,2 c]-[1,2,5]$ oxadiazole $(15 b)$}

Compound 15b was synthesized in the same procedure with Compound 15a. Yield: $84 \%$.

${ }^{1} \mathrm{H}$ NMR (300 MHz, $\left.\mathrm{CDCl}_{3}\right) \delta(\mathrm{ppm}): 7.90(\mathrm{~d}, 2 \mathrm{H}), 7.15(\mathrm{~d}, 2 \mathrm{H}), 6.92(\mathrm{~d}, 2 \mathrm{H})$, $2.55(\mathrm{~d}, 4 \mathrm{H}), 1.64(\mathrm{br}, 2 \mathrm{H}), 1.38-1.13(\mathrm{~m}, 64 \mathrm{H}), 0.92-0.80(\mathrm{~m}, 12 \mathrm{H})$.

\section{5,8-bis(5-bromo-4-(2-octyl dodecyl)thiophen-2-yl)dithieno[3',2' :3,4;2",3":}

\section{$5,6]$-benzo[1,2-c][1,2,5]thiadiazole (16a)}

N-Bromosuccinimide $(0.137 \mathrm{~g}, 0.768 \mathrm{mmol})$ was added portion-wise to a solution of Compound 15a (0.34 g, $0.349 \mathrm{mmol})$ in $80 \mathrm{~mL}$ of THF at room temperature. After the mixture was stirred for $12 \mathrm{~h}$, the $\mathrm{NaHCO}_{3}$ solution was added, and the mixture was extracted with $\mathrm{CH}_{2} \mathrm{Cl}_{2}$. The organic layer was washed with water and brine and then dried over anhydrous $\mathrm{MgSO}_{4}$. The solvent was removed by vacuum evaporation, and the residue was purified by column chromatography on silica gel $\left(\mathrm{CH}_{2} \mathrm{Cl}_{2} /\right.$ hexane $\left.=1: 10\right)$ to give Compound 16a as a yellow oil $(0.277 \mathrm{~g}$, $70 \%)$.

${ }^{1} \mathrm{H}$ NMR (300 MHz, $\left.\mathrm{CDCl}_{3}\right) \delta(\mathrm{ppm}): 7.92(\mathrm{~s}, 2 \mathrm{H}), 6.99(\mathrm{~s}, 2 \mathrm{H}), 2.52(\mathrm{~d}, 4 \mathrm{H})$, $1.70(\mathrm{br}, 2 \mathrm{H}), 1.38-1.12(\mathrm{~m}, 64 \mathrm{H}), 0.93-0.78(\mathrm{~m}, 12 \mathrm{H})$. 


\section{5,8-bis(5-bromo-4-(2-octyl dodecyl)thiophen-2-yl)dithieno[3',2': 3,4; 2",3":}

\section{$5,6]$-benzo[1,2-c][1,2,5]oxadiazole (16b)}

Compound 16b was synthesized in the same procedure with Compound 16a. Yield: 92\%.

${ }^{1} \mathrm{H}$ NMR (300 MHz, $\left.\mathrm{CDCl}_{3}\right) \delta(\mathrm{ppm}): 7.80(\mathrm{~s}, 2 \mathrm{H}), 6.97$ (s, 2H), $2.52(\mathrm{~d}, 4 \mathrm{H})$, $1.69(\mathrm{br}, 2 \mathrm{H}), 1.41-1.02(\mathrm{~m}, 64 \mathrm{H}), 0.94-0.76(\mathrm{~m}, 12 \mathrm{H})$.

\subsubsection{Synthesis of polymer P1, P2, P3, PF-S, PF-O}

\subsubsection{Previous synthesis of $P 1$ and $P 2$}

\section{Poly[(dithieno[(3',2':3,4;2",3'":5,6]benzo[1,2-c][1,2,5]thiadiazole-5,8-diyl)-alt-}

\section{(2,5-bis(2- octyldodecyl)thiophen-2-yl)thieno[3,2-b]thiophene-5,5'-diyl)] (P1)}

To a $25 \mathrm{~mL}$ round-bottom flask dibromide monomer (compound 14a) $(0.1$ mmol), bis(trimethylstannyl)-substituted monomer (8) $(0.1 \mathrm{mmol})$, were added and placed under vacuum for about $15 \mathrm{~min}$ and followed by adding $\mathrm{Pd}\left(\mathrm{PPh}_{3}\right)_{4}(1.1 \mathrm{mg}$, $0.001 \mathrm{mmol}$ ) under nitrogen stream after a few minutes later dry toluene was added and degassed via nitrogen bubbling for about $30 \mathrm{~min}$. It was stirred for 3 days at 110 ${ }^{\circ} \mathrm{C}$ and followed by precipitation in $100 \mathrm{~mL}$ of methanol after being cooled down to room temperature and then filtration. The crude polymer was subjected to Soxhlet extraction with methanol, hexane, and chloroform solvents in sequence. The chloroform fraction was evaporated to dryness and reprecipitated from methanol to 
give the $\mathbf{P l}$ as a dark powder (60 mg, yield $54.5 \%), M_{\mathrm{n}} / \mathrm{PDI}$ of $9.9 \mathrm{kDa} / 8.4 \mathrm{using}$ THF as eluent at R.T.

${ }^{1} \mathrm{H}$ NMR $\left(300 \mathrm{MHz}, \mathrm{CDCl}_{3}\right) \delta(\mathrm{ppm}): 7.53(\mathrm{br}, 2 \mathrm{H}), 6.84$ (br, 4H), 2.49 (d, 4H), 1.57 (br, 2H), 1.33-1.03 (br, 64H), 0.81-0.78 (br, 12H). Anal. Calcd for $\mathrm{C}_{64} \mathrm{H}_{90} \mathrm{~N}_{2} \mathrm{~S}_{7}$ : C, 69.18; H, 8.16; N, 2.52; S, 20.14. Found: C, 66.36; H, 7.82; N, 3.16; S, 20.33. (N.B. For all polymers, the number of protons assigned correspond to only a single repeating unit.)

\section{Poly[(dithieno[(3',2':3,4;2",3' ':5,6]benzo[1,2-c][1,2,5]thiadiazole-5,8-diyl)-alt-}

\section{(3,3'"'-bis(2-octyldodecyl)-2,2':5',2":5',2"'-quaterthiophene-5,5'"-diyl)] (P2)}

It was synthesized by the same procedure as $\mathbf{P 1}$. Compound $\mathbf{1 4 a}(0.1 \mathrm{mmol})$ and $9(0.1 \mathrm{mmol})$ was used. P2 was obtained as a dark powder (30 mg, yield 27\%) $M_{\mathrm{n}} / \mathrm{PDI}$ of $3.7 \mathrm{kDa} / 7.6$ using THF as eluent at R.T.

${ }^{1} \mathrm{H}$ NMR (300 MHz, $\left.\mathrm{CDCl}_{3}\right) \delta(\mathrm{ppm}): 7.53(\mathrm{br}, 2 \mathrm{H}), 6.84(\mathrm{br}, 2 \mathrm{H}), 5.32(\mathrm{br}$, 4H), $2.64(\mathrm{~d}, 4 \mathrm{H}), 1.57(\mathrm{br}, 2 \mathrm{H}), 1.33-1.03(\mathrm{br}, 64 \mathrm{H}), 0.81-0.78$ (br, 12H). Anal. Calcd for $\mathrm{C}_{66} \mathrm{H}_{92} \mathrm{~N}_{2} \mathrm{~S}_{7}: \mathrm{C}, 69.71 ; \mathrm{H}, 8.15 ; \mathrm{N}, 2.46 ; \mathrm{S}, 19.68$. Found: C, 62.00; H, 7.91; N, 3.21; S, 18.49 . 


\subsubsection{Improved synthesis of $P 1$ and $P 2$ and also synthesis of polymer $P 3$, PF-S, PF-O}

\section{General synthesis method of Donor-Acceptor polymers (P1, P2, and P3) via Stille polycondensation}

To a $25 \mathrm{~mL}$ round-bottom flask containing bis(trimethylstannyl)-substituted monomers $(0.1 \mathrm{mmol})$, dibromide monomer (Compound 16 of $0.1 \mathrm{mmol})$, and dry toluene (4 mL) were added. After $\mathrm{N}_{2}$ gas purge for $15 \mathrm{~min}, \mathrm{Pd}\left(\mathrm{PPh}_{3}\right)_{4}(3 \mathrm{~mol} \%)$ was added under a nitrogen stream and degassed via nitrogen bubbling for about $30 \mathrm{~min}$. It was stirred for 3 days at $110{ }^{\circ} \mathrm{C}$ and followed by precipitation in $100 \mathrm{~mL}$ of methanol after being cooled down to room temperature and then filtration. The crude polymer was subjected to Soxhlet extraction with methanol, hexane, and chloroform solvents in sequence. The chloroform fraction was evaporated to dryness and reprecipitated from methanol to give $\mathbf{P 1}, \mathbf{P 2}$, and $\mathbf{P 3}$ as a black powder.

\section{Poly[5-(4-(2-methyldodecyl)-5-(thieno[3,2-b]thiophen-2-yl) thiophen-2-yl)-8-}

(4-(2-octyldo decyl)thiophen-2-yl)dithieno $\left[3^{\prime}, 2^{\prime}: 3,4 ; 2^{\prime \prime}, 3^{\prime \prime}: 5,6\right]$ benzo[1,2-c]

\section{$[1,2,5]$ thiadiazole] $(\mathbf{P 1})$}

Yield: $68 \% . M_{\mathrm{n}} / \mathrm{PDI}$ of $8.0 \mathrm{kDa} / 2.54$ using $\mathrm{CHCl}_{3}$ as eluent at $80{ }^{\circ} \mathrm{C}$. Anal. Calcd for $\mathrm{C}_{64} \mathrm{H}_{90} \mathrm{~N}_{2} \mathrm{~S}_{7}: \mathrm{C}, 69.13 ; \mathrm{H}, 8.16 ; \mathrm{N}, 2.52 ; \mathrm{S}, 20.19$. Found: $\mathrm{C}, 65.36 ; \mathrm{H}$, $7.529 ; \mathrm{N}, 3.077 ; \mathrm{S}, 22.05$. 


\section{Poly[5-(3-(2-methyldodecyl)-[2,2':5',2'--terthiophen]-5-yl)-8-(4-(2-octyldo}

decyl)thiophen-2-yl)-dithieno[3',2':3,4;2"',3':5,6]benzo[1,2c][1,2,5]thia diazole](P2)

Yield: $47 \% . M_{\mathrm{n}} / \mathrm{PDI}$ of $9.1 \mathrm{kDa} / 1.89 \mathrm{using} \mathrm{CHCl}_{3}$ as eluent at $80^{\circ} \mathrm{C}$. Anal.Calcd for $\mathrm{C}_{66} \mathrm{H}_{92} \mathrm{~N}_{2} \mathrm{~S}_{7}$ : C, 69.66; H, 8.15; N, 2.46; S, 19.73. Found: C, 67.55; H, 8.375; N, 2.421; S, 19.77 .

Poly[5-(4-(2-methyldodecyl)-5-(thieno[3,2-b]thiophen-2-yl)thiophen-2-yl)-8-(4(2-octyldodecyl)thiophen-2-yl)dithieno[3',2':3,4;2"',3":5,6]benzo[1,2-c][1,2,5] oxadiazole](P3).

Yield: $41 \% . M_{\mathrm{n}} / \mathrm{PDI}$ of $7.6 \mathrm{kDa} / 1.93$ using $\mathrm{CHCl}_{3}$ as eluent at $80{ }^{\circ} \mathrm{C}$. Anal. Calcd for $\mathrm{C}_{64} \mathrm{H}_{90} \mathrm{~N}_{2} \mathrm{OS}_{6}$ : C, 70.15; H, 8.28; N, 2.56; O, 1.46; S, 17.56. Found: C, 65.96; H, 7.854; N, 2.813; S, 18.33 .

General synthesis method of Donor-Acceptor polymers (PF-O and PF-S) by Suzuki polycondensation

To a $25 \mathrm{~mL}$ round-bottom flask dibromide monomer (Compound 16) $(0.1$ mmol),2,2'-(9-(heptadecan-9-ylidene)-9H-fluorene-2,7-diyl)bis(4,4,5,5tetramethyl-1,3,2-dioxaborolane $(0.1 \mathrm{mmol})$, and dry toluene $(4 \mathrm{~mL})$, and $2 \mathrm{M}$ $\mathrm{K}_{2} \mathrm{CO}_{3}$ aqueous solution $(1 \mathrm{~mL})$ were added. After degassed by $\mathrm{N}_{2}$ for $15 \mathrm{~min}$, $\operatorname{Pd}\left(\mathrm{PPh}_{3}\right)_{4}(\sim 3 \mathrm{~mol} \%)$ was added under a nitrogen stream and degassed via nitrogen bubbling for about $30 \mathrm{~min}$. It was stirred for 3 days at $110{ }^{\circ} \mathrm{C}$ and followed by 
precipitation in $100 \mathrm{~mL}$ of methanol after being cooled down to room temperature and then filtration. The resulting crude product was subjected to Soxhlet extraction with methanol, acetone, hexane, and chloroform solvents in sequence. The chloroform fraction was evaporated to dryness and reprecipitated from methanol to give PF-O as a dark red and PF-S were collected from hexane fraction and reprecipitated in methanol to give an orange-red solid.

Poly[5-(5-(9-(heptadecan-9-ylidene)-9H-fluoren-2-yl)-4-(2-octyldodecyl) thiophen-2-yl)-8-(4-(2-octyldodecyl)thiophen-2-yl)dithieno[3',2':3,4;2"',3': 5,6]benzo[1,2-c][1,2,5]thiadiazole] (PF-S)

Yield: $69 \% . M_{\mathrm{n}} / \mathrm{PDI}$ of $12.6 \mathrm{kDa} / 1.42$ using $\mathrm{CHCl}_{3}$ as eluent at $80{ }^{\circ} \mathrm{C}$. Anal. Calcd for $\mathrm{C}_{88} \mathrm{H}_{128} \mathrm{~N}_{2} \mathrm{~S}_{5}$ : C, 76.91; H, 9.39; N, 2.04; S, 11.67. Found: C, 68.37; H, 9.246; N, 1.871; S, 9.666.

Poly[5-(5-(9-(heptadecan-9-ylidene)-9H-fluoren-2-yl)-4-(2-octyldodecyl) thiophen-2-yl)-8-(4-(2-octyldodecyl)thiophen-2-yl)dithieno[3',2':3,4;2"',3':5,6] benzo[1,2-c][1,2,5]oxadiazole] (PF-O)

Yield: $82 \% . M_{\mathrm{n}} / \mathrm{PDI}$ of $5.4 \mathrm{kDa} / 1.59$ using $\mathrm{CHCl}_{3}$ as eluent at $80{ }^{\circ} \mathrm{C}$. Anal. Calcd for $\mathrm{C}_{88} \mathrm{H}_{128} \mathrm{~N}_{2} \mathrm{OS}$ 4: C, 77.82; H, 9.50; N, 2.06; O, 1.18; S, 9.44. Found: C, 76.48; H, 10.05; N, 2.172; S, 9.302. 


\subsubsection{GPC traces for polymers and NMR spectra}

\subsubsection{GPC traces for polymers}
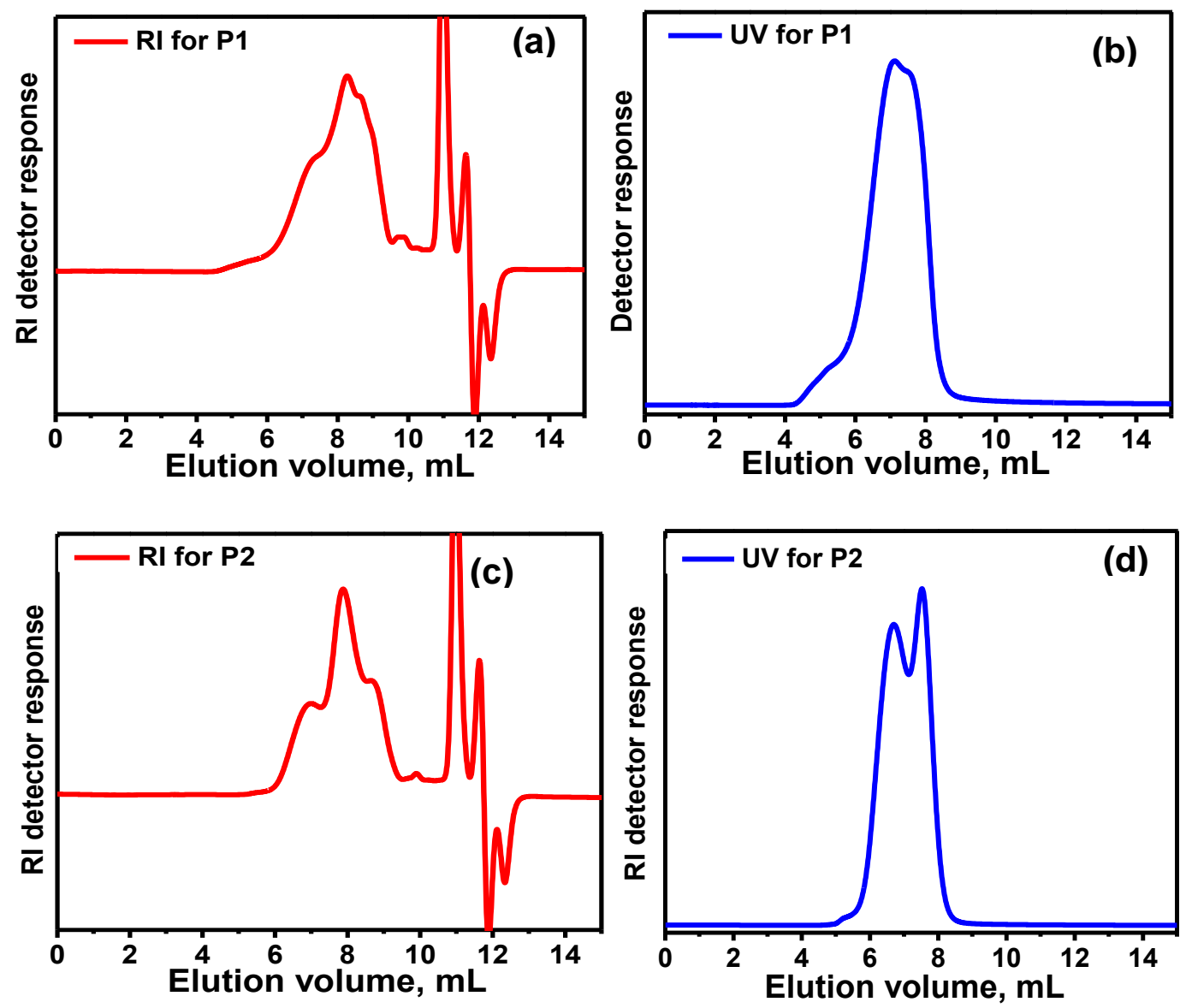

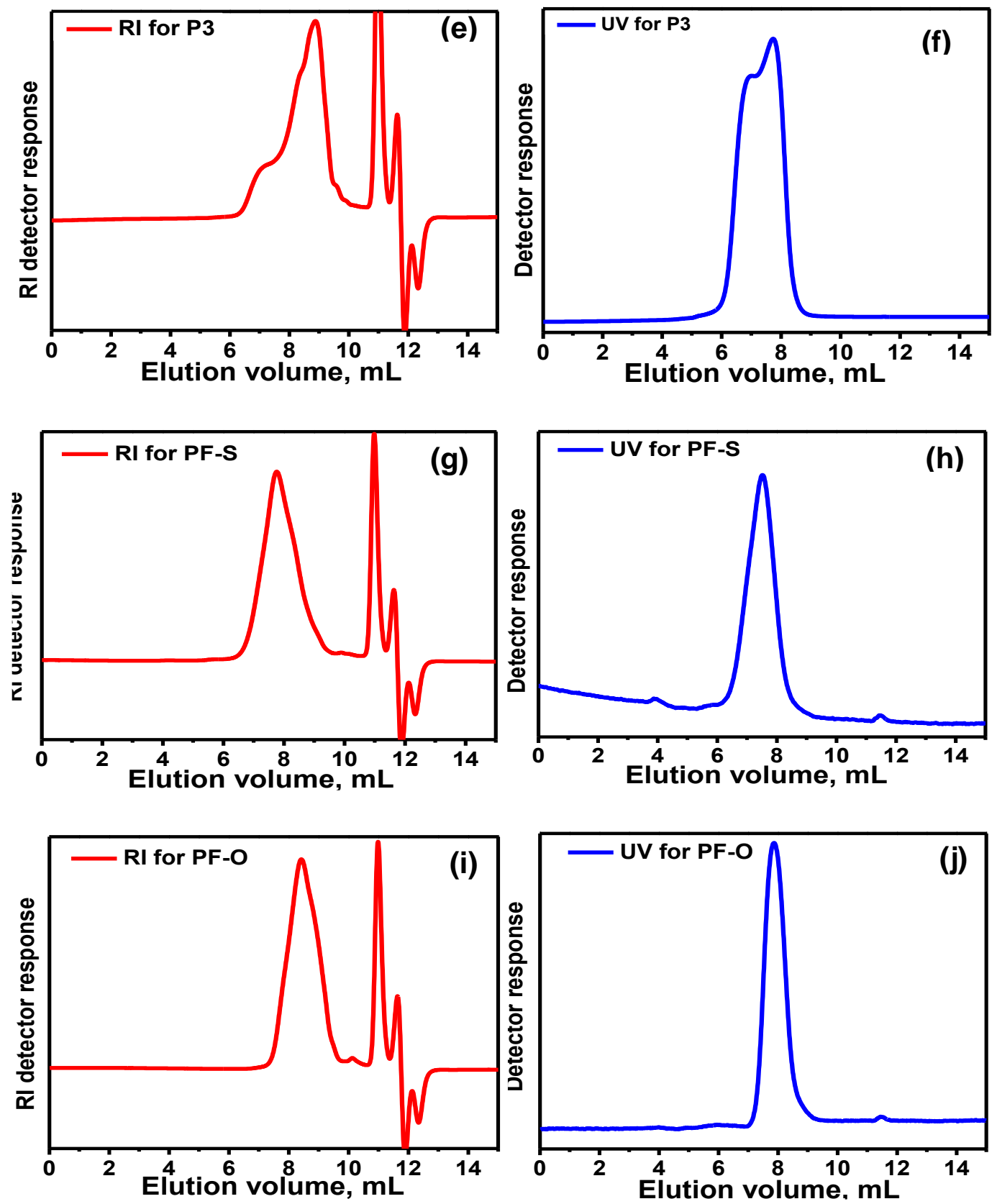

Figure 2.1. GPC traces for P1, P2, P3, PF-S and PF-O (using $\mathrm{CHCl}_{3}$ as eluent at 80

$\left.{ }^{\circ} \mathrm{C}\right)$ from RI (a, c, e, g, i) and UV (550 nm) (b, d, f, h, j) detectors, respectively. 


\subsubsection{NMR spectra of monomers and polymers}


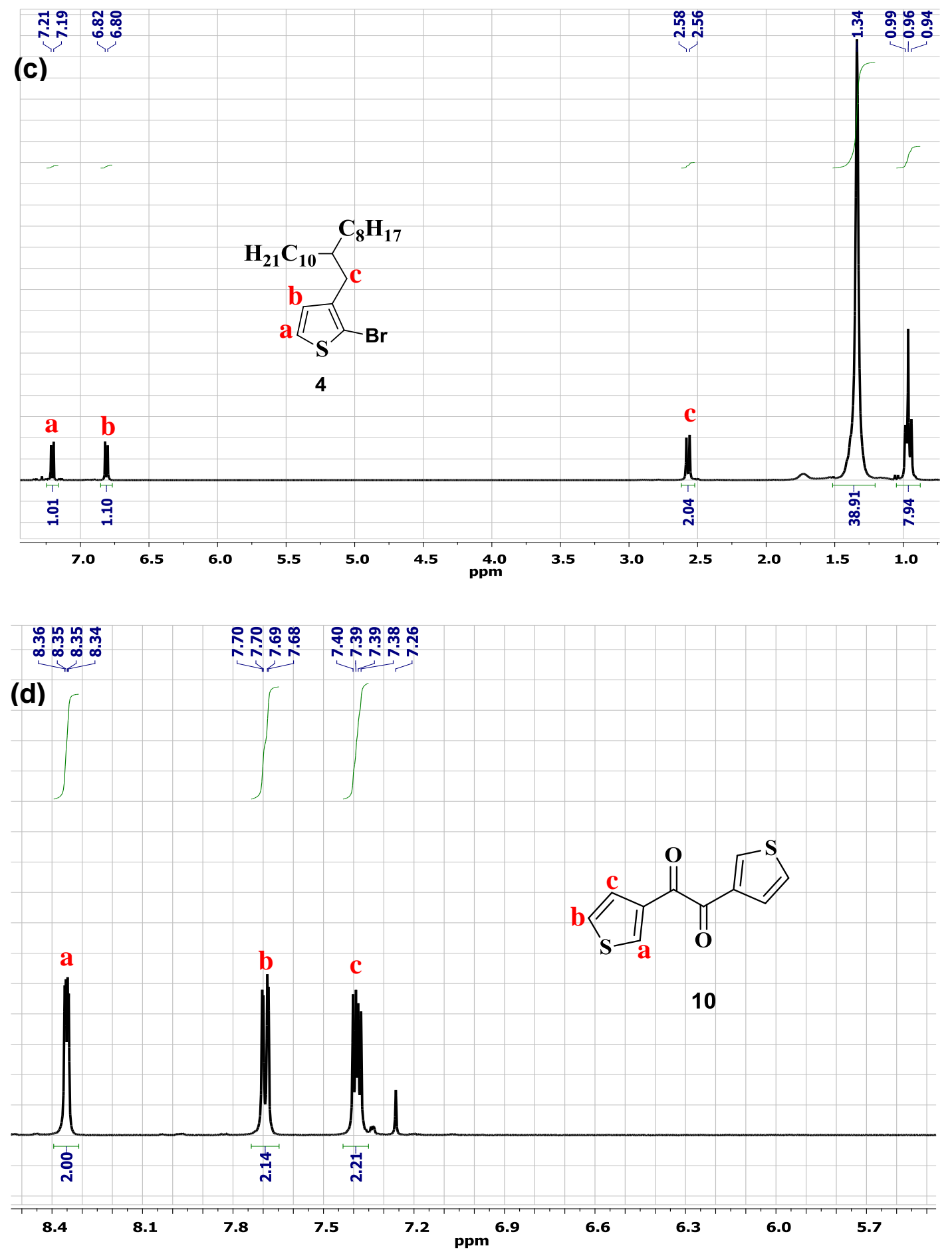

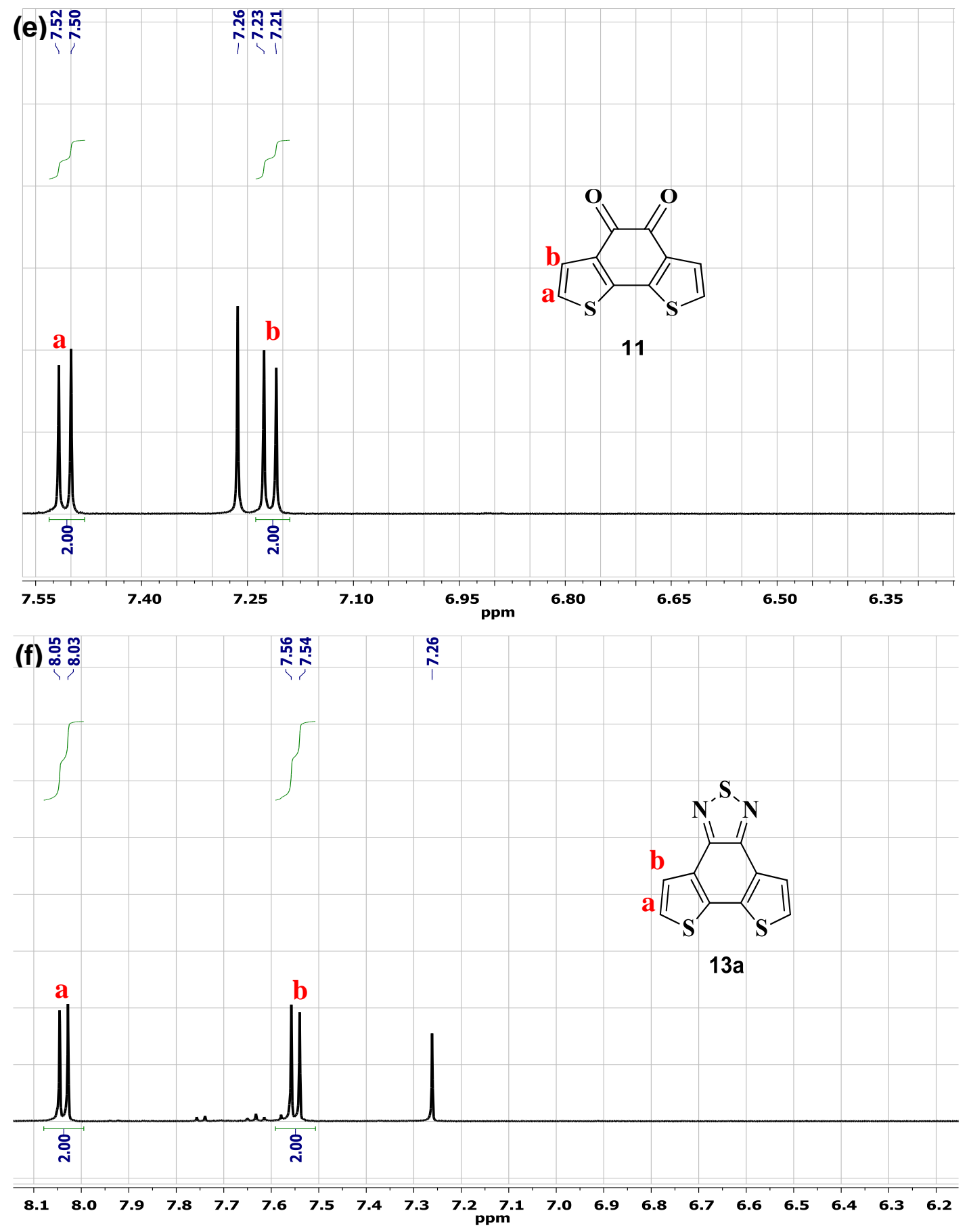




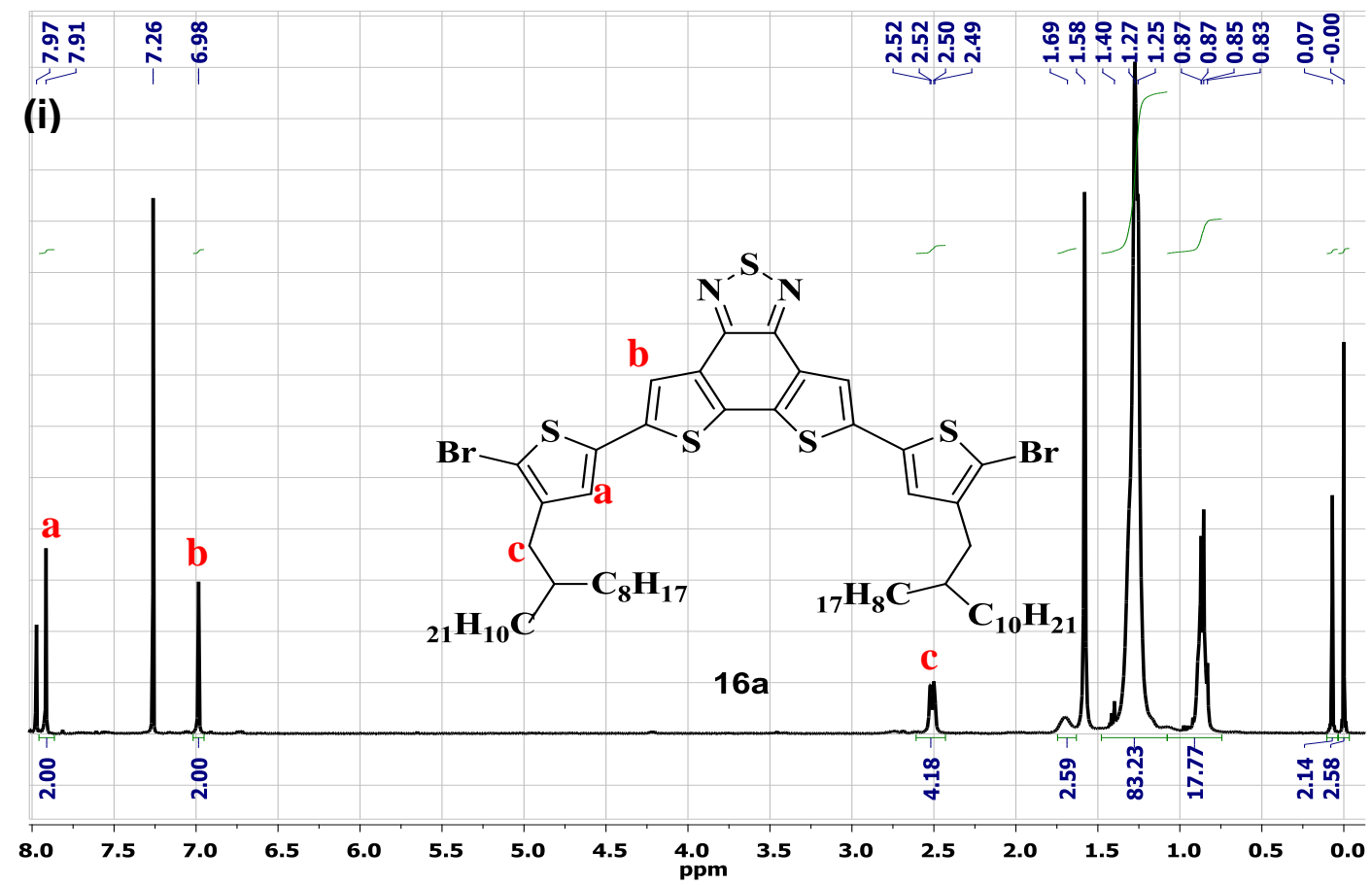

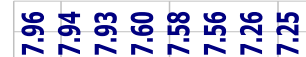

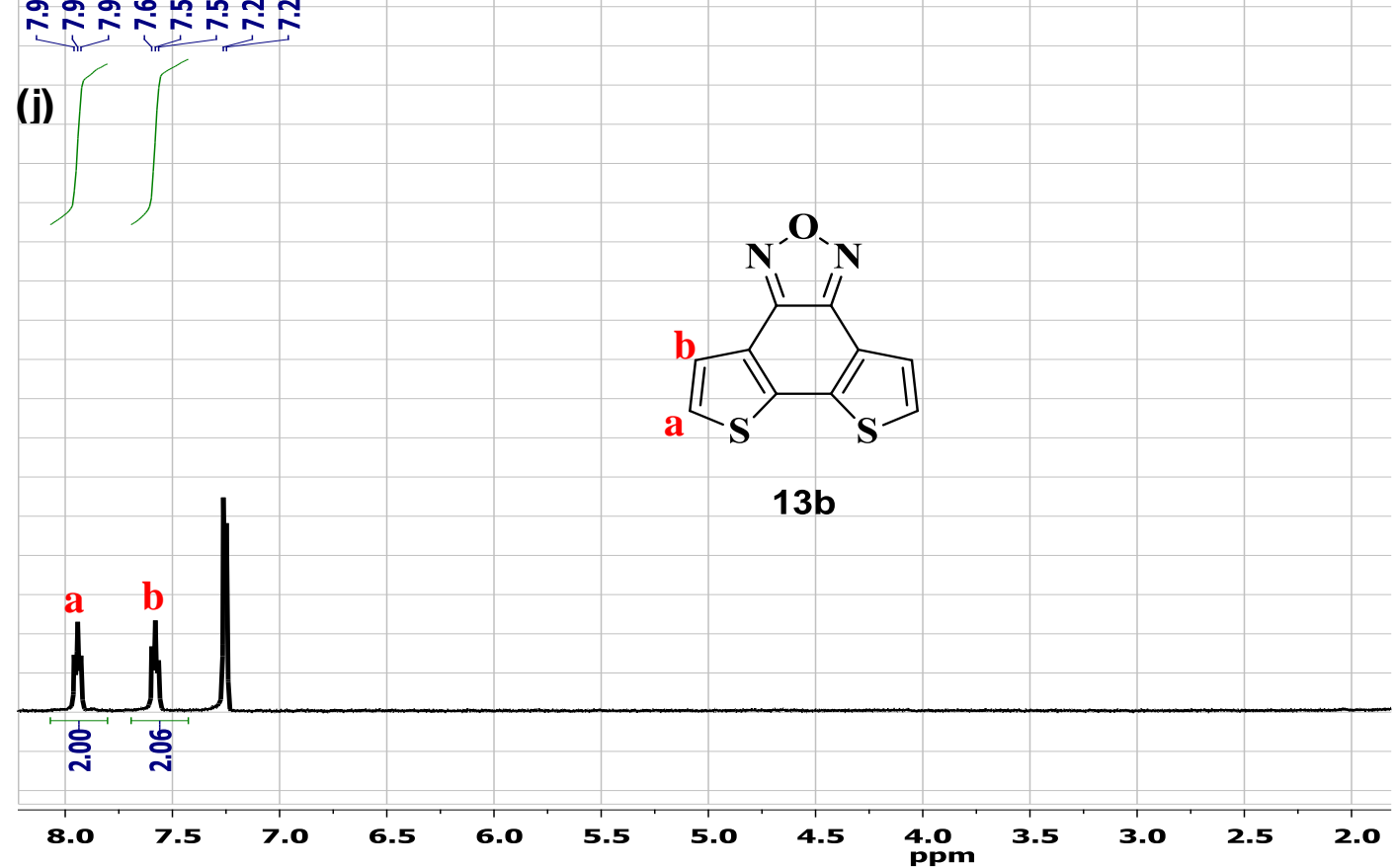



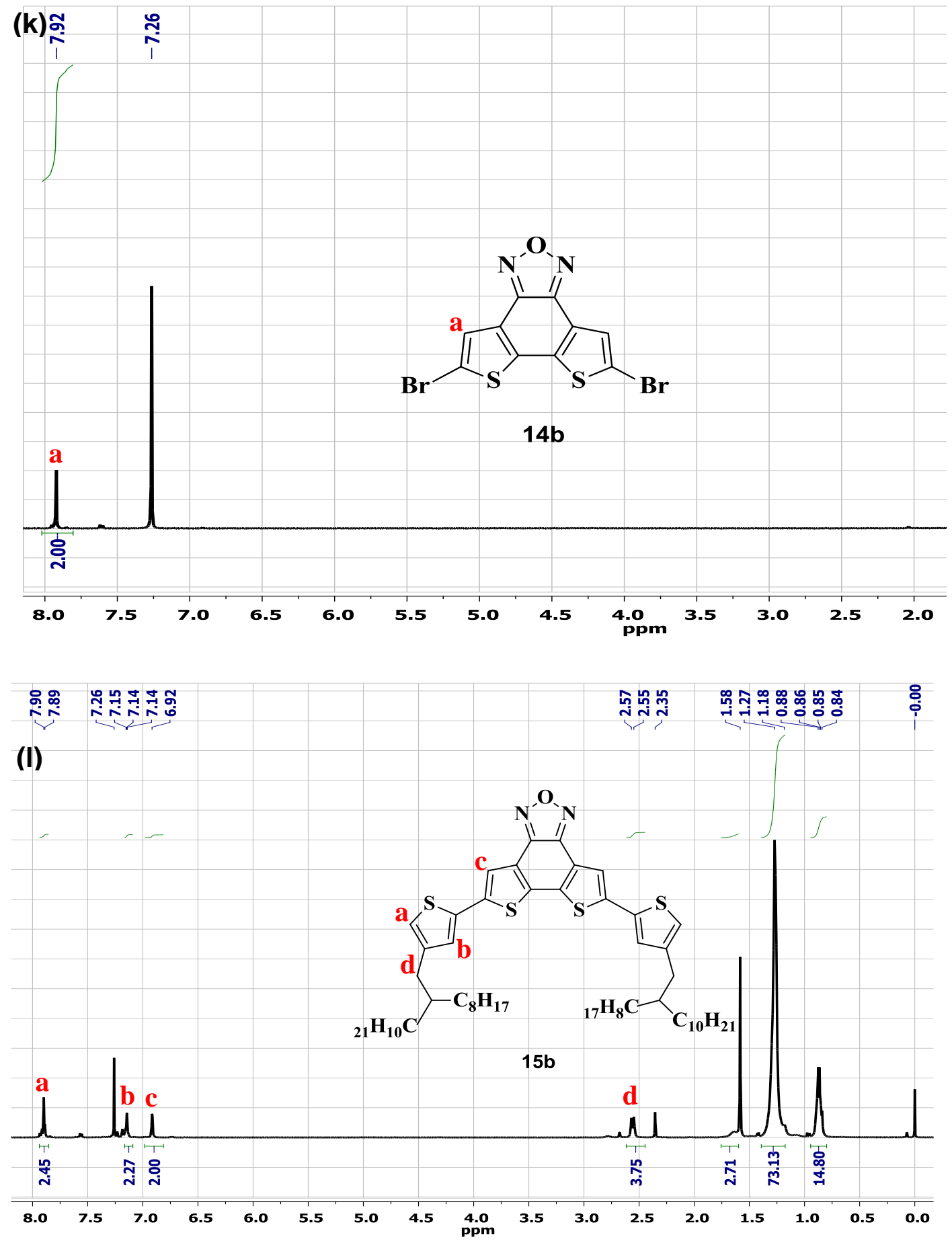


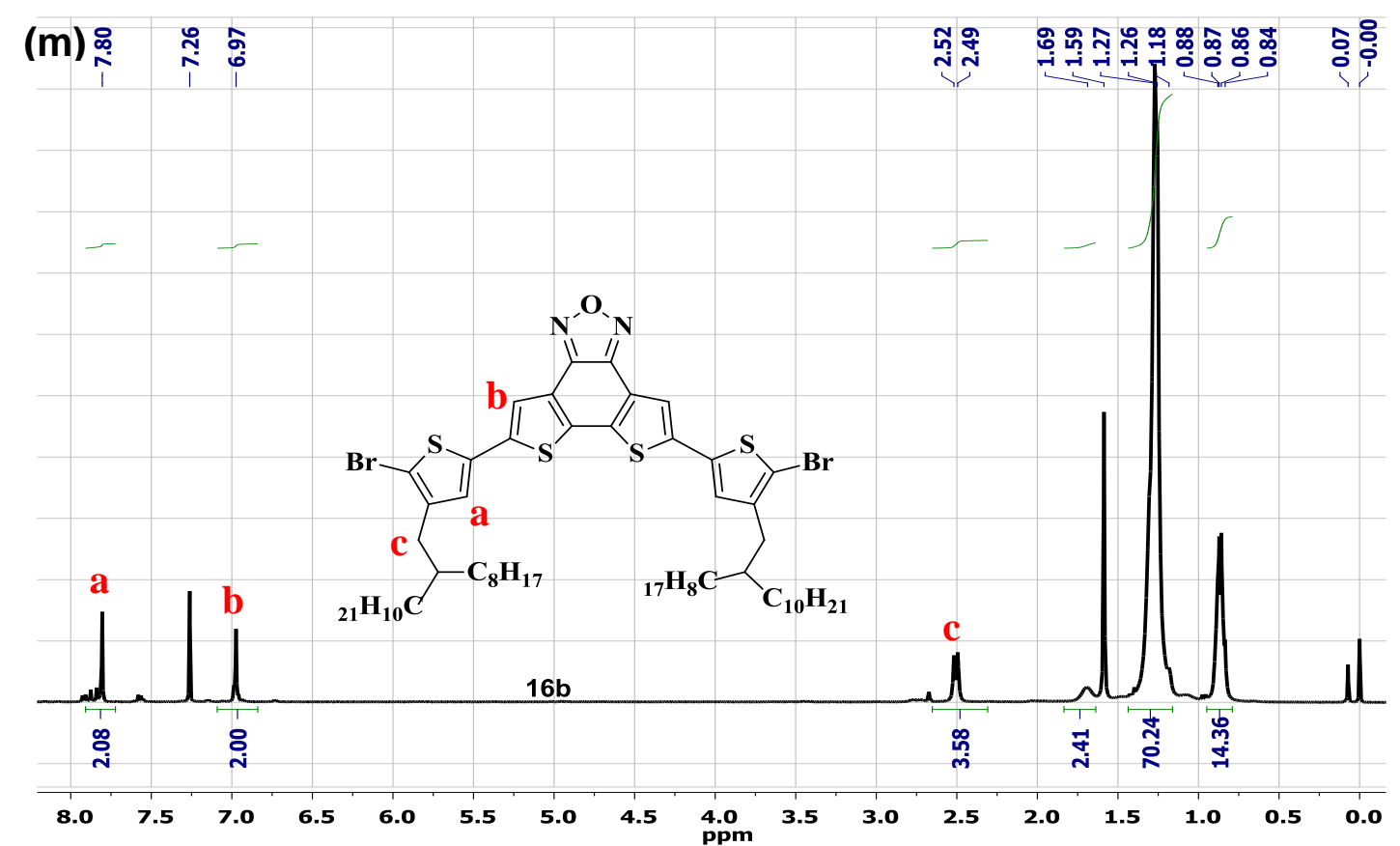

Figure 2.2. ${ }^{1} \mathrm{H}$ NMR $(300 \mathrm{MHz})$ spectra for compound $\mathbf{1}, \mathbf{3}, \mathbf{4}, \mathbf{1 0}, \mathbf{1 1}, \mathbf{1 3 a}, \mathbf{1 4 a}$,

15a, 16a, 13b, 14b, 15b, and 16b (a, b, c, d, e, f, g, h, i, j, k, l, and m), respectively, in $\mathrm{CDCl}_{3}$ solvent at R.T. 

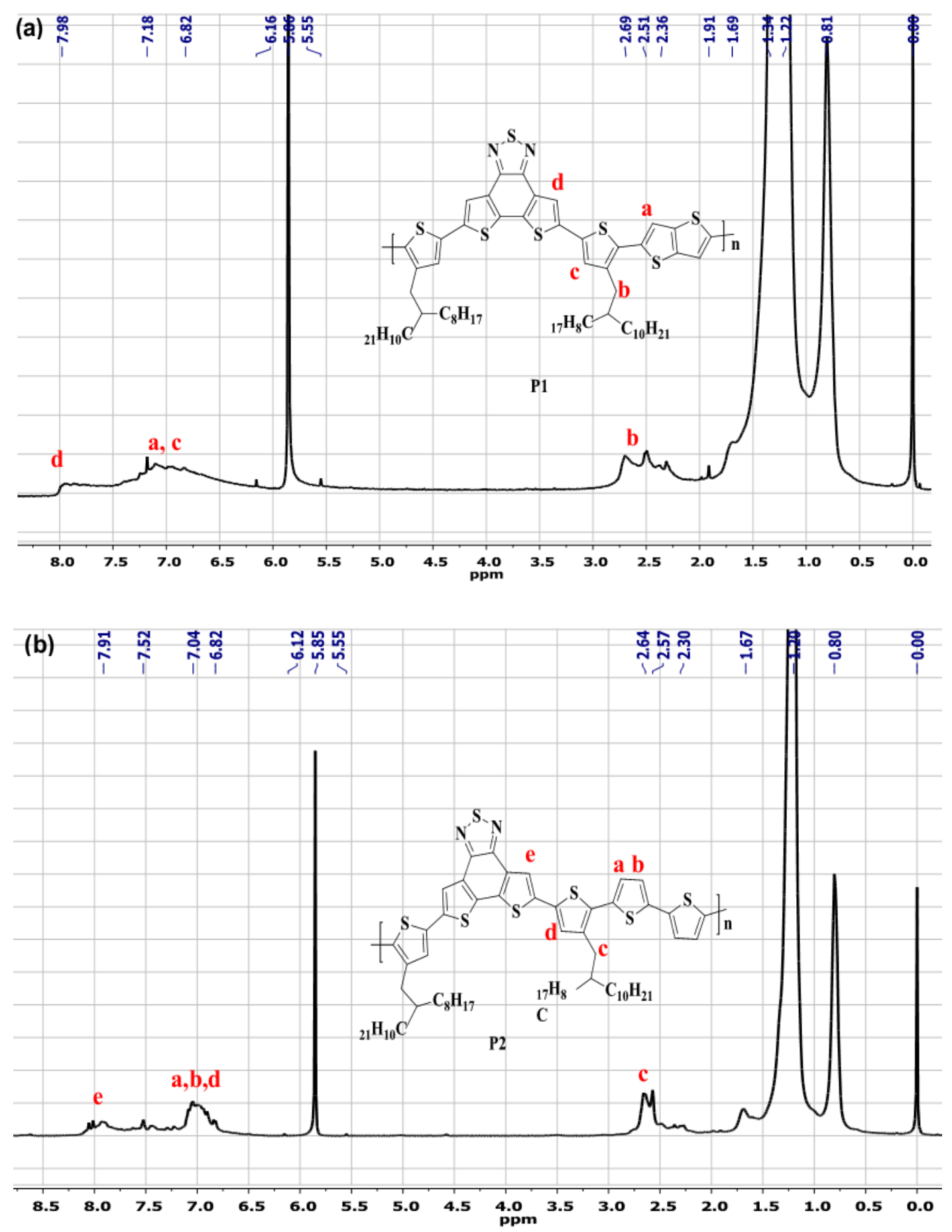

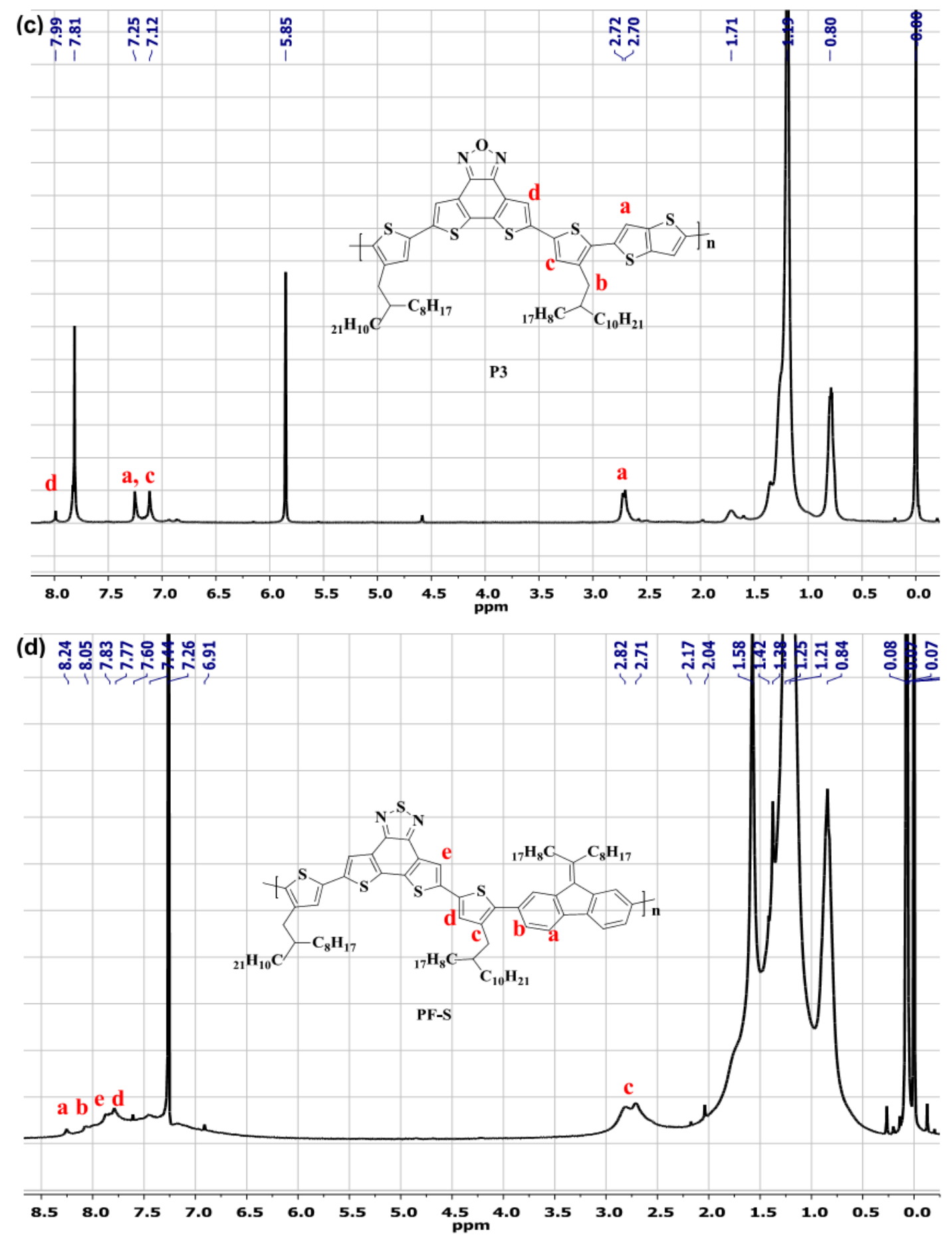


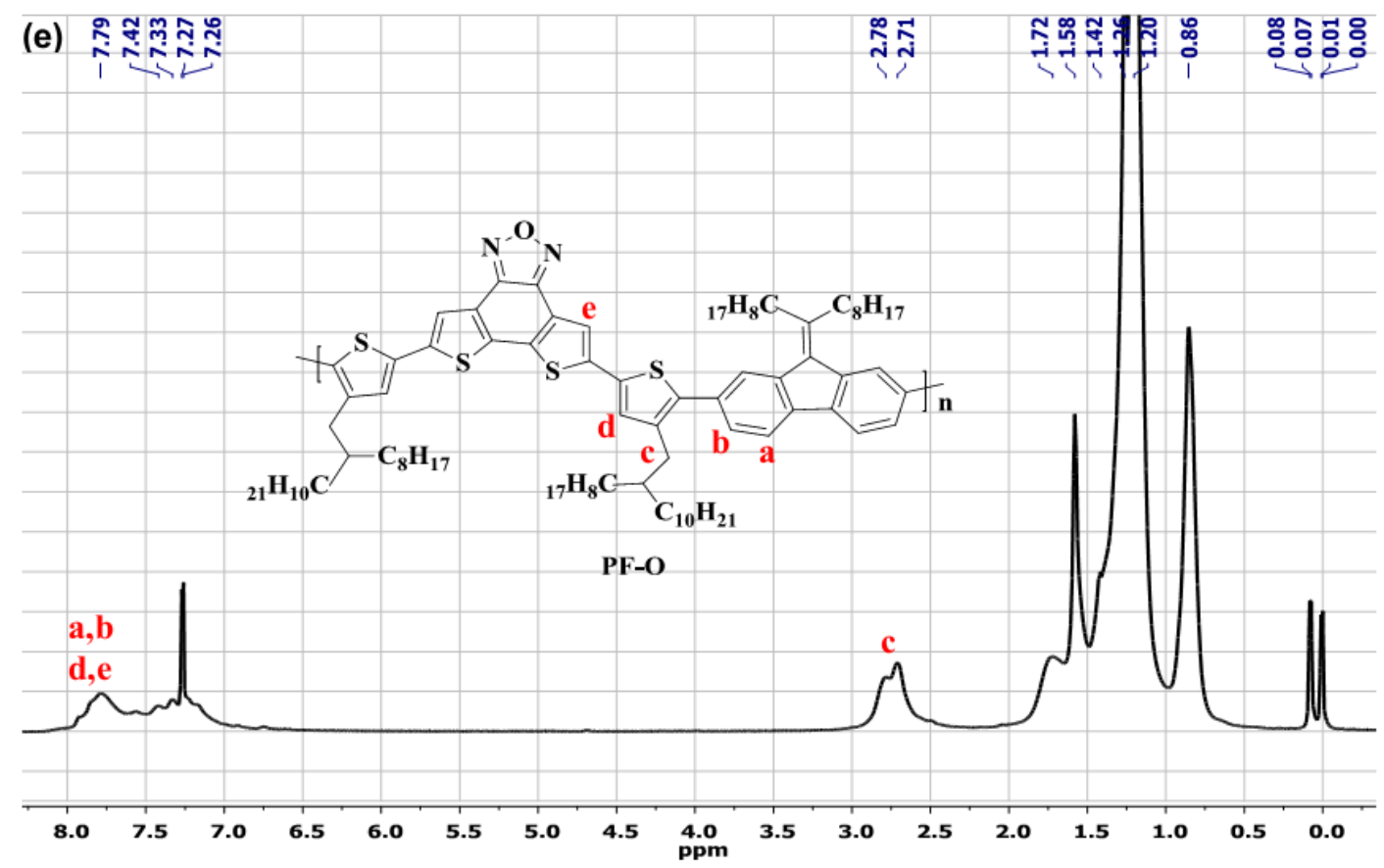

Figure 2.3. ${ }^{1} \mathrm{H}$ NMR (300 MHz) spectra for $\mathbf{P 1}(\mathrm{a}), \mathbf{P 2}$ (b), $\mathbf{P 3}$ (c) in $\mathrm{C}_{2} \mathrm{D}_{2} \mathrm{Cl}_{4}$ solvent at $100{ }^{\circ} \mathrm{C}$ and PF-S (d), PF-O (e) in $\mathrm{CDCl}_{3}$ solvent at R.T.

\subsection{Results and Discussion}

\subsubsection{Synthesis of monomers and D-A polymers}

The syntheses of monomers, 16a and 16b, are depicted in Scheme 3. The intermediate 13a was synthesized in $79 \%$ yield from the diketone $\mathbf{1 1}^{198}$ via derivatization to its corresponding diamine followed by $\mathrm{SOCl}_{2}$ treatment. The intermediate 13b was obtained from the same diketone $\mathbf{1 1}$ by refluxing it with hydroxylamine hydrochloride in a pressure vessel at $140{ }^{\circ} \mathrm{C}$ for 3 days to get a yellow 
solid. Bromination of $\mathbf{1 3 a}$ and $\mathbf{1 3 b}$ with $\mathrm{Br}_{2}$ provided the corresponding dibromides, $\mathbf{1 4 a}$ (75\% yield) and $\mathbf{1 4 b}$ (69\% yield), respectively. While 14a and $\mathbf{1 4 b}$ had limited solubility in common organic solvents except for dichlorobenzene, hot chlorobenzene, and toluene, their respective bis[4-(2-octyldodecyl)-2-thienyl)functionalized products, 15a and 15b exhibited greatly improved solubility characteristics. Subsequent bromination provided the respective dibromides, 16a, and 16b. The synthesis of 4-(2-octyldodecyl)-2-(trimethylstannyl)thiophene for the conversion of $\mathbf{1 4}$ to $\mathbf{1 5}$ is depicted in Scheme 1. Its synthesis could not be accomplished via the usual Kumada cross-coupling reaction, mostly likely due to steric interference from the long branched alkyl chain. Instead, it was obtained from the Grignard reaction (i-prMgCl$\cdot \mathrm{LiCl}$ complex) of 3-bromothiophene with 2octydodecyl aldehyde, followed by dehydroxylation with $\mathrm{AlH}_{3}$ generated in situ from a mixture of $\mathrm{LiAlH}_{4}-\mathrm{AlCl}_{3}$ and stannylation with $\mathrm{Sn}(\mathrm{Me})_{3} \mathrm{Cl}$. The chemical identities of all the intermediates $\mathbf{1}$ to $\mathbf{1 6}$ in Scheme 1 to 3 were confirmed using NMR spectroscopy.

The D-A polymers, P1, P2, and P3 were synthesized via Stille polycondensation of intermediate 16 and 2,5-bis(trimethylstannyl)-thieno[3,2-b]thiophene or 5,5'bis(trimethyl-stannyl)-2,2'-bithiophene, while both PF-S and PF-O were obtained from Suzuki polycondensation between intermediate $\mathbf{1 6}$ and dioxaborolanealkylidenefluorene in the presence of $\mathrm{Pd}\left(\mathrm{PPh}_{3}\right)_{4}$ catalyst in refluxing toluene $(110$ ${ }^{\circ} \mathrm{C}$ ) for $72 \mathrm{~h}$. All D-A polymer products were isolated via precipitation from methanol 
and purified by sequential Soxhlet extractions with methanol, acetone, hexane, and chloroform. All of the D-A polymers had displayed relatively good solubility in chlorinated solvents such as chloroform and chlorinated benzene.

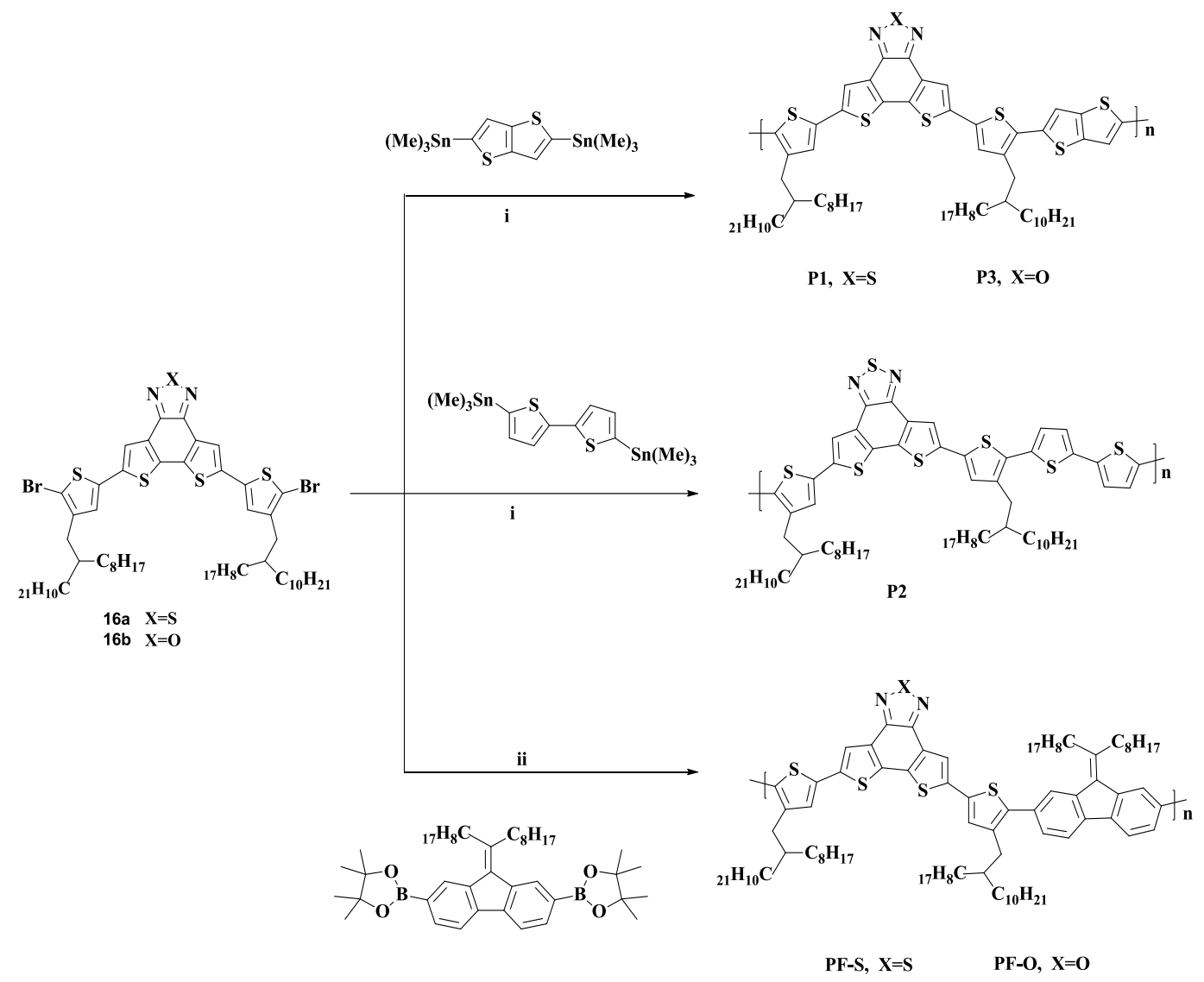

Scheme 5: Improved synthetic route to D-A polymers, P1, P2, P3, PF-S and PF-O. (i) $\mathrm{Pd}\left(\mathrm{PPh}_{3}\right)_{4}$, tolune, $110{ }^{\circ} \mathrm{C}, 68 \%$ (P1), 47\% (P2), $41 \%$ (P3). (ii) $\mathrm{Pd}\left(\mathrm{PPh}_{3}\right)_{4}$, tolune, $2 \mathrm{M} \mathrm{K}_{2} \mathrm{CO}_{3}, 110{ }^{\circ} \mathrm{C}, 70 \%$ (PF-S) and $82 \%$ (PF-O).

D-A polymers, P1 and P2, which were utilized in our OFET studies, were obtained by our previous synthesis procedure as depicted in Scheme $4 .{ }^{185}$ First, 2- 
bromo-3-(2-octyldodecyl) thiophene were coupled with the donor units 2,5bis(trimethyl stannyl)-thieno[3,2-b]thiophene or 5,5'-bis(trimethylstannyl)-2,2'bithiophene followed by stannylation and Stille polycondensation with 7a. The molecular weights and overall reaction yields of the polymers (P1 and P2 ) synthesized according to scheme 4 were lower as compared to the one obtained by using Scheme 5, this might be due to the poor solubility of the acceptor moiety compound 14a, which could affect the degree of polymerization. Therefore, scheme 4 was improved via changing the coupling sequence of alkylthiophne (compound 5) with compound $\mathbf{1 4 a}$, to get a monomer, compound $\mathbf{1 6 a}$ with greatly improved solubility and consequently higher reaction yield and molecular weight of P1 and P2 polymers were obtained.

The molecular weights and polydispersity indices (PDI) of the D-A polymers were determined by gel permeation chromatography (GPC) also known as size exclusion chromatography $(\mathrm{SCE})$ at $80{ }^{\circ} \mathrm{C}$ with a polystyrene standard calibration and $\mathrm{CHCl}_{3}$ as the eluent. Here, GPC with $\mathrm{CHCl}_{3}$ superheated well above its boiling point $\left(61^{\circ} \mathrm{C}\right)$ used as a mobile phase to avoid the overestimation of molecular weight due to aggregation of polymer in solution. ${ }^{200}$ As shown in Table 2.1, most D-A polymers have the weight-average molecular weights $\left(M_{\mathrm{w}}\right)$ as high as $20 \mathrm{kDa}$ and number-average molecular weight $\left(M_{\mathrm{n}}\right)$ ranging from 5.4 to $12.6 \mathrm{kDa}$. The polydispersity index (PDI) for all the polymers is relatively narrow, except $\mathbf{P 1}$ which shows a relatively broad PDI of 2.54 which might be due to its strong tendency to 
aggregate in solution as evidenced by its solution UV-vis absorption spectra (see Figure 2.6).

\subsubsection{Thermal Properties}

Thermal stability of all D-A polymers was investigated by thermogravimetric analysis (TGA) at a heating rate of $10{ }^{\circ} \mathrm{C} \mathrm{min}^{-1}$ under nitrogen. Figure 2.4 shows TGA where the onset decomposition temperature $\left(T_{\mathrm{d}}\right)$ with $5 \%$ weight loss is in a range of $316-416^{\circ} \mathrm{C}$, indicating that all polymers have excellent thermal stability for use in optoelectronic devices such as OPVs and OFETs.

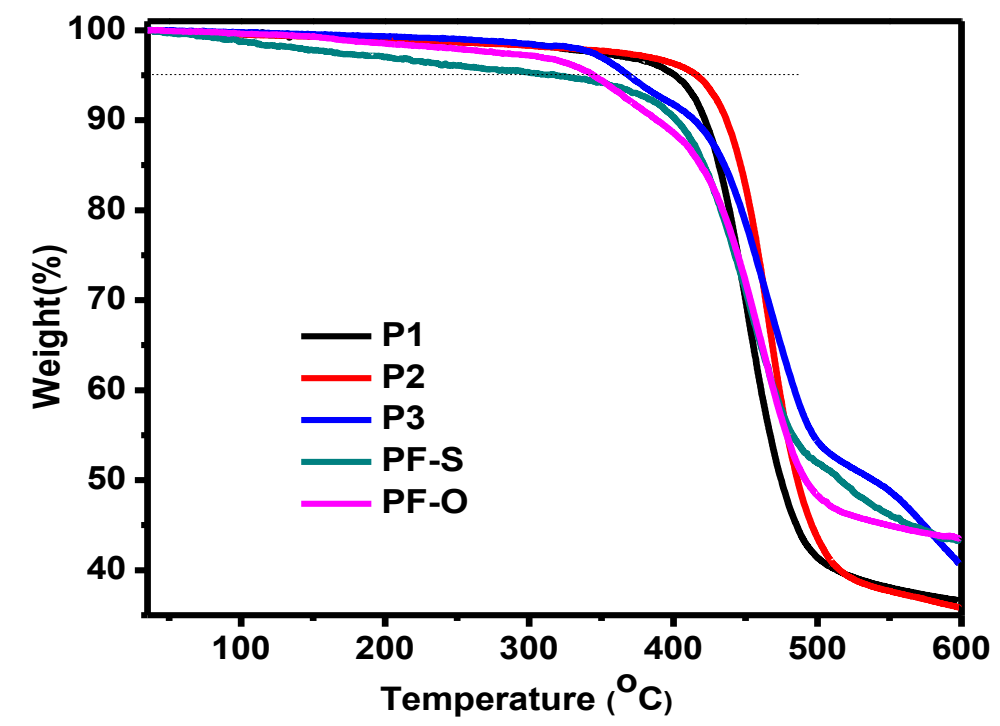

Figure 2.4. TGA plots of the five D-A polymers with a heating rate of $10{ }^{\circ} \mathrm{C} \mathrm{min}-1$ under nitrogen. 
Table 2.1. Molecular weights and thermal properties of D-A polymers.

\begin{tabular}{|l|l|l|l|l|l|}
\hline Polymer & Yield (\%) & ${ }^{\mathbf{a}} \boldsymbol{M}_{\mathbf{n}}(\mathbf{k D a})$ & ${ }^{\mathbf{a}} \boldsymbol{M}_{\mathbf{w}}(\mathbf{k D a})$ & ${ }^{\mathbf{a}} \mathbf{P D I}$ & ${ }^{\mathbf{b}} \boldsymbol{T}_{\mathbf{d}}\left({ }^{\mathbf{0}} \mathbf{C}\right)$ \\
\hline $\mathbf{P 1}$ & 68 & 8.0 & 20.5 & 2.54 & 401 \\
\hline P2 & 47 & 9.1 & 17.3 & 1.89 & 416 \\
\hline P3 & 41 & 7.6 & 14.8 & 1.93 & 368 \\
\hline PF-S & 70 & 12.6 & 17.9 & 1.42 & 316 \\
\hline PF-O & 82 & 5.4 & 8.6 & 1.59 & 343 \\
\hline
\end{tabular}

a Determined by $\mathrm{GPC}$ in $\mathrm{CHCl}_{3}$ at $80{ }^{\circ} \mathrm{C}$ using polystyrene standards. ${ }^{\mathrm{b}}$ Decomposition temperature, determined by TGA in nitrogen, based on $5 \%$ weight loss.

\subsubsection{Optical Properties}

The absorption spectra of the D-A polymers in dilute chloroform solutions and spin-coated films on quartz substrates are shown in Figure 2.5, while their respective optical data, including the maximum absorption peak wavelengths $\left(\lambda_{\max }\right)$, absorption edge wavelengths $\left(\lambda_{\text {onset }}\right)$, shoulder maximum absorption peak wavelengths $\left(\lambda_{\text {shoulder }}\right)$, and optical band gap ( $\mathrm{E}_{\mathrm{g}}{ }^{\text {opt; }}$ calculated from $\lambda_{\text {onset }}$ ), are summarized in Table 2.2. The significant red-shift and broad absorption bands of P1-3 compared to those of PF-S and PF-O could be attributed to the strong intramolecular charge transfer (ICT) interaction between the electron-rich moieties and electron-deficient segments in the former polymers. The absorption maxima for P1, P2, and $\mathbf{P 3}$ are 522, 520, $500 \mathrm{~nm}$ respectively and all of them displayed two shoulder peaks at ca. 560 and $600 \mathrm{~nm}$, 
suggesting polymer aggregation even in solution (see Figure 2.6). ${ }^{181}$ The solution absorption maxima of PF-S and PF-O were at shorter wavelength region owing to the weaker electron acceptor strength of alkylidenefluorene moiety. In thin films, as expected, all polymers (P1, P2, P3, PF-S, and PF-O) showed red-shifts for up to ca. 15 to $53 \mathrm{~nm}$, indicating higher molecular orders from intermolecular $\pi-\pi$ stacking interactions. Specifically, the oxadiazole-based polymers (P3 and PF-O) exhibited larger red-shifts than their corresponding thiadiazole-based counterparts (P1 and PFS), likely due to decreased steric interference to molecular interaction from the oxygen-containing oxadiazole-based moiety as compared to larger sulfur-containing thiadiazole-based moiety. ${ }^{201}$ Furthermore, increased intensity of 0-0 vibrational peaks $(\sim 600 \mathrm{~nm})$ relative to those of the $0-1$ vibrational peaks was observed in the film spectra of P1, P2, and P3, suggesting relatively planar polymer backbone structures and formation of J-aggregates. ${ }^{201,202}$ Finally, the absorption edges ( $\left.\lambda_{\text {onset }}\right)$ of film spectra of P1, P2, and P3 were in the range of 650 to $660 \mathrm{~nm}$, which correspond to an optical band gap ( $\left.\mathrm{E}_{\mathrm{g}}{ }^{\text {opt }}\right)$ of $1.89 \mathrm{eV}$ while $\lambda_{\text {onset }}$ for PF-S and PF-O is about $580 \mathrm{~nm}$, corresponding to $\mathrm{E}_{\mathrm{g}}{ }^{\mathrm{opt}}$ of $2.1 \mathrm{eV}$. 
Table 2.2. Optical data of the D-A polymers.

\begin{tabular}{|c|c|c|c|c|c|c|c|}
\hline \multirow[t]{2}{*}{ Polymer } & \multicolumn{3}{|c|}{ Solution } & \multicolumn{3}{|l|}{ Film } & \multirow[b]{2}{*}{$\begin{array}{l}{ }^{d} \mathbf{E}_{\mathrm{g}} \text { opt } \\
(\mathrm{eV})\end{array}$} \\
\hline & $\begin{array}{l}{ }^{\mathbf{a}} \lambda_{\max } \\
{[\mathrm{nm}]}\end{array}$ & $\begin{array}{l}{ }^{b} \lambda_{\text {shold }} \\
{[\mathbf{n m}]}\end{array}$ & $\begin{array}{l}\mathbf{c}_{\text {onset }} \\
{[\mathbf{n m}]}\end{array}$ & $\begin{array}{l}{ }^{\mathbf{a}} \lambda_{\max } \\
{[\mathrm{nm}]}\end{array}$ & $\begin{array}{l}{ }^{\mathbf{b}} \lambda_{\text {shold }} \\
{[\mathbf{n m}]}\end{array}$ & $\begin{array}{l}\mathbf{c}_{\text {onset }} \\
{[\mathrm{nm}]}\end{array}$ & \\
\hline P1 & 522 & 597 & 660 & 552 & 597 & 658 & 1.88 \\
\hline $\mathbf{P 2}$ & 520 & 600 & 651 & 552 & 592 & 654 & 1.89 \\
\hline P3 & 500 & 601 & 655 & 553 & 595 & 655 & 1.89 \\
\hline PF-S & 480 & - & 570 & 495 & - & 578 & 2.15 \\
\hline PF-O & 465 & - & 547 & 485 & - & 581 & 2.13 \\
\hline
\end{tabular}

${ }^{\mathrm{a} A b s o r p t i o n}$ maxima. ${ }^{\mathrm{b}}$ Absorption maxima at shoulder peak. ${ }^{\mathrm{c}}$ The onset edge absorption. ${ }^{\mathrm{d}}$ Estimated from the onset edge of absorption in films $\left(\lambda_{\text {onset }}\right):{ }^{d} E_{g}{ }^{o p t}(e V)=$ $1240 / \lambda_{\text {onset }}[\mathrm{nm}]$


Figure 2.5. UV-vis absorption spectra of D-A polymers (a) in chloroform solution and (b) thin films for D-A polymers, P1, P2, P3, PF-S, and PF-O. 

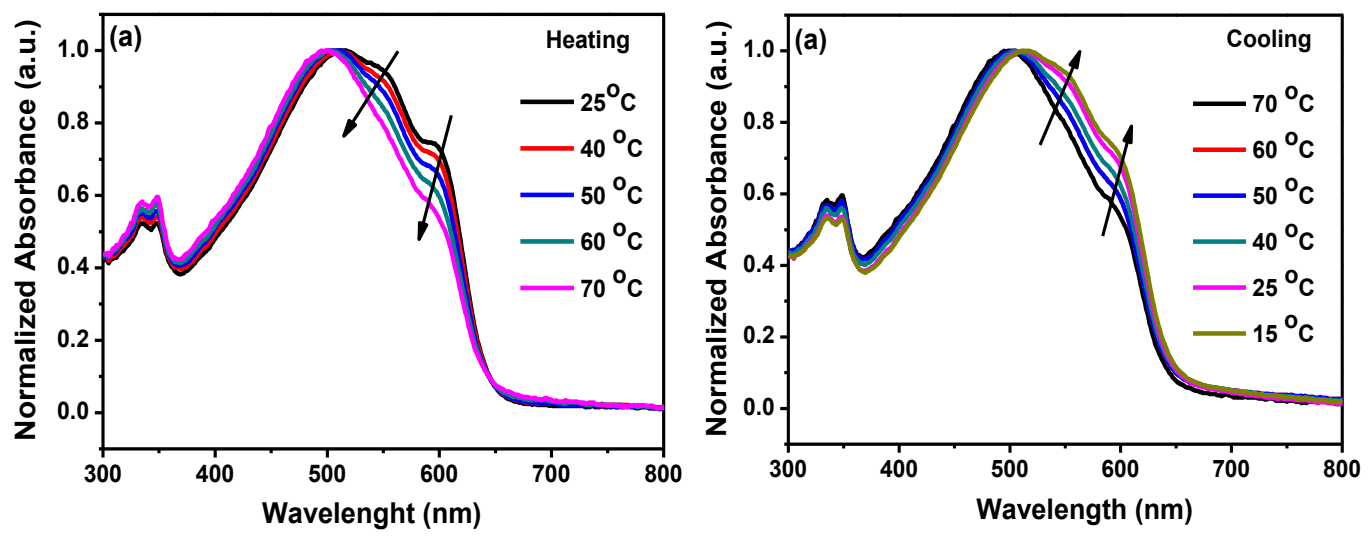

Figure 2.6. The evolutions of UV-vis absorption spectra of $\mathbf{P 1}$ in $\mathrm{CHCl}_{3}$ during (a) heating process from 25 to $70{ }^{\circ} \mathrm{C}$ and (b) cooling process from 70 to $15^{\circ} \mathrm{C}$.

\subsubsection{Electrochemical Properties}

The cyclic voltammograms $(\mathrm{CV})$ were carried out on D-A polymer films to examine their electronic energy levels (see Figure 2.7). The highest occupied molecular orbital (HOMO) and lowest unoccupied molecular orbital (LUMO) energy levels were calculated from the onset oxidation potentials $\left(\mathrm{E}_{\mathrm{ox}}{ }^{\text {onset }}\right)$ and the onset reduction potentials $\left(\mathrm{E}_{\text {red }}{ }^{\text {onset }}\right),{ }^{43}$ respectively and summarized in Table 2.3. Within experimental errors, the band gaps calculated from the measured $\mathrm{CV}\left(\mathrm{E}_{\mathrm{g}}{ }^{\mathrm{elc}}\right.$ ) data were essentially similar to those estimated from optical absorption edges (Table 2.2). It is obvious that the replacement of sulfur atom of thiadiazole moiety with an oxygen atom to form oxadiazole moiety had a noticeable effect on the energy levels of the resulting D-A polymers. Thus, with the same donor moiety, the oxadiazolebased polymer was observed to display a slightly lower LUMO energy level than its 
corresponding thiadiazole-based polymer, attributable to the stronger electronegativity of oxygen.

Table 2.3. Electrochemical properties of D-A polymers.

\begin{tabular}{|l|l|l|l|l|l|l|}
\hline Polymer & $\begin{array}{l}\mathbf{E}_{\mathbf{o x}}{ }^{\text {onset }} \\
(\mathbf{V})\end{array}$ & $\begin{array}{l}\mathbf{E}_{\mathbf{r e d}^{\text {onset }}} \\
(\mathbf{V})\end{array}$ & $\begin{array}{l}\mathbf{a}^{\mathbf{H O M O}} \\
(\mathbf{e V})\end{array}$ & $\begin{array}{l}\mathbf{b}^{\mathbf{b}} \mathbf{L M O} \\
(\mathbf{e V})\end{array}$ & $\begin{array}{l}\mathbf{L U M O}^{\text {elc }} \\
(\mathbf{e V})\end{array}$ & $\begin{array}{l}\mathbf{E}_{\mathbf{g}} \text { elc } \\
(\mathbf{e V})\end{array}$ \\
\hline $\mathbf{P 1}$ & 1.02 & -0.77 & -5.42 & -3.54 & -3.63 & 1.79 \\
\hline $\mathbf{P 2}$ & 0.93 & -0.75 & -5.33 & -3.44 & -3.65 & 1.68 \\
\hline P3 & 1.00 & -0.74 & -5.40 & -3.51 & -3.66 & 1.74 \\
\hline PF-S & 1.42 & -0.68 & -5.82 & -3.67 & -3.72 & 2.10 \\
\hline PF-O & 1.34 & -0.66 & -5.74 & -3.61 & -3.74 & 2.00 \\
\hline
\end{tabular}

${ }^{\mathrm{a}} \mathrm{HOMO}^{\mathrm{ele}}=-\mathrm{e}(\mathrm{Eox}+4.4)(\mathrm{eV}) \cdot{ }^{\mathrm{b}} \mathrm{LUMO}^{\mathrm{opt}}=\mathrm{Eg}^{\mathrm{opt}}+\mathrm{E}_{\text {Hомо. }}$

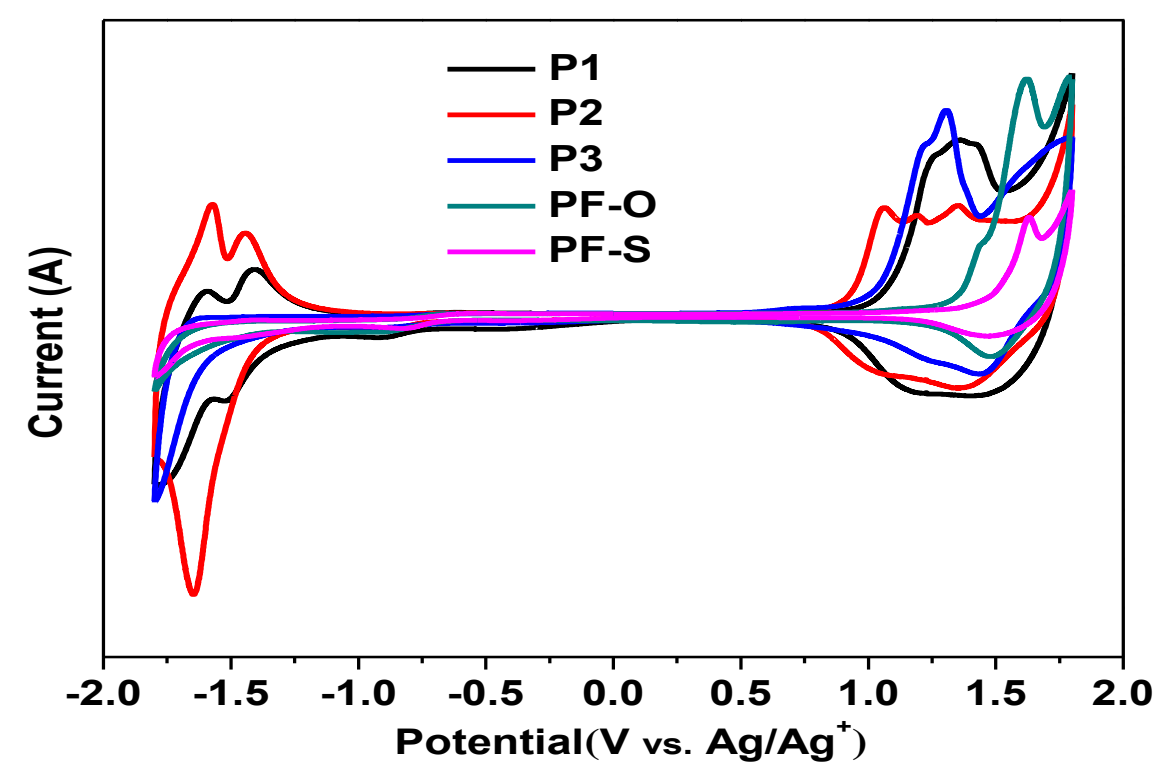

Figure 2.7. The cyclic voltammograms of P1, P2, P3, PF-S, and PF-O films on a platinum electrode measured in $0.1 \mathrm{~mol} / \mathrm{lit} \mathrm{Bu}_{4} \mathrm{NPF}_{6}$ acetonitrile solutions at a scan rate of $50 \mathrm{mVs}^{-1}$. 


\subsubsection{Photovoltaic Properties of P1, P2, P3, PF-S, and PF-O Polymers}

The photovoltaic properties of the D-A polymers were investigated in inverted bulk heterojunction organic photovoltaic (inverted BHJ-OPV) devices using a solar simulator (San_El Electric, XEC-301S) and Agilent U2722A Modular Source Measure Unit. The device structure had the configuration of ITO/ZnO/active layer/ $\mathrm{MoO}_{3} / \mathrm{Ag}$ with the active layer being composed of D-A polymer as the electron donor and PCBM as an electron acceptor. The observed photovoltaic properties, i.e., open circuit voltage $\left(\mathrm{V}_{\mathrm{oc}}\right)$, short-circuit current $\left(\mathrm{J}_{\mathrm{sc}}\right)$, fill factor $(\mathrm{FF})$, and power conversion efficiency (PCE) are summarized in Table $2.4 \& 3.5$. The PCEs ranging from $0.21 \%$ to $2.2 \%$ were recorded for these devices. It was noted that the devices with active layer spin cast at a spin speed of $1000 \mathrm{rpm}$ (see Figure 2.8 and Table 2.4) displayed higher PCEs than those at spinning speed of $500 \mathrm{rpm}$ (see Figure 2.9 and Table 2.5), which could be attributed to the better film quality of the higher spinning speed. As can be seen from the current density-voltage plots of Figure 2.8, the devices using PF-S and PF-O as donor polymers exhibited much higher $\mathrm{V}_{\text {oc }}(0.93$ and $0.98 \mathrm{~V}$, respectively) than those with P1, P2, and P3 (6.8 V) as donor polymers. These are consistent with their HOMO levels since $\mathrm{V}_{\mathrm{oc}}$ is proportional to the energy difference between the HOMO level of donor and LUMO level of the acceptor (PCBM). ${ }^{43}$ However, the poor PCE value for PF-S and PF-O could be due to low $\mathbf{J}_{\mathrm{sc}}$ (see Table 2.4), as the AFM images show that, they form large aggregates than much- 
needed nanoscale bicontinuous morphology for better charge transport and separation at the polymer: PCBM interface.

The device with donor polymer, P2, afforded the best PCE of $2.2 \%$ and $\mathrm{J}_{\mathrm{sc}}(4.7$ $\mathrm{mA} / \mathrm{cm}^{2}$ ) comparable to that of $\mathbf{P 1}$, which might be due to its higher fill factor $(66 \%)$. It has been suggested that series resistance $\left(R_{s}\right)$ and shunt resistance $\left(R_{s h}\right)$ are two of the factors that determine $F F,{ }^{203}$ thus, a device having lower $\mathrm{R}_{\mathrm{s}}$ and higher $\mathrm{R}_{\mathrm{sh}}$ would yield higher FF and improved PCE. P2 showed smallest $R_{s}$ of $8.2 \Omega \cdot \mathrm{cm}^{2}$ and a relatively higher $\mathrm{R}_{\mathrm{sh}}$ of $1.9 \mathrm{k} \Omega \cdot \mathrm{cm}^{2}$ than the other four polymers, while PF-O, which gave the lowest PCE of $0.21 \%$, had the highest $R_{\mathrm{s}}$ of 1.9 and $\mathrm{R}_{\mathrm{sh}}$ of $2.3 \mathrm{k} \Omega \cdot \mathrm{cm}^{2}$. Therefore, the observed PCE values for all five polymers were quite consistent with their FF, $\mathbf{R}_{s}$ and $\mathbf{R}_{\text {sh }}$ values. The highest PCE for $\mathbf{P} 2$ may be attributed to its lower band-gap and broader absorptions which enable more efficient solar energy harvesting.

The incident photon to current efficiency, IPCE, of the devices for P1, P2, and P3 (see Figure 2.10) shows reasonably wide external quantum efficiency (EQE) graphs in the visible region (300-700 nm) with large EQE values in the 300-730 nm region and lower values beyond $730 \mathrm{~nm}$. The $\mathbf{P 1}$ devices exhibited the highest EQE (up to $47.5 \%$ ) at $380 \mathrm{~nm}$, in good agreement with the result for the highest photocurrent value, $\mathrm{J}_{\mathrm{sc}}$ of $4.71 \mathrm{~mA} / \mathrm{cm}^{2}$ whereas, $\mathbf{P 2}$ and $\mathbf{P 3}$ showed EQE values of $45.5 \%$ and $37.4 \%$, respectively. 
Table 2.4. Photovoltaic performance of $\mathrm{D}-\mathrm{A}$ polymers with $\mathrm{PC}_{71} \mathrm{BM}$ under illumination of AM 1.5G, $940 \mathrm{~W} / \mathrm{m}^{2}$ (Active layer (spin seeped at $1000 \mathrm{rpm}$ ): D-A Polymer/PC ${ }_{71} \mathrm{BM}$ (1/2 by weight) with $3 \%$ of $\mathrm{DIO} ; 10-12$ devices were evaluated for each polymer).

\begin{tabular}{|l|l|l|l|l|l|l|}
\hline Polymer & $\begin{array}{l}\mathbf{V}_{\text {oc }} \\
(\mathbf{V})\end{array}$ & $\begin{array}{l}\mathbf{J}_{\text {sc }} \\
\left(\mathbf{m A} \mathbf{m} / \mathbf{c m}^{\mathbf{2}}\right)\end{array}$ & $\begin{array}{l}\mathbf{F F} \\
(\mathbf{\%})\end{array}$ & $\begin{array}{l}\mathbf{P C E} \\
(\mathbf{\%})\end{array}$ & $\begin{array}{l}\mathbf{R}_{\mathbf{s}} \\
\left(\mathbf{k} \mathbf{\Omega} \cdot \mathbf{c m}^{\mathbf{2}}\right)\end{array}$ & $\begin{array}{l}\mathbf{R}_{\text {sh }} \\
\left(\mathbf{k} \mathbf{\Omega} \cdot \mathbf{c m}^{\mathbf{2}}\right)\end{array}$ \\
\hline P1 & 0.68 & 4.7 & 56 & 1.9 & 0.010 & 1.1 \\
\hline P2 & 0.68 & 4.7 & 66 & 2.2 & $8.2 \times 10^{-3}$ & 1.9 \\
\hline P3 & 0.68 & 3.9 & 55 & 1.6 & 0.019 & 0.98 \\
\hline PF-S & 0.93 & 1.7 & 52 & 0.88 & 0.80 & 2.1 \\
\hline PF-O & 0.98 & 0.68 & 30 & 0.21 & 1.9 & 2.3 \\
\hline
\end{tabular}


Figure 2.8. Current density-voltage curves of photovoltaic devices with an active layer of D-A polymer/ $\mathrm{PC}_{71} \mathrm{BM}(1 / 2$ by weight $)$ and $3 \%$ of DIO additive at $1000 \mathrm{rpm}$ spin speed. 
Table 2.5. Photovoltaic performance of $\mathrm{D}-\mathrm{A}$ polymers with $\mathrm{PC}_{71} \mathrm{BM}$ (1:2 by weight) and $3 \%$ of DIO additive as active layers (spin seeped at $500 \mathrm{rpm}$ ) under the illumination of AM 1.5G, $940 \mathrm{~W} / \mathrm{m}^{2}$. $(10-12$ devices were evaluated for each polymer).

\begin{tabular}{|l|l|l|l|l|l|l|l|}
\hline Polymer & $\begin{array}{l}\mathbf{V}_{\text {oc }} \\
(\mathbf{V})\end{array}$ & $\begin{array}{l}\mathbf{J}_{\text {sc }} \\
\left(\mathbf{m A} / \mathbf{c m}^{2}\right)\end{array}$ & $\begin{array}{l}\mathbf{F F} \\
\mathbf{( \% )}\end{array}$ & $\begin{array}{l}\mathbf{P C E} \\
\mathbf{( \% )}\end{array}$ & $\begin{array}{l}\mathbf{R}_{\mathbf{s}} \\
\left(\mathbf{k} \mathbf{\Omega} \cdot \mathbf{c m}^{2}\right)\end{array}$ & $\begin{array}{l}\mathbf{R}_{\text {sh }} \\
\left(\mathbf{k} \mathbf{\Omega} \cdot \mathbf{c m}^{2}\right)\end{array}$ & $\begin{array}{l}\mathbf{R}_{\mathbf{m s}} \\
(\mathbf{n m})\end{array}$ \\
\hline $\mathbf{P 1}$ & 0.68 & 4.4 & 47 & 1.48 & 0.018 & 0.50 & 1.86 \\
\hline $\mathbf{P 2}$ & 0.66 & 4.4 & 60 & 1.85 & $9.3 \times 10^{-3}$ & 1.2 & 4.57 \\
\hline P3 & 0.61 & 3.0 & 51 & 0.98 & 0.030 & 0.71 & 3.50 \\
\hline PF-S & 0.93 & 1.4 & 52 & 0.74 & 0.84 & 2.4 & 3.93 \\
\hline PF-O & 0.82 & 0.50 & 34 & 0.15 & 1.1 & 2.9 & 2.52 \\
\hline
\end{tabular}


Figure 2.9. Current density-voltage curves of photovoltaic devices with active layers of P1, P2, and $\mathbf{P 3}$ and $\mathrm{PC}_{71} \mathrm{BM}$ acceptor (1:2 by weight) with $3 \%$ of DIO additive at $500 \mathrm{rpm}$ spin speed. 


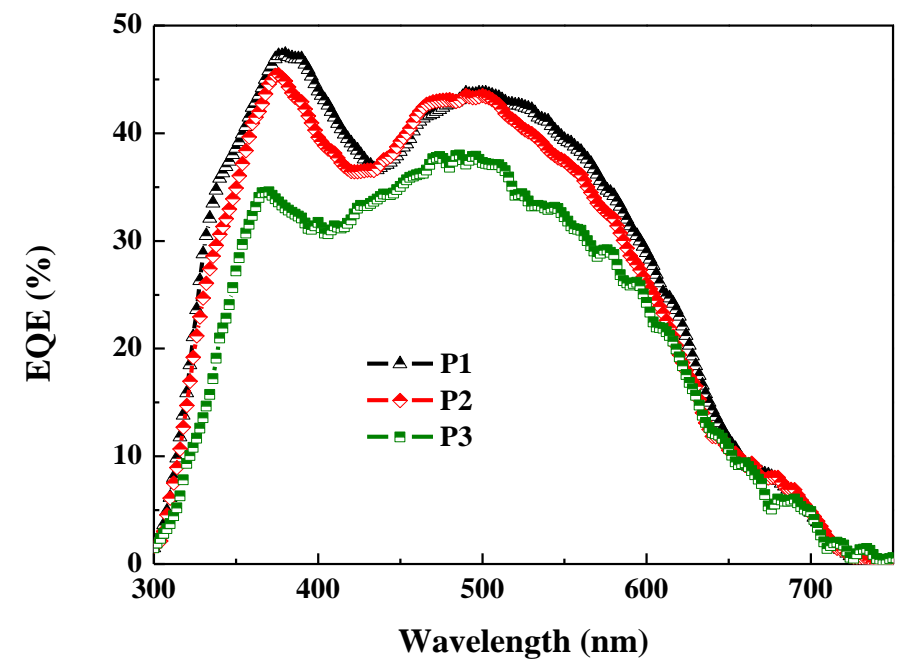

Figure 2.10. IPCE spectra of the OPV devices using P1, P2, and P3 as donor polymers and $\mathrm{PC}_{71} \mathrm{BM}$ as acceptor.

\subsubsection{Field Effect Transistor Properties of P1, P2, P3, PF-S, and PF-O Polymers}

The field-effect transistor properties of D-A polymers were evaluated using a bottom-gate, top-contact transistor configuration. A heavily n-doped silicon wafer $<100>$ with a $300-\mathrm{nm}$ thermal silicon dioxide $\left(\mathrm{SiO}_{2}\right)$ was used as the substrate/gate electrode. The surface of $\mathrm{SiO}_{2} / \mathrm{Si}$ substrate was modified with either octyltrichlorosilane (OTS-8) or octadecyltrichlorosilane (OTS-18) to enhance transistor performance. The D-A polymer was dissolved in chlorobenzene (3 $\mathrm{mg} / \mathrm{mL}$ ) and filtered through a syringe fitted with a 10-micron filter. The filtered solution was spin coated on top of OTS-8- or OTS-18-modified $\mathrm{SiO}_{2}$ surface, vacuum dried at $60{ }^{\circ} \mathrm{C}$ for several hours. The devices were optionally annealed at 
$120{ }^{\circ} \mathrm{C}$ for 30 min when required. A series of gold source/drain electrode pairs were deposited by vacuum thermal evaporation through a shadow mask, thus creating a set of OFET devices with a various channel length (L) and width (W) dimensions for electrical evaluation. All the polymers had shown p-type semiconductor behavior, and their OFET characteristics are summarized in Table 2.6 for devices treated with OTS-8 and OTS-18, respectively. Typical Transfer and output characteristics, as well as hysteresis behavior of OFET devices with $\mathbf{P 2}$ as channel semiconductor on the OTS-18-modified substrate, are provided in Figure 2.12 (d, e \& f). The transfer and output characteristics and hysteresis behaviors of all other D-A polymers are given in Figure $2.11 \& 2.12$. The extracted field-effect mobility, current on/off ratio and threshold voltage of OFET devices on OTS-8- and OTS-18-modifed substrates is summarized in Table 2.6 and 3.7, respectively. As can be noted, the results showed that $\mathbf{P 1}, \mathbf{P 2}$, and $\mathbf{P 3}$ gave essentially the same field-effect properties, with $\mathbf{P 2}$ performed slightly better and P3 slightly poorer. On the other hand, both PF-S and PF-O gave significantly poorer OFET performance, which may be attributed to the donor moiety, alkylidenefluorene, being not a strong donor. In addition, we observed that those devices built on ODTS-18-modified $\mathrm{SiO}_{2}$ substrates afforded much better performance than those on OTS-8-modified substrates. Both mobility and on/ratio are much higher in devices on OTS-18-modified substrates, which are in agreement with the higher efficiency of the octadodecyl chain in facilitating molecular ordering of the D-A polymers. In addition, noticeable improvements in mobility and on/off 
ratio were noted after the devices were thermally annealed at $120{ }^{\circ} \mathrm{C}$ as thermal treatment was expected to further promote molecular ordering of D-A polymers. The highest mobility of $2.6 \times 10^{-2} \mathrm{~cm}^{2} \mathrm{~V}^{-1} \mathrm{~s}^{-1}$ and on/ratio of $3 \times 10^{5}$ were obtained from devices using $\mathbf{P 2}$ as channel semiconductor.

Table 2.6. Performance characteristics of OFET devices with D-A polymer semiconductor on an OTS-8-treatd substrate fabricated and measured under ambient conditions.

\begin{tabular}{|c|c|c|c|c|}
\hline \multirow[t]{2}{*}{ Polymer } & \multicolumn{2}{|c|}{ Average Mobility* $\left(\mathrm{cm}^{2} \mathbf{V}^{-1} \mathbf{s}^{-1}\right)$} & \multirow{2}{*}{\multicolumn{2}{|c|}{$\begin{array}{l}V_{\text {th }}(\mathrm{V}) \text { On/off ratio } \\
\text { Annealed at } 120^{\circ} \mathrm{C}\end{array}$}} \\
\hline & As fabricated, $60^{\circ} \mathrm{C}$ & Annealed at $120^{\circ} \mathrm{C}$ & & \\
\hline $\mathbf{P 1}$ & $0.7 \times 10^{-2}\left(0.8 \times 10^{-2}\right)$ & $1.1 \times 10^{-2}\left(1.3 \times 10^{-2}\right)$ & -6.0 & $2 \times 10^{4}$ \\
\hline P2 & $1.1 \times 10^{-2}\left(1.2 \times 10^{-2}\right)$ & $1.6 \times 10^{-2}\left(1.7 \times 10^{-2}\right)$ & -4.0 & $6 \times 10^{4}$ \\
\hline P3 & $0.5 \times 10^{-2}\left(0.6 \times 10^{-2}\right)$ & $1.5 \times 10^{-2}\left(1.9 \times 10^{-2}\right)$ & -10.1 & $3 \times 10^{5}$ \\
\hline PF-S & $1.6 \times 10^{4}$ & $2.6 \times 10^{-4}$ & -14.8 & $2 \times 10^{4}$ \\
\hline PF-O & $4 \times 10^{4}$ & $1.1 \times 10^{-3}$ & -15.0 & $5 \times 10^{4}$ \\
\hline
\end{tabular}

*Maximum mobility is shown in parentheses 
Table 2.7. Performance characteristics of OFET devices with D-A polymer semiconductor on an OTS-18-treatd substrate fabricated and measured.

\begin{tabular}{|c|c|c|c|c|}
\hline \multirow[t]{2}{*}{ Polymer } & \multicolumn{2}{|c|}{ Average Mobility* $\left(\mathrm{cm}^{2} \mathbf{V}^{-1} \mathbf{s}^{-1}\right)$} & \multirow{2}{*}{\multicolumn{2}{|c|}{$\begin{array}{l}V_{\text {th }}(\mathrm{V}) \quad \mathrm{On} / \mathrm{off} \text { ratio } \\
\text { Annealed at } 120{ }^{\circ} \mathrm{C}\end{array}$}} \\
\hline & As fabricated, $60^{\circ} \mathrm{C}$ & Annealed at $120{ }^{\circ} \mathrm{C}$ & & \\
\hline P1 & $1.5 \times 10^{-2}\left(1.7 \times 10^{-2}\right)$ & $2.3 \times 10^{-2}\left(2.4 \times 10^{-2}\right)$ & -12.0 & $10^{5}$ \\
\hline $\mathbf{P 2}$ & $2.1 \times 10^{-2}\left(2.4 \times 10^{-2}\right)$ & $2.4 \times 10^{-2}\left(2.6 \times 10^{-2}\right)$ & -12.0 & $3 \times 10^{5}$ \\
\hline P3 & $0.6 \times 10^{-2}\left(0.7 \times 10^{-2}\right)$ & $1.9 \times 10^{-2}\left(2.0 \times 10^{-2}\right)$ & -7.1 & $3 \times 10^{5}$ \\
\hline
\end{tabular}

*Maximum mobility is shown in parentheses
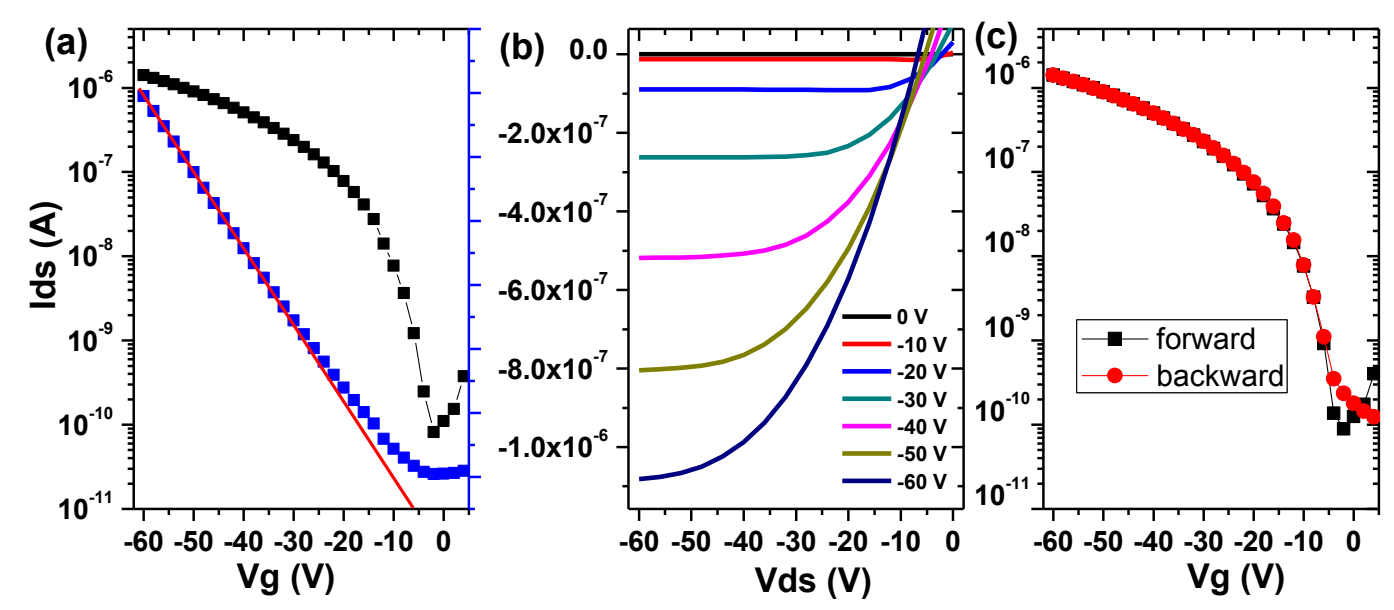

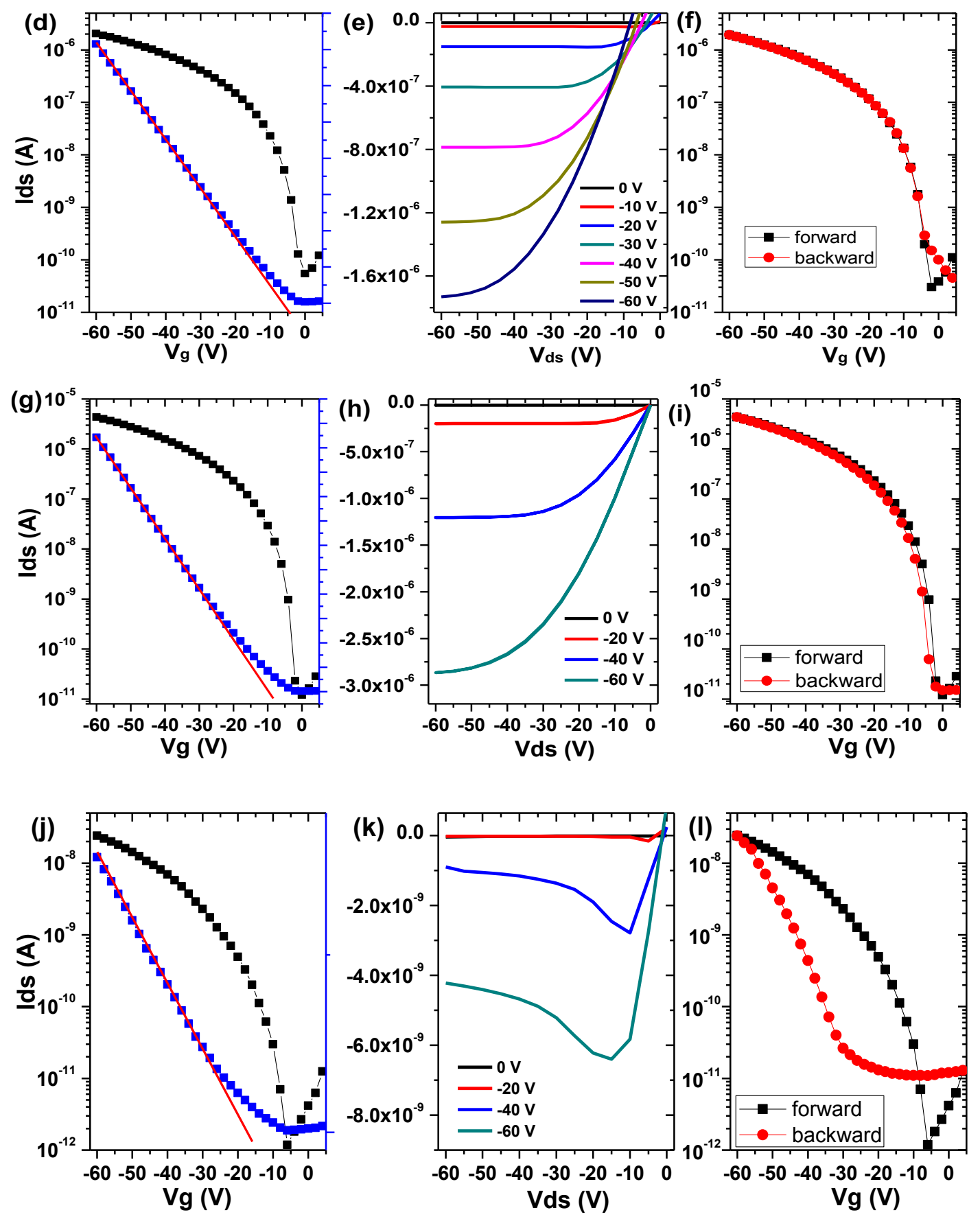



Figure 2.11. Transfer characteristics, output curve, and hysteresis behaviors of OFET devices with D-A polymer channel semiconductors (on OTS-8-modified substrates, annealed at $120^{\circ} \mathrm{C}$ ). P1: (a), (b) and (c), respectively; P2: (d), (e), and (f), P3: (g, h, i), PF-S: (j, k, l), and PF-O: (m, n, o), respectively.





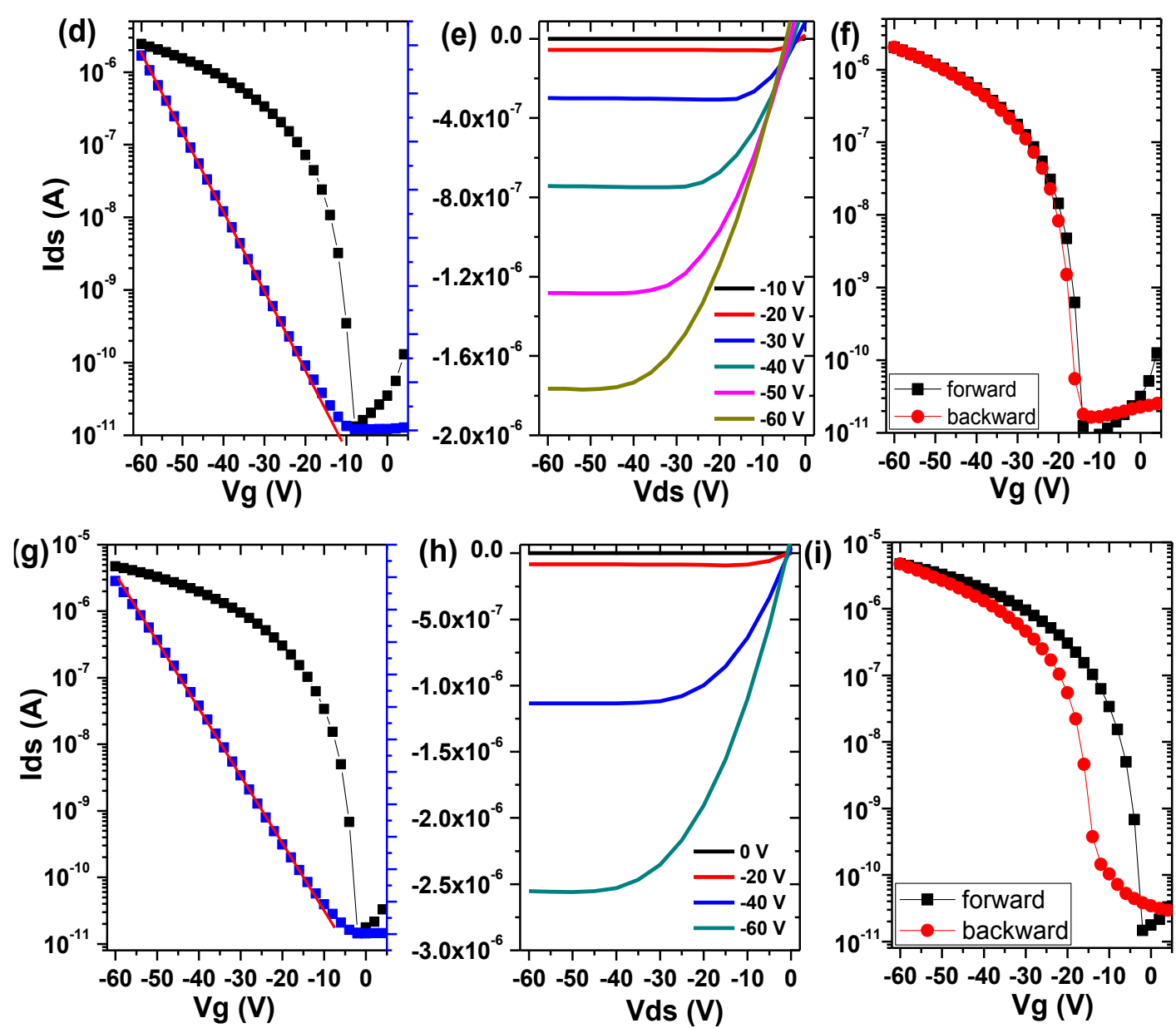

Figure 2.12. Transfer characteristics, output curve, and hysteresis behaviors of OFET devices with D-A polymer channel semiconductors (on OTS-18-modified substrates, annealed at $120^{\circ} \mathrm{C}$ ). P1: (a), (b) and (c), respectively; P2: (d), (e) and (f), respectively; P3: (g, h, i)

A good correlation between the oxidative potentials of the polymers and the threshold voltages of the corresponding devices was noted. For example, the HOMO 
levels of P1, P2, and P3 are higher than those of PF-S and PF-O, thus their threshold voltages $\left(\mathrm{V}_{\text {th }}\right)$ were more positive $(-6 \mathrm{~V}$ for $\mathbf{P 1},-4 \mathrm{~V}$ for $\mathbf{P 2}$ and $-10 \mathrm{~V}$ for $\mathbf{P 3}$ ) as compared with those of PF-S and PF-O, which were more negative $\mathrm{V}_{\text {th }}(-14.8 \mathrm{~V}$ for PF-S and-15 V for PF-O). This was likely related to the hole-injection barrier from the gold contact to the polymer semiconductor as PF-S and PF-O polymers have deeper HOMO levels (-5.82 to $-5.74 \mathrm{eV})$ which are not energetically compatible with gold which has a work function of $-5.1 \mathrm{eV}$. Interestingly P1, P2, and P3 polymers showed very small hysteresis in their transfer characteristics, indicating less trapping in polymer films. ${ }^{204}$

The AFM images of pristine films of P1-3 and PF-S, PF-O spin-cast on $\mathrm{Si} / \mathrm{SiO}_{2}$ substrates from $\mathrm{CB}$ solution at $800 \mathrm{rpm}$ and annealed at $120^{\circ} \mathrm{C}$ are shown in Figures 2.13. P2 formed ordered bicontinuous networks of cylindrical structures, which may contribute to its superior performance among all of the polymers in OFET devices. In contrast to $\mathbf{P 2}$, both $\mathbf{P 1}$ and $\mathbf{P 3}$ formed relatively smooth films without obvious phase separation. Both PF-S and PF-O formed noncontinuous spherical aggregates, consistent with their poor performances (Table 2.6) in OFET devices. Their rootmean-square (RMS) surface roughness values are P1 (1.861 nm), P2 (4.566 nm), P3 (3.504 nm), PF-S (3.929 nm), and PF-O (2.516 nm). In addition, one can see that the exchange of $\mathrm{S} / \mathrm{O}$ atoms did not result in any significant change in film morphology, which is consistent with the results reported by Huang et al. and Shi et al. in similar polymers. ${ }^{181,201}$ 

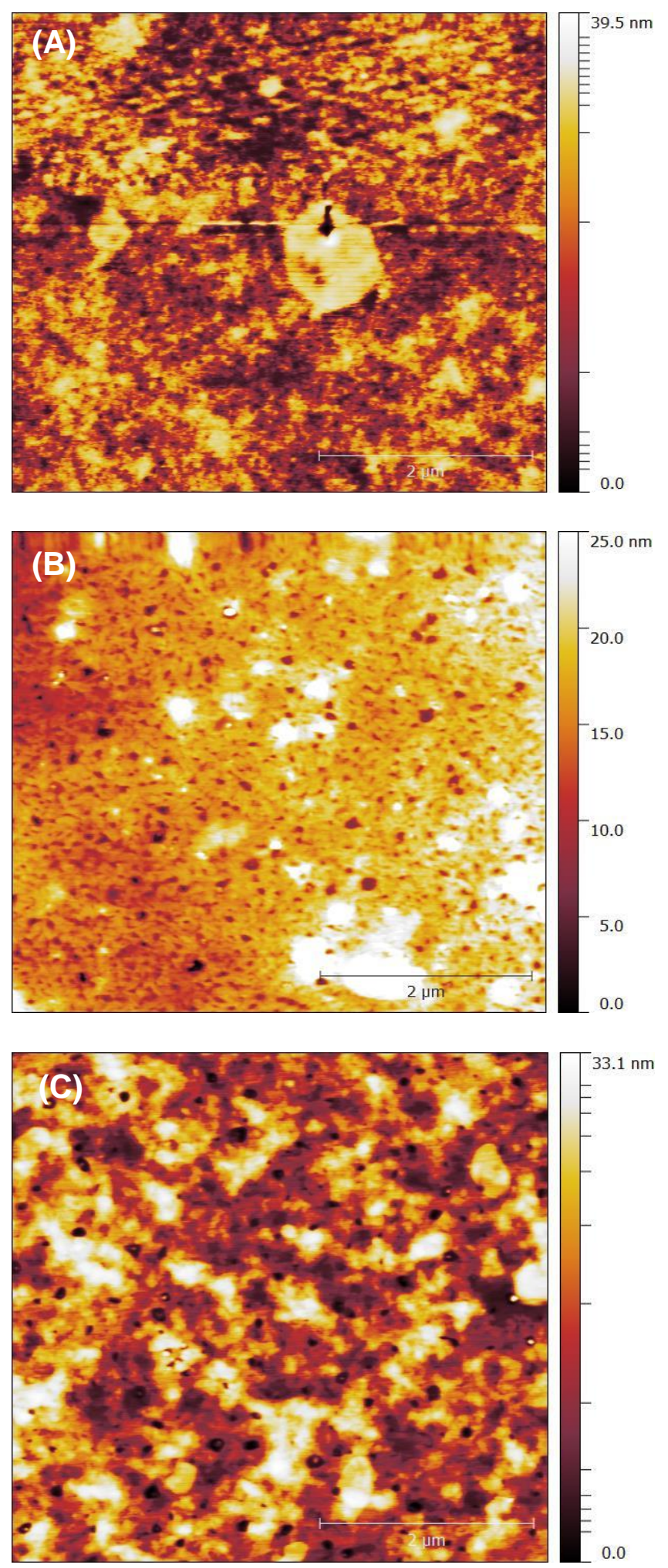

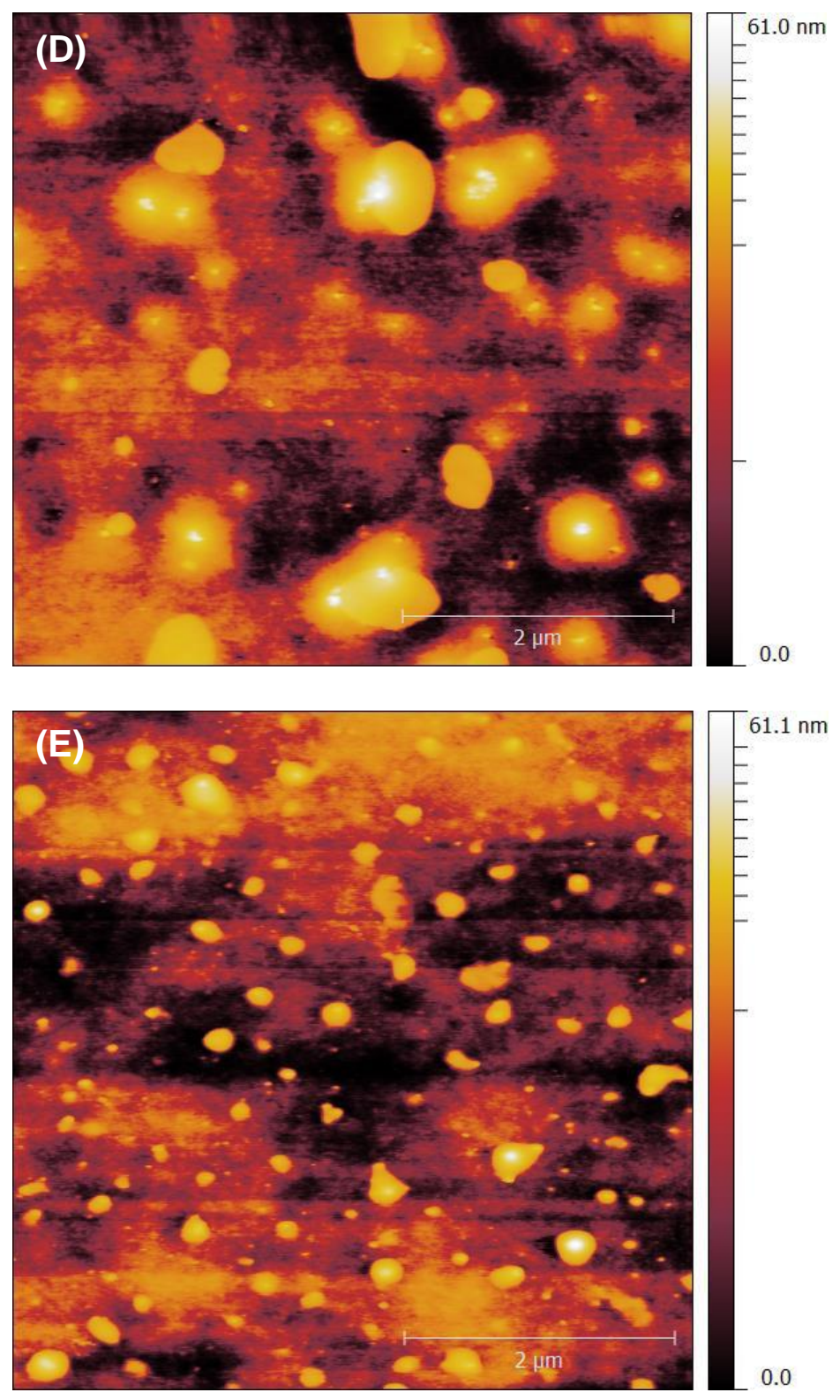

Figure 2.13. Tapping-mode AFM height images $\left(5 \times 5 \mu \mathrm{m}^{2}\right)$ of pristine polymer films on OTS-modified $\mathrm{Si} / \mathrm{SiO}_{2}$ substrates for all polymers, P1 (A), P2 (B), P3(C), PF-S (D), PF-O (E) annealed at $120^{\circ} \mathrm{C}$. 


\subsection{Conclusion}

D-A polymers comprising dithienobenzochalcogenodiazole acceptor units alternating with thienothiophene, bithiophene, and alkylidenefluorene donor units were synthesized for evaluation as semiconductor polymers for OPV and OFET devices. The D-A polymers based on thienothiophene and bithiophene structures have exhibited lower optical bandgaps as compared to their alkylidenefluorene analogs. From solution to thin-film state, all polymers exhibited red shifts in their absorption spectra accompanied with the appearance of shoulder peaks, indicating the formation of more planar polymer backbone orderings or some $J$-aggregates in their solid states. UV-vis and CV studies revealed that oxadiazole-containing polymers displayed deeper LUMO levels and more red-shifted thin-film absorption spectra when compared to their respective thiadiazole-containing counterparts. Among all the polymers, $\mathbf{P} 2$ provided the highest PCE in OPVs and highest mobility and on/off ratio in OFETs. Also worthy of note is the finding that many of these polymer (i.e., P1, P2, and P3) exhibited little hysteresis effects in OFET devices. We believe that the electronic device performance can be further improved through structural and synthesis optimization.

\section{The content in Chapter 2 has been published in}

- Tetrahedron letter DOI: 10.1016/j.tetlet.2014.06.078 and

- Dyes and Pigment DIO:10.1016/j.dyepig.2016.01.03 


\section{CHAPTER 3}

\section{Synthesis and Characterization of $\gamma$-Lactone-}

\section{Pechmann dye Based Donor-Acceptor Conjugated}

\section{Polymers}

\subsection{Introduction}

Conjugated polymer semiconductors have been attracting lots of interest due to their promising applications in various optoelectronic devices such as organic lightemitting diodes (OLEDs), organic photovoltaic (OPVs), and organic field-effect transistors (OFETs). These organic/polymer-based devices have shown advantages such as light weight, solution-processible for low-cost and large-area fabrication, as well as mechanical flexibility and synthetic variety over their inorganic counterparts. ${ }^{104,154,155,205}$ Recent studies have demonstrated that high-performance organic semiconductors could be obtained through a design strategy of alternating an electron donor (D) and an electron acceptor (A) units into the polymer backbone. ${ }^{101,206-209}$ The intramolecular charge transfer in such D-A alternating copolymers provides a means to tune physicoelectrochemical properties of the resulting polymers via carefully choosing individual electron donor and acceptor 
moieties. Therefore, an increased knowledge in choosing an appropriate combination of D/A moieties and also the synthesis of new building blocks plays a pivotal role in the future development of high-performance organic semiconductors. ${ }^{210}$

In comparison to electron donor moieties, the development of electron acceptor moieties has been relatively slow and only a handful of them have been explored. For instance, naphthalene diimide, perylene diimide, benzothiadiazole, benzobisthiadiazole, isoindigo and diketopyrrolopyrrole (DPP) are the commonly utilized electron acceptors moieties for the construction of conjugated D-A polymers..$^{59,133,135,202,210-215}$ Among extensively studied acceptor moieties, DPP and isoindigo both containing $\gamma$-lactam electron withdrawing group have resulted in conjugated D-A polymers with hole mobilities as high as $12 \mathrm{~cm}^{2} \mathrm{~V}^{-1} \mathrm{~s}^{-1} \cdot{ }^{216-218}$ Recently, another acceptor moiety 5,5'-diarylbifuranylidenedione (also called Pechmann dye) also containing a cross-conjugated $\gamma$-lactam electron withdrawing group has emerged as an interesting building block especially for the construction of conjugated D-A polymers for applications in OFETs. ${ }^{139,219,220}$

Pechmann dye was unexpectedly synthesized by Hans von Pechmann in 1882, and then it was fully characterized in 1950s. ${ }^{221-223}$ The development of organic semiconductors has led to several interesting Pechmann dye derivatives substituted with phenyl, thienyl, aminophenyl groups, as well as lactam or sulfur analogs of Pechman dyes by the replacement of endocyclic oxygen in the lactone ring with nitrogen or sulfur. ${ }^{224-229}$ Recently some of these Pechmann dye derivatives have been 
involved in the construction of conjugated polymers. For instance, Zhang and coworkers ${ }^{139,219,220}$ reported high charge-carrier mobility for $\gamma$-lactam -Pechmann dye based small molecules and conjugated polymers. Wang et al. recently reported fused $\delta$-lactone-Pechmann dye based polymers with n-type charge transport characteristics. ${ }^{230}$ However, to the best of our knowledge, conjugated polymers based on $\gamma$-lactone-Pechmann dye as the electron-accepting monomer have not been reported.

This Chapter presents the synthesis and characterization of two new conjugated D-A polymers based on a new acceptor moiety, $\gamma$-lactone-Pechmann dye. $\gamma$-LactonePechmann dye has more interesting properties such as high-planarity, strong electron-accepting nature, and deeper HOMO/LUMO energy levels than its lactam analogs. ${ }^{139,225-227,230}$ These properties were further confirmed by DFT calculations (see Figure 3.1) and consistent with what has been reported by Norsten and coworkers. ${ }^{227}$ For instance, the torsion angle between the flanked thiophene and cross-conjugated lactone core group in $\gamma$-lactone-Pechmann dye is $0^{\circ}$, in contrast to $25^{\circ}$ in its lactam analogs. Such enhanced coplanarity and the more electronegative nature of $\gamma$-lactone-Pechmann dye result in its stronger electron-accepting property than its lactam analogs. Thus, we anticipated that $\gamma$-lactone-Pechmann dye could be a better building block compared to its lactam analogs for the construction of narrowbandgap conjugated D-A polymers. To examine this hypothesis, a brominated 
derivative of bis-3-dodecyl-thiophene flanked $\gamma$-lactone-Pechmann dye as the electron acceptor was polymerized via Stille coupling with two bis-stannyl benzodithiophene derivatives, respectively, as the electron donor. While the resulting two polymers both showed a narrow optical band gap of $1.2-1.3 \mathrm{eV}$, their moderate charge carrier mobility in OFETs devices was probably limited by factors such as the vulnerability against interfacial charge carrier trapping and poor long-range interchain ordering.

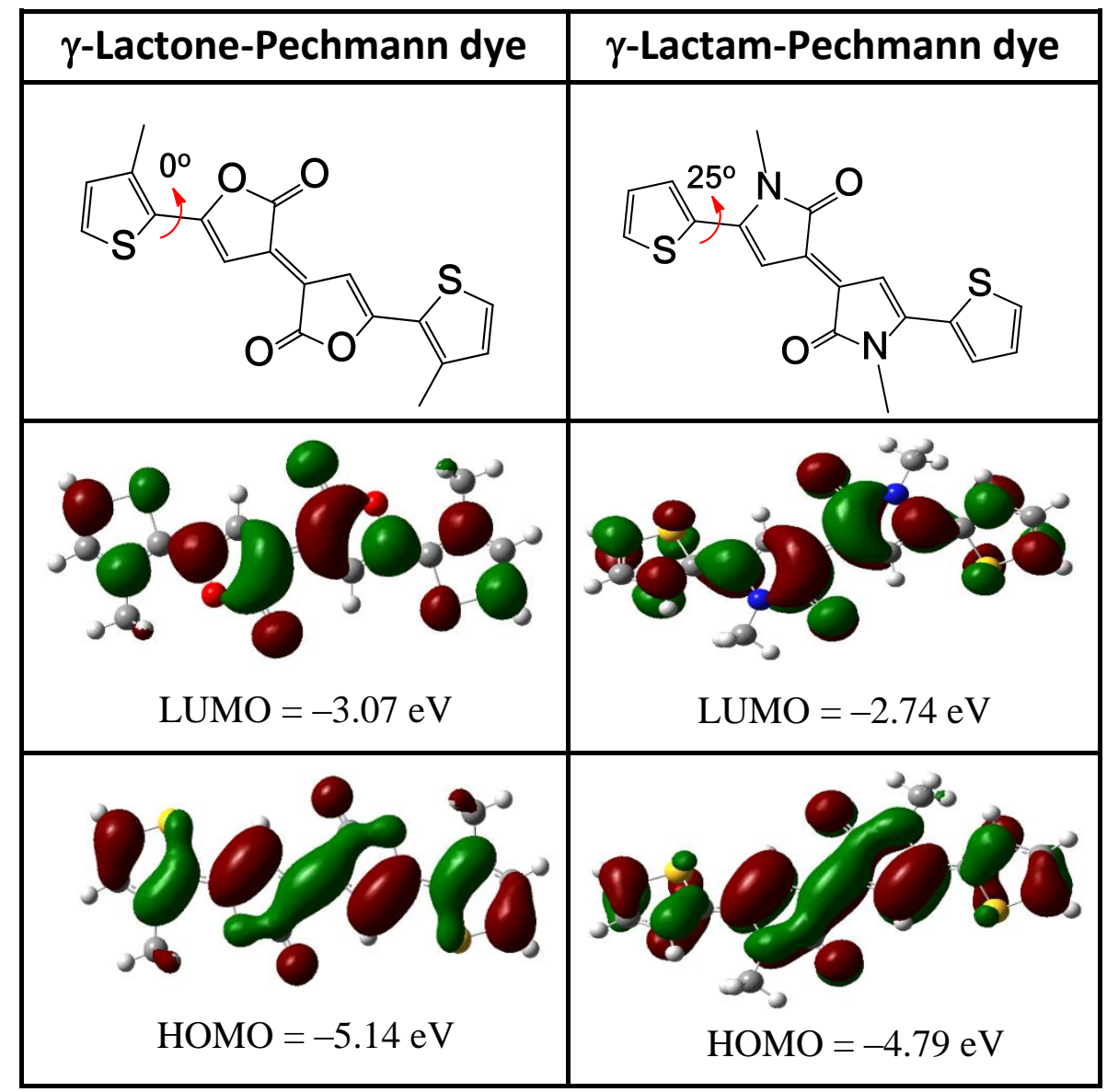

Figure 3.1. DFT calculation at B3LYP/6-31G(d,p) level for geometry, molecular orbital distribution, and HOMO/LUMO energy levels of $\gamma$-lactone-Pechmann dye 
(left) and $\gamma$-lactam-Pechmann dye (right). For computational simplicity, the alkyl chains are replaced with methyl groups.

\subsection{Experimental}

Materials. Unless stated otherwise, starting materials and anhydrous solvents were obtained from Sigma-Aldrich and used without further purification. Compound (17 and $\mathbf{1 8})^{231}$ were prepared according reported procedures. (4,8-Bis(4,5-didecyl thiophen-2-yl)benzo[1,2-b:4,5-b'] dithiophene-2,6-diyl)bis(trimethyl stannane) (2DBDT) and (4,8-bis((2-octyldodecyl)oxy)benzo[1,2-b:4,5-b']dithiophene-2,6-diyl) bis(trimethylstannane) (1D-BDT)were obtained commercially from SunaTech Inc. All the reactions were carried out under inert atmosphere.

\subsubsection{Synthesis of monomers}

\section{(E)-4-(3-dodecylthiophen-2-yl)-4-oxobut-2-enoic acid (17) ${ }^{231}$}

3-dodecylthiophene (3 g, $11.88 \mathrm{mmol})$ and maleic anhydride (1.16 g, 11.88 mmol) were dissolved with stirring in dichloromethane $59 \mathrm{~mL}$ and the mixture cooled in an ice bath. $\mathrm{AlCl}_{3}(3.37 \mathrm{~g}, 25.25 \mathrm{mmol})$ was added portion wise and the mixture stirred at room temperature for $16 \mathrm{~h}$. The mixture was poured into a $10 \%$ $\mathrm{HCl}$ solution containing ice and stirred for $30 \mathrm{~min}$. The organic layer was separated and the aqueous layer was extracted with $10 \mathrm{~mL}(3 \times)$ of dichloromethane and the combined organic layers were washed with water and brine and dried over magnesium sulfate. The solvent was removed and the solid which was triturated with 
hexanes and filtered yielding a light yellow powder which was used without further purification. (Yield: $1.94 \mathrm{~g}, 47 \%$ )

${ }^{1} \mathrm{H}-\mathrm{NMR}\left(300 \mathrm{MHz}, \mathrm{CDCl}_{3}\right): 7.78(\mathrm{~d}, 1 \mathrm{H}), 7.56(\mathrm{~d}, 1 \mathrm{H}), 7.08(\mathrm{~d}, 1 \mathrm{H}), 6.88(\mathrm{~d}$, 1H), $3.06(\mathrm{t}, 2 \mathrm{H}), 1.64(\mathrm{~m}, 2 \mathrm{H}), 1.26(\mathrm{M}, 18 \mathrm{H}), 0.88(\mathrm{t}, 3 \mathrm{H})$.

(E)-5,5'-(3-dodecylthiophen-2-yl)bifuranylidenedione $\quad(\gamma$-lactone-Pechmann dye, Th-Pech) $)^{231}$ (18)

In a $50 \mathrm{~mL}$ round bottom flask was placed Compound $17(2.41 \mathrm{~g}, 6.87 \mathrm{mmol})$, $\mathrm{CuCl}(0.2 \mathrm{~g}, 2.06 \mathrm{mmol})$ and ammonium chloride $(0.26 \mathrm{~g}, 4.8 \mathrm{mmol})$ in $18 \mathrm{~mL}$ of acetic anhydride. The mixture was refluxed for $2 \mathrm{~h}$ and its color was turned to dark purple. After cooling to room temperature, the solid was collected by vacuum filtration and washed with acetic acid and ethanol successively. The dark solid dissolved in chloroform and filtered to remove the insoluble copper salts yielding a purple solid after concentration $(1.56 \mathrm{~g}, 34 \%)$.

${ }^{1} \mathrm{H}-\mathrm{NMR}\left(300 \mathrm{MHz}, \mathrm{CDCl}_{3}\right): 7.53$ (d, 2H), $7.28(\mathrm{~S}, 2 \mathrm{H}), 7.07$ (d, 2H), 2.93 (t, 4H), $1.73(\mathrm{~m}, 4 \mathrm{H}), 1.29(\mathrm{M}, 36 \mathrm{H}), 0.88(\mathrm{t}, 6 \mathrm{H})$.

(E)-5,5'-bis(5-bromo-3-dodecylthiophen-2-yl)-2H,2'H-[3,3'-bifuranylidene]2,2'-dione (Br2-Th-Pech) (19)

$\mathrm{N}$-Bromosuccinimide $(56 \mathrm{mg}, 0.315 \mathrm{mmol}$ ) was added in two portions to the solution of Compound $18(0.1 \mathrm{~g}, 0.15 \mathrm{mmol})$ in $10 \mathrm{~mL}$ of $\mathrm{CH}_{2} \mathrm{Cl}_{2}$ at $0{ }^{\circ} \mathrm{C}$. The reaction mixture was stirred in the dark for $24 \mathrm{~h}$ at room temperature under nitrogen atmosphere. Then, water $(30 \mathrm{~mL})$ was added and extracted by $\mathrm{CH}_{2} \mathrm{Cl}_{2}(3 \times 15 \mathrm{~mL})$. 
The combined organic extract was washed with brine $(2 \times 50 \mathrm{~mL})$, dried over anhydrous $\mathrm{Na}_{2} \mathrm{SO}_{4}$ and concentrated under reduced pressure after filtration. The residue was purified by column chromatography with hexane and $\mathrm{CH}_{2} \mathrm{Cl}_{2}(1: 0.25$, v/v) as the eluent. Compound 19 was obtained as dark purple solids. Which was further purified by recrystallization from ethanol (Yield: $0.12 \mathrm{~g}, 24 \%$ ).

${ }^{1} \mathrm{H}$ NMR $\left(300 \mathrm{MHz}, \mathrm{CDCl}_{3}\right) \delta(\mathrm{ppm}): 7.21(\mathrm{~s}, 2 \mathrm{H}), 7.00(\mathrm{~s}, 2 \mathrm{H}), 2.86-2.81(\mathrm{t}$, 4H), 1.69-1.67 (m, 4H), 1.56-1.26 (m, 36H), 0.87 (m, 6H).

\subsubsection{Synthesis of polymer P5 and P6}

\section{Synthesis of P5}

To a $25 \mathrm{~mL}$ round-bottom flask Compound 19 (85 mg, $0.103 \mathrm{mmol}),(4,8-$ bis(4,5-didecylthiophen-2-yl)benzo[1,2-b:4,5-b']dithiophene-2,6-diyl)bis(trimethyl stannane) (128.22 mg, $0.103 \mathrm{mmol})$, tris(dibenzylideneacetone) dipalladium (0) $\left(\mathrm{Pd}_{2}(\mathrm{dba})_{3}\right)(2 \mathrm{~mol} \%, 2.14 \mathrm{mg})$, and tri $(o$-tolyl $)$ phosphine $\left(\mathrm{P}(\mathrm{o}-\mathrm{tol})_{3}\right)(8.2 \mathrm{~mol} \%, 3$ $\mathrm{mg}$ ) were added under argon atmosphere. After three cycles of degassing and purging with argon, $10 \mathrm{~mL}$ of anhydrous toluene was added. The flask was charged with argon after three cycles of freeze-pump-thaw. The mixture was stirred for $24 \mathrm{~h}$ at 110 ${ }^{\circ} \mathrm{C}$ under argon. After being cooled to room temperature, the reaction mixture was poured into $100 \mathrm{~mL}$ of methanol and filtered. The resulting crude product was then subjected to Soxhlet extraction with methanol, acetone, hexane, and chloroform in 
sequence. The n-hexane fraction was evaporated to dryness and reprecipitated from methanol to give $\mathbf{P 5}$ as a black powder (Yield: $128 \mathrm{mg}, 78.5 \%$ ).

${ }^{1} \mathrm{H}$ NMR (300 MHz, $\left.\mathrm{C}_{2} \mathrm{D}_{2} \mathrm{Cl}_{4}\right) \delta(\mathrm{ppm}): 7.71(\mathrm{br}, 2 \mathrm{H}), 7.54-7.38(\mathrm{br}, 2 \mathrm{H}), 7.17-$ 7.12 (br, 2H), 6.89 (br, 2H), 2.82-2.59 (d, 12H), 1.67 (br, 4H), 1.51-0.90 (m, 100H), $0.79(\mathrm{~m}, 18 \mathrm{H}) . M_{\mathrm{n}}=7.7 \mathrm{kDa}, M_{\mathrm{w}}=12.3 \mathrm{kDa}, \mathrm{PDI}=1.6$.

\section{Synthesis of P6}

To a $25 \mathrm{~mL}$ round-bottom flask Compound 19 (74.8 mg, $0.091 \mathrm{mmol}),(4,8$ bis((2-octyldodecyl)oxy)benzo[1,2-b:4,5-b']dithiophene-2,6-diyl)bis(trimethyl stannane) (100.81 mg, $0.091 \mathrm{mmol})$, tris(dibenzylideneacetone)dipalladium (0) $\left(\mathrm{Pd}_{2}(\mathrm{dba})_{3}\right)(2 \mathrm{~mol} \%, 1.9 \mathrm{mg})$, and tri $(o$-tolyl $)$ phosphine $\left(\mathrm{P}(\mathrm{o}-\mathrm{tol})_{3}\right)(8.2 \mathrm{~mol} \%, 2.27$ mg) were added under argon atmosphere. After three cycles of degassing and purging with argon, $9 \mathrm{~mL}$ of anhydrous toluene was added, then the flask was charged with argon after three cycles of freeze-pump-thaw. The mixture was stirred for $24 \mathrm{~h}$ at 90 ${ }^{\circ} \mathrm{C}$ under argon. After being cooled to room temperature, the reaction mixture was poured into $100 \mathrm{~mL}$ of methanol and filtered. The resulting crude product was subjected to Soxhlet extraction with methanol, acetone, hexane, and chloroform in sequence. The chloroform fraction was evaporated to dryness and reprecipitated from methanol to give $\mathbf{P 6}$ as a black powder (Yield: $112.8 \mathrm{mg}, 86 \%$ ).

${ }^{1} \mathrm{H}$ NMR (300 MHz, $\left.\mathrm{C}_{2} \mathrm{D}_{2} \mathrm{Cl}_{4}\right) \delta(\mathrm{ppm}):$ 7.70-6.50 (br, 6H), 4.18 (br, 4H), 2.84 (br, 4H), $1.84(\mathrm{br}, 2 \mathrm{H}), 1.9-0.50(\mathrm{~m}, 126 \mathrm{H}) . M_{\mathrm{n}}=12 \mathrm{kDa}, M_{\mathrm{w}}=23 \mathrm{kDa}, \mathrm{PDI}=1.9$. 


\subsubsection{GPC traces for polymers and NMR spectra}

\subsubsection{GPC traces for polymers}
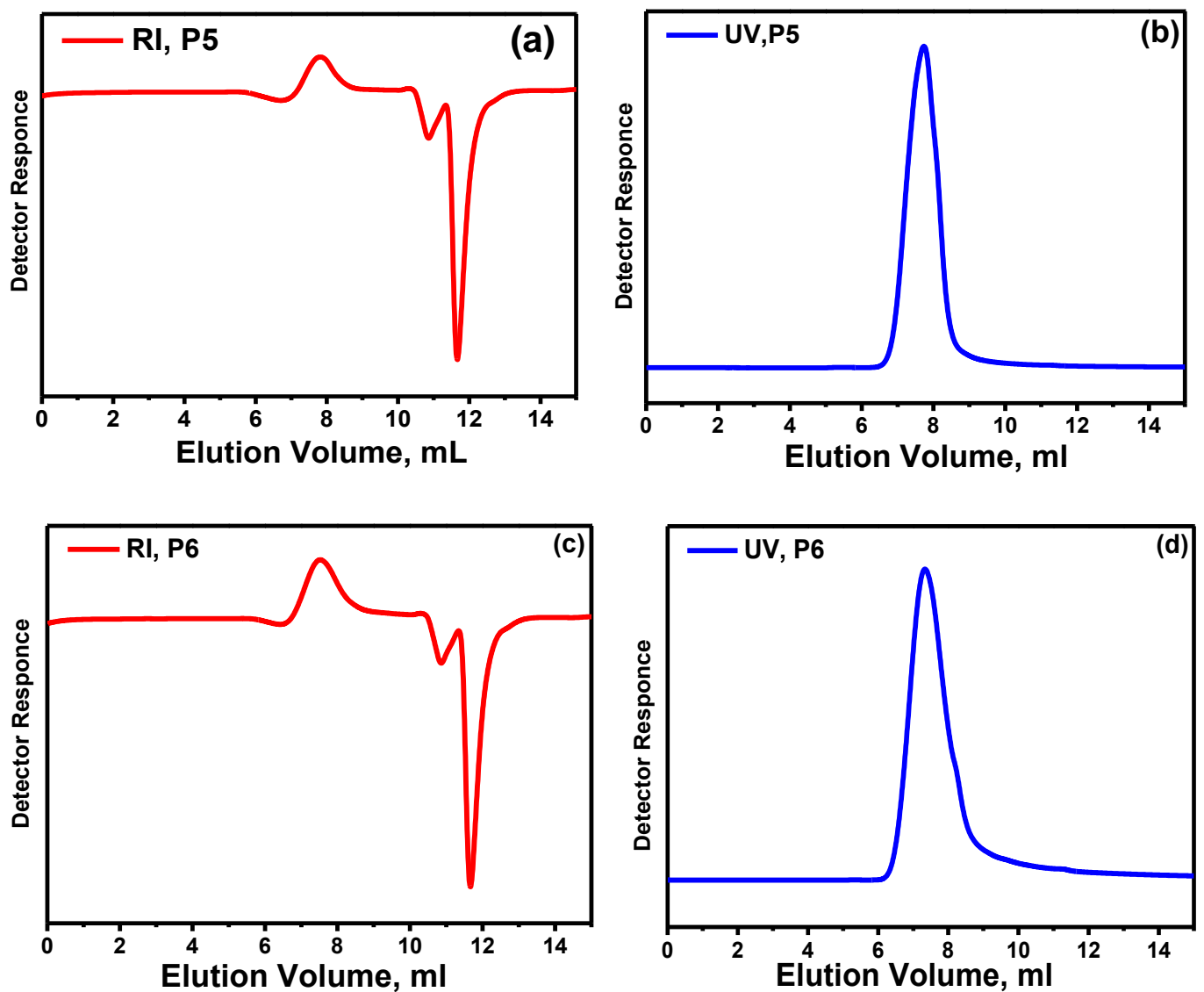

Figure 3.2. GPC traces for $\mathbf{P 5}$ and $\mathbf{P 6}$ (using $\mathrm{CHCl}_{3}$ as eluent at $80{ }^{\circ} \mathrm{C}$ ) from $\mathrm{RI}(\mathrm{a}$ and c) and UV (550 nm) detectors (b and d), respectively. 


\subsubsection{NMR spectra of monomers and polymers}
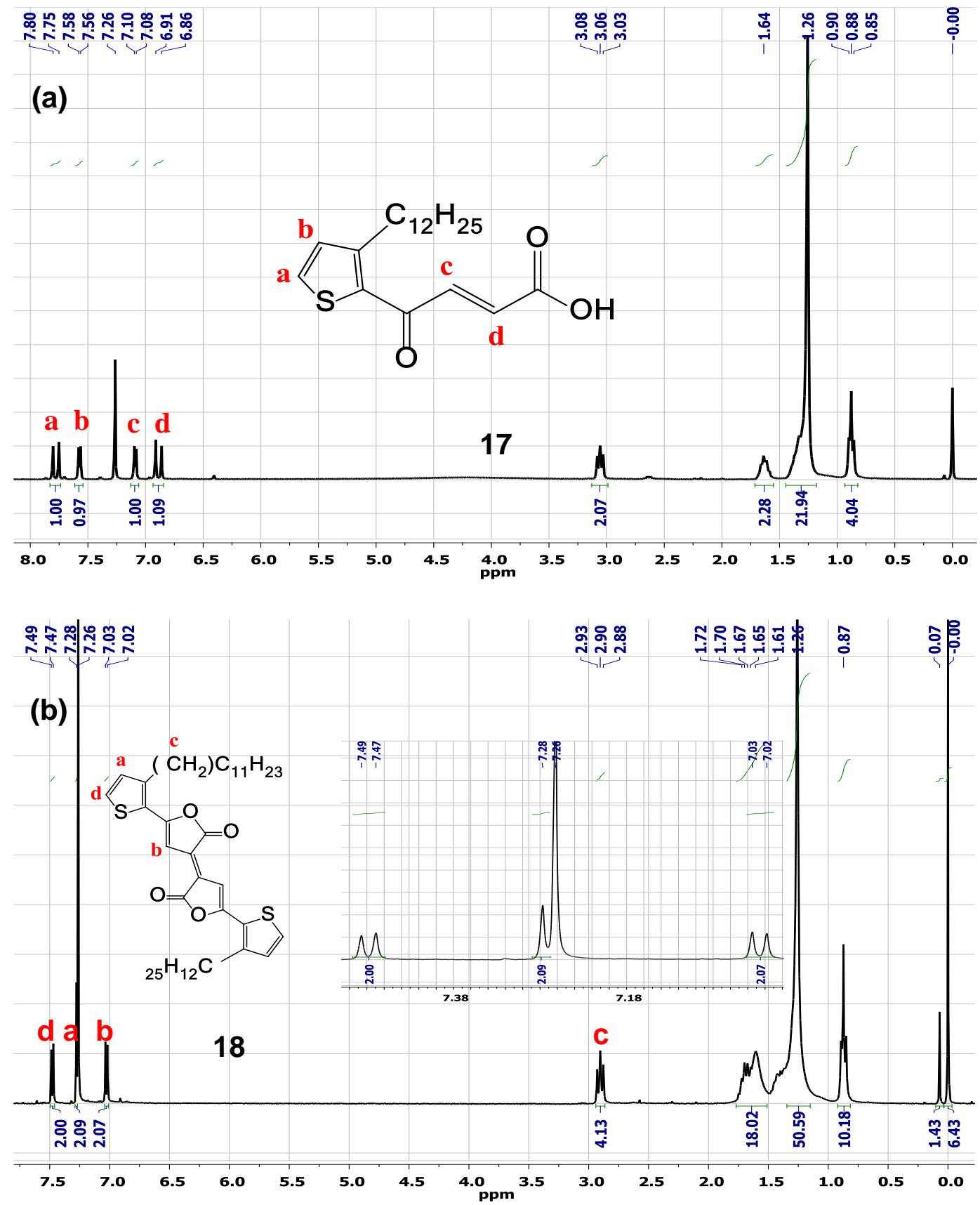

Figure 3.3. ${ }^{1} \mathrm{H}$ NMR (300 MHz) spectra for compound 17 and 18 (a and b), respectively, in $\mathrm{CDCl}_{3}$ solvent at R.T. 

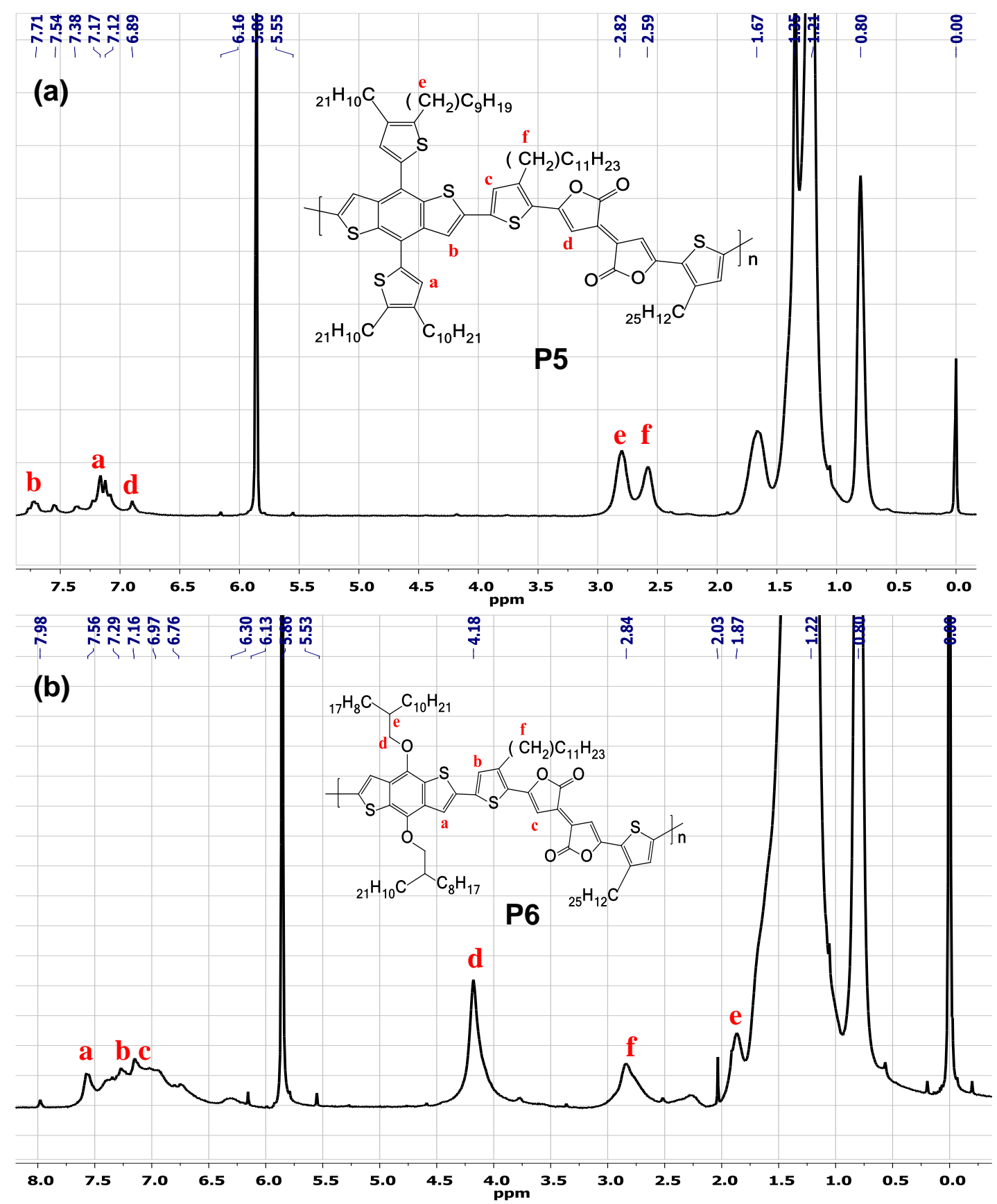

Figure 3.4. ${ }^{1} \mathrm{H}$ NMR (300 MHz) spectra for $\mathbf{P 5}$ (a) and $\mathbf{P 6}$ (b) in $\mathrm{C}_{2} \mathrm{D}_{2} \mathrm{Cl}_{4}$ solvent at $100{ }^{\circ} \mathrm{C}$. 


\subsection{Results and Discussion}

\subsubsection{Synthesis of monomers and polymers}

As depicted in Scheme 6, Compound $\mathbf{1 7} \& \mathbf{1 8}$ was synthesized according to the reported procedure, ${ }^{227}$ where $\beta$-aroylacrylic acid or (E)-4-oxo-4-(2-thienyl)-2butenoic acids were obtained via Friedel-Crafts acylation of maleic anhydride with 3-dodecylthiophene to give Compound $\mathbf{1 7}$ in $47 \%$ yield. In the presence of cuprous chloride catalyst and acetic anhydride solvent, $\beta$-aroylacrylic acid was dimerized to give $\gamma$-lactone-Pechmann dye, Compound $\mathbf{1 8}$ in $34 \%$ yield. The bromination of Compound 18 with N-bromosuccinimide (NBS) followed by purification via column chromatography and recrystallization gave Compound 19 in $24 \%$ yield. Copolymers P5 and P6 were synthesized via Stille polycondensation of an acceptor moiety, Compound 19, with (4,8-bis(4,5-didecylthiophen-2-yl)benzo[1,2-b:4,5-b'] dithiophene-2,6-diyl)bis(trimethylstannane) (2D-BDT) and (4,8-bis((2-octyl dodecyl)oxy)benzo-[1,2-b:4,5-b']dithiophene-2,6-diyl)bis(trimethylstannane) (1DBDT), respectively, by refluxing at $110^{\circ} \mathrm{C}$ for $24 \mathrm{~h}$ in the presence of $\mathrm{Pd}_{2}(\mathrm{dba})_{3} / \mathrm{P}(\mathrm{o}-$ tol) $)_{3}$ catalyst and dry toluene as the solvent (Scheme 7). Finally, all the copolymers were purified via precipitation from methanol, followed by Soxhlet extraction using methanol, acetone, hexane, and chloroform in sequence. The polymers collected from n-hexane fraction in the yield of 78.5\% for $\mathbf{P 5}$ and $86 \%$ for $\mathbf{P 6}$ as collected from chloroform fraction. The details of synthesis and characterization are described in the experimental section. Both $\mathbf{P 5}$ and $\mathbf{P 6}$ polymers show relatively good solubility 
in solvents such as chloroform and mono/dichlorobenzene. We note that when the alkyl chain in the 1D-BDT unit of P6 was replaced with ethylhexyl group, the polymer was found insoluble in all chlorinated solvents, probably due to strong intermolecular aggregation. Therefore, its electrochemical and device properties were not studied further. The molecular weights of the P5 and P6 polymers were evaluated by gel permeation chromatography (GPC) at $80{ }^{\circ} \mathrm{C}$ with $\mathrm{CHCl}_{3}$ as the eluent (see Section 3.4.3). The number-average molecular weight $\left(\mathrm{M}_{\mathrm{n}}\right)$ /polydispersity index (PDI) of $\mathbf{P 5}$ and $\mathbf{P 6}$ is $7.7 \mathrm{kDa} / 1.6$ and $12 \mathrm{kDa} / 1.9$, respectively.

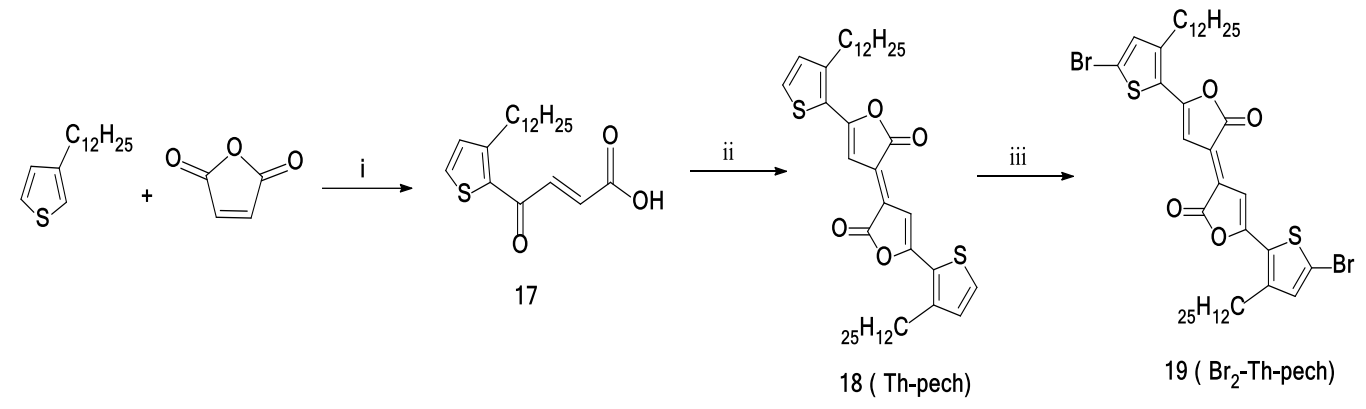

Scheme 6: Synthetic route to Compound 19 (Br2-Th-Pech). (i) $\mathrm{AlCl}_{3}, \mathrm{CH}_{2} \mathrm{Cl}_{2}$, R.T., $18 \mathrm{~h}, 47 \%$ yield. (ii) $\mathrm{CuCl}, \mathrm{NH}_{4} \mathrm{Cl}, \mathrm{Ac}_{2} \mathrm{O}, 140{ }^{\circ} \mathrm{C}, 2 \mathrm{~h}, 34 \%$ yield. (iii) $\mathrm{NBS}, \mathrm{CH}_{2} \mathrm{Cl}_{2}$, R.T., 24 h, 24\% yield. 

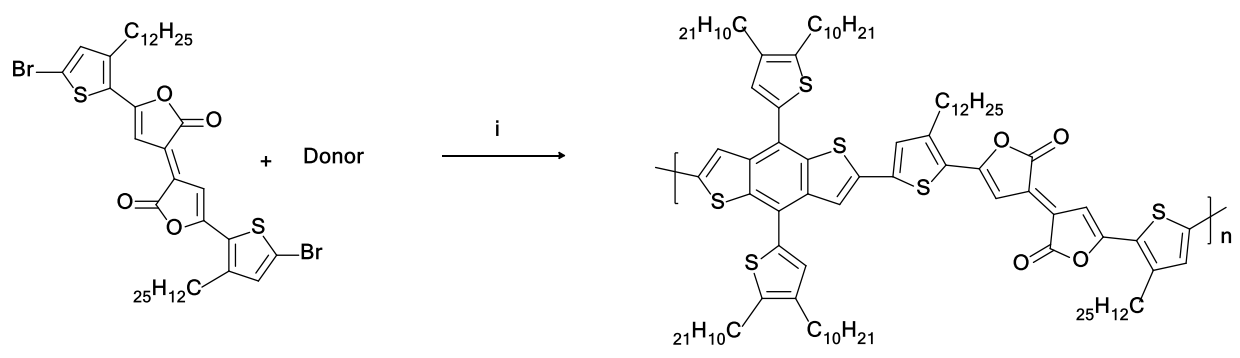

P5
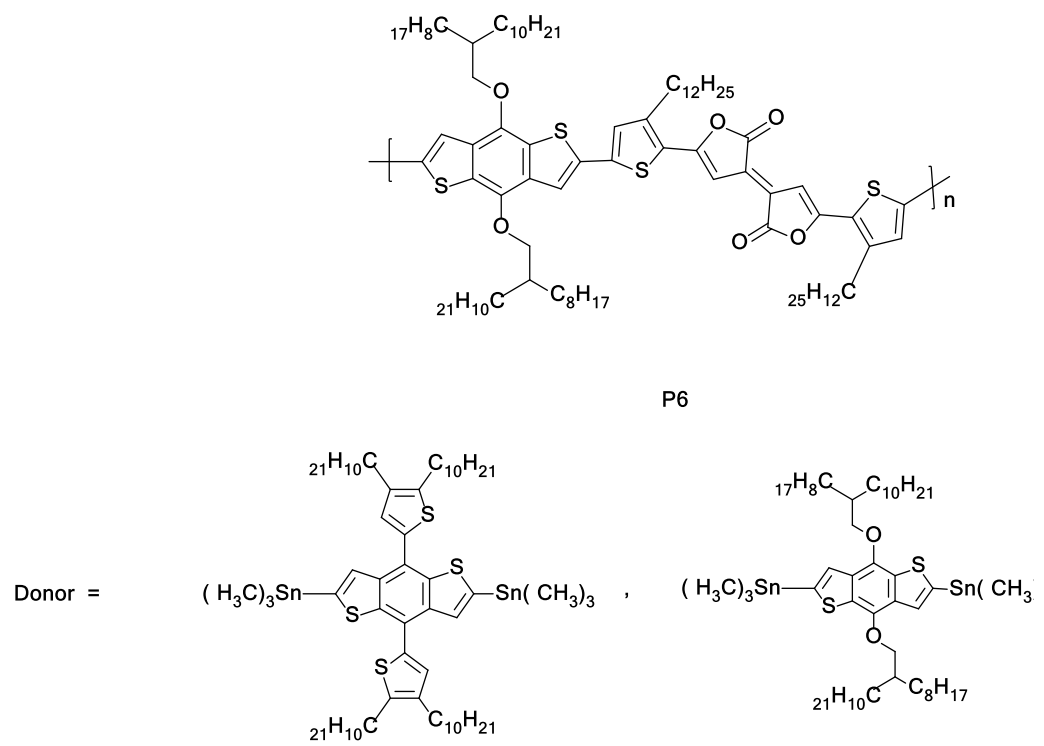

Scheme 7: Synthetic route to $\mathbf{P 5}$ and $\mathbf{P 6}$ polymers via Stille coupling. (i) $\mathrm{Pd}_{2}(\mathrm{dba})_{3}$, $\mathrm{P}(\mathrm{o}-\text { tol })_{3}$, toluene, $24 \mathrm{~h}, 110{ }^{\circ} \mathrm{C}, 78.8 \%(\mathrm{P5})$ and $90{ }^{\circ} \mathrm{C}, 86 \%$ (P6) yield.

\subsubsection{Thermal Properties}

The thermal properties of both P5 and P6 polymers were investigated by thermogravimetric (TGA) and differential scanning calorimeter (DSC) analysis at a heating rate of $10{ }^{\circ} \mathrm{C} \mathrm{min}-1$ under a nitrogen atmosphere. Figure 3.5 (a) shows onset decomposition temperature $\left(T_{\mathrm{d}}\right)$ at $5 \%$ weight loss is $323{ }^{\circ} \mathrm{C}$ for $\mathbf{P 5}$ and $305{ }^{\circ} \mathrm{C}$ for P6, which indicates that both polymers have sufficient stability for use in 
optoelectronic devices. Figure 3.5 (b) presents the DSC analysis for both P5 and P6 polymers, where neither the melting point $\left(T_{\mathrm{m}}\right)$ nor the glass transition temperature $\left(T_{\mathrm{g}}\right)$ could be observed. This result indicates that both polymers have amorphous characteristics.
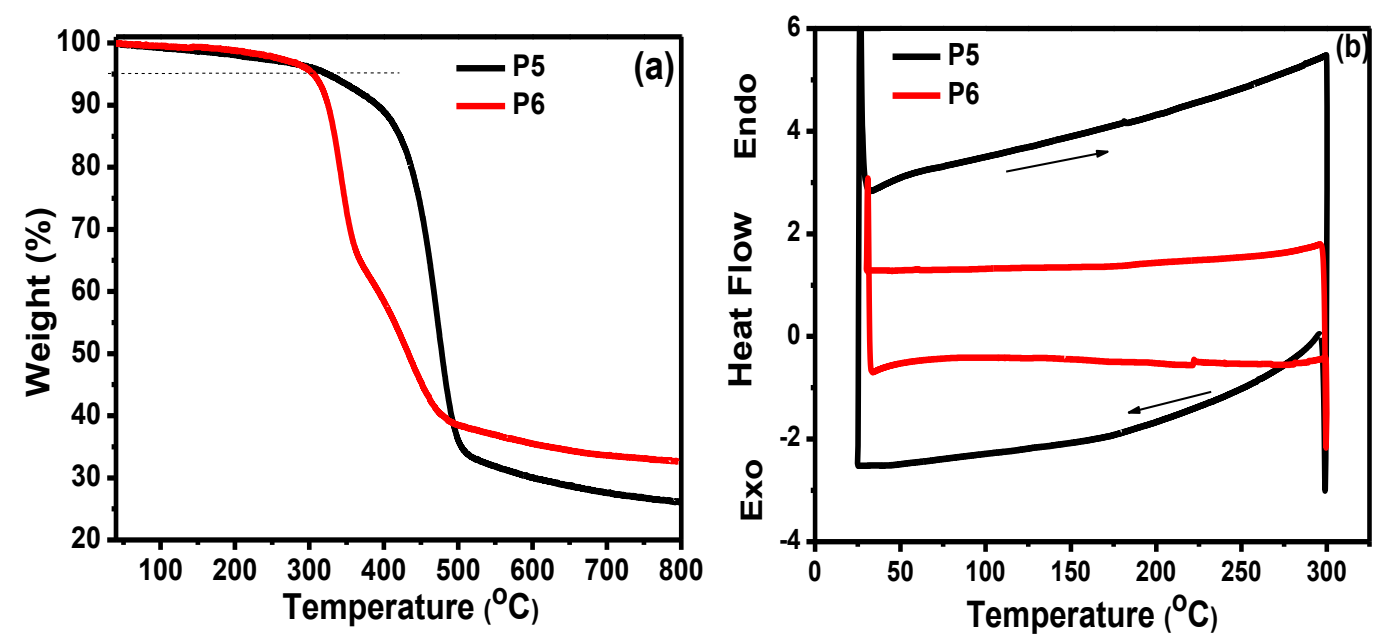

Figure 3.5. (a) TGA and (b) DSC traces at a heating rate of $10{ }^{\circ} \mathrm{C} \min ^{-1}$ under nitrogen.

\subsubsection{Optical Properties}

As shown in Figure 3.6, the optical properties of both $\mathbf{P 5}$ and $\mathbf{P 6}$ polymers were investigated using UV-vis-NIR spectra collected from their chloroform solutions and thin films. The optical data including the maximum absorption peak wavelength $\left(\lambda_{\max }\right)$, onset absorption wavelengths ( $\left.\lambda_{\text {onset }}\right)$, and the corresponding optical band gaps $\left(E_{\mathrm{g}}{ }^{\mathrm{opt}}\right)$ are summarized in Table 3.2. Both solution and thin-film spectra for $\mathbf{P 5}$ and P6 exhibit a broad absorption band extending to NIR region up to $1000 \mathrm{~nm}$. The 
absorption spectra for both polymers present two distinct peaks localized at 350-550 $\mathrm{nm}$ typical of a $\pi-\pi^{*}$ transition and at $550-1000 \mathrm{~nm}$ related to an intramolecular charge transfer (ICT). In addition, the absorption bands of $\mathbf{P 5}$ in both solution and in films show more vibronic features compared to those of $\mathbf{P 6}$ in which both absorption bands are featureless. As compared to absorption spectra in solution, the thin film spectra at $\lambda_{\text {onset }}$ for both $\mathbf{P 5}$ and $\mathbf{P 6}$ polymers are red shifted by ca.10 and $25 \mathrm{~nm}$, respectively. While there was no obvious shift of the $\lambda_{\max , a b s}$ for both polymers in films vs. in solution, the thin film of $\mathbf{P 5}$ showed a slight enhancement in the absorption peak at $818 \mathrm{~nm}$, accompanied by a slight decrease of the absorbance of the shoulder peak at $760 \mathrm{~nm}$. In contrast to $\mathbf{P 5}$, the normalized absorption spectrum of $\mathbf{P 6}$ in thin films overlaps well with its absorption spectrum in chloroform.

In order to investigate whether there is any intermolecular aggregation in solution, we characterized the temperature dependence of the UV-vis-NIR absorption for both $\mathbf{P 5}$ and $\mathbf{P 6}$ in chlorobenzene (CB) at $5 \times 10^{-5} \mathrm{M}$. The experimental results (Figure 3.6 (c) \& (d)) show an obvious enhancement of the absorbance for the vibronic peak at $807 \mathrm{~nm}$ when the solution of $\mathbf{P 5}$ was cooled from $100{ }^{\circ} \mathrm{C}$ to room temperature, in contrast to the minor red-shift in the absorption spectrum of $\mathbf{P 6}$ in the same cooling process. These results suggest that there might be some extent of intermolecular aggregation in the solution state of $\mathbf{P 5}$ at room temperature. But for P6, it remains unclear whether there was little intermolecular aggregation in $\mathrm{CB}$ at room temperature, or the aggregates were too stable to break upon heating up to 100 
${ }^{\circ} \mathrm{C}$. The results above suggest better planarity and rigidity of $\mathbf{P 6}$ compared to those of P5, which results in a less conformational change of $\mathbf{P 6}$ from solution to film states. $^{230,232}$ Such difference might be attributed to the twist between the pendant alkylthiophene and the conjugated backbone in P5, which is discussed further below. Calculation based on thin film onset absorptions spectra gives an optical bandgap of $1.29 \mathrm{eV}$ for $\mathbf{P 5}$ and $1.22 \mathrm{eV}$ for $\mathbf{P 6}$. The change in $E_{\mathrm{g}}{ }^{\text {opt }}$ between the two polymers is mainly attributed to the difference in the chemical structure of the donor moieties i.e., 2D-BDT and 1D-BDT. P5 shows slightly lower HOMO level (-5.25 $\mathrm{eV})$ than $\mathbf{P 6}(-5.22 \mathrm{eV})$, which is ascribed to the weaker electron-donating ability of the twisted thiophene appended from the BDT unit of P5 compared to that of the alkoxyl group in P6. Therefore, the optical band gap of P5 is slightly larger than that of P6. ${ }^{233,234}$


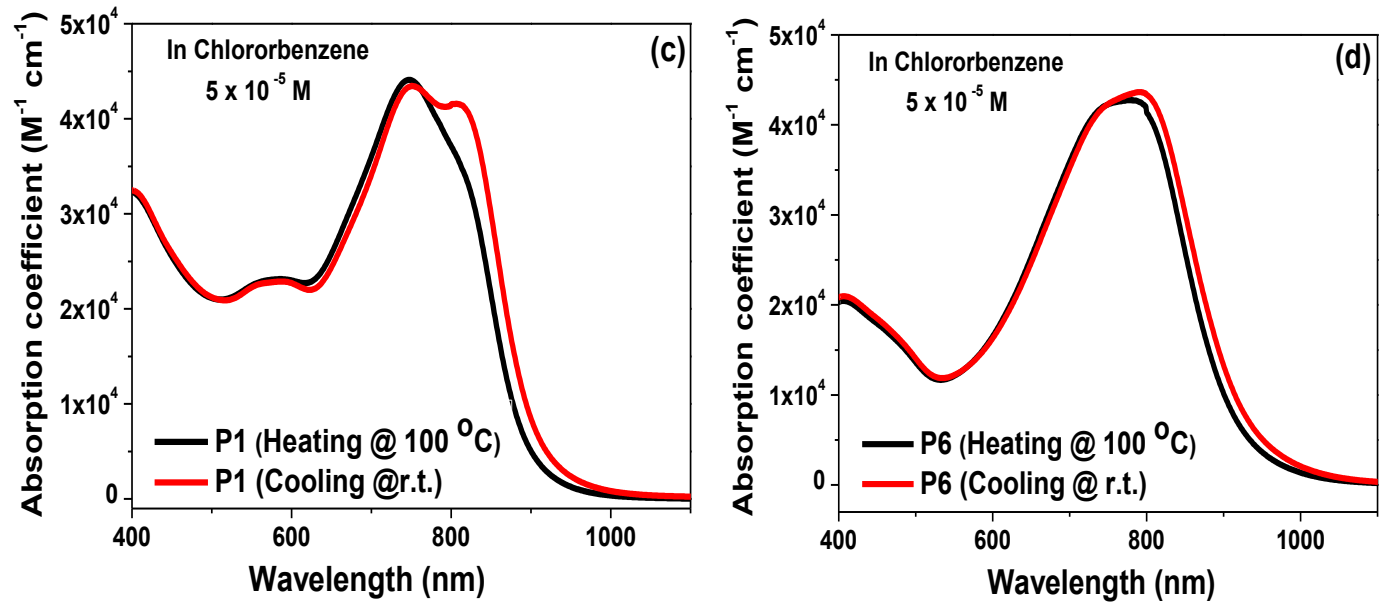

Figure 3.6. UV-vis-NIR absorption spectra of polymers in chloroform and in thin films measured at room temperature (a) for P5 and (b) for P6. Temperature dependent absorption spectra of polymers in chlorobenzene solution (c) for P5 and (d) for $\mathbf{P 6}$.

Table 3.1. Optical properties of P5 and P6 polymers.

\begin{tabular}{|c|c|c|c|c|c|c|c|}
\hline \multirow[b]{2}{*}{ Polymer } & \multicolumn{2}{|c|}{ Molecular weight } & \multicolumn{2}{|c|}{ In $\mathrm{CHCl}_{3}$} & \multicolumn{2}{|c|}{ Film } & \multirow[b]{2}{*}{$\begin{array}{r}{ }^{\mathrm{c}} \boldsymbol{E}_{\mathrm{g}}{ }^{\mathrm{pp}} \\
(\mathrm{eV})\end{array}$} \\
\hline & $\begin{array}{c}M_{\mathbf{n}} \\
{[\mathrm{kDa}]}\end{array}$ & $M_{\mathrm{w}} / M_{\mathrm{n}}$ & $\begin{array}{l}\mathbf{a}_{\max } \\
{[\mathrm{nm}]}\end{array}$ & $\begin{array}{l}{ }^{\mathbf{b}} \lambda_{\text {onset }} \\
{[\mathrm{nm}]}\end{array}$ & $\begin{array}{l}\mathbf{a}_{\max } \\
{[\mathrm{nm}]}\end{array}$ & $\begin{array}{c}{ }^{\mathbf{b}} \lambda_{\text {onset }} \\
{[\mathrm{nm}]}\end{array}$ & \\
\hline P5 & 7.7 & 1.6 & 817 & 953 & 818 & 963 & 1.29 \\
\hline P6 & 12 & 1.9 & 794 & 995 & 800 & 1020 & 1.22 \\
\hline
\end{tabular}

${ }^{\mathrm{a}}$ Absorption maxima. ${ }^{\mathrm{b}}$ The onset absorption. ${ }^{\mathrm{c}}$ Estimated from the onset absorption in films $\left(\lambda_{\text {onset }}\right): E_{\mathrm{g}}^{\text {opt }}(\mathrm{eV})=1240 / \lambda_{\text {onset }}[\mathrm{nm}]$ 


\subsubsection{Electrochemical Properties and DFT Calculation}

The sample films for electrochemical measurements were coated on the surface of the glassy carbon electrode. The $\mathrm{CV}$ curves were calibrated using the ferrocene/ferrocenium $\left(\mathrm{Fc} / \mathrm{Fc}^{+}\right)$redox couple as an external standard which was measured under the same condition after the measurement of samples. The energy level of $\mathrm{Fc} / \mathrm{Fc}^{+}$was assumed at $-4.8 \mathrm{eV}$ to vacuum. ${ }^{235}$ The half-wave potential of the ferrocene/ferrocenium $\left(\mathrm{Fc} / \mathrm{Fc}^{+}\right)$redox couple $\left(\mathrm{E}_{1 / 2}, \mathrm{Fc}, \mathrm{Fc}^{+}\right)$was estimated from $\mathrm{E}_{1 / 2}$, $\mathrm{Fc}, \mathrm{Fc}^{+}=\left(\mathrm{E}_{\mathrm{ap}}+\mathrm{E}_{\mathrm{cp}}\right) / 2$, where $\mathrm{E}_{\mathrm{ap}}$ and $\mathrm{E}_{\mathrm{cp}}$ are the anodic and cathodic peak potentials, respectively. As indicated on Figure 3.7 (b), the half-wave potential of $\mathrm{Fc} / \mathrm{Fc}^{+}$is to be $\left[\mathrm{E}_{1 / 2}, \mathrm{Fc}, \mathrm{Fc}^{+}=\left(\mathrm{E}_{\mathrm{ap}}+\mathrm{E}_{\mathrm{cp}}\right) / 2=(0.45+0.33) / 2=0.40 \mathrm{~V}\right]$ related to the Ag-wire reference electrode and then HOMO/LUMO energy levels of the polymers were calculated using the equation: $\mathrm{HOMO} / \mathrm{LUMO}=-\left(4.8-\mathrm{E}_{1 / 2}, \mathrm{Fc}, \mathrm{Fc}^{+}+\mathrm{E}_{\mathrm{ox} / \mathrm{red}}\right.$, onset $)$, where $\mathrm{E}_{\mathrm{ox} / \mathrm{red}}$ onset, is the onset oxidation or reduction potentials relative to the $\mathrm{Ag}$ wire reference electrode. ${ }^{233,236}$ The LUMO energy levels of polymers could be also calculated by subtracting the optical band gap from the respective HOMO energy levels $\left(\mathrm{LUMO}=\mathrm{HOMO}-\mathrm{Eg}_{\mathrm{g}}{ }^{\mathrm{opt}}\right)$.

Table 3.2 summarizes the electrochemical properties of both $\mathbf{P 5}$ and P6 polymers, which were measured from analysis of their respective cyclic voltammetry results (see Figure 3.7). The HOMO ${ }^{\text {ele }} / \mathrm{LUMO}^{\text {ele }}$ energy levels of $\mathbf{P 5}$ and $\mathbf{P 6}$ are $5.25 /-4.03 \mathrm{eV}$ and $-5.22 /-4.20 \mathrm{eV}$, respectively. The $\mathrm{LUMO}^{\text {ele }}$ for $\mathbf{P 6}$ is lower than that of $\mathbf{P 5}$, whereas their $\mathrm{HOMO}^{\text {ele }}$ levels are comparable. This could be associated 
with the donor moieties used where $\mathbf{P 6}$ contains a weak donor due to an electron withdrawing oxygen atom than thiophene of $\mathbf{P 5}$ in the alkyl side chain of the polymer backbone. Therefore, the bandgap (both $\mathrm{Eg}^{\mathrm{ele}}$ and $\left.\mathrm{Eg}^{\mathrm{opt}}\right)$ of $\mathbf{P 6}(1.02 \mathrm{eV})$ is lower than that of $\mathbf{P 5}(1.22 \mathrm{eV})$.

Table 3.2. Electrochemical properties of $\mathbf{P 5}$ and $\mathbf{P 6}$ polymers.

\begin{tabular}{|l|l|l|l|l|l|l|}
\hline $\begin{array}{l}\text { Poly } \\
\text { mer }\end{array}$ & $\begin{array}{l}\mathbf{E}_{\text {ox }}^{\text {onset }^{(\mathbf{V}}} \\
(\mathbf{V})\end{array}$ & $\begin{array}{l}\mathbf{E}_{\text {red }^{\text {onset }}} \\
(\mathbf{V})\end{array}$ & $\begin{array}{l}\mathbf{H O M O}^{\text {ele }} \\
(\mathbf{e V})\end{array}$ & $\begin{array}{l}\mathbf{L U M O}^{\text {ele }} \\
(\mathbf{e V})\end{array}$ & $\begin{array}{l}\mathbf{L U M O}^{\text {opt }} \\
(\mathbf{e V})\end{array}$ & $\begin{array}{l}\mathbf{E g}^{\text {ele }} \\
(\mathbf{e V})\end{array}$ \\
\hline P5 & 0.85 & -0.37 & $-5.25^{\mathrm{a}} /-$ & $-4.03^{\mathrm{a}} /$ & $-3.96^{\mathrm{b}}$ & $1.22^{\mathrm{c}} /$ \\
& & & $4.93^{\mathrm{d}}$ & $-3.07^{\mathrm{d}}$ & & $1.86^{\mathrm{d}}$ \\
\hline P6 & 0.82 & -0.20 & $-5.22^{\mathrm{a}} /$ & $-4.20^{\mathrm{a}} /$ & $-4.00^{\mathrm{b}}$ & $1.02^{\mathrm{c}} /$ \\
& & & $-4.99^{\mathrm{d}}$ & $-3.12^{\mathrm{d}}$ & & $1.88^{\mathrm{d}}$ \\
\hline
\end{tabular}

${ }^{\mathrm{a}} \mathrm{HOMO}^{\mathrm{ele}} / \mathrm{LUMO}^{\mathrm{ele}}=-\left(4.8-\mathrm{E} 1 / 2, \mathrm{Fc}, \mathrm{Fc}++\mathrm{E}_{\mathrm{ox} / \mathrm{red}}\right.$, onset $) .{ }^{\mathrm{b}} \mathrm{LUMO}^{\mathrm{opt}}=\mathrm{Eg}^{\mathrm{opt}}+$ $\mathrm{HOMO}^{\text {ele }} \cdot{ }^{\mathrm{c}} \mathrm{Eg}^{\mathrm{ele}}=\mathrm{LUMO}^{\text {ele }}-\mathrm{HOMO}^{\text {ele }} .{ }^{\mathrm{d}} \mathrm{DFT}$ calculated
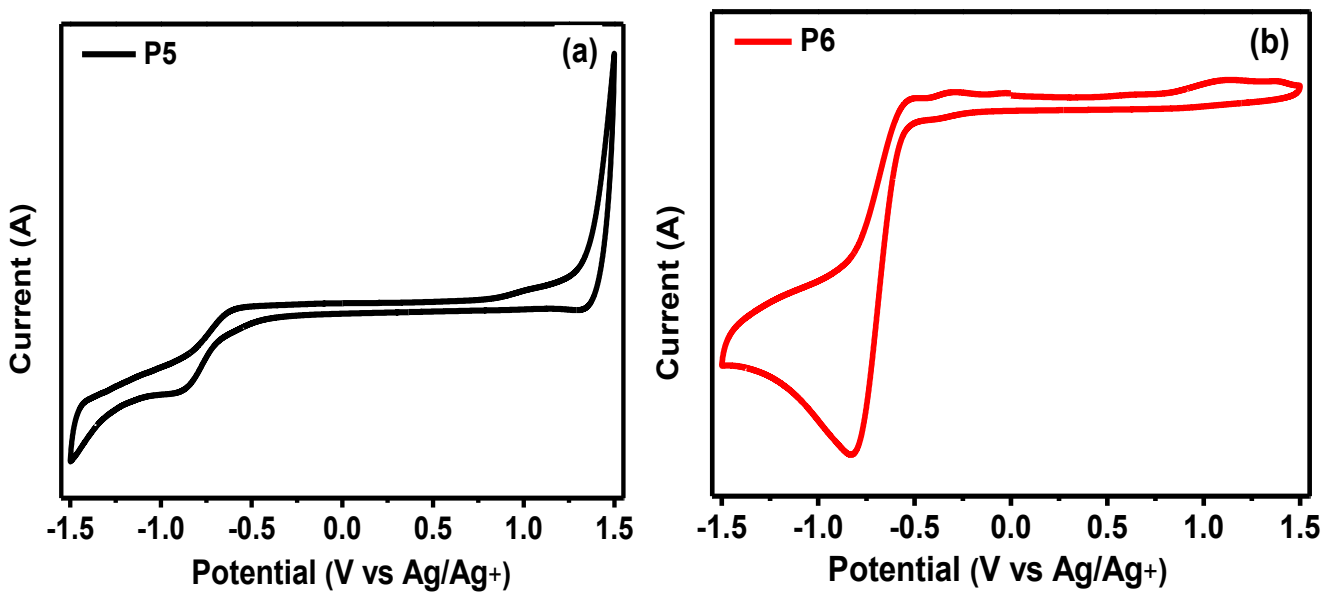


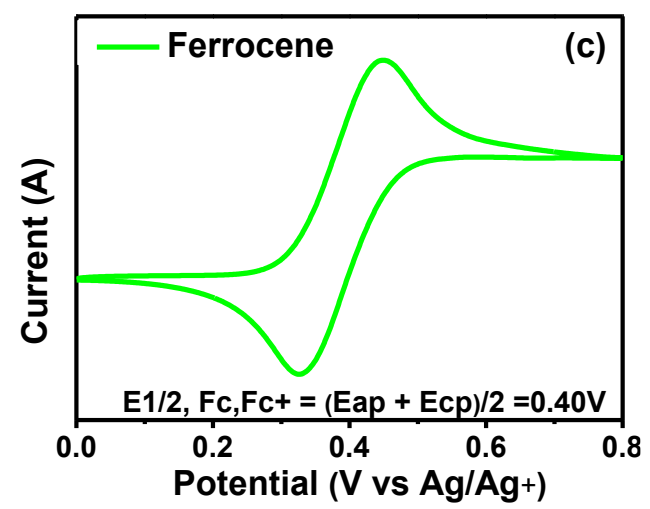

Figure 3.7. The cyclic voltammograms of $\mathbf{P 5}$ (a) and $\mathbf{P 6}$ (b) polymer films (c) Ferrocene measured in $0.1 \mathrm{~mol} / / \mathrm{lit} \mathrm{Bu}_{4} \mathrm{NPF}_{6}$ acetonitrile solutions at a scan rate of 50 $\mathrm{mVs}^{-1}$.

DFT calculation at the B3LYP/6-31G $(\mathrm{d}, \mathrm{p})$ level was performed to get more understanding on the electronic structure of both polymers. As shown in Figure 3.8, LUMO orbitals are mainly localized on the electron accepting moiety, $\gamma$-lactonePechmann dye, whereas HOMO orbitals are delocalized across the conjugated polymer backbone, which is an indication for the planarity of the polymer backbone, thus promoting the intra/intermolecular charge transfer and broadening the absorption edge. In comparison with $\mathbf{P 6}$, the thiophene side group in $\mathbf{P 5}$ is not in the same plane with its polymer backbone. The twisting with a torsion angle of $52^{\circ}$ could negatively affect its intermolecular interaction and electrochemical properties of $\mathbf{P 5}$. Similar results have also been reported in other BDT-based D-A copolymers. ${ }^{234,237,238}$ 


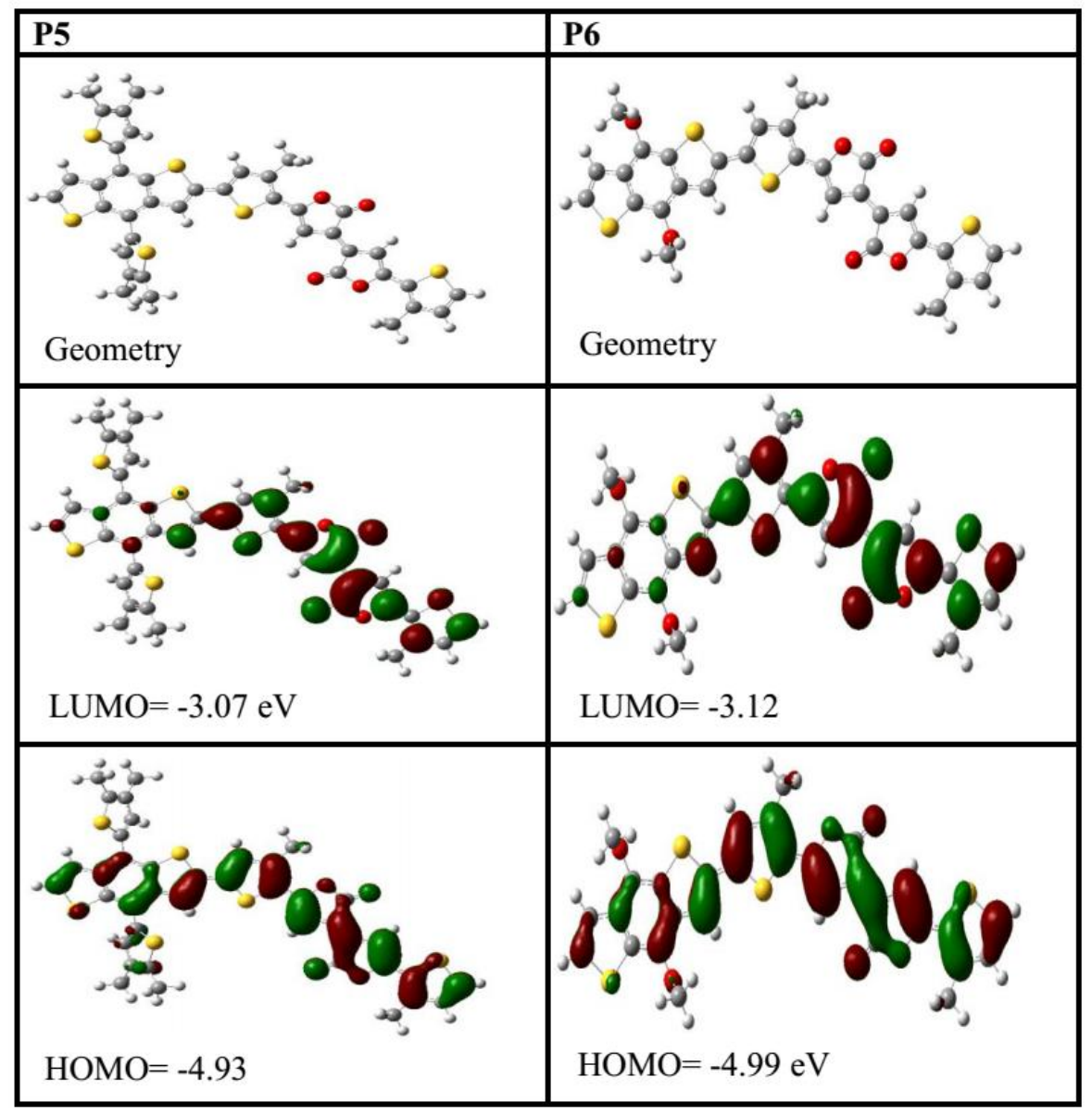

Figure 3.8. DFT calculation at B3LYP/6-31G(d,p) level for geometry, molecular orbital distribution, and HOMO/LUMO energy levels of P5 (left) and P6 (right). For computational simplicity, the alkyl chains are replaced with methyl groups.

\subsubsection{Field-Effect Transistor Devices}

The field-effect transistor properties of the polymers were evaluated using a bottom-gate/bottom-contact transistor configuration. A heavily n-doped silicon 
wafer $<100\rangle$ with a 300 -nm thermally grown silicon dioxide $\left(\mathrm{SiO}_{2}\right)$ was used as the substrate/gate electrode. The drain-source (D-S) gold contacts were fabricated by photolithography. Before usage, the wafers were sonicated using ethanol, acetone, and IPA for 10 minutes each and dehydrated on a hot plate for 10 minutes followed by UV-Ozone treatment for 15 minutes. Then, the surface was immersed in an octadecyltrichlorosilane (OTS-18) solution for over $12 \mathrm{~h}$ in order to create selfassembled monolayer (SAM). Finally, the samples were rinsed several times with toluene and dried at $110{ }^{\circ} \mathrm{C}$ for several hours. P5 and P6 solution were prepared in 1,2-dichlorobenzene with a concentration of $3 \mathrm{mg} / \mathrm{mL}$ The solution was stirred overnight at $70{ }^{\circ} \mathrm{C}$ were spin-coated on the substrate at $2000 \mathrm{rpm}$ for $60 \mathrm{~s}$. The annealing process was carried out over a hotplate for $30 \mathrm{~min}$ at $120{ }^{\circ} \mathrm{C}$ in the glovebox. Field-effect characteristics of the devices were recorded using a probe station (Anexus) and a source meter (Agilent, B2902A) in $\mathrm{N}_{2}$ filled glovebox.

Charge transport properties $\mathbf{P 5}$ and $\mathbf{P 6}$ in thin films were measured in the organic field-effect transistors (OFETs) using a bottom-gate/bottom-contact (BG/BC) configuration in $\mathrm{N}_{2}$ filled glovebox. The transfer and output characteristics of $\mathbf{P 5}$ and $\mathbf{P 6}$ are presented in Figure 3.9. One can see that P5 exhibits a p-type charge transport behavior with an average mobility of $1.98 \times 10^{-5} \mathrm{~cm}^{2} \mathrm{~V}^{-1} \mathrm{~s}^{-1}$, whereas $\mathbf{P 6}$ exhibits an ambipolar charge transport behavior with dominating hole mobility over the electron mobility (see Table 3.3). Specifically, the hole $\left(\mu_{\mathrm{h}}\right)$ and electron $\left(\mu_{\mathrm{e}}\right)$ mobilities of the as-prepared OFETs based on P6 polymer were measured to be $2.60 \times 10^{-5}$ and 1.23 
$\times 10^{-6} \mathrm{~cm}^{2} \mathrm{~V}^{-1} \mathrm{~s}^{-1}$, respectively. When the films were subjected to thermal annealing at $120{ }^{\circ} \mathrm{C}$, an increase of the carrier mobility was observed for both polymers. While the devices involving P5 as the semiconducting layer showed an increased hole mobility to $1.10 \times 10^{-4} \mathrm{~cm}^{2} \mathrm{~V}^{-1} \mathrm{~s}^{-1}$, the hole- and electron mobility of the P6 based OFETs increased to $1.50 \times 10^{-4}$ and $3.05 \times 10^{-6} \mathrm{~cm}^{2} \mathrm{~V}^{-1} \mathrm{~s}^{-1}$, respectively.

The relative low mobilities of the polymers might be due to the lack of their stability in air, as the HOMO level of these polymers is high (low ionization potential) and thus they could be easily oxidized by air. This has been observed in bottom-gate/top-contact (BG/TC) OFET devices, which have been fabricated and tested under the ambient condition to evaluate the stability of the polymer. Both polymers were revealed to be air-sensitive with a quick deterioration of the OFET performance. Therefore, we expect the polymers to perform better in a FET device with top-gate configurations or improved interfacial passivation of charge carrier traps. 53,230 
Table 3.3. Performance characteristics of OFET devices with $\mathbf{P 5}$ and $\mathbf{P 6}$ polymer semiconductor on an OTS-18-treated substrate fabricated and measured under a nitrogen atmosphere.

\begin{tabular}{|c|c|c|c|c|c|c|c|}
\hline \multirow[b]{2}{*}{$\begin{array}{l}\text { Poly } \\
\text { mer }\end{array}$} & \multirow[b]{2}{*}{$\begin{array}{l}\text { Annealing } \\
\text { Temp }\left({ }^{\circ} \mathrm{C}\right)\end{array}$} & \multicolumn{3}{|c|}{ p-type } & \multicolumn{3}{|c|}{ n-type } \\
\hline & & $\begin{array}{c}\mu_{\mathrm{h}} \\
\left(\mathrm{cm}^{2} \mathbf{V}^{-1} \mathbf{s}^{-1}\right)\end{array}$ & Ion/off & $\begin{array}{l}V_{\text {th }} \\
\text { (V) }\end{array}$ & $\begin{array}{c}\mu_{\mathrm{e}} \\
\left(\mathbf{c m}^{2} \mathbf{V}^{-1} \mathbf{s}^{-1}\right)\end{array}$ & Ion/off & $\begin{array}{l}V_{\text {th }} \\
\text { (V) }\end{array}$ \\
\hline \multirow[t]{2}{*}{ P5 } & r.t & $1.98 \times 10^{-5}$ & $\sim 10^{3}$ & -15 & - & - & - \\
\hline & 120 & $1.10 \times 10^{-4}$ & $\sim 10^{3}$ & -12 & - & - & - \\
\hline \multirow[t]{2}{*}{ P6 } & r.t & $2.60 \times 10^{-5}$ & $\sim 10^{3}$ & -7.5 & $1.23 \times 10^{-6}$ & $\sim 10$ & 27 \\
\hline & 120 & $1.50 \times 10^{-4}$ & $\sim 10^{3}$ & -12 & $3.05 \times 10^{-6}$ & $\sim 10$ & 30 \\
\hline
\end{tabular}
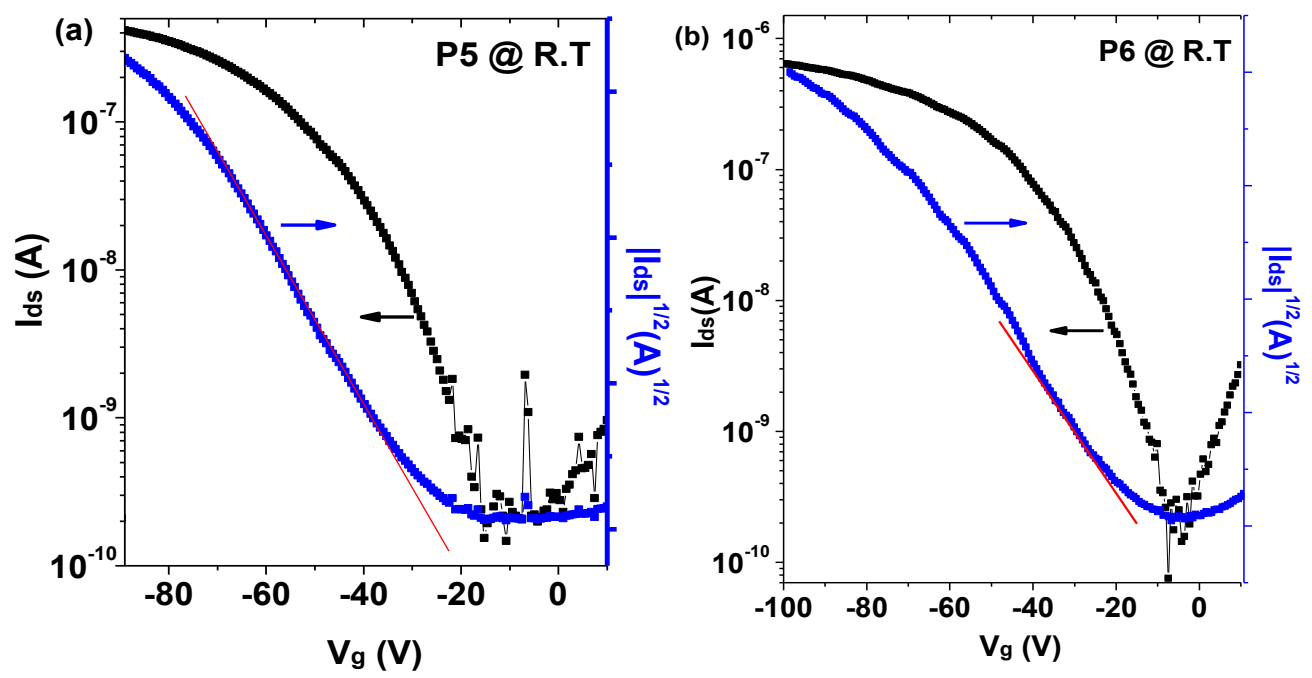

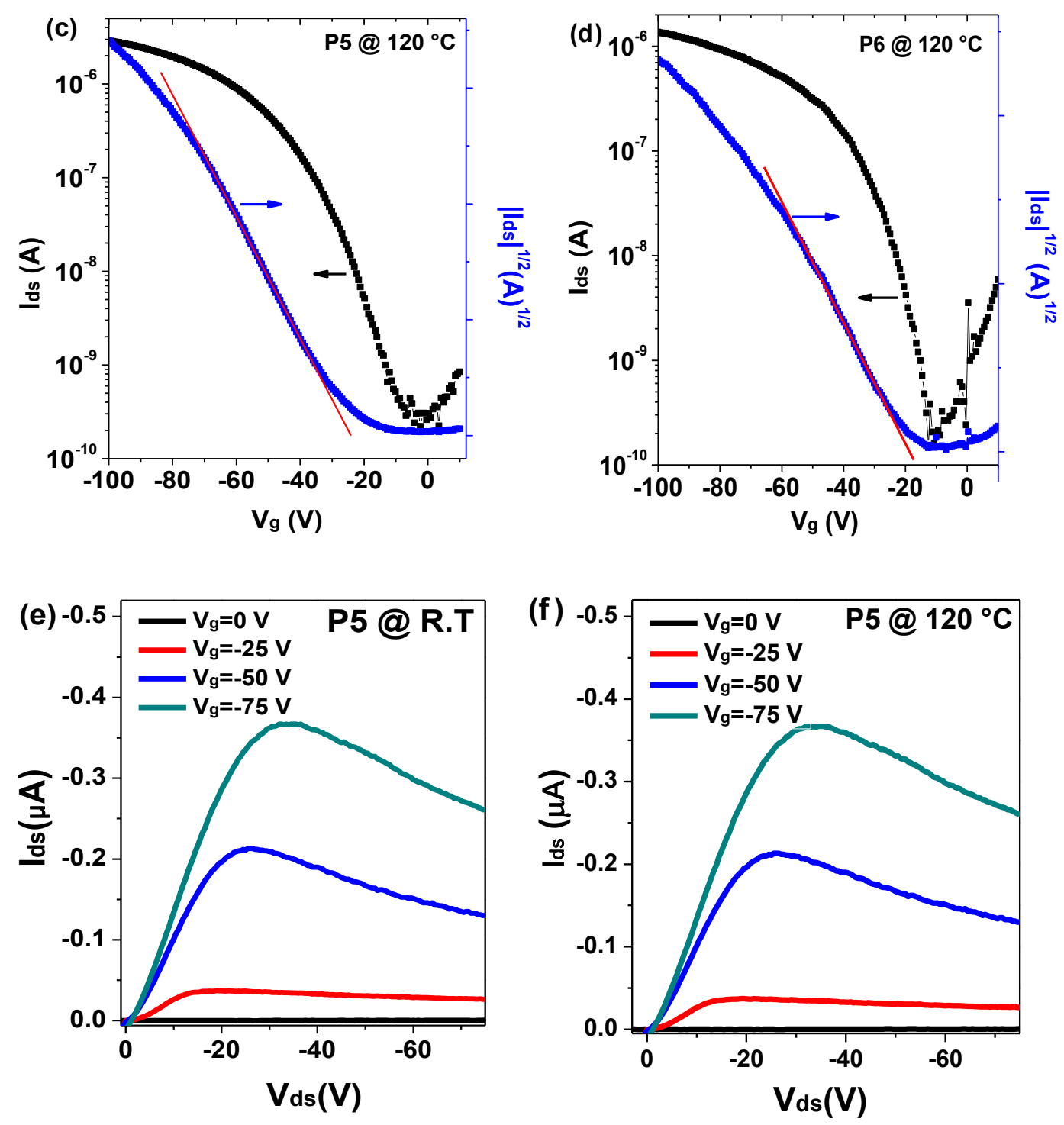

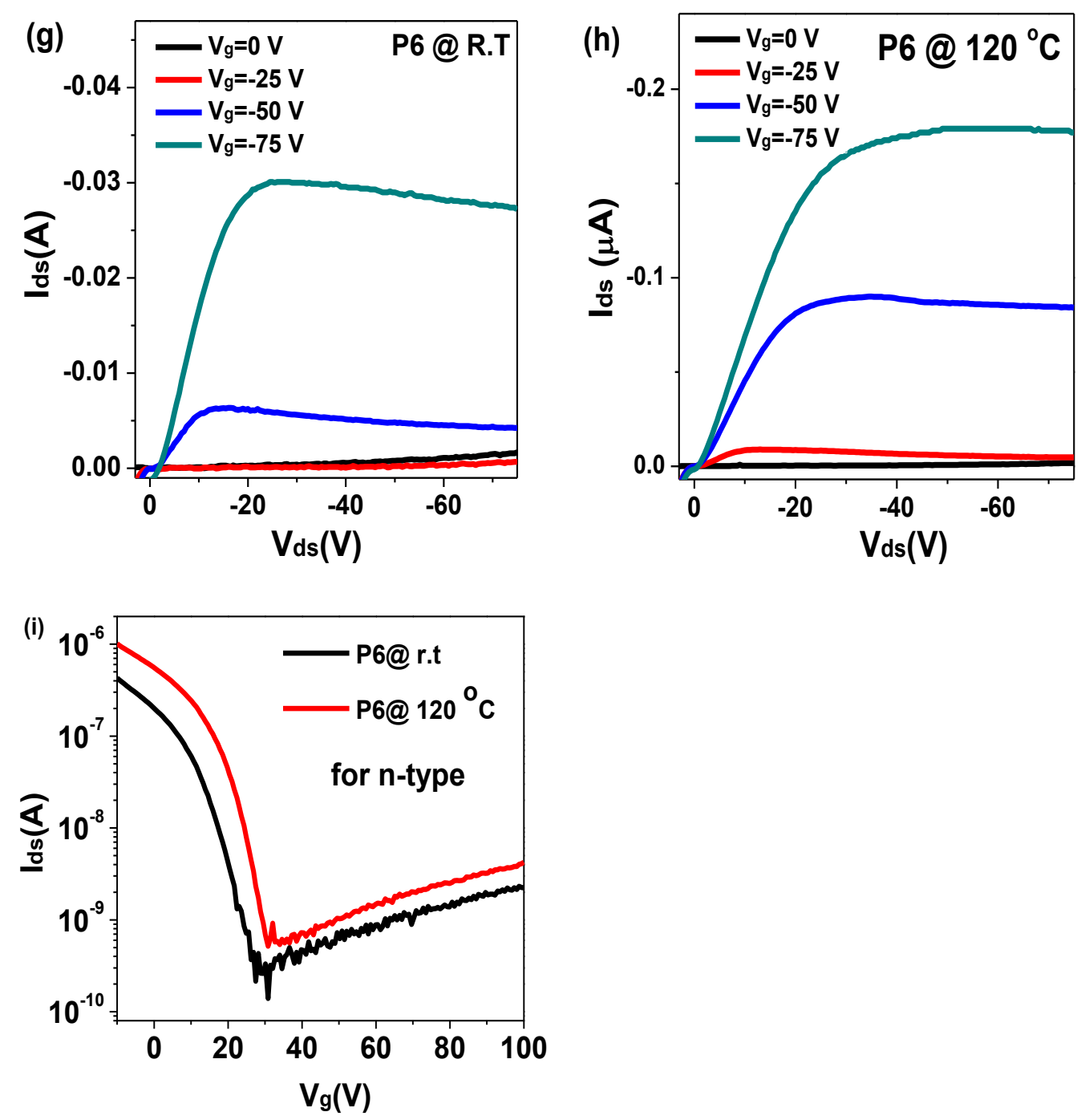

Figure 3.9. Typical transfer curves (a to d) and output characteristics curves (e to h) of $\mathbf{P 5}$ and $\mathbf{P 6}$ based OFET devices measured under the $\mathrm{N}_{2}$-filled glovebox. OFETs devices were prepared on OTS-18-modified $\mathrm{Si} / \mathrm{SiO}_{2}$ substrate $(\mathrm{W} / \mathrm{L}=10 \mathrm{~mm} / 5 \mu \mathrm{m}$ devices). 


\subsection{Conclusion}

In summary, two conjugated D-A polymers based on $\gamma$-lactone-Pechmann dye as the electron acceptor moiety, which is alternating with 2D-benzo[1,2-b:4,5$b^{\prime}$ ]dithiophene (2D-BDT) and alkoxy-benzo[1,2-b:4,5-b']dithiophene (1D-BDT) electron donor moiety, were synthesized and characterized. Based on DFT calculation $\gamma$-lactone-Pechmann dye acceptor moiety exhibited superior properties such as higher degree of coplanarity and stronger electron-accepting property than its lactam analogs. UV-vis-NIR absorption and cyclic voltammetry results reveal a broad absorption extended to $1000 \mathrm{~nm}$ and narrow band gap (1.22 eV for P5 and 1.02 eV for P6) with deep LUMO energy level. The thin film field-effect transistors of P5 and $\mathbf{P 6}$ showed p-type and ambipolar semiconducting properties, respectively. These results show that $\gamma$-lactone-Pechmann dye could be used as the potential electronaccepting building block for the construction of new conjugated D-A polymers.

\section{The content in Chapter 3 has been published in}

- Dyes and Pigment DIO: 10.1016/j.dyepig.2016.07.013 


\section{CHAPTER 4}

Synthesis and Characterization of tertbutoxycarbonyl (t-BoC)-Isoindigo based DonorAcceptor Conjugated Polymer and the Effect of tBoC Deprotection Method on the Optoelectronics properties

\subsection{Introduction}

Conjugated polymer semiconductors combine the electrical conductivities of metals with the mechanical properties of plastics like flexibility and solution processability. Thus, which could enable cheap and flexible organic electronics devices such as organic field-effect transistors (OFETs) ${ }^{239-244}$, organic photovoltaics $(\mathrm{OPVs})^{237,245-249}$, and Organic light emitting diodes (OLEDs) ${ }^{250,251}$ Introducing alkyl chains to the backbone of conjugated polymers is the most applicable strategy to modulate their solubility and then enable solution processability for film deposition of conjugated polymers via spin coating, inkjet printing, or blade coating. However, unlike to small molecules, the bulky alkyl side chains in the conjugated polymers 
could prevent them from compact packing through self-aggregation or the formation ordered structures and a widespread $\pi-\pi$ stacking. Thus, it could decrease absorption band, hinder charge carrier transport, and stability. Therefore, a proper rational design of solubilizing alkyl side group (its type, position, and length) in conjugated polymer backbone is quite important to balance the trade-off between the structures of conjugated polymers and their solution processability.

The possibility of removing solubilizing side groups after the polymerization reaction and deposition of polymer films on the flexible/transparent substrate gives another paradigm in designing conjugated polymers for organic electronics applications. ${ }^{252,253}$ For instance, side groups like silyl, ${ }^{254,255}$ and ester, ${ }^{256-258}$ substituents have been developed and enabled the deposition of thin films from their solution followed by their post-processing elimination. Among these latent groups, an ester type of side group, tert-butoxycarbonyl (t-BoC) has recently used as an effective latent group in conjugated polymers that can be easily removed after thermal treatment of their thin film..$^{259-263}$

Donor-Acceptor type of $\pi$-conjugated polymers based on tert-butoxycarbonyl (t-BoC) substituted isoindigo as an acceptor moiety and the elimination of bulky tBoC side group resulted in the emergence of hydrogen bonding interactions between $\mathrm{N}-\mathrm{H} \cdots \mathrm{O}=\mathrm{C}$ functional groups. It may result in increased charge carrier mobilities of the resulting copolymers after the removal of $\mathrm{t}-\mathrm{BoC}$ by chemical or thermal treatment method in thin film organic electronics devices (such as OFETs and OPVs). Which 
may arise from an enhanced planarity and intermolecular ordering of the isoindigo type of building blocks. For instance, Liu et al. has reported a series of D-A conjugated polymers based on thermally cleavable t-BoC side group attached to the lactam electron-withdrawing group containing acceptor moieties such as indigo, isoindigo, diketopyrrolopyrrole (DPP), and quinacridone have demonstrated the formation of intermolecular hydrogen bonding in the polymer films after the removal of t-BoC side group via thermal treatment. ${ }^{259-261}$

This Chapter presents the design, synthesis, and characterization of D-A conjugated polymers based on t-BoC-isoindigo as an acceptor unit. Where t-BoC is used to protect the reactive $\mathrm{N}-\mathrm{H}$ in isoindigo during the polymerization reaction and helps to enhance the solubility of the monomer, and its polymer during the polymerization reaction and also solution processability for thin film organic electronics device fabrication. In addition to this, the impact of t-BoC removal method (chemical vs. thermal) on the formation of an improved planar conjugated backbone of the polymer and thus the formation of $\mathrm{N}-\mathrm{H} \cdots \mathrm{O}=\mathrm{C}$ hydrogen bonding network is also investigated and presented using TGA, UV-vis-NIR, CV and OFET experiments (see Figure 4.1). ${ }^{264-269}$ 
<smiles>CNC(=O)OC(C)(C)C</smiles>


Hydrogen bond network

Figure 4.1. Illustration for methods of t-BoC removal and the possible hydrogen bond network.

\subsection{Experimental}

Materials. Unless stated otherwise, starting materials and anhydrous solvents were obtained from Sigma-Aldrich and used without further purification. 6,6'dibromoisoindigo, ${ }^{\mathbf{2 7 0}}$ t-BoC-isoindigo (t-BoC-ID) ${ }^{\mathbf{2 7 1}}$ was prepared according to reported procedures. Donor moieties like (4,8-bis(4,5-didecylthiophen-2yl)benzo[1,2-b:4,5-b']dithiophene-2,6-diyl)bis(trimethylstannane) (2D-BDT) was obtained commercially from SunaTech Inc. and used without further purification. All the reactions were carried out under an inert atmosphere.

Electrochemical Characterization. Electrochemical measurements were conducted on a CHI006D electrochemical analyzer with a three-electrode system 
under a nitrogen atmosphere in a deoxygenated anhydrous acetonitrile solution of tetra-n-butylammonium hexafluorophosphate $(0.1 \mathrm{M})$. A glassy carbon electrode was used as a working electrode, a platinum wire was used as the counter electrode, and an Ag wire was used as the pseudo-reference electrode. The sample films for electrochemical measurements were coated on the surface of the glassy carbon electrode. The $\mathrm{CV}$ curves were calibrated using the ferrocene/ferrocenium $\left(\mathrm{Fc} / \mathrm{Fc}^{+}\right)$ redox couple as an external standard which was measured under the same condition before and after the measurement of samples. The energy level of $\mathrm{Fc} / \mathrm{Fc}+$ was assumed at $-4.8 \mathrm{eV}$ to vacuum. ${ }^{272}$ The half-wave potential of the ferrocene/ferrocenium $\left(\mathrm{Fc} / \mathrm{Fc}^{+}\right)$redox couple $\left(\mathrm{E}_{1 / 2}, \mathrm{Fc}, \mathrm{Fc}^{+}\right)$was estimated from $\mathrm{E}_{1 / 2}$, $\mathrm{Fc}, \mathrm{Fc}^{+}=\left(\mathrm{E}_{\mathrm{ap}}+\mathrm{E}_{\mathrm{cp}}\right) / 2$, where $\mathrm{E}_{\mathrm{ap}}$ and $\mathrm{E}_{\mathrm{cp}}$ are the anodic and cathodic peak potentials, respectively. As indicated on Figure 4.8, the half-wave potential of $\mathrm{Fc} / \mathrm{Fc}^{+}$is to be $0.463 \mathrm{~V}\left[\mathrm{E}_{1 / 2}, \mathrm{Fc}, \mathrm{Fc}^{+}=\left(\mathrm{E}^{\mathrm{ap}}+\mathrm{E}^{\mathrm{cp}}\right) / 2=(0.497+0.43) / 2=0.463 \mathrm{~V}\right]$ related to the $\mathrm{Ag} / \mathrm{Ag}^{+}$reference electrode. The LUMO energy levels of monomers and polymers were calculated using the equation: $\mathrm{E}_{\mathrm{LUMO}}=-\left(4.8-\mathrm{E}_{1 / 2}, \mathrm{Fc}, \mathrm{Fc}^{+}+\mathrm{E}_{\text {red }}\right.$, onset $)=-$ $\left(4.33+\mathrm{E}_{\text {red, }}\right.$ onset $) \mathrm{eV}$, where $\mathrm{E}_{\text {red }}$, onset is the onset reduction potential relative to the $\mathrm{Ag} / \mathrm{Ag}^{+}$reference electrode. ${ }^{233,236}$ The HOMO energy levels of monomers were calculated by subtracting the optical band gap value from the respective LUMO energy levels $\left(\mathrm{E}_{\text {HOMO }}=\mathrm{E}_{\mathrm{LUMO}}-\mathrm{E}_{\mathrm{g}}\right.$, opt $)$. The HOMO energy levels of polymers were estimated by equation: $\mathrm{E}_{\text {номо }}=-\left(4.33-\mathrm{E}_{1 / 2}, \mathrm{Fc}, \mathrm{Fc}^{+}+\mathrm{E}_{\mathrm{ox}}\right.$, onset $)=-(4.33+$ 
$E_{o x}$, onset) $e V$, where $E_{o x}$, onset is the onset oxidation potential relative to the $\mathrm{Ag} / \mathrm{Ag}_{+}$ reference electrode..$^{233,236}$

Field-Effect Transistor Fabrication and Characterization. Bottomgate/top-contact OFETs were fabricated using heavily doped Si wafer as the bottom gate electrode with $300 \mathrm{~nm}$ of the $\mathrm{SiO}_{2}$ layer as the gate dielectric. Gold source and drain electrodes $(60 \mathrm{~nm})$ were patterned by the vacuum thermal evaporation. The substrate was cleaned in acetone, isopropanol, and dried on the hot plate at $110^{\circ} \mathrm{C}$ then it was further cleaned with piranha solution $\left(\mathrm{H}_{2} \mathrm{SO}_{4}: \mathrm{H}_{2} \mathrm{O}_{2}, 3: 1 \mathrm{v} / \mathrm{v}\right)$, wash with deionized water and dried at $100{ }^{\circ} \mathrm{C}$. After that, the substrate was immersed in the octadecyltrichlorosilane (ODTS) solution in toluene solvent for overnight, rinsed with toluene to remove excess ODTS, and dried on the hot plate at $110{ }^{\circ} \mathrm{C}$. The polymers dissolved in 1,2-dichlorobenzene at a concentration of $10 \mathrm{mg} \mathrm{mL}^{-1}$ by stirring for 1 day at $60{ }^{\circ} \mathrm{C}$. The polymer films were spin-cast at $1500 \mathrm{rpm}$ and annealed at each temperature of interest for $15 \mathrm{~min}$. Finally, the gold electrode (60 $\mathrm{nm}$ ) was thermally evaporated in vacuum through a shadow mask with two different dimension length (L) of $200 \mu \mathrm{m}$ and channel width (W) of $4.0 \mathrm{~mm}$. The field effect mobilities were extracted from the saturation regime using the equation: $\mu$ sat $=(2$ $I \mathrm{ds} L) /\left(W C \mathrm{i}(V g-V \text { th })^{2}\right)$, where $I d s$ denotes the saturation drain current, $C \mathrm{i}$ is the capacitance $\left(\mathrm{Ci}=10.8 \times 10^{-9} \mathrm{~F} \mathrm{~cm}^{-2}\right)$ of $\mathrm{SiO}_{2}$ dielectric, $V d$ is the gate bias, and $V$ th is the threshold voltage. All OFET devices fabrication and measurement were done in the air without any protective atmosphere. 


\subsubsection{Synthesis of monomers}

\section{Synthesis of 6,6'-Dibromoisoindigo $(20)^{270}$}

6-Bromooxindole (2.00 g, $9.44 \mathrm{mmol})$ and 6-bromoisatin $(2.13 \mathrm{~g}, 9.44 \mathrm{mmol})$ were dissolved in acetic acid $(100 \mathrm{~mL})$, followed by the addition of concentrated $\mathrm{HCl}$ solution $(0.4 \mathrm{~mL})$, with the reaction mixture vigorously stirring under reflux for $24 \mathrm{~h}$. After cooling to room temperature, the mixture was filtered. The solid was washed with water, ethanol and diethyl ether. After drying under a vacuum, the brown powder was obtained, Compound 20 (3.41 g, 100\%).

${ }^{1} \mathrm{H}$ NMR $\left(\mathrm{CDCl}_{3}, 300 \mathrm{MHz}\right) \delta(\mathrm{ppm}):$ 7.81(s, 2H), 7.68(d, 2H), $7.40(\mathrm{~s}, 2 \mathrm{H})$, $7.16(\mathrm{~s}, 2 \mathrm{H})$

(E)-di-tert-butyl 6,6'-dibromo-2,2' -dioxo-[3,3'-biindolinylidene]-1,1'-dicarbo xylate (t-BoC-ID) $(21)^{271}$

To a stirred solution of 6,6'-dibromoisoindigo (Compound 20) (1.5 g, 3.57 mmol) in dry tetrahydrofuran $(30 \mathrm{~mL})$ was added 4-Dimethylaminopyridine (DMAP) (43.65 mg, $0.36 \mathrm{mmol})$, triethylamine $(3.6 \mathrm{~g}, 35.7 \mathrm{mmol})$. The resulting solution was cooled to $0{ }^{\circ} \mathrm{C}$ and di-tert-butyl dicarbonate $\left(\mathrm{BoC}_{2} \mathrm{O}\right)(1.95 \mathrm{~g}, 8.93 \mathrm{mmol}$ was dissolved in a separate vial and added dropwise) the resulting reaction mixture was warmed to room temperature and was stirred for $24 \mathrm{~h}$. The reaction mixture was diluted with diethyl ether $(30 \mathrm{~mL})$ and was filtered through a plug of silica gel, washing with additional diethyl ether. The crude reaction mixture was concentrated, 
and recrystallized from diethyl ether: hexane (1:1) to afford bright orange needles $(1.87 \mathrm{~g}, 84 \%)$.

1H-NMR (300 MHz, $\left.\mathrm{CDCl}_{3}\right): \delta 8.95$ (dd, 1H), 7.81 (dd, 1H), 7.43 (ddd, 7.3, 1H), 7.17 (ddd, 7.4, 1.0 Hz, 1H), 1.68 (s, 9H).

\subsubsection{Synthesis of Polymers (P7 and P8a)}

\section{Synthesis of P7 polymer}

To a $25 \mathrm{~mL}$ round-bottom flask t-BoC protected dibromoisoindigo, t-BoC-ID (61.83 mg, $0.1 \mathrm{mmol}), \quad$ (4,8-bis(4,5-didecylthiophen-2-yl)benzo[1,2-b:4,5b']dithiophene-2,6-diyl)bis (trimethyl stannane), 2D-BDT (124.12 mg, $0.1 \mathrm{mmol}$ ), and $\left.\mathrm{Pd}\left(\mathrm{PPh}_{3}\right)_{4}\right)(2 \mathrm{~mol} \%, 2.3 \mathrm{mg})$ catalyst were added under nitrogen atmosphere. After three times degassing and purging with nitrogen, $10 \mathrm{~mL}$ of anhydrous toluene was added. The flask was charged with nitrogen through a freeze-pump-thaw cycle for three times. The mixture was stirred for $48 \mathrm{hr}$ at $85^{\circ} \mathrm{C}$ under nitrogen. After being cooled to room temperature, the reaction mixture was poured into $100 \mathrm{~mL}$ of methanol and filtered. The resulted crude product was then subjected to Soxhlet extraction with methanol, acetone, hexane, and chloroform in sequence. The chloroform fraction was evaporated to dryness and reprecipitated from methanol to give P7 as a black powder $(80 \mathrm{mg}, 58.3 \%) . \quad M_{\mathrm{n}}=17.7 \mathrm{kDa}, M_{\mathrm{w}}=26.1 \mathrm{kDa}, \mathrm{PDI}=$ 1.47 
Chemical method for Deprotection t-BoC group from P7 polymer to give (P8a)

To a solution of $\mathbf{P 7}(40 \mathrm{mg}, 0.029 \mathrm{mmol})$ in $\mathrm{CH}_{2} \mathrm{Cl}_{2}(10.0 \mathrm{~mL})$ at $0{ }^{\circ} \mathrm{C}$ was added $\mathrm{CF}_{3} \mathrm{COOH}(13 \mathrm{mg}, 0.116 \mathrm{mmol})$. The reaction mixture was allowed to warm to r.t and was stirred for $24 \mathrm{~h}$. The solvent was removed by rotavap to afford the desired product, P8a as a black solid (33 mg, 96.5\%). $M_{\mathrm{n}}=31.7 \mathrm{kDa}, M_{\mathrm{w}}=57.2 \mathrm{kDa}$, $\mathrm{PDI}=1.8$

\subsubsection{GPC traces for polymers and NMR spectra}

\subsubsection{GPC traces for polymers}
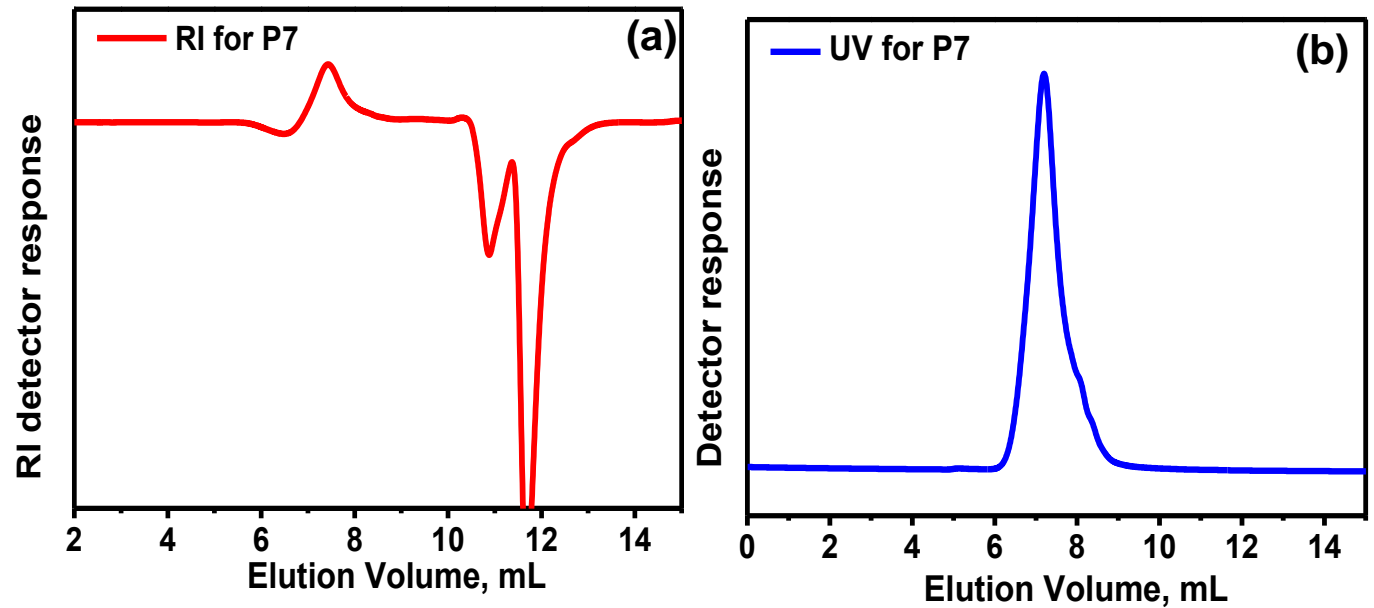

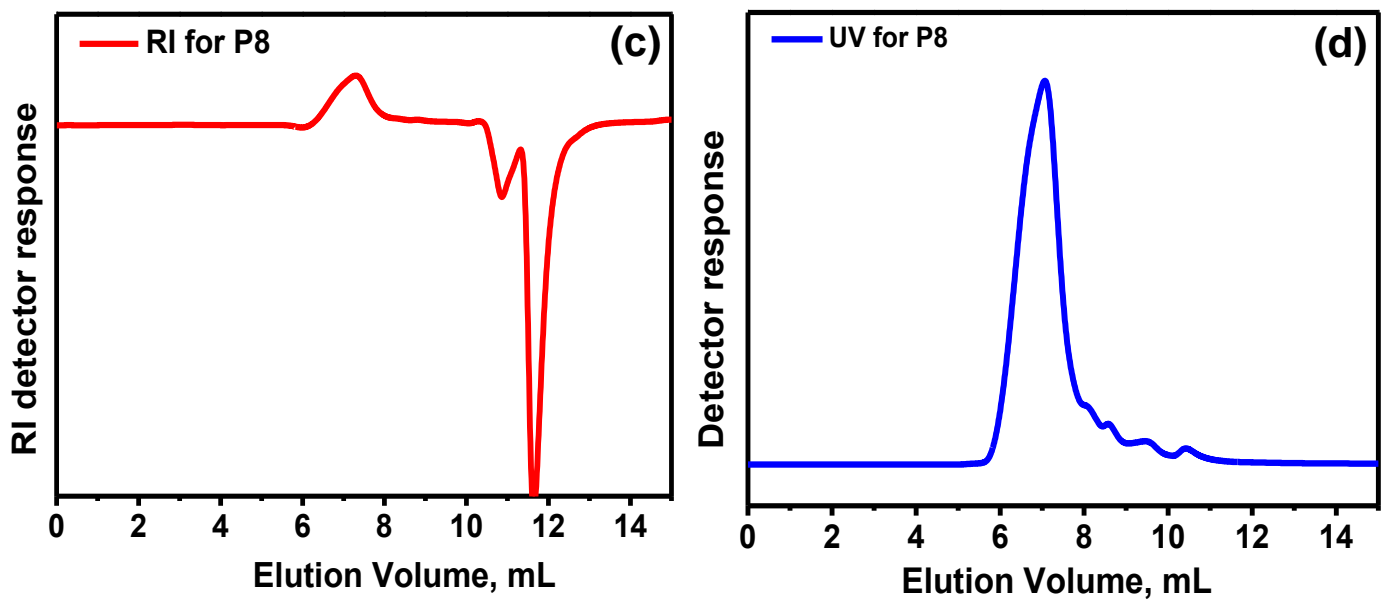

Figure 4.2. GPC traces for $\mathbf{P 7}$ and $\mathbf{P 8 a}$ (using $\mathrm{CHCl}_{3}$ as eluent at $80{ }^{\circ} \mathrm{C}$ ) from $\mathrm{RI}(\mathrm{a}$ and c) and UV (550 nm) (b and d) detectors, respectively.

\subsubsection{NMR spectra of monomers and polymers}

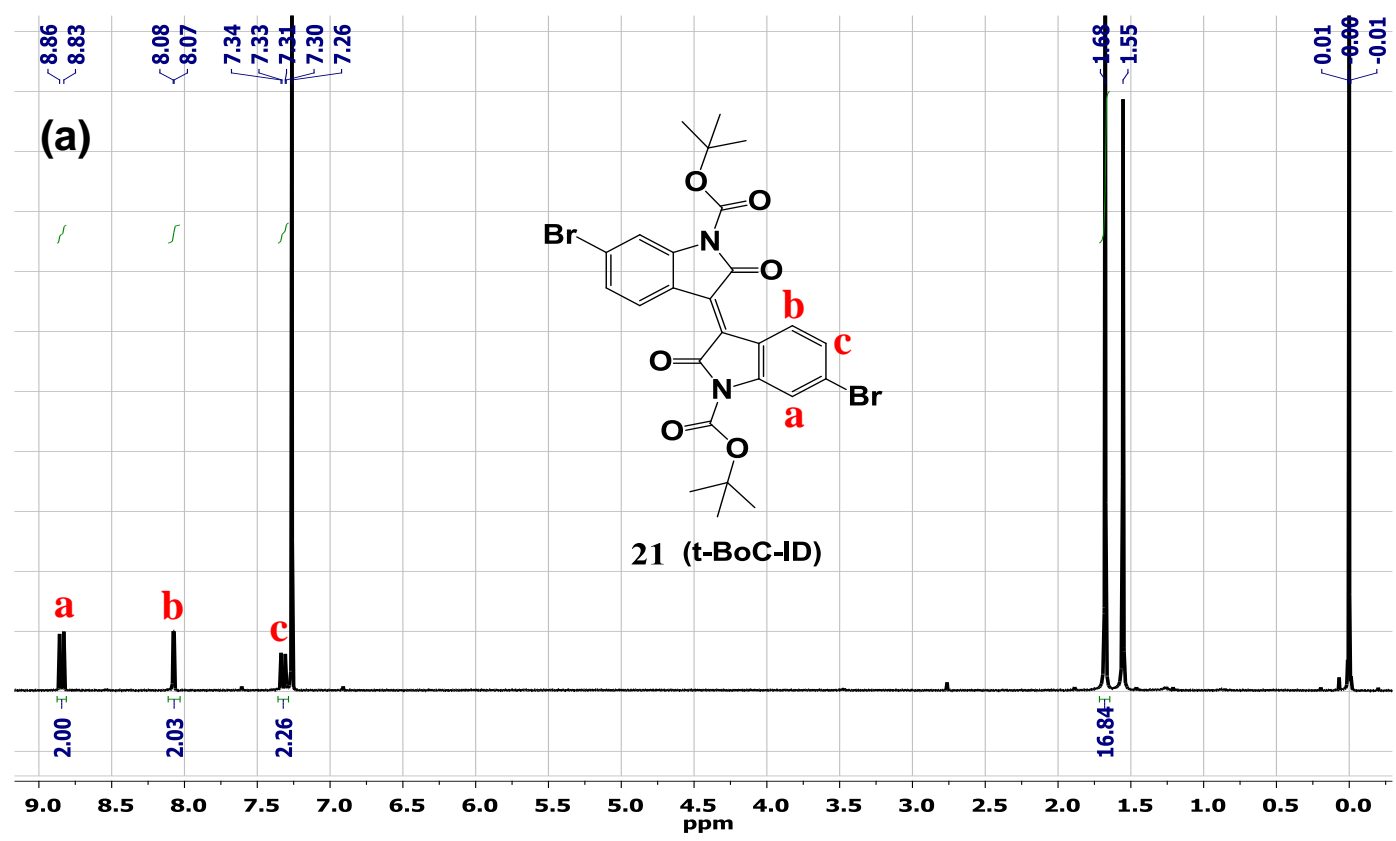

Figure 4.3. ${ }^{1} \mathrm{H}$ NMR (300 MHz) spectra for compound 21 (a) in $\mathrm{CDCl}_{3}$ solvent at R.T. 


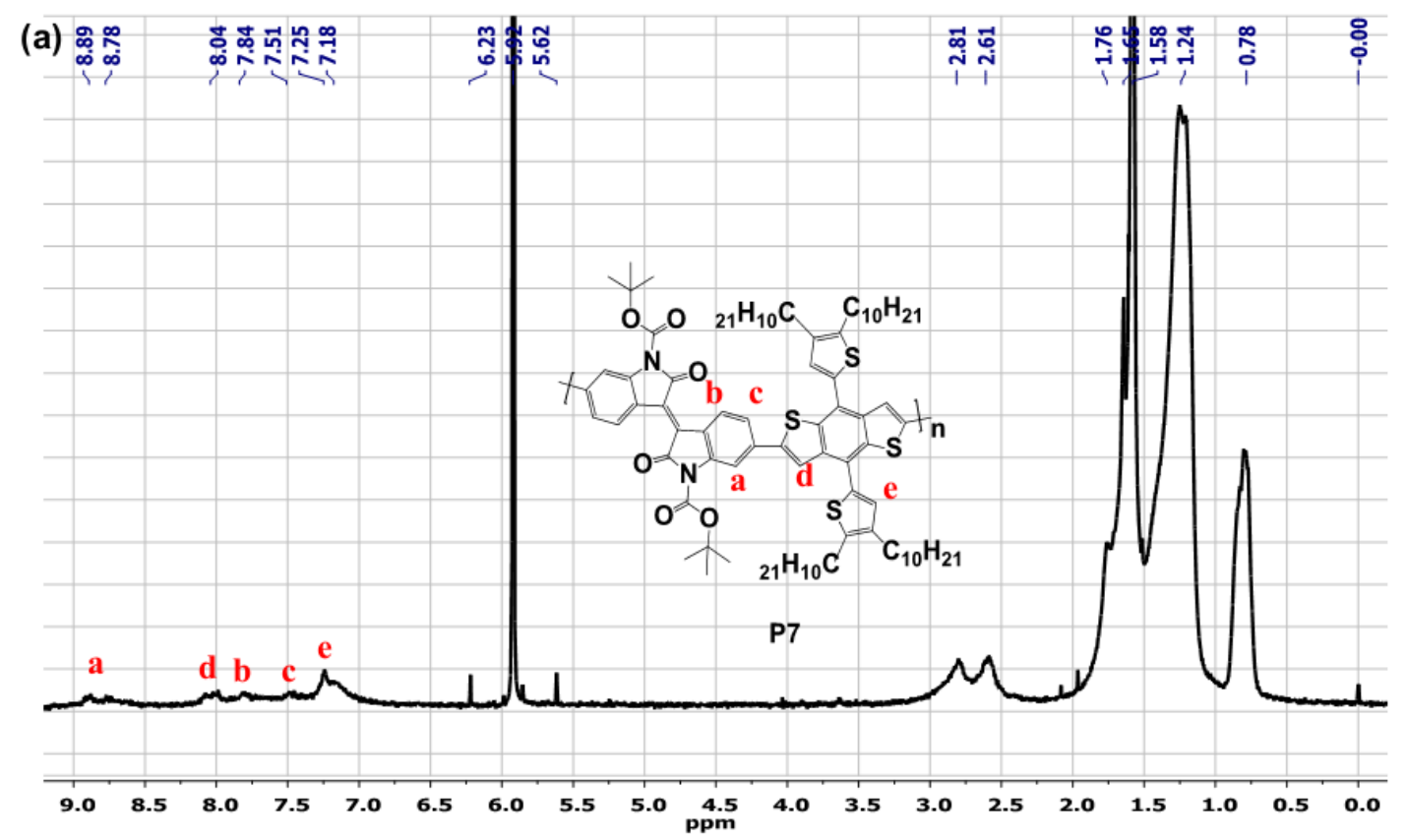

Figure 4.4. ${ }^{1} \mathrm{H}$ NMR (300 MHz) spectra for $\mathbf{P 7}$ (a) in $\mathrm{C}_{2} \mathrm{D}_{2} \mathrm{Cl}_{4}$ solvent at $100{ }^{\circ} \mathrm{C}$.

\subsection{Results and Discussion}

\subsubsection{Synthesis of Monomers and Polymers (P7 \& P8)}

As depicted in Scheme 8, t-BoC protected dibromoisoindigo (Compound 2) was synthesized according to the reported procedure. ${ }^{271}$ Then followed by Stille polycondensation with a donor moiety 4,8-bis(4,5-didecylthiophen-2-yl)benzo[1,2b:4,5-b']dithiophene-2,6-diyl)bis(trimethyl stannane) (2D-BDT) in the presence of $\mathrm{Pd}\left(\mathrm{PPh}_{3}\right)_{4}$ catalyst, dry toluene solvent, and refluxing at $85{ }^{\circ} \mathrm{C}$ for $48 \mathrm{~h}$ to afford a crude polymer P7. The polymer was purified via precipitation from methanol and subsequent Soxhlet extraction using methanol, acetone, hexane, and chloroform in 
sequence. The polymer $\mathbf{P 7}$ was collected from the chloroform fraction in a 58.3\% yield. The deprotection (removal of the t-BoC group) of the $\mathbf{P 7}$ polymer was achieved either by chemical or thermal treatment methods. In the chemical method, P7 was stirred in dichloromethane solvent containing $\mathrm{CF}_{3} \mathrm{COOH}$ at room temperature for $24 \mathrm{~h}$ to give P8a polymer in $96.5 \%$ yield as a black solid powder. At this point the solubility of the polymer P8a was found very poor in all chlorinated solvents, partly it could be due to the complete removal of a t-BoC protecting group of P7 and the formation of strong hydrogen bond network between the neighboring $\mathrm{N}-\mathrm{H} \cdots \mathrm{O}=\mathrm{C}$ functional groups of the resulting polymer, P8a. The removal of t-BoC side group from $\mathbf{P 7}$ is confirmed by collecting their respective FT-IR spectra (see Figure 4.5), where the $\mathrm{C}=\mathrm{O}$ stretching around $\sim 1780 \mathrm{~cm}^{-1}$ is disappeared in P8a after treatment with $\mathrm{CF}_{3} \mathrm{COOH}$. The $\mathrm{C}=\mathrm{O}$ from the lactam group in isoindigo is also shifted from $\sim 1730 \mathrm{~cm}^{-1}$ to lower wavenumber $1700 \mathrm{~cm}^{-1}$, which is an indication of the presence of hydrogen bonding network $\mathrm{N}-\mathrm{H} \cdots \mathrm{O}=\mathrm{C}$ after $\mathrm{t}-\mathrm{BoC}$ elimination. ${ }^{260,273}$ On the other hand, the deprotection $\mathbf{P 7}$ polymer was also achieved through annealing of its thin film at $200{ }^{\circ} \mathrm{C}$ under vacuum. The resulting polymer is labeled as $\mathbf{P 8 b}$ and then the molecular weights of the P7 and P8a polymers were evaluated by gel permeation chromatography (GPC) at $80{ }^{\circ} \mathrm{C}$ with $\mathrm{CHCl}_{3}$ as an eluent (see section 4.4.3). The number-average molecular weight $\left(\mathrm{M}_{\mathrm{n}}\right)$ /polydispersity index (PDI) of $\mathbf{P 7}$ and P8a is $17.7 \mathrm{kDa} / 1.4$ and $31.7 \mathrm{kDa} / 1.8$, respectively. The apparent $M_{\mathrm{n}}$ of P8a is higher than P7, it could be due to the poor solubility of P8a and also the presence of 
aggregation in its solution state. This reasons might lead to the overestimation of $M_{\mathrm{n}}$ for P8a.
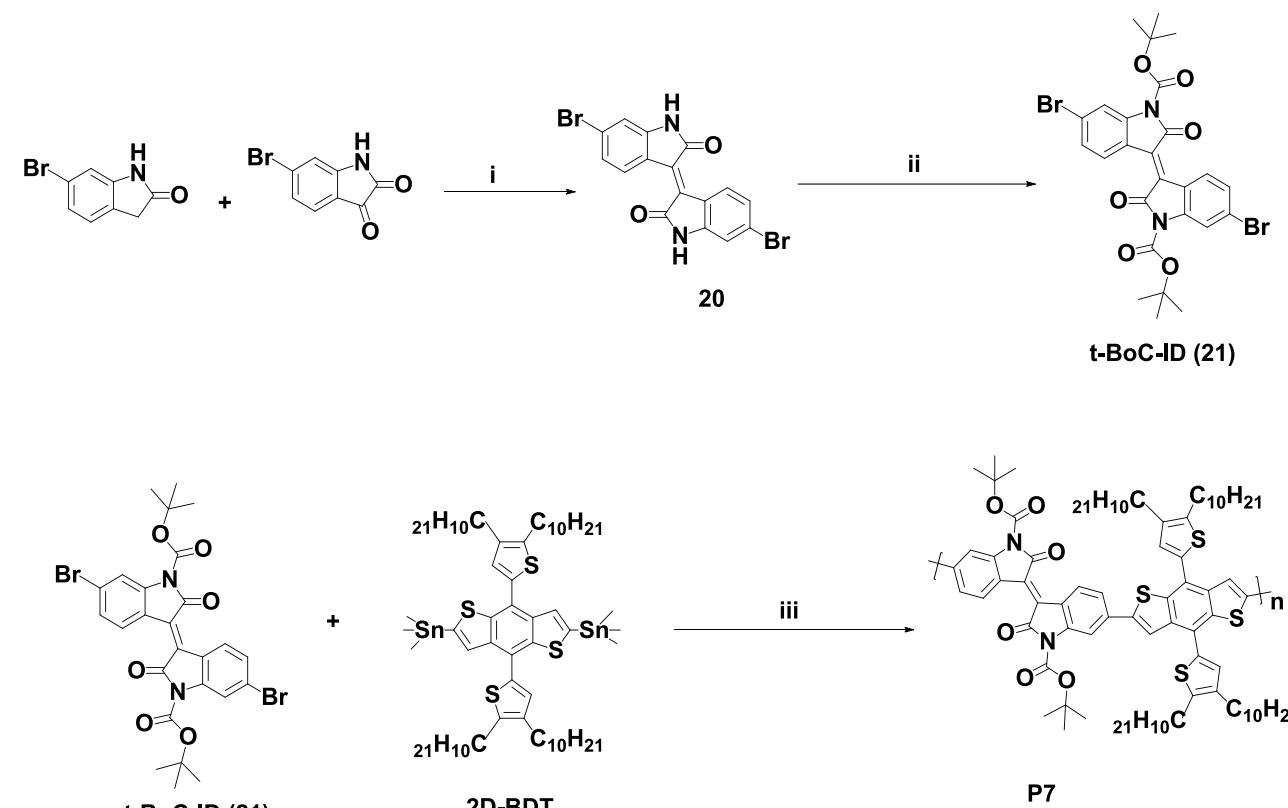

t-BoC-ID (21)
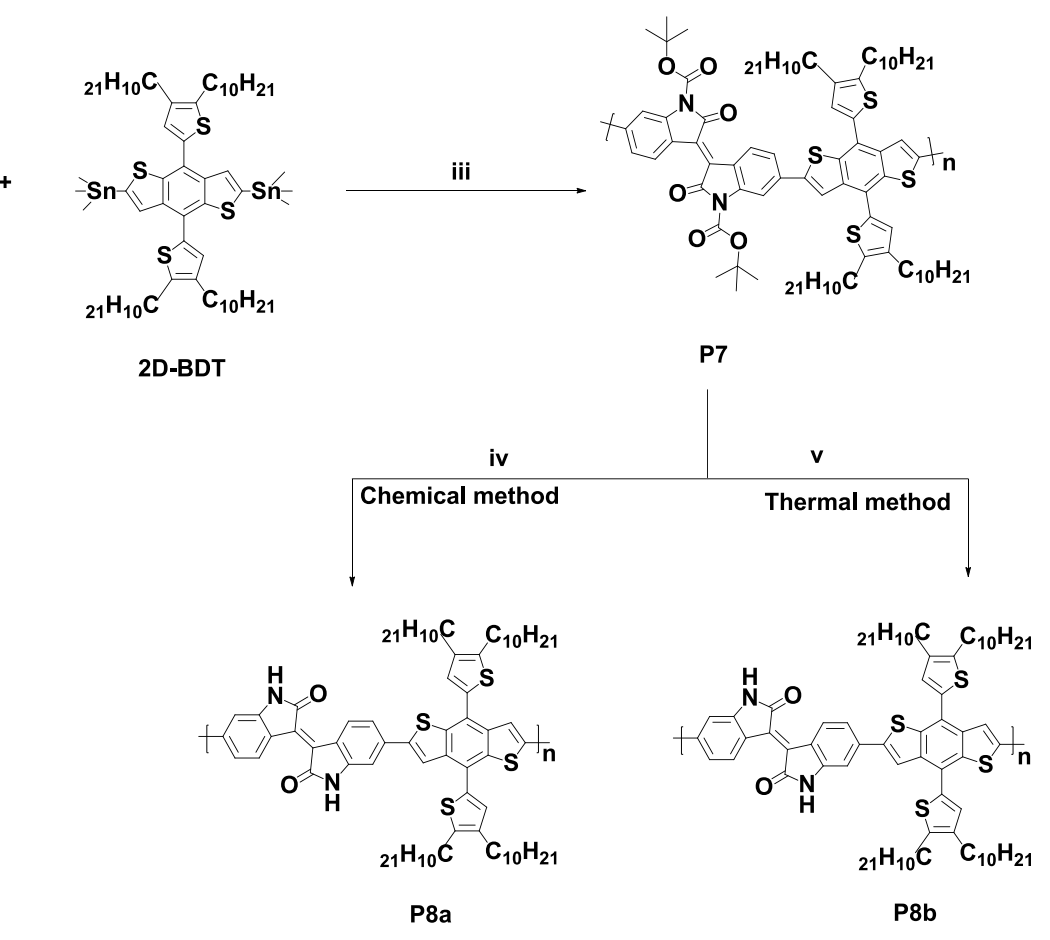

Scheme 8: Synthesis of the P7 polymer via Stille polymerization method and t-BoC deprotection by using chemical method (P8a) and thermal treatment method (P8b). (i) $\mathrm{AcOH}, \mathrm{HCl}$, reflux, $24 \mathrm{~h}, 100 \%$ yield. (ii) DMAP, $\mathrm{Et}_{3} \mathrm{~N}, \mathrm{BoC}_{2} \mathrm{O}, \mathrm{THF}, 24 \mathrm{~h}, 84 \%$ 
yield. (iii) $\mathrm{Pd}\left(\mathrm{PPh}_{3}\right)_{4}$, toluene, $85{ }^{\circ} \mathrm{C}, 48 \mathrm{~h}, 58.3 \%$ (P7) yield. (iv) $\mathrm{CF}_{3} \mathrm{COOH}, \mathrm{CH}_{2} \mathrm{Cl}_{2}$, R.T., 96.5\% (P8a) yield. (v) annealed at $200{ }^{\circ} \mathrm{C}$.

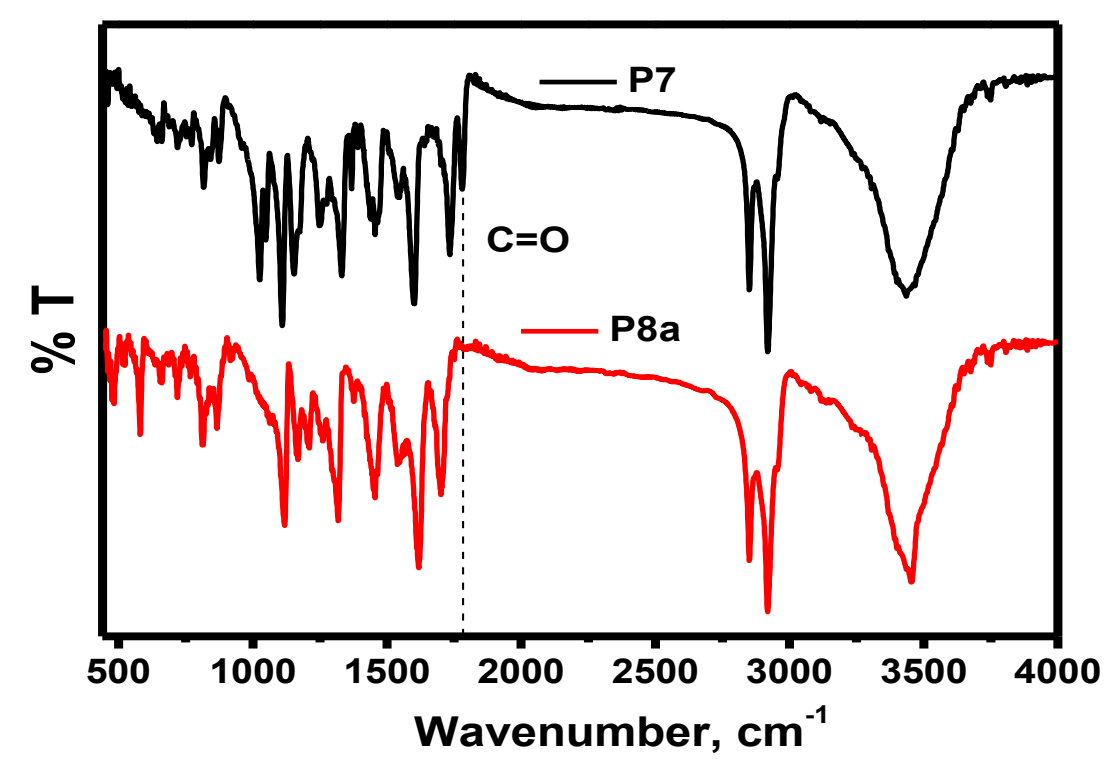

Figure 4.5. FT-IR spectra for $\mathbf{P 7}$ and P8a polymers.

\subsubsection{Thermal Properties}

Thermal stability of the polymers was investigated by using thermogravimetric analysis (TGA) with a heating rate of $10{ }^{\circ} \mathrm{C} \mathrm{min}^{-1}$ under nitrogen. Figure 4.6 shows TGA trace for P7 and P8a polymers, where a two-step thermal decomposition of tBoC protected polymer (P7) has been observed which indicates the elimination of t$\mathrm{BoC}$ protecting group from the backbone of the $\mathbf{P 7}$ polymer. As shown in Figure 4.6a, the weight loss occurred up to $83.3 \%$ might be from the total elimination of tBoC groups, which corresponds to $16.6 \%$ weight loss that is roughly in agreement with the calculated weight loss due to t-BoC group removal (14.6\%). The slight 
difference between the calculated and experimental weight loss might be due to the presence of some solvents. An additional step in the weight loss occurred at around $412{ }^{\circ} \mathrm{C}$ corresponds to the decomposition of the polymer backbone. Figure $4.6 \mathrm{~b}$ shows TGA for P8a where the onset decomposition temperature $\left(T_{\mathrm{d}}\right)$ with $5 \%$ weight loss is around $332{ }^{\circ} \mathrm{C}$. The thermal characteristics of the copolymers are summarized in Table 4.1.

Figure 4.6 (c) and (d) shows the differential scanning calorimetry (DSC) analysis of P7 and P8a polymers to investigate their thermal properties upon heating (endothermic) and cooling (exothermic) in the temperature range of 25 to $300{ }^{\circ} \mathrm{C}$. In Figure 4.6 (c), an endothermic transition was seen in the temperature range of 180 to $250{ }^{\circ} \mathrm{C}$ of the first heating cycle, which correspond to the thermal cleavage of the $\mathrm{t}$ BoC groups from the backbone of the $\mathbf{P 7}$ polymer. This is in agreement with the TGA analysis (see Figure 4.6 (a)). This broad endothermic transition has disappeared in the following heating cycle ( $2^{\text {nd }}$ heating cycle), indicating a complete elimination of t-BoC group from P7. The cooling (exothermic) cycles of P7 and the DSC trace for P8a didn't exhibit any change which corresponds to crystallization or molecular ordering. This indicates that both P7 and P8a were amorphous. 

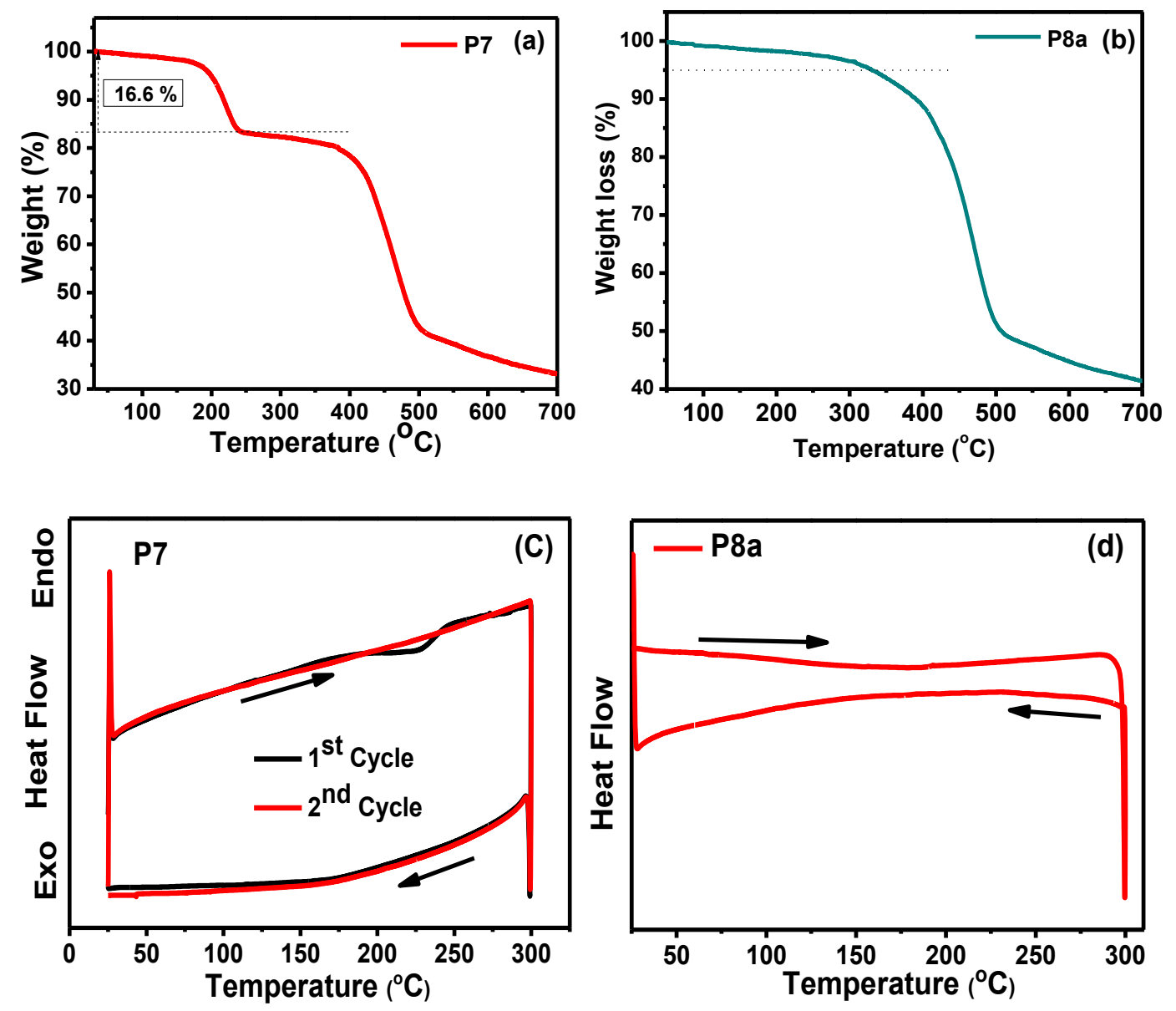

Figure 4.6. TGA trace for $\mathbf{P 7}$ (a) and $\mathbf{P 8 a}$ (b) polymers. DSC traces for $\mathbf{P 7}$ (c) and P8a (d) polymers with a heating rate of $10{ }^{\circ} \mathrm{C} \mathrm{min}^{-1}$ under nitrogen.

Table 4.1. Molecular weights and thermal properties of $\mathbf{P 7}$ and P8a copolymers.

\begin{tabular}{|l|l|l|l|l|l|l|}
\hline Polymer & $\begin{array}{l}\mathbf{a}_{\mathbf{n}} \\
\mathbf{k D a}\end{array}$ & $\begin{array}{l}\mathbf{a}_{\mathbf{w}} \\
\mathbf{k D a}\end{array}$ & aPDI & $\begin{array}{l}\mathbf{b} \mathbf{T}_{\mathbf{d}} \\
\left.\mathbf{(}^{\mathbf{}} \mathbf{C}\right)\end{array}$ & $\begin{array}{l}\mathbf{c} \text { Weight loss } \\
(\mathbf{\%})\end{array}$ & $\begin{array}{l}\text { dWeight loss } \\
(\mathbf{\%})\end{array}$ \\
\hline P7 & 17.7 & 26.1 & 1.47 & 202 & 16.6 & 14.6 \\
\hline P8a & 31.7 & 57.2 & 1.80 & 332 & - & - \\
\hline
\end{tabular}

${ }^{\text {a }}$ Determined by $\mathrm{GPC}$ at $80{ }^{\circ} \mathrm{C}$ in $\mathrm{CHCl}_{3}$ using polystyrene standards. ${ }^{\mathrm{b}} \mathrm{Decomposition}$ temperature, determined by TGA in nitrogen, based on 5\% weight loss. 
${ }^{c}$ Experimental weight loss of the t-BoC side groups. ${ }^{\mathrm{d}}$ Theoretical weight loss of the t-BoC side groups.

\subsubsection{Optical Properties}

Figure 4.7 shows absorption spectra of the polymers in dilute chloroform solution and spin-coated films on quartz substrates at $1500 \mathrm{rpm}$. Table 4.2 summarizes the optical data, including the maximum absorption peak wavelengths $\left(\lambda_{\max }\right)$, absorption edge wavelengths $\left(\lambda_{\text {onset }}\right)$, and the optical band gap $\left(\mathrm{E}_{\mathrm{g}}{ }^{\mathrm{opt}}\right)$ calculated from the $\lambda_{\text {onset }}$ of thin film measured at room temperature. Both solution and thin-films of P7 and P8a polymers exhibit wide absorption bands extending to $900 \mathrm{~nm}$. Absorption bands in the range of 300 to $450 \mathrm{~nm}$ correspond to localized $\pi$ $\pi *$ transition and from 500 to $900 \mathrm{~nm}$ corresponds to intermolecular charge transfer (ICT) between donor and acceptor units of the polymers.

P7 and P8a polymers in solution have displayed the $\lambda_{\max }\left(\lambda_{\text {onset }}\right)$ of 725 (857) nm and 692 (791) nm, respectively, as the t-BoC side group removed by $\mathrm{CF}_{3} \mathrm{COOH}$ treatment from the $\mathbf{P 7}$ polymer backbone, the absorption band shows blue-shift (hypochromism). It might be due to the change in conformation and coplanarity of the conjugation backbone. On the other hand, it could be caused by the change in the energy level of P8a frontier molecular orbitals, as t-BoC side group is an electron withdrawing group and thus its removal increases the bandgap of the polymer (see Table 4.3 from CV experiment). 
In order to investigate whether this blueshift is caused by aggregation in the solution, absorption spectra in chlorobenzene solution of P8a polymer were collected upon heating to $80^{\circ} \mathrm{C}$ and cooling to room temperature (see Figure 4.7 (c)), however, the change was insignificant. Therefore, it could be inferred, as there was no aggregation or presence of strong aggregation, which might be too strong to break at $80{ }^{\circ} \mathrm{C}$ in P8a solution. To our surprise, this hypochromism phenomenon was continued to appear even in their thin film absorbance spectra at room temperature. However, as P7 and P8a polymers annealed at $200{ }^{\circ} \mathrm{C}$, they exhibit redshift absorption spectra (bathochromism) in their $\lambda_{\max }\left(\lambda_{\text {onset }}\right) 731$ (880) $\mathrm{nm}$ and 702 (847) $\mathrm{nm}$ as compared to their respective absorption spectra in solution and film at room temperature. This could be due to the presence of an improved intermolecular interaction (formation of hydrogen bond network between $\mathrm{N}-\mathrm{H} \cdots \mathrm{O}=\mathrm{C}$ ) and thus their coplanarity. It is noteworthy to mention that, after annealing the thin film of the P7 polymer at $200{ }^{\circ} \mathrm{C}$, the t-BoC side group in the $\mathbf{P 7}$ polymer is expected to be eliminated. Thus, the resulting thin film could have a similar structure with P8a. This could give us a chance to compare the impact of t-BoC deprotection method (chemical vs. thermal) on the intermolecular interaction. Thus, P8b obtained via thermal treatment of $\mathbf{P 7}$ thin film at $200{ }^{\circ} \mathrm{C}$ shows a more redshift absorption spectra than P8a (obtained via acid treatment).

The observed difference between the thin film optical properties P8a and P8b polymers suggest that, an acid induced deprotection of the t-BoC group could cause 
the formation of more twisted conformation, less ordered amorphous phase and thus shorten the $\pi$-conjugation length and consequently blue shift the absorbance spectra than P8b polymer obtained via thermal treatment of P7 thin film. On the other hand, it could also be inferred based on the optical bandgap estimated from their respective $\lambda_{\text {onset }}$ of the thin film at room temperature 1.46 and $1.54 \mathrm{eV}$ for P7 and P8a polymers, respectively. The $\mathbf{P 8 b}$ optical bandgap could be estimated $\lambda_{\text {onset }}(800 \mathrm{~nm})$ is $1.4 \mathrm{eV}$, which is smaller than the other two polymers (P7 and P8a), thus the $\lambda_{\max }$ of P8b is at a longer wavelength.

Therefore, a thermal treatment method for $\mathrm{t}-\mathrm{BoC}$ removal is preferred to get red-shifted absorbance and then induce the required intermolecular interaction via hydrogen bond network formation. In addition to this, it could help to maintain solution processability to enable thin film casting.

Table 4.2. Optical data of the polymers, P7 and P8a.

\begin{tabular}{|c|c|c|c|c|c|c|c|}
\hline UV-vis & In CF & & Film & & $\begin{array}{l}\text { Film } \\
\text { (a) } 20\end{array}$ & ealed & $\begin{array}{c}{ }^{c} \mathbf{E}_{\mathrm{g}}{ }^{\mathrm{opt}} \\
(\mathrm{eV})\end{array}$ \\
\hline Polymer & $\begin{array}{l}{ }^{a} \lambda_{\text {max }} \\
{[\mathrm{nm}]}\end{array}$ & $\begin{array}{l}{ }^{b} \lambda_{\text {onset }} \\
{[\mathrm{nm}]}\end{array}$ & $\begin{array}{l}\lambda_{\text {max }} \\
{[\mathrm{nm}]}\end{array}$ & $\begin{array}{l}\mathbf{b}_{\text {onset }} \\
{[\mathrm{nm}]}\end{array}$ & $\begin{array}{l}{ }^{a} \lambda_{\max } \\
{[\mathrm{nm}]}\end{array}$ & $\begin{array}{l}\mathbf{b}_{\text {onset }} \\
{[\mathrm{nm}]}\end{array}$ & \\
\hline P7 & 725 & 857 & 716 & 847 & 731 & 880 & 1.46 \\
\hline P8a & 692 & 791 & 675 & 803 & 702 & 847 & 1.54 \\
\hline
\end{tabular}

${ }^{\mathrm{a}}$ Absorption maxima. ${ }^{\mathrm{b}}$ The onset absorption. ${ }^{\mathrm{c}}$ Estimated from the onset absorption in films at r.t. $\left(\lambda_{\text {onset }}\right): E_{\mathrm{g}}{ }^{\mathrm{opt}}(\mathrm{eV})=1240 / \lambda_{\text {onset }}[\mathrm{nm}]$ 

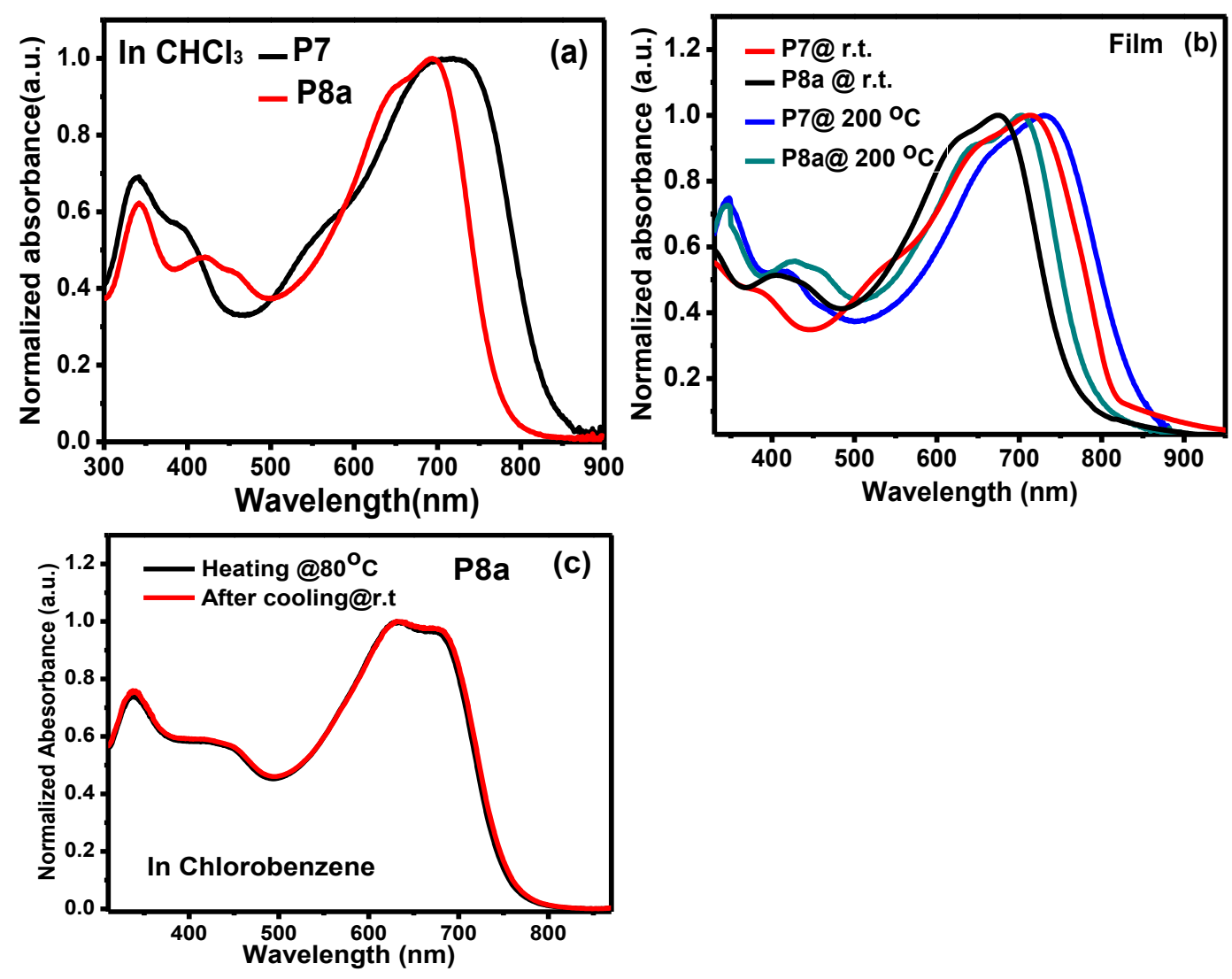

Figure 4.7. UV-vis spectra of $\mathbf{P 7}$ and P8a polymers in chloroform solution (a) and thin films (b). Chlorobenzene solution spectra of P8a at $80^{\circ} \mathrm{C}$ and after cooling to r.t (c).

\subsubsection{Electrochemical Properties}

As shown in Table 3, the HOMO ${ }^{\text {ele }} / \mathrm{LUMO}^{\text {ele }}$ energy levels of P7 and P8a are $-5.26 /-3.72$ and $-5.22 /-3.71 \mathrm{eV}$, respectively. Thus, their band gaps measured from $\mathrm{CV}\left(\mathrm{E}_{\mathrm{g}}{ }^{\mathrm{elc}}\right)$ are 1.54 and $1.51 \mathrm{eV}$, respectively. These electrochemical band gaps are slightly different from those on the basis of absorption edges data but can be accepted 
within the experimental error. The HOMOs ${ }^{\text {ele }}$ and LUMOs ${ }^{\text {ele }}$ energy level variation between these two polymers is not significant, but when LUMOs ${ }^{\text {opt }}$ is taken into consideration, there is an obvious variation matching with the theoretical expectation. Which is after t-BoC side group elimination, the LUMO ${ }^{\text {opt }}$ level has shown an increment from -3.80 to $-3.68 \mathrm{eV}$ because t-BoC groups have an electron withdrawing effect. Whereas, their HOMO level didn’t show any significant change because their donor unit is the same, which mainly determines the position of HOMO energy level. These results are in agreement with the hypochromism phenomenon observed after t-BoC elimination.
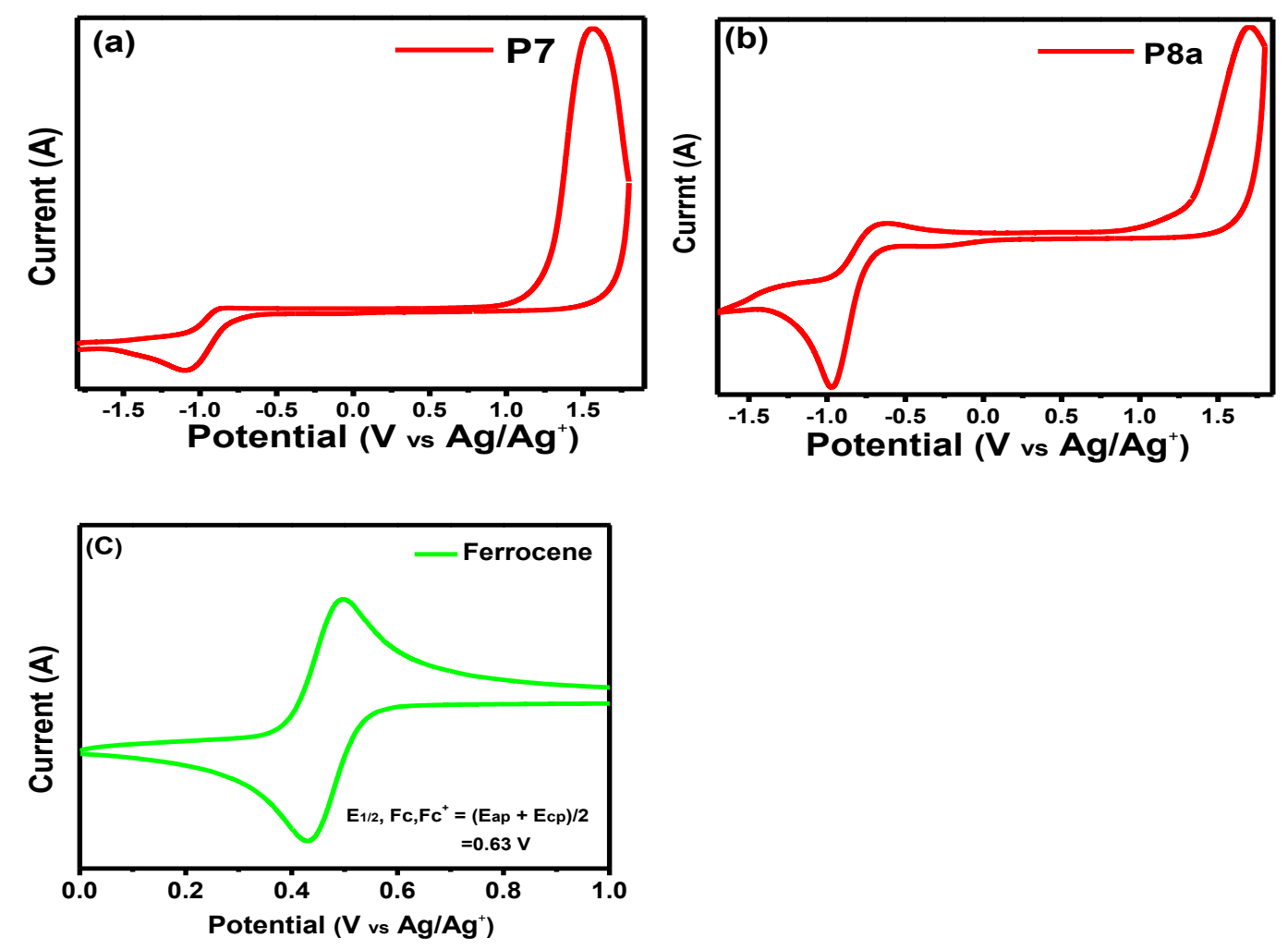

Figure 4.8. The cyclic voltammograms of P7 (a), P8a (b), and Ferrocene (c) films 
on a glassy carbon working electrode measured in $0.1 \mathrm{~mol} / \mathrm{lit}_{\mathrm{Bu}} \mathrm{NPF}_{6}$ acetonitrile solutions at a scan rate of $50 \mathrm{mVs}^{-1}$.

Table 4.3. Electrochemical properties of polymers.

\begin{tabular}{|c|c|c|c|c|c|c|c|}
\hline $\begin{array}{l}\text { Poly } \\
\text { mer }\end{array}$ & $\begin{array}{l}\text { Eox }_{\text {onset }} \\
(\mathbf{V})\end{array}$ & $\begin{array}{l}\text { Ered }^{\text {onset }} \\
(\mathrm{V})\end{array}$ & $\begin{array}{l}{ }^{\text {a} H O M O} \\
(\mathrm{eV})\end{array}$ & $\begin{array}{l}{ }^{\text {a }} L U M O O^{\text {ele }} \\
(\mathbf{e V})\end{array}$ & $\begin{array}{l}{ }^{\text {bLUMO }}+{ }^{o p t} \\
(e V)\end{array}$ & $\begin{array}{l}{ }^{\mathbf{c}} \mathbf{E}_{\mathbf{g}} \text { ele } \\
(\mathbf{e V})\end{array}$ & $\begin{array}{l}{ }^{c} E_{g}{ }^{o p t} \\
(e V)\end{array}$ \\
\hline P7 & 0.93 & -0.61 & -5.26 & -3.72 & -3.80 & 1.54 & 1.46 \\
\hline P8a & 0.89 & -0.62 & -5.22 & -3.71 & -3.68 & 1.51 & 1.54 \\
\hline
\end{tabular}

\subsubsection{Field-Effect Transistor Devices}

Bottom gate/top contact OFET devices were fabricated in air to investigate the effect of t-BoC and its deprotection on the P7 polymer. Figure 4.9 illustrated the output and transfer characteristics of OFETs of P7 as the semiconducting layer. The hole mobility increased from $5.2 \times 10^{-5}$ to $9.7 \times 10^{-5} \mathrm{~cm}^{2} \mathrm{~V}^{-1} \mathrm{~s}^{-1}$ upon annealing from r.t to $100{ }^{\circ} \mathrm{C}$, where the t-BoC side group is not expected completely removed as we can see it from its TGA curve (see Figure 4.6 (a)). Whereas it shows a slight decrease in its hole mobility, $8.3 \times 10^{-5} \mathrm{~cm}^{2} \mathrm{~V}^{-1} \mathrm{~s}^{-1}$. As the annealing temperature reached to $150{ }^{\circ} \mathrm{C}$, and it continued to decrease to $7.4 \times 10^{-5} \mathrm{~cm}^{2} \mathrm{~V}^{-1} \mathrm{~s}^{-1}$. From this result (see Table 4.4) one can see the $\mu_{\mathrm{h}}$ of $\mathbf{P 7}$ at $200{ }^{\circ} \mathrm{C}$ is still higher than that of r.t. $\mu_{\mathrm{h}}$. This phenomenon was also observed for DPP-based polymer containing thermal-labile 
group and have described the reason could be the twist in the backbone of the conjugated polymer after annealing at $200{ }^{\circ} \mathrm{C}$ and consequently decreases the $\pi$ conjugation length and also the formation of less ordered (amorphous) structures in the solid state. ${ }^{273}$

The $\mu_{\mathrm{h}}$ results obtained in this work are comparable with the report made by Liu et al. ${ }^{260}$ for structurally similar t-BoC-isoindigo based conjugated polymer even if it was fabricated in the nitrogen-protected glove box. In addition to this, the low mobility could be understood from a slightly larger hole injection barrier caused by the lower HOMO energy level of $\mathbf{P 7}$ with respect to gold (source electrode), which has a work function of $-5.1 \mathrm{eV}$. On the other hand, the increase in hole mobility upon annealing to $200{ }^{\circ} \mathrm{C}$, could be due to a slight increase in the HOMO energy level as the t-BoC side group gets eliminated and thus the hole-injection from gold contact to $\mathbf{P 7}$ polymer semiconductor could be enhanced. ${ }^{202}$ In addition to this, the improved hole mobility after thermal elimination of t-BoC groups may be correlated with an improved coplanarity, which can lead to increased intermolecular stacking and improved charge carrier transport. It is worth to mention that OFET device based on P8a has not been fabricated due to its poor solubility and poor film quality.

Table 4.4. Performance characteristics of OFET devices for $\mathbf{P 7}$ polymer semiconductor on an OTS-18-treatd substrate fabricated and measured ambient conditions. 


\begin{tabular}{|l|l|l|l|}
\hline P7 Annealing & $\boldsymbol{\mu}_{\mathbf{h}, \mathbf{a v g}}$ & $\mathbf{V}_{\text {th }}$ & $\mathbf{I}_{\text {on/off }}$ \\
temperature $\left.\mathbf{(}^{\mathbf{0}} \mathbf{C}\right)$ & $\left(\mathbf{c m}^{\mathbf{2}} \mathbf{V}^{\mathbf{- 1}} \mathbf{s}^{\mathbf{- 1}}\right)$ & $\mathbf{V})$ & ratio \\
\hline R.T. & $5.2 \times 10^{-5}$ & 3 & $10^{3}$ \\
\hline $\mathbf{1 0 0}$ & $9.7 \times 10^{-5}$ & -1 & $10^{3}-10^{4}$ \\
\hline $\mathbf{1 5 0}$ & $8.3 \times 10^{-5}$ & 1 & $10^{3}-10^{4}$ \\
\hline $\mathbf{2 0 0}$ & $7.4 \times 10^{-5}$ & 15 & $10^{3}$ \\
\hline
\end{tabular}
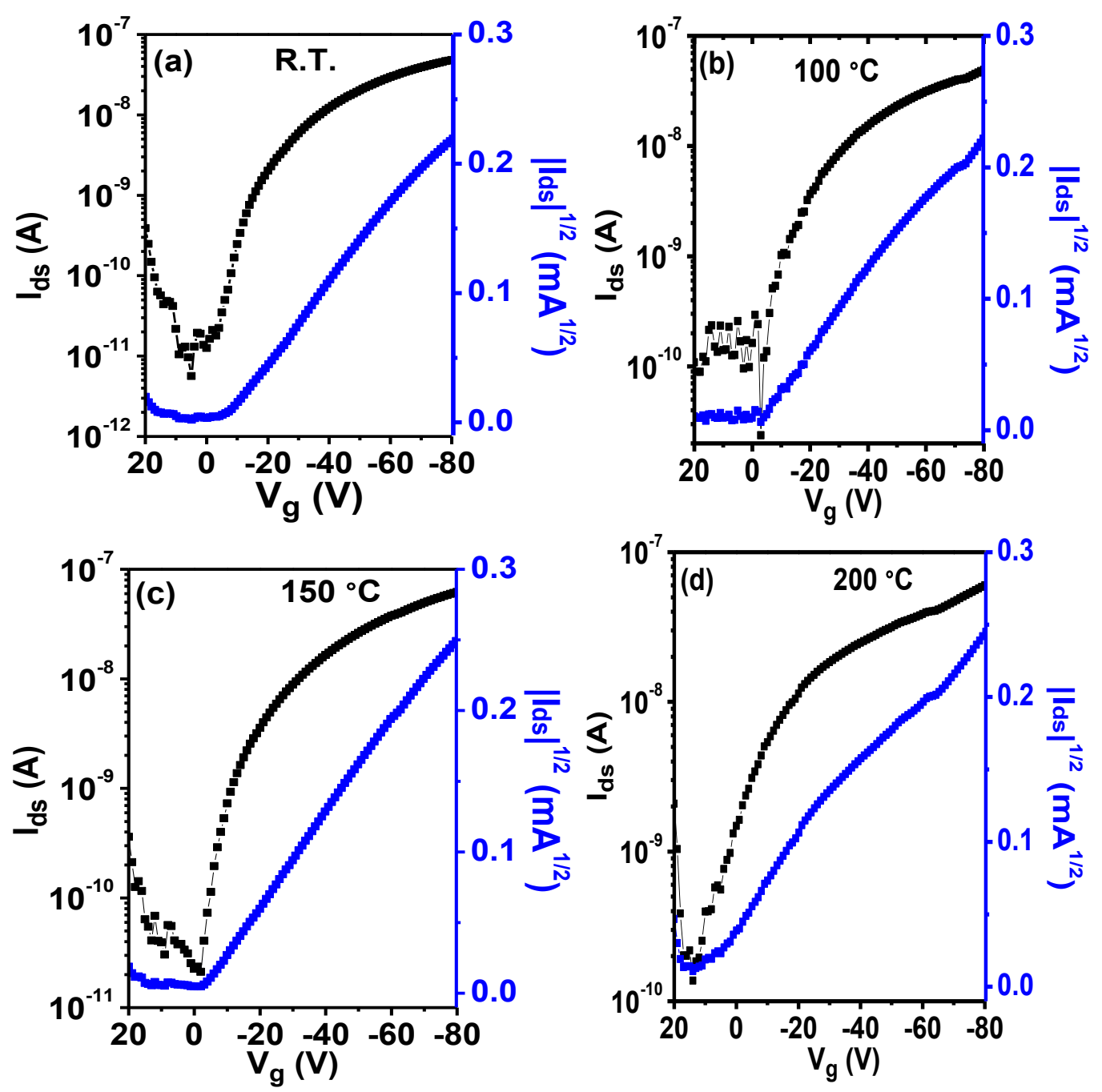

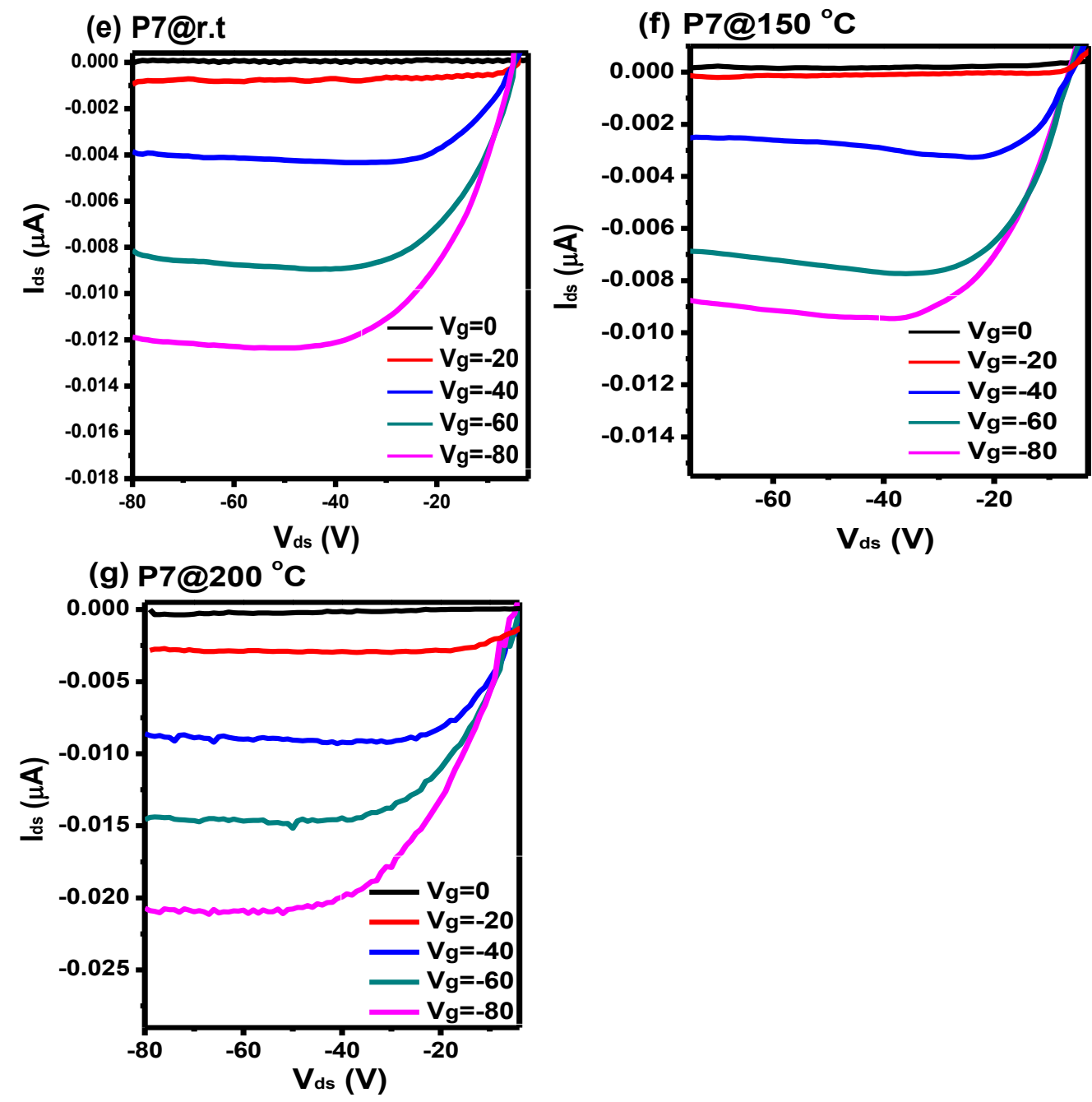

Figure 4.9. Typical transfer curves (a to d) and output characteristics curves (e to g) for the P7 polymer.

\subsection{Conclusion}

In summary, a D-A conjugated polymer comprising tert-butoxycarbonyl (tBoc) substituted isoindigo acceptor moiety was designed, synthesized, and characterized. The impact of t-BoC latent side group deprotection method (chemical 
vs. thermal) on the physicochemical characteristics was studied. Therefore, removing t-BoC side group via chemical method (treatment with $\mathrm{CF}_{3} \mathrm{COOH}$ ) gives less redshifted absorption spectra as compared with the thermal treatment method of its spincast thin film. In addition to this, removing the t-BoC group has greatly reduced the solubility of the polymers and thus affected film formation by spin cast processing. Therefore, OFETs based on the thermally treated thin film of the $\mathbf{P 7}$ polymer has given high hole mobility of $9.7 \times 10^{-5} \mathrm{~cm}^{2} \mathrm{~V}^{-1} \mathrm{~s}^{-1}$ under ambient condition. 


\section{CHAPTER 5}

\section{Direct C-H Arylation Polymerization for Efficient}

\section{Synthesis of 5,6-Difluoro-2,1,3-Benzothiadiazole}

\section{Based Donor-Acceptor Conjugated polymer}

\subsection{Introduction}

Conjugated organic semiconductors have interesting properties like solution processability which could enable cheap and flexible organic electronic devices such as organic field-effect transistors (OFETs) $)^{239-244}$, organic photovoltaics (OPVs) $)^{237,245-}$ ${ }^{249}$, and organic light emitting diodes (OLEDs). ${ }^{250,251}$ Conventionally, $\pi$-conjugated polymers are synthesized via transition metal-catalyzed polycondensation reactions, such as Suzuki-Miyaura and Migita-Kosugi-Stille couplings. ${ }^{274-277}$ Despite their great versatility and choice in the synthesis of conjugated polymers, these protocols require the preparation of high-purity organometallic functionalized monomers such as arylene diboronic acid/ diboronic ester (for Suzuki-type coupling) and distannyl arylenes (for Stille-type coupling) using flammable and toxic precursors. In addition, these polycondensation reactions could produce a stoichiometric amount of toxic byproducts such as trialkyltin compound, which would become a key issue in the 
future commercialization of conjugated polymers for organic electronics applications. Recently, a new coupling chemistry called direct $(\mathrm{C}-\mathrm{H})$ arylation polymerization (DAP) have attracted a tremendous attention in $\pi$-conjugated polymers synthesis, because it involves the coupling of an aryl halide monomer directly with another arene monomer without the need for preactivation of $\mathrm{C}-\mathrm{H}$ bonds. ${ }^{278-280}$ Therefore, DAP is an economically efficient and environmentally benign approach compared with the traditional coupling methods (such as Stille and Suzuki couplings). ${ }^{61,281-284}$

5,6-Difluoro-2,1,3-benzothiadiazole (DFBT) has been sought as an attractive building block for the construction of $\pi$-conjugated polymers than its non-fluorinated analog. ${ }^{249,285-288}$ The electron-withdrawing nature of fluorine substituents in the conjugated backbone of the polymer resulted in lowering the HOMO energy level and consequently could enhance open circuit voltage $\left(\mathrm{V}_{\mathrm{oc}}\right)$ of bulk heterojunction (BHJ) solar cells. ${ }^{285,289-291}$ In addition, fluorination of 2,1,3-benzothiadiazole could improve planarity of the polymer backbone and therefore, effective $\pi$-conjugation length of the polymer. ${ }^{292}$ However, most of the DFBT based conjugated polymers are synthesized by using traditional coupling techniques (like Stille and Suzuki couplings) but there are a few reports are available on their synthesis based on an emerging DAP coupling method. For instance, Marder and coworkers have reported the synthesis of good quality 4,7-diaryl-5,6-difluoro-2,1,3-benzothiadiazole based small molecules. Wang and coworkers have reported the synthesis of high molecular 
weight poly(5,6-difluoro-2,1,3-benzothiadiazole-alt-9,9-dioctylfluorene) using $\mathrm{Pd}(\mathrm{OAc})_{2}$ as a catalyst via DAP. ${ }^{293-295}$

This Chapter presents the synthesis of DFBT based D-A conjugated polymer, poly(5,6-difluoro-2,1,3-benzothiadiazole-alt-quaternary thiophene( $\left(\mathrm{Th}_{4}\right)$ ) PDFBTTh4 via direct arylation polymerization (DAP). Recently, the synthesis of the PDFBT-Th 4 polymer has been reported via a traditional coupling method (Stille polymerization) and has shown high PCE of more than $10 \%$ for the first time in single-junction OPVs and a hole mobility of $1.92 \mathrm{~cm}^{2} \mathrm{~V}^{-1} \mathrm{~s}^{-1}$ in OFETs. ${ }^{296,129}$ As Stille coupling involves a tedious preactivation of $\mathrm{C}-\mathrm{H}$ bonds with a toxic organo-thin reagent, thus it is not economically and environmentally recommended a method for its future large-scale production and commercialization for organic electronics applications. In addition to this, researchers have indicated that organometallic end groups in the polymer usually deteriorate the performance of the polymer their corresponding organic electronic devices. ${ }^{297-301}$ Therefore, we anticipated exploring the synthesis of this interesting polymer, PDFBT-Th4 by using an emerging synthesis method, direct arylation polymerization (DAP) to give an additional insight for its synthesis. 




Scheme 9: The synthesis of PDFTBT-Th4 via DAP and Stille polymerization methods. The synthesis of PDFTBT-Th4 via DAP includes the application of small molecule direct arylation to generate one of the monomers. This enables the synthesis of DAP PDFTBT-Th4 in only 3 steps while the synthesis of Stille PDFTBT-Th4 requires 4 steps. Conditions are as follows: (i) $\mathrm{Pd}_{2}(\mathrm{dba})_{3}$, ((o- $\left.\mathrm{MeOPh}\right)_{3} \mathrm{P}, \mathrm{K}_{2} \mathrm{CO}_{3}$, PivOH, o-xylene, 61.0\% yield for (compound 22); (ii) NBS, THF, 89\% yield for (compound 23); (iii) n-BuLi, THF, $-78{ }^{\circ} \mathrm{C}, \mathrm{SnMe}_{3} \mathrm{Cl}$; (iv) DAP Protocol (See Table 5.1); (v) $\mathrm{P}(\mathrm{o}-\text { tol })_{3}, \mathrm{Pd}_{2}(\mathrm{dba})_{3}$, o-xylene, $56 \%$ yield. 


\subsection{Experimental}

Materials. Unless stated otherwise, all chemicals and solvents were obtained from commercial sources and used without further purification. 4,7-dibromo-5,6difluorobenzo[c][1,2,5]thiadiazole (DBrDFBT) were obtained from Derthon optoelectronics materials and 3-(2-octyldodecyl) thiophene were prepared according to the reported procedures. ${ }^{185,302}$

Photovoltaic Device fabrication and characterization. A pre-patterned ITOcoated glass substrate (sheet resistance of $\sim 15 \Omega$ /square) was used as a substrate. It was cleaned by sequential sonications in soap DI water, DI water, acetone and isopropanol for $15 \mathrm{~min}$ at each step. After ultraviolet/ozone treatment for $15 \mathrm{~min}$, a $\mathrm{ZnO}$ as an electron-transporting layer was spin coated at 2,500 rpm for $1 \mathrm{~min}$ and then it was backed at $200{ }^{\circ} \mathrm{C}$ for $1 \mathrm{~h}$. The active layer solutions (PDFBT-Th4: $\mathrm{PC}_{71} \mathrm{BM}$ in ratio of 1:1.2) with $9 \mathrm{mg} \mathrm{mL}^{-1}$ concentration of PDFBT-Th4 polymer were prepared in chlorobenzene: 1,2-dichlorobenzene $(1: 1 \mathrm{v} / \mathrm{v})$ with $3 \%$ of $1,8-$ diiodooctane (DIO) as an additive. Before spin coating, the active layer, both the active layer solution and ITO substrate were preheated on a hot plate at $110{ }^{\circ} \mathrm{C}$ for 5 min. The warm solution of the active layer was spin coated on the preheated substrate at $800 \mathrm{rpm}$. The polymer/fullerene films were then annealed at $80{ }^{\circ} \mathrm{C}$ for $10 \mathrm{~min}$ before being transferred to the vacuum chamber of a thermal evaporator. A thin layer of $\mathrm{MoO}_{3}(10 \mathrm{~nm})$ was deposited as the anode interlayer, followed by deposition of $\mathrm{Ag}(100 \mathrm{~nm})$ as the top electrode. Device current $(\mathrm{J})$ - voltage $(\mathrm{V})$ characteristics 
was measured under AM1.5G $\left(100 \mathrm{~mW} \mathrm{~cm}^{-2}\right)$ solar simulator. $\mathrm{J}-\mathrm{V}$ characteristics were recorded using a Keithley SCS-4200 Semiconductor parameter analyzer. All OPVs fabrication and measurement were done in the air without any protective atmosphere.

\subsubsection{General synthesis procedure of Compound 22 via direct $\mathrm{C}-\mathrm{H}$ arylation couplings}

\section{4,7-Bis(4-(2-octyldodecyl)thiophen-2-yl)-5,6-difluoro[2,1,3]benzothiadiazole} (22)

4,7-dibromo -5,6-difluorobenzo[c][1,2,5]thiadiazole (DBrDFBT) (0.4 mmol, $131.98 \mathrm{mg}), 3$-(2-octyldodecyl) thiophene (Compound 3), $\mathrm{Pd}_{2}(\mathrm{dba})_{3}(20 \mu \mathrm{mol}, 18$ $\mathrm{mg})$, tris(2-methoxyphenyl)phosphine ((o-MeOPh) $\left.{ }_{3} \mathrm{P}, 40 \mu \mathrm{mol}, 14 \mathrm{mg}\right)$, potassium carbonate $\left(\mathrm{K}_{2} \mathrm{CO}_{3}, 1.4 \mathrm{mmol}, 193 \mathrm{mg}\right)$, pivalic acid (PivOH, $\left.0.12 \mathrm{mmol}, 12 \mathrm{mg}\right)$, and 1,2-dimethylbenzene (o-xylene, $2 \mathrm{~mL}$ ) were added under a nitrogen atmosphere in a reaction vial with a magnetic stirring bar. The vial was sealed with an aluminum cap with PTFE/silicone septum and then heated in a preheated oil bath for $24 \mathrm{~h}$. After being cooled to room temperature, the reaction mixture was diluted with chloroform and then filtered to remove the insoluble species. The filtrate was concentrated and purified by column chromatography with hexane as an eluent.

Entry 1: 3-(2-octyldodecyl)thiophene (2.92 g, $8 \mathrm{mmol}, 20$ equiv.) an orange color oil, to give $200 \mathrm{mg}$ of Compound 22 in $55.7 \%$ yield. 
${ }^{1} \mathrm{H}-\mathrm{NMR}\left(300 \mathrm{MHz}, \mathrm{CDCl}_{3}\right) \delta$ (ppm): 8.09 (s, 2H), 7.18 (s, 2H), 2.66 (d, $J=6.9$ $\mathrm{Hz}, 4 \mathrm{H}), 1.69$ (s, 2H), 1.56-1.25 (m, 80H), 0.87 (t, $J=12.9 \mathrm{~Hz}, 12 \mathrm{H})$.

Entry 2: 3-(2-octyldodecyl)thiophene (1.46 g, $4 \mathrm{mmol}, 10$ equiv.) an orange color oil, to give $220 \mathrm{mg}$ of Compound 22 in $61.0 \%$ yield.

${ }^{1} \mathrm{H}-\mathrm{NMR}\left(300 \mathrm{MHz}, \mathrm{CDCl}_{3}\right) \delta(\mathrm{ppm}): 8.09$ (s, 2H), 7.18 (s, 2H), 2.66 (d, $J=6.9$ $\mathrm{Hz}, 4 \mathrm{H}), 1.69$ (s, 2H), 1.56-1.25 (m, 80H), 0.87 (t, $J=12.9 \mathrm{~Hz}, 12 \mathrm{H})$.

${ }^{13} \mathrm{C}$ NMR (300 MHz, $\left.\mathrm{CDCl}_{3}\right) \delta(\mathrm{ppm}): 151.5,151.3,148.9,148.8,148.1,147.8$, 142.2, 132.7, 131.0, 124.8, 111.6, 111.6, 111.5, 111.5, 39.0, 34.8, 33.3, 32.0, 30.1, 29.7, 29.7, 29.4, 26.7, 22.7, 14.1.

\section{4,7-Bis(5-bromo-4-(2-octyldodecyl)thiophen-2-yl)-5,6-difluoro[2,1,3]benzothia diazole (23)}

N-Bromosuccinimide $(92.71 \mathrm{mg}, 0.521 \mathrm{mmol})$ was added in portion to a solution of 4,7-bis(4-(2-octyldodecyl)thiophen-2-yl)-5,6-difluoro[2,1,3]benzothia diazole (22) (220 mg, $0.245 \mathrm{mmol})$ in $10 \mathrm{~mL}$ of THF at room temperature. After the mixture was stirred for $24 \mathrm{~h}, 50 \mathrm{~mL}$ water was added and the mixture was extracted with dichloromethane for three times. The organic phases were combined, washed with brine, and dried over anhydrous $\mathrm{MgSO}_{4}$. The filtrate was concentrated and purified by column chromatography with hexane as an eluent to afford $230 \mathrm{mg}$ orange solid in $89 \%$ yield. 
${ }^{1} \mathrm{H}-\mathrm{NMR}\left(300 \mathrm{MHz}, \mathrm{CDCl}_{3}\right) \delta$ (ppm): $7.93(\mathrm{~s}, 2 \mathrm{H}), 2.60(\mathrm{~d}, J=7.0 \mathrm{~Hz}, 4 \mathrm{H})$, $1.74(\mathrm{~s}, 2 \mathrm{H}), 1.25(\mathrm{~m}, 80 \mathrm{H}), 0.86(\mathrm{t}, J=12 \mathrm{~Hz}, 12 \mathrm{H})$.

\subsubsection{General synthesis procedure for PDFBT-Th 4 polymer via direct arylation polymerization (DAP)}

A mixture of 4,7-Bis(5-bromo-4-(2-octyldodecyl)thiophen-2-yl)-5,6difluoro[2,1,3] benzothiadiazole (23) (1 equiv.) and 2,2'-bithiophene (BT) (1equiv.), palladium source, ligand, base, $\mathrm{PivOH}$, and solvent $(0.2 \mathrm{M})$ was added in a reaction vial containing a magnetic stirring bar under nitrogen atmosphere. The vial sealed with an aluminum cap with PTFE/silicone septum and then heated in a preheated oil bath at $100{ }^{\circ} \mathrm{C}$ except for Entry $\mathbf{E 2}$ heated at $140{ }^{\circ} \mathrm{C}$. After being cooled to room temperature, the reaction mixture was diluted with $\mathrm{CHCl}_{3}$ and added dropwise to 150 $\mathrm{mL}$ of methanol, precipitates were collected by filtration and then subjected to Soxhlet extraction with methanol, acetone, hexane, and chloroform sequentially each for $24 \mathrm{~h}$. The hexane and/or chloroform fraction was concentrated and precipitated into methanol. The precipitates were collected by filtration and dried under vacuum before further characterization.

Entry E1: $\mathrm{Pd}_{2}(\mathrm{dba})_{3}(5 \mathrm{~mol} \%),(o-\mathrm{MeOPh})_{3} \mathrm{P}(10 \mathrm{~mol} \%), \mathrm{K}_{2} \mathrm{CO}_{3}$ (4 equiv.), PivOH (50 mol\%), and o-xylene solvent $(0.2 \mathrm{M})$ at $100{ }^{\circ} \mathrm{C}$. Yield: $11 \%$ from $\mathrm{CHCl}_{3}$ fraction. 
Entry E2: $\mathrm{Pd}_{2}(\mathrm{dba})_{3}(5 \mathrm{~mol} \%),(o-\mathrm{MeOPh})_{3} \mathrm{P}(10 \mathrm{~mol} \%), \mathrm{K}_{2} \mathrm{CO}_{3}$ (4 equiv.), $\mathrm{PivOH}(50 \mathrm{~mol} \%)$, and o-xylene solvent $(0.2 \mathrm{M})$ at $140{ }^{\circ} \mathrm{C}$. Yield: $14 \%$ from $\mathrm{CHCl}_{3}$ fraction.

Entry 3a: $\mathrm{Pd}(\mathrm{OAc})_{2}(5 \mathrm{~mol} \%),(o-\mathrm{MeOPh})_{3} \mathrm{P}(10 \mathrm{~mol} \%), \mathrm{K}_{2} \mathrm{CO}_{3}$ (4 equiv.), $\mathrm{PivOH}(50 \% \mathrm{~mol})$, and o-xylene solvent $(0.2 \mathrm{M})$ at $100{ }^{\circ} \mathrm{C}$ with reaction time of $(72$ h, see Table 5.1). Yield: $81 \%$ from $\mathrm{CHCl}_{3}$ fraction.

Entry 3b: $\mathrm{Pd}(\mathrm{OAc})_{2}(5 \mathrm{~mol} \%),(o-\mathrm{MeOPh})_{3} \mathrm{P}(10 \mathrm{~mol} \%), \mathrm{K}_{2} \mathrm{CO}_{3}$ (4 equiv.), $\mathrm{PivOH}(50 \% \mathrm{~mol})$, and o-xylene solvent $(0.2 \mathrm{M})$ at $100{ }^{\circ} \mathrm{C}$ with reaction time of $(48$ h, see Table 5.1). Yield: $83 \%$ from hexane fraction.

Entry 3c: $\mathrm{Pd}(\mathrm{OAc})_{2}(5 \mathrm{~mol} \%),(o-\mathrm{MeOPh})_{3} \mathrm{P}(10 \mathrm{~mol} \%), \mathrm{K}_{2} \mathrm{CO}_{3}$ (4 equiv.), PivOH (50\% mol), and o-xylene solvent $(0.2 \mathrm{M})$ at $100{ }^{\circ} \mathrm{C}$ with reaction time of $(24$ h, see Table 5.1). Yield: $85 \%$ from hexane fraction.

Entry E4: $\mathrm{Pd}(\mathrm{OAc})_{2}(5 \mathrm{~mol} \%), \mathrm{PCy}_{3} . \mathrm{HBF}_{4}(10 \mathrm{~mol} \%), \mathrm{K}_{2} \mathrm{CO}_{3}$ (4 equiv.), PivOH (50 mol\%), and o-xylene solvent $(0.2 \mathrm{M})$ at $100{ }^{\circ} \mathrm{C}$. no polymerization.

Entry E5: $\mathrm{Pd}(\mathrm{OAc})_{2}(5 \mathrm{~mol} \%), \mathrm{PCy}_{3} . \mathrm{HBF}_{4}(10 \mathrm{~mol} \%), \mathrm{Cs}_{2} \mathrm{CO}_{3}$ (4 equiv.), PivOH (50 mol\%), and o-xylene solvent $(0.2 \mathrm{M})$ at $100{ }^{\circ} \mathrm{C}$. Yield: $81 \%$ from hexane fraction.

Entry E6: Herrmann's catalyst (5 mol\%), (o-MeOPh) $)_{3} \mathrm{P}(10 \mathrm{~mol} \%), \mathrm{K}_{2} \mathrm{CO}_{3}(4$ equiv.), $\mathrm{PivOH}(50 \mathrm{~mol} \%)$, and o-xylene solvent $(0.2 \mathrm{M})$ at $100{ }^{\circ} \mathrm{C}$. Yield: $67 \%$ from 
hexane fraction.

Entry E7: $\mathrm{Pd}_{2}(\mathrm{dba})_{3}(5 \mathrm{~mol} \%),(o-\mathrm{MeOPh})_{3} \mathrm{P}(10 \mathrm{~mol} \%), \mathrm{K}_{2} \mathrm{CO}_{3}$ (4 equiv.), PivOH (50 mol\%), and DMAc solvent $(0.2 \mathrm{M})$ at $100{ }^{\circ} \mathrm{C}$. no polymerization.

Entry E8: $\mathrm{Pd}_{2}(\mathrm{dba})_{3}(5 \mathrm{~mol} \%),(o-\mathrm{MeOPh})_{3} \mathrm{P}(10 \mathrm{~mol} \%), \mathrm{K}_{2} \mathrm{CO}_{3}$ (4 equiv.), PivOH (50 mol\%), and chlorobenzene solvent $(0.2 \mathrm{M})$ at $100{ }^{\circ} \mathrm{C}$. Yield: $79 \%$ from hexane fraction.

\subsubsection{Synthesis of PDFBT-Th 4 polymer via Stille polymerization}

A mixture of 4,7-Bis(5-bromo-4-(2-octyldodecyl)thiophen-2-yl)-5,6difluoro[2,1,3] benzothiadiazole (23) (80 mg, $0.0758 \mathrm{mmol}, 1$ equiv.), 5,5'bis(trimethylstannyl)-2,2'-bithiophene (37.29 mg, $0.0758 \mathrm{mmol}, 1$ equiv.), $\mathrm{Pd}_{2}(\mathrm{dba})_{3}$ (3.28 mol\%, $2.27 \mathrm{mg})$, and (o-tolyl) ${ }_{3} \mathrm{P}(19.7 \mathrm{~mol} \%, 4.55 \mathrm{mg})$ were added to a $50 \mathrm{~mL}$ flask under nitrogen atmosphere. After three cycles of degassing and purging with nitrogen $3 \mathrm{~mL}$ of anhydrous o-xylene was added and then the flask was charged with nitrogen after three cycles of freeze-pump-thaw. The solution was heated to reflux

for $72 \mathrm{~h}$. The mixture was poured into methanol. The precipitated solid was placed in a Soxhlet thimble, and extracted consecutively with methanol (12 h), acetone (12 h), ethyl acetate (12 h), hexane (24 h), dichloromethane (12 h), and chloroform (12 h), respectively. The chloroform fraction was precipitated in methanol, and then filtered and dried in vacuum to give $34 \mathrm{mg}$ of the final product as a black solid in a 
yield of $56 \%$. GPC at room temperature using THF as eluent: $M_{\mathrm{n}}=45 \mathrm{kDa} ; M_{\mathrm{w}} / M_{\mathrm{n}}$ $=2.5$. (For Stille-B1) and 72\% yield, with GPC at room temperature using THF as eluent: $M_{\mathrm{n}}=30 \mathrm{kDa} ; M_{\mathrm{w}} / M_{\mathrm{n}}=2.2$.

\subsubsection{GPC traces for polymers and NMR spectra}

\subsubsection{GPC traces for polymers}
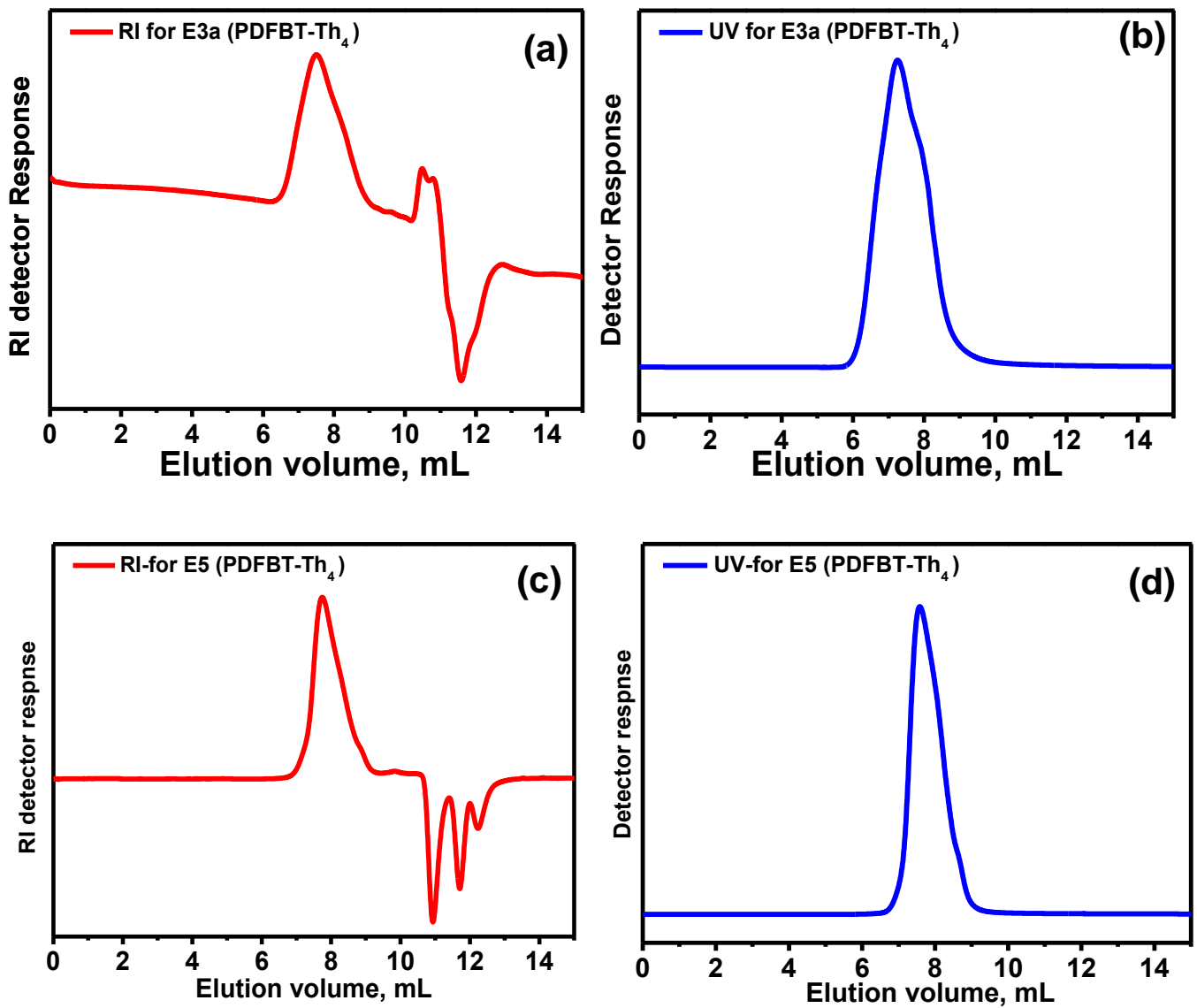

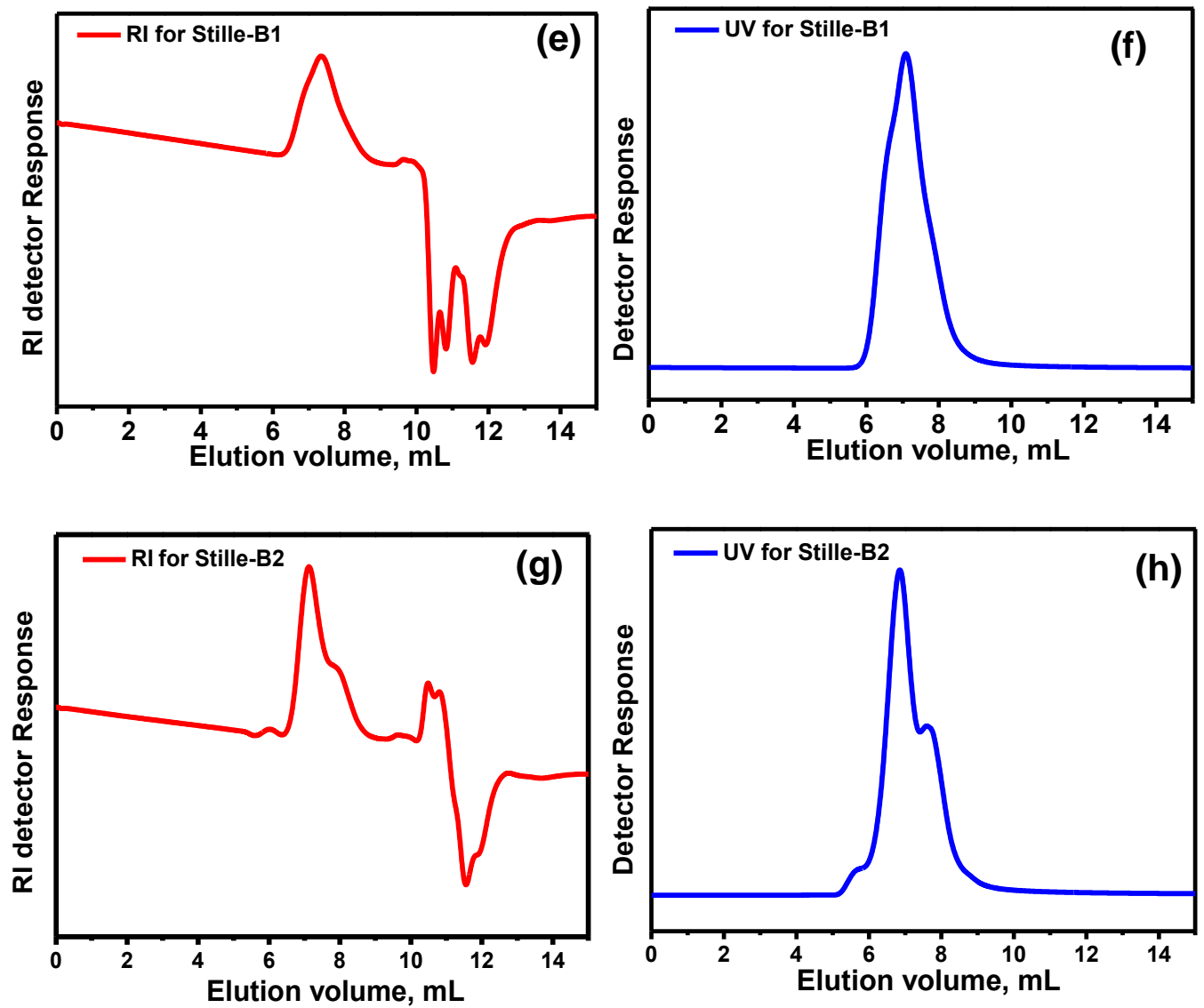

Figure 5.1. GPC traces for PDFBT-Th4 (using THF as eluent at r.t.) from RI (a, c, e, and g) and UV (550 nm) (b, d, f, and h) detectors, respectively. 


\subsubsection{NMR spectra of monomers}

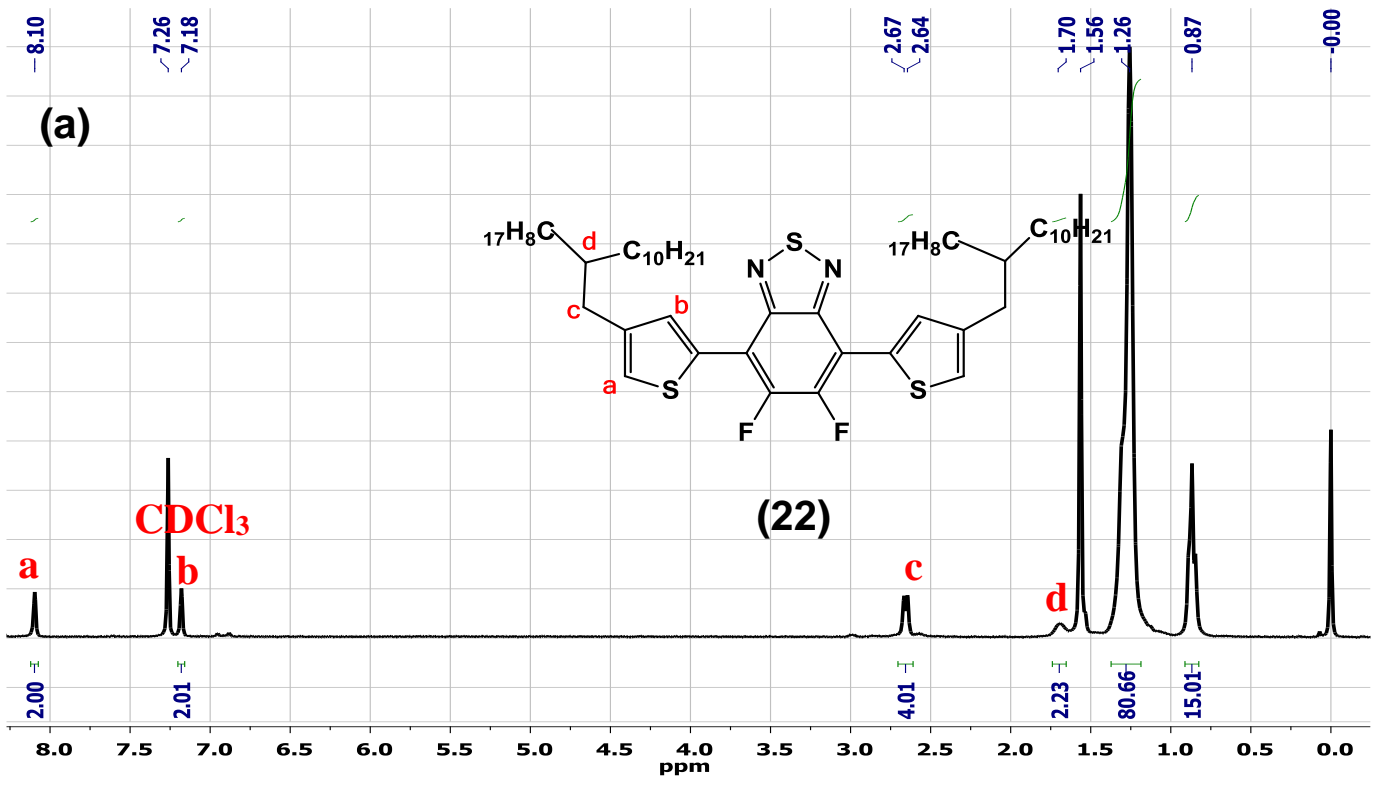

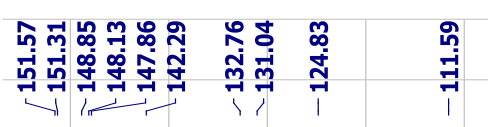

(b)

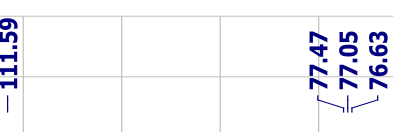

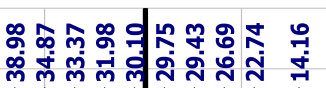

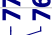
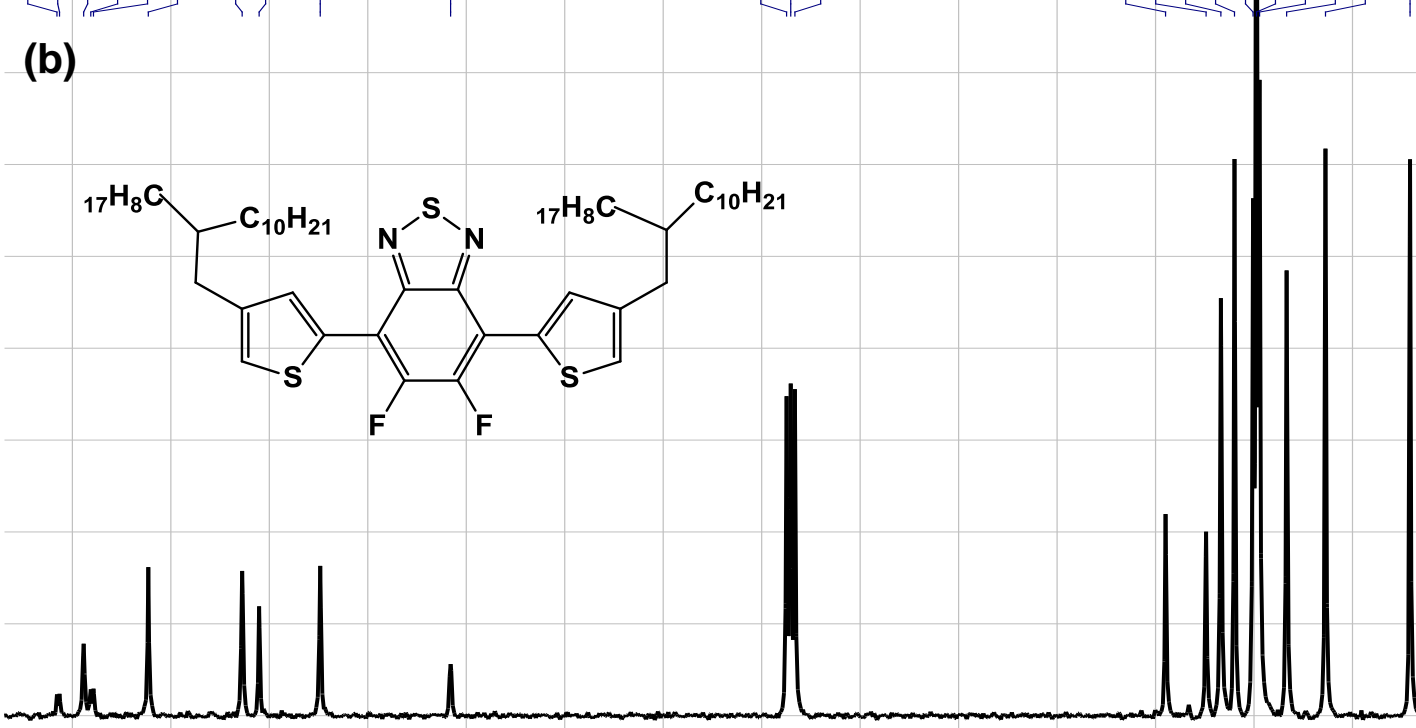

$\begin{array}{lllllll}150 & 140 & 130 & 120 & 110 & 100 & 90\end{array}$

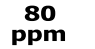




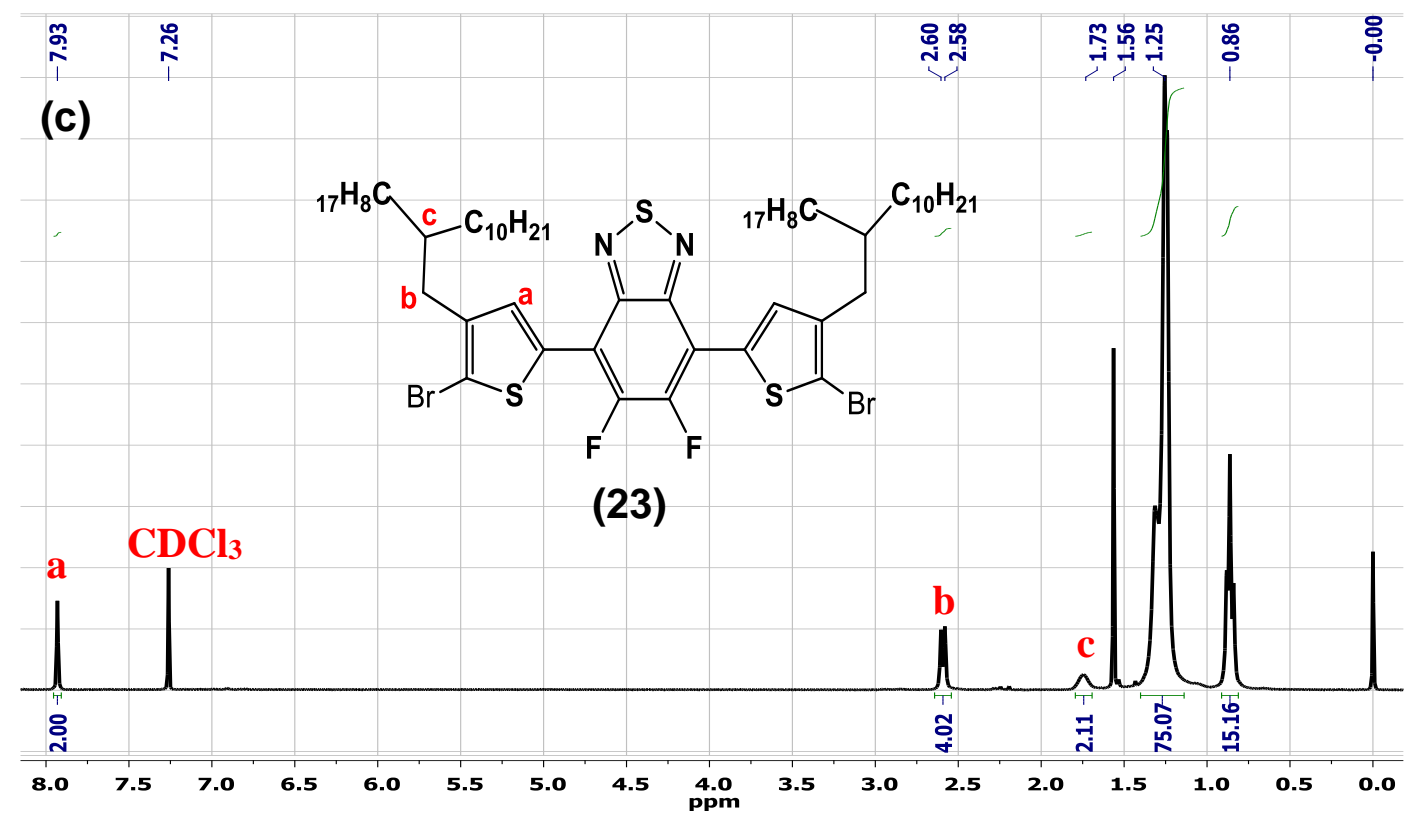

Figure 5.2. ${ }^{1} \mathrm{H}$ NMR (300 MHz) spectra for compound 22 (a) and 23 (c); ${ }^{13} \mathrm{C}$ NMR (300 MHz) spectra for compound 22 (b) in $\mathrm{CDCl}_{3}$ solvent at R.T.

\subsection{Results and Discussion}

\subsubsection{Synthesis and structural characterization of PDFBT-Th4 polymer}

Compound 22 (see Scheme 9) was synthesized via $\operatorname{Pd}_{2}(\mathrm{dba})_{3}$-catalyzed direct arylation coupling between DBrDFBT and thiophene with an attached branched alkyl chain to ensure solubility of the target polymer. Its optimization has been done via changing the feed ratio of alkylthiophene from 20 to 10 equiv. with respect to DBrDFBT monomer, thus the yield of Compound 22 was improved from $55.7 \%$ to $61.0 \%$, which was comparable to that from Stille coupling as reported in the literature. ${ }^{84,296,303}$ The subsequent bromination Compound 22 using NBS gave 
Compound 23 in $89 \%$ yield. The purity of both Compound 22 and 23 was confirmed with ${ }^{1} \mathrm{H}$ - and ${ }^{13} \mathrm{C}$-NMR spectra (see section 5.4.4)

The synthetic route to the PDFBT-Th4 polymer via DAP is shown in Scheme 9, where Compound $\mathbf{2 3}$ and 2,2'-bithiophene were reacted under different DAP reaction conditions aiming to get good quality PDFBT-Th4 polymer with high molecular weight. Therefore, the optimization of DAP reaction was carried out via changing palladium-catalyst, phosphine-ligand, base, solvent, reaction time, and temperature (see Table 5.1). First, Compound 23 and 2,2'-bithiophene were reacted in the presence of $\mathrm{Pd}_{2}(\mathrm{dba})_{3}$ catalyst, (o- $\left.\mathrm{MeOPh}\right)_{3} \mathrm{P}$ ligand, $\mathrm{PivOH}$ additive, $\mathrm{K}_{2} \mathrm{CO}_{3}$ base, and o-xylene solvent $(0.2 \mathrm{M})$ at $100{ }^{\circ} \mathrm{C}$ for $24 \mathrm{~h}$, (Entry E1) which was previously employed for the synthesis of high molecular weight 2,1,3benzothidiazole copolymers. ${ }^{84}$ However, in our system, the only low yield of $11 \%$ $\left(M_{\mathrm{n}}\right.$ of $\left.11 \mathrm{kDa}\right)$ was obtained from chloroform fraction. The effort to further increase the yield of chloroform fraction and molecular weight was carried out by increasing the reaction temperature to $140{ }^{\circ} \mathrm{C}$ (Entry E2), which gives a polymer with slightly increased molecular weight $\left(M_{\mathrm{n}}\right.$ of $\left.13 \mathrm{kDa}\right)$ but its yield was almost similar to that of E1. This implies that the degree of polymerization was not improved with the increase of reaction temperature. The effect of solvent on the reactivity of $\operatorname{Pd}_{2}(\mathrm{dba})_{3}$ catalyst was investigated using DMAc (Entry E7) and chlorobenzene (Entry E8), where no polymer was obtained in DMAc as the reaction mixture did not precipitate in methanol after $24 \mathrm{~h}$ reaction at $100{ }^{\circ} \mathrm{C}$. However, in chlorobenzene, a 
polycondensation product was obtained mainly from hexane fraction $\left(M_{\mathrm{n}}=6.2 \mathrm{kDa}\right)$. Therefore, it could be inferred that less polar solvents might promote the degree of polymerization.

Table 5.1. DAP reaction optimization entries for the synthesis of the PDFBT-Th 4 polymer.

\begin{tabular}{|c|c|c|c|c|c|c|c|}
\hline $\begin{array}{l}\text { Entry } \\
\text { no. }\end{array}$ & Catalyst & Solvent/Base & Ligand & $\begin{array}{l}\text { Time } \\
\text { (h) }\end{array}$ & $\begin{array}{l}\text { Yield } \\
(\%)\end{array}$ & $\begin{array}{l}{ }^{e} M_{\mathrm{n}} \\
(\mathrm{kDa})\end{array}$ & PDI \\
\hline E1 & $\mathrm{Pd}_{2}(\mathrm{dba})_{3}$ & o-xylene $/ \mathrm{K}_{2} \mathrm{CO}_{3}$ & $(\mathrm{o}-\mathrm{MeOPh})_{3} \mathrm{P}$ & 24 & $11^{b}$ & 11 & 2.2 \\
\hline $\mathbf{E 2}^{\mathbf{a}}$ & $\mathrm{Pd}_{2}(\mathrm{dba})_{3}$ & o-xylene $/ \mathrm{K}_{2} \mathrm{CO}_{3}$ & $(\mathrm{o}-\mathrm{MeOPh})_{3} \mathrm{P}$ & 24 & $14^{b}$ & 13 & 3.0 \\
\hline E3c & $\mathrm{Pd}(\mathrm{OAc})_{2}$ & o-xylene $/ \mathrm{K}_{2} \mathrm{CO}_{3}$ & $(\mathrm{o}-\mathrm{MeOPh})_{3} \mathrm{P}$ & 24 & $85^{\mathrm{c}}$ & 11 & 1.9 \\
\hline E3b & $\mathrm{Pd}(\mathrm{OAc})_{2}$ & o-xylene $/ \mathrm{K}_{2} \mathrm{CO}_{3}$ & $(\mathrm{o}-\mathrm{MeOPh})_{3} \mathrm{P}$ & 48 & $83^{c}$ & 10 & 1.7 \\
\hline E3a & $\mathrm{Pd}(\mathrm{OAc})_{2}$ & o-xylene $/ \mathrm{K}_{2} \mathrm{CO}_{3}$ & $(\mathrm{o}-\mathrm{MeOPh})_{3} \mathrm{P}$ & 72 & $81^{b}$ & 17 & 2.8 \\
\hline E4 & $\mathrm{Pd}(\mathrm{OAc})_{2}$ & o-xylene/ $\mathrm{K}_{2} \mathrm{CO}_{3}$ & $\mathrm{PCy}_{3} \cdot \mathrm{HBF}_{4}$ & 24 & $\mathrm{~d}_{-}$ & - & - \\
\hline E5 & $\mathrm{Pd}(\mathrm{OAc})_{2}$ & o-xylene/ $\mathrm{Cs}_{2} \mathrm{CO}_{3}$ & $(\mathrm{o}-\mathrm{MeOPh})_{3} \mathrm{P}$ & 24 & $81^{\mathrm{c}}$ & 15 & 2.0 \\
\hline E6 & Herrmann & o-xylene $/ \mathrm{K}_{2} \mathrm{CO}_{3}$ & $(\mathrm{o}-\mathrm{MeOPh})_{3} \mathrm{P}$ & 24 & $67^{\mathrm{c}}$ & 3 & 2.4 \\
\hline E7 & $\mathrm{Pd}_{2}(\mathrm{dba})_{3}$ & $\mathrm{DMAc} / \mathrm{K}_{2} \mathrm{CO}_{3}$ & $(\mathrm{o}-\mathrm{MeOPh})_{3} \mathrm{P}$ & 24 & $d_{-}$ & - & - \\
\hline E8 & $\mathrm{Pd}_{2}(\mathrm{dba})_{3}$ & $\mathrm{CB} / \mathrm{K}_{2} \mathrm{CO}_{3}$ & $(\mathrm{o}-\mathrm{MeOPh})_{3} \mathrm{P}$ & 24 & $79^{c}$ & 6.2 & 1.7 \\
\hline Stille-B1 & $\mathrm{Pd}_{2}(\mathrm{dba})_{3}$ & o-xylene & $\mathrm{P}(\text { o-tolyl })_{3}$ & 72 & $56^{b}$ & 28 & 2.5 \\
\hline Stille-B2 & $\mathrm{Pd}_{2}(\mathrm{dba})_{3}$ & o-xylene & $\mathrm{P}(\text { o-tolyl })_{3}$ & 72 & $71^{b}$ & 30 & 2.2 \\
\hline
\end{tabular}

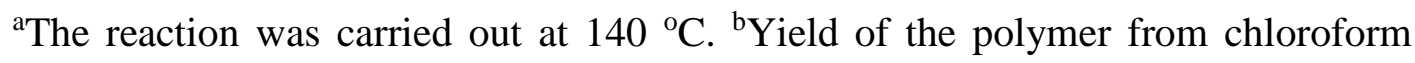
fraction. ${ }^{c}$ Yield of the polymer from hexane fraction and no polymer in chloroform fraction. ${ }^{\mathrm{d}}$ No precipitate in methanol. ${ }^{\mathrm{e}}$ Measured by GPC with polystyrene as 
standard and THF as eluent at room temperature. For all entries; PiVOH additive, monomer concentration, $0.2 \mathrm{M}$.

In order to investigate the effect of a catalyst on DAP reactions between Compound $\mathbf{2 3}$ and 2,2'-bithiophene, Herrmann's catalyst (Entry E6) was employed to give a polycondensation product mainly from hexane fraction (mainly oligomers) with $M_{\mathrm{n}}$ of $3 \mathrm{kDa}$. Therefore, from E1, E2, E6, and E8, it could be inferred that palladium catalyst with oxidation sate of $\mathrm{Pd}(0)$ is not reactive enough to give high molecular weight PDFBT-Th4 polymer. From literature, we have learned that $\mathrm{Pd}(\mathrm{OAc})_{2}$ has been used in the DAP synthesis of DFBT based small molecules and high molecular weight copolymers. ${ }^{293-295}$ Therefore, $\mathrm{Pd}(\mathrm{OAc})_{2}$ has been employed in the DAP reactions of Compound $\mathbf{2 3}$ with 2,2'-bithiophene in the presence of (o$\mathrm{MeOPh})_{3} \mathrm{P}$ ligand, $\mathrm{PivOH}$ additive, $\mathrm{K}_{2} \mathrm{CO}_{3}$ base and o-xylene $(0.2 \mathrm{M})$ at $100{ }^{\circ} \mathrm{C}$ for $24 \mathrm{~h}$ (Entry E3c) to give majority of low molecular weight polymer $\left(M_{\mathrm{n}}=11 \mathrm{kDa}\right)$ collected from hexane fraction. Upon increasing the reaction time to $48 \mathrm{~h}$ there was no significant improvement in reaction yield and molecular weight. However, as the reaction time reached to $72 \mathrm{~h}$ (Entry E3a), the color of the reaction mixture changed from dark-red to dark-purple. After precipitation in methanol and Soxhlet extraction, PDFBT-Th4 polymer with $M_{\mathrm{n}}$ as high as $17 \mathrm{kDa}$ was isolated in $81 \%$ yield from chloroform fraction. This implies that $\mathrm{Pd}(\mathrm{OAc})_{2}$, where $\mathrm{Pd}$ in $(+2)$ oxidation state 
combined with an extended reaction time results in the DAP reaction of Compound 23 with 2,2'-bithiophene towards a high degree of polymerization.

The effect of ligand and base on $\mathrm{Pd}(\mathrm{OAc})_{2}$ catalyzed DAP reaction was studied by using $\mathrm{PCy}_{3} . \mathrm{HBF}_{4}($ Entry $\mathbf{E 4})$ and $\mathrm{Cs}_{2} \mathrm{CO}_{3}$ strong base (Entry E5) for 24 h. In $\mathbf{E 4}$ no polycondensation product was obtained, which implies (o-MeOPh) ${ }_{3} \mathrm{P}$ is a better ligand, whereas using a stronger base $\mathrm{Cs}_{2} \mathrm{CO}_{3}$ led to relatively high molecular weight of PDFBT-Th4 $\left(M_{\mathrm{n}}=15 \mathrm{kDa}, 81 \%\right.$ yield $)$ from hexane fraction as compared to $\mathrm{K}_{2} \mathrm{CO}_{3}$ of E3c. Based on the aforementioned results, $\mathrm{Pd}(\mathrm{OAc})_{2} /(\mathrm{o}-$ $\mathrm{MeOPh})_{3} \mathrm{P} / \mathrm{PivOH} / \mathrm{K}_{2} \mathrm{CO}_{3} / \mathrm{o}-\mathrm{xylene}$ of $\mathbf{E 3 a}$ is the best reaction condition to obtain high molecular weight and yield of PDFBT-Th4 via DAP.

For comparison, PDFBT-Th4 polymer was synthesized in two batches via Stille polycondensation of 4,7-dibromo-5,6-difluorobenzo[c][1,2,5]thiadiazole (Compound 23) and 5,5'-bis(trimethylstannyl)-2,2'-bithiophene in the same type of solvent (o-xylene) with DAP (see Scheme 9). PDFBT-Th4 polymer (labeled as Stille-B1) with $M_{\mathrm{n}}=28 \mathrm{kDa}$ and PDI $=2.5$ were obtained in a yield of $56 \%$, and also the second batch (Stille-B2) was synthesized in $72 \%$ yield with $M_{\mathrm{n}}=30 \mathrm{kDa}$ and PDI=2.2. This result indicates that $M_{\mathrm{n}}$ of PDFBT-Th4 polymer obtained by DAP is still lower than that of Stille polymerization method. However, this work indicates that it could be possible to synthesize PDFBT-Th4 polymer via DAP and an extensive DAP reaction optimization could lead to further increase in its molecular weight. For instance Leclerc et al. have reported high molecular weight DAP 
polymer using another $\mathrm{Pd}(+2)$ catalyst (like $\left.\mathrm{Pd}\left(\mathrm{PPh}_{3}\right)_{2} \mathrm{Cl}_{2}\right)$, which shows good selectivity. Another optimization could be done by using $\mathrm{Pd}(\mathrm{OAc})_{2}$ catalyst via a changing monomer concentration with/without phosphine ligand might lead to the synthesis of high molecular weight PDFBT-Th4 polymer. $^{304-306}$

The chemical structures of selected PDFBT-Th4 polymers synthesized via DAP (E3a) were characterized by using high temperature $\left(100{ }^{\circ} \mathrm{C}\right){ }^{1} \mathrm{H}-\mathrm{NMR}$ spectra collected in $\mathrm{C}_{2} \mathrm{D}_{2} \mathrm{Cl}_{4}$ solvent and then compared with the spectra of PDFBT-Th4 polymers synthesized by Stille polymerization method in two batches (Stille-B1 and -B2), which shows similar polymer structures (see Figure 5.3). No evidence of branching can be observed, and the extra little peaks found in the ${ }^{1} \mathrm{H}-\mathrm{NMR}$ spectra could be related to different end groups. The selectivity of $(\alpha) \mathrm{C}-\mathrm{H}$ over $(\beta) \mathrm{C}-\mathrm{H}$ bond on 2,2'-bithiophene is good as it is already described by Leclerc and coworkers i.e., bromination of 2,2'-bithiophene is at $(\alpha) \mathrm{C}-\mathrm{H}$ potions could induce the branching at $(\beta) \mathrm{C}-\mathrm{H} \cdot{ }^{304,305}$ Therefore, we have used non-brominated 2,2'-bithiophene in the DAP synthesis of PDFBT-Th4 polymers. 




Figure 5.3. ${ }^{1} \mathrm{H}$ NMR spectra of the PDFBT-Th4 polymers synthesized via DAP, E3a (a), Stille-B1 (b), and Stille-B2 (c) at $100{ }^{\circ} \mathrm{C}$ in $\mathrm{C}_{2} \mathrm{D}_{2} \mathrm{Cl}_{4}$.

\subsubsection{Thermal Properties of PDFBT-Th 4 Polymer}

The thermal stability of PDFBT-Th4 polymers was investigated using thermogravimetric analysis (TGA) with a heating rate of $10{ }^{\circ} \mathrm{C} \min ^{-1}$ under $\mathrm{N}_{2}$ atmosphere. The decomposition temperature ( $T_{\mathrm{d}}$, corresponding to a $5 \%$ weight loss) is located at $394{ }^{\circ} \mathrm{C}$ for both PDFBT-Th4 polymers obtained by DAP (E3a) and 
Stille coupling (see Figure 5.4), demonstrating their sufficient high thermal stability for the application in organic electronic devices.

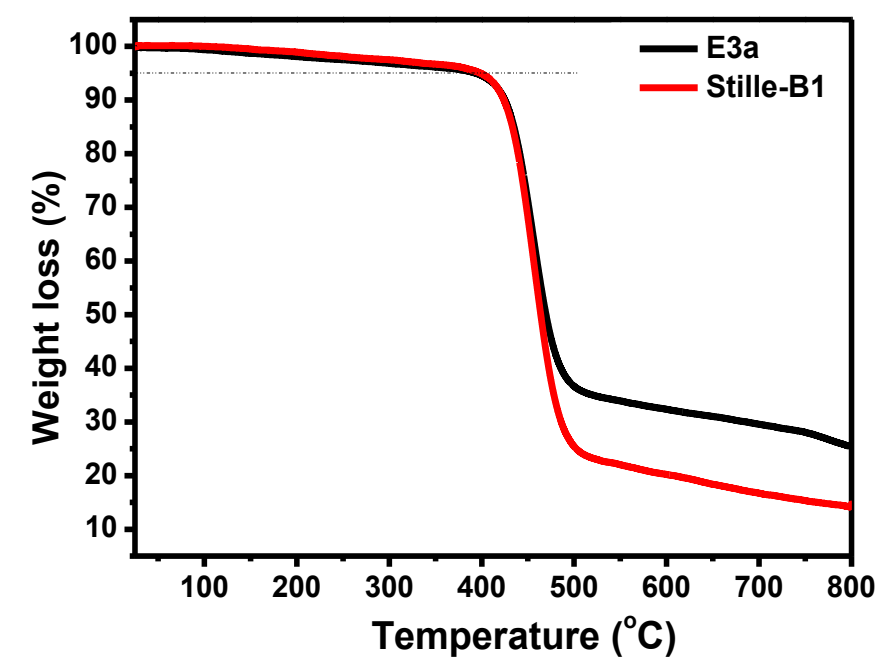

Figure 5.4. TGA plots of PDFBT-Th4 polymers with a heating rate of $10{ }^{\circ} \mathrm{C} \mathrm{min}{ }^{-1}$ under a nitrogen atmosphere.

\subsubsection{Optical Properties of PDFBT-Th 4 polymer}

The UV-vis absorption spectra for selected PDFBT-Th4 polymers were acquired from both chloroform and chlorobenzene solutions and spin-cast thin films. In addition to this, the polymer aggregation and de-aggregation behaviors were investigated by using temperature dependent UV-vis spectroscopy. The spectra are shown in Figure 5.5 (a-j) and the relevant data are summarized in Table 5.2. Broad absorption bands across the visible region were observed for both solution and thin films spectra. The absorption maxima for E3a and Stille-B1 polymers in chlorobenzene solution are located at 553 and $566 \mathrm{~nm}$, respectively and their 
chloroform spectra are red shifted by ca. 18 and $22 \mathrm{~nm}$, respectively that could be due to strong aggregation ability of the polymers in chloroform solution. However, StilleB2 polymer shows a strong aggregation with the appearance of a 0-0 vibrational peak at $693 \mathrm{~nm}$ as compared with the other two polymers, so that its absorbance is red shifted (see Figure 5.5 (b)).

The aggregation and de-aggregation properties of the polymers were examined by collecting UV-vis spectra of polymers upon heating and cooling of their chlorobenzene solution. Upon heating from 25 to $70{ }^{\circ} \mathrm{C}$ (see Figure 5.5 (c, e, \& g)), the absorption maxima for both E3a and Stille (B1 \& B2) polymers were blue-shifted due to the de-aggregation of polymers chains which might result in twisting (or decreased in co-planarity) of the polymer backbone and thus decrease in conjugation length of PDFBT-Th4 polymer. However, upon cooling reversible red-shifted absorption spectra were observed due to the formation of aggregates (see Figure 5.5 $(\mathrm{d}, \mathrm{f}, \& \mathrm{~h})) .{ }^{129,296}$ The thin-film of PDFBT-Th4 polymers exhibit $\lambda_{\max }$ red shifted (ca.127 nm for E3a and ca.114 $\mathrm{nm}$ for Stille-B1 polymers) as compared with their chlorobenzene solution absorption spectra. However, Stille-B2 polymer exhibit a slightly blue shift (ca. $5 \mathrm{~nm}$ ) as compared with its solution spectra. The thin-film spectra of the polymers exhibit three well-resolved vibrational peaks at $680 \mathrm{~nm}(0-$ 0), $625 \mathrm{~nm}(0-1)$, and $447 \mathrm{~nm}(0-2)$ for all PDFBT-Th4 polymers with slightly red shifted and high intensity of 0-0 peak for Stille-B2. Which is an indication for the presence of strong J-aggregation and thus intermolecular interaction in solid thin 
films. Upon annealing, at $100{ }^{\circ} \mathrm{C}$ no significant changes were observed except an enhanced intensity of 0-0 peak for E3a polymer. The corresponding optical bandgap, $\mathrm{E}_{\mathrm{g}}{ }^{\text {opt }}$ was estimated from $\lambda_{\text {onset }}$ of the r.t. thin film absorption spectra is $1.66 \mathrm{eV}$ (for both E3a, Stille-B1) and $1.65 \mathrm{eV}$ (for Stille-B2) polymers (see Table 5.2).
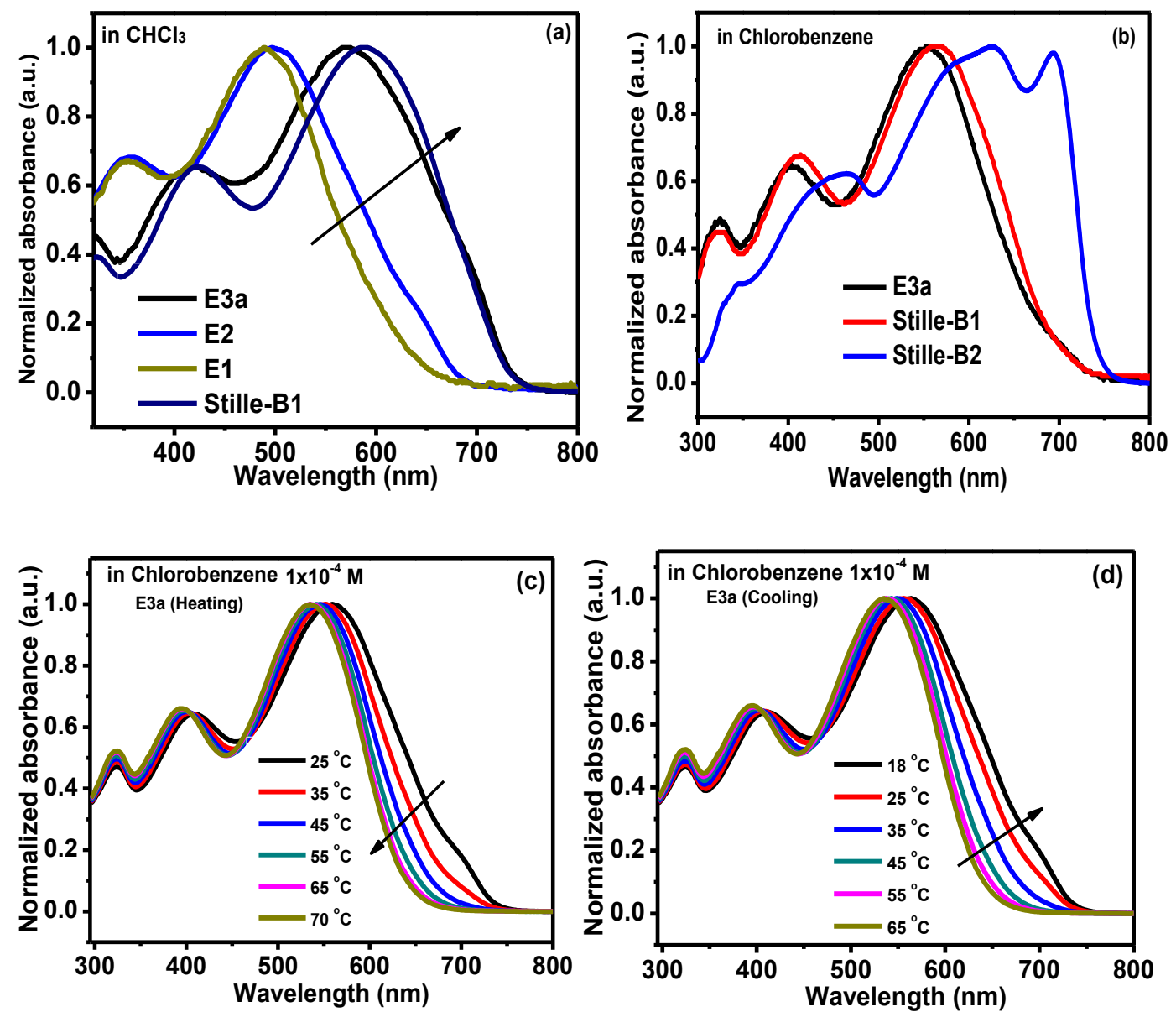



Figure 5.5. UV-vis absorption spectra from $\mathrm{CHCl}_{3}$ solution (a) and from $\mathrm{CB}$ solution

(b). Temperature dependent spectra for E3a, Stille-B1, and Stille-B2 polymers upon 
heating (c, e, \& g) and upon cooling (d, f, \& h), respectively. Thin film spectra for E3a, Stille-B1, and Stille-B2 polymers at r.t (i) and after annealing at $100{ }^{\circ} \mathrm{C}$ (j) of PDFBT-Th4 polymers.

Table 5.2. Optical data of PDFBT-Th4 polymers

\begin{tabular}{|c|c|c|c|c|c|c|}
\hline \multirow{2}{*}{$\begin{array}{l}\text { PDFBT- } \\
\text { Th4 } \\
\text { Polymers }\end{array}$} & \multicolumn{2}{|c|}{ In Chlorobenzene } & \multicolumn{3}{|c|}{ Film } & \multirow[b]{2}{*}{$\begin{array}{l}{ }^{e} E_{g}{ }^{o p t} \\
(e V)\end{array}$} \\
\hline & $\begin{array}{l}\lambda_{\max } \\
{[n m]}\end{array}$ & $\begin{array}{l}\lambda_{\text {onset }} \\
\text { [nm] }\end{array}$ & $\begin{array}{l}c^{c} \lambda_{\max } \\
{[\mathrm{nm}]}\end{array}$ & $\begin{array}{l}{ }^{d} \lambda_{\max } \\
{[n \operatorname{mm}]}\end{array}$ & $\begin{array}{l}\lambda_{\text {onset }} \\
{[n m]}\end{array}$ & \\
\hline E3a & $571^{a} / 553^{b}$ & $737^{\mathbf{a}} / 736^{\mathrm{b}}$ & $\begin{array}{l}680^{0-0} / 625^{0-1} / \\
447^{0-2}\end{array}$ & $\begin{array}{l}680^{0-0} / 625^{0-1} / \\
446^{0-2}\end{array}$ & $\begin{array}{l}746^{c /} \\
752^{d}\end{array}$ & 1.66 \\
\hline Stille-B1 & $588^{a} / 566^{b}$ & $737^{\mathbf{a}} / 736^{\mathbf{b}}$ & $\begin{array}{l}680^{0-0} / 625^{0-1} / \\
447^{0-2}\end{array}$ & $\begin{array}{l}680^{0-0} / 625^{0-1} / \\
446^{0-2}\end{array}$ & $\begin{array}{l}746^{c /} \\
749^{d}\end{array}$ & 1.66 \\
\hline Stille-B2 & $\begin{array}{l}\text { b } 693^{0-0} / 626^{0-1} / \\
459^{0-2}\end{array}$ & $749^{b}$ & $\begin{array}{l}688^{0-0} / 626^{0-1} / \\
450^{0-2}\end{array}$ & $\begin{array}{l}688^{0-0} / 628^{0-1} / \\
450^{0-2}\end{array}$ & $\begin{array}{l}752^{c /} \\
752^{d}\end{array}$ & 1.65 \\
\hline
\end{tabular}

${ }^{\mathrm{a} I n} \mathrm{CHCl}_{3},{ }^{\mathrm{b}}$ In Chlorobenzene (CB), ${ }^{\mathrm{c}}$ Film at room temperature. ${ }^{\mathrm{d}}$ Film at $100{ }^{\circ} \mathrm{C}$ annealing temperature. ${ }^{\mathrm{e}} \mathrm{E}_{\mathrm{g}}{ }^{\mathrm{opt}}(\mathrm{eV})=1240 /{ }^{c} \lambda_{\text {onset }}[\mathrm{nm}]$.

\subsubsection{Electrochemical properties of PDFBT-Th 4 polymer}

The electrochemical properties of PDFBT-Th4 polymers were investigated by cyclic voltammetry (CV) analysis using ferrocene standard (see Figure 5.6). Table 5.3, summarizes relevant data for both E3a and Stille polymers. The $\mathrm{HOMO}^{\text {ele}} / \mathrm{LUMO}^{\text {ele }}$ energy levels were calculated by the following equation: 
$\mathrm{HOMO}^{\text {ele }} / \mathrm{LUMO}^{\text {ele }}=-\left(4.8-\mathrm{E}_{1 / 2}, \mathrm{Fc} / \mathrm{Fc}^{+}+\mathrm{E}_{\text {ox }} / \mathrm{E}_{\text {red }}\right) \mathrm{eV}$, where $\mathrm{E}_{1 / 2}, \mathrm{Fc} / \mathrm{Fc}^{+}=0.46 \mathrm{~V}$ (for E3a and Stille-B1) and $\mathrm{E}_{1 / 2}, \mathrm{Fc} / \mathrm{Fc}^{+}=0.6 \mathrm{~V}$ (for Stille-B2). Therefore, $\mathrm{HOMO}^{\text {ele }} / \mathrm{LUMO}^{\text {ele }}$ of -5.45/-3.62, -5.50/-3.61, and -5.43/-3.37 eV for E3a, StilleB1, and Stille-B2 polymers, respectively, from their corresponding onset oxidation $\left(E_{o x}\right)$ and onset reduction $\left(E_{\text {red }}\right)$ potentials. The LUMO ${ }^{\text {opt }}$ calculated by using $\mathrm{LUMO}^{\text {opt }}=\mathrm{HOMO}^{\text {ele }}-\mathrm{Eg}_{\mathrm{g}}{ }^{\text {opt }}$ (see Table 5.3), which is almost similar to the values reported in the literature for the same polymer. ${ }^{296}$ The difference between the bandgap $\left(\mathrm{Eg}^{\text {opt }}\right.$ and $\left.\mathrm{Eg}^{\text {ele }}\right)$ could be due to experimental error.

Table 5.3. Electrochemical properties of PDFBT-Th4 polymers.

\begin{tabular}{|c|c|c|c|c|c|c|}
\hline $\begin{array}{l}\text { PDFBT- } \\
\text { Th}_{4}\end{array}$ & $\begin{array}{l}E_{o x} \\
(V)\end{array}$ & $\begin{array}{l}E_{r e d} \\
(V)\end{array}$ & $\begin{array}{l}{ }^{a} \mathrm{HOMO}^{\text {ele }} \\
(\mathrm{eV})\end{array}$ & $\begin{array}{l}{ }^{a} \mathbf{L U M O} \boldsymbol{O}^{\text {ele }} \\
(\mathrm{eV})\end{array}$ & $\begin{array}{l}{ }^{b} \boldsymbol{L} \boldsymbol{U} M \boldsymbol{O}^{o p t} \\
(\boldsymbol{e} V)\end{array}$ & $\begin{array}{l}{ }^{c} \boldsymbol{E}_{g} \text { ele } \\
(\boldsymbol{e} V)\end{array}$ \\
\hline E3a & 1.10 & -0.72 & -5.45 & -3.62 & -3.80 & 1.83 \\
\hline Stille-B1 & 1.16 & -0.73 & -5.50 & -3.61 & -3.85 & 1.89 \\
\hline Stille-B2 & 1.23 & -0.83 & -5.43 & -3.37 & -3.78 & 2.06 \\
\hline
\end{tabular}



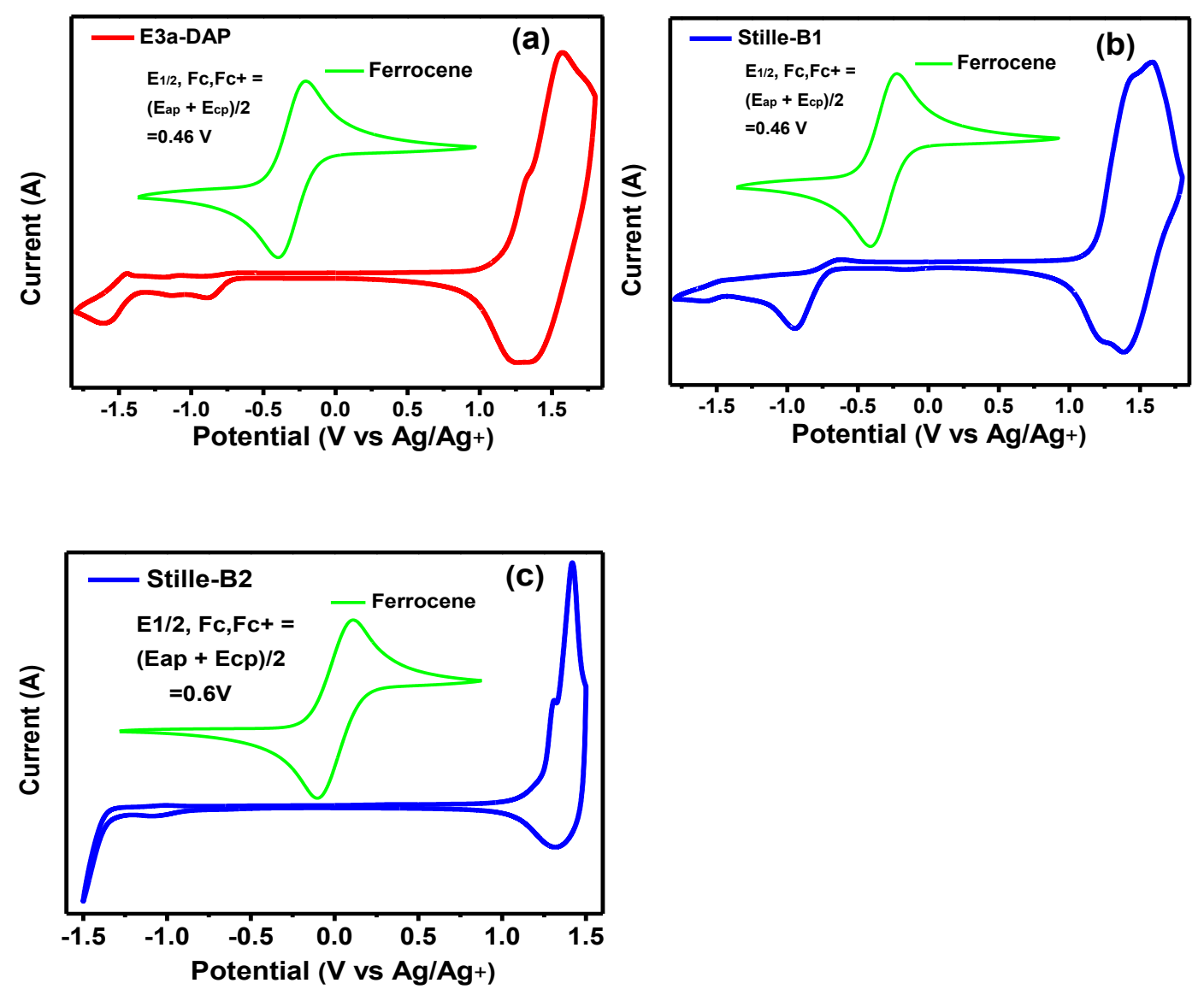

Figure 5.6. Cyclic voltammetry curve for PDFBT-Th4 polymers (a) for E3a and (b) for Stille-B1 (c) for Stille-B2 (inset CV is for ferrocene standard).

\subsubsection{Field-effect transistor properties of PDFBT-Th4 polymer}

The charge-transport properties of PDFBT-Th4 polymers were studied by fabricating bottom-gate, top-contact OTFT devices on OTS-18 modified $\mathrm{Si} / \mathrm{SiO}_{2}$ substrate under ambient conditions. The device performance for selected polymers (E3a, E5, and Stille-B1) are summarized in Table 5.4 and their transfer and output 
characteristics curves are shown in Figure 5.7. The hole mobilities $\left(\mu_{\mathrm{h}}\right)$ derived from the slope of their transfer curve is $2 \times 10^{-3}, 1.3 \times 10^{-3}$, and $3.4 \times 10^{-3} \mathrm{~cm}^{2} \mathrm{~V}^{-1} \mathrm{~s}^{-1}$ for E3a, E5, and Stille-B1 polymers, respectively. This result is consistent with an increase in molecular weight. After thermal annealing at $100{ }^{\circ} \mathrm{C}$, the two DAP polymers (E3a and E5) both synthesized via DAP have displayed an increased $\mu_{\mathrm{h}}$ to $7.5 \times 10^{-3}$ and $2.6 \times 10^{-3} \mathrm{~cm}^{2} \mathrm{~V}^{-1} \mathrm{~s}^{-1}$, respectively, whereas a decreased hole mobility of $2.5 \times 10^{-3} \mathrm{~cm}^{2} \mathrm{~V}^{-1} \mathrm{~s}^{-1}$ was obtained for the Stille-B1 polymer. This could be explained based on weaker intermolecular interaction in the Stille-B1 polymer as it can be seen from its UV-vis spectra, where 0-0 vibrational peak for DAP polymer (E3a) has enhanced in its intensity as compared with the Stille-B1 polymer. These observations are opposite with morphological studies by AFM (see Figure 5.8). At room temperature, both DAP and Stille synthesized polymers (E3a and Stille-B1) have displayed an ordered bicontinuous networks. However, after annealing at 100 ${ }^{\circ} \mathrm{C}$, this phenomenon has greatly enhanced especially for the Stille-B1 polymer, thus we expected the mobility could also increase. Therefore, this decrease in mobility might be due to stannyl-end groups, which could cause charge trapping. ${ }^{307}$ 
Table 5.4. Performance characteristics of OFET devices for PDFBT-Th4 polymers (E3a, E5, and Stille-B1 polymer) semiconductor on an OTS-18-treatd substrate fabricated and measured ambient conditions.

\begin{tabular}{|c|c|c|c|c|c|}
\hline $\begin{array}{l}\text { Entry } \\
\text { no. }\end{array}$ & $\begin{array}{l}M_{n} \\
(\mathbf{k D a})\end{array}$ & $\begin{array}{l}\text { Annealing } \\
\text { Temp. }\left({ }^{\circ} \mathrm{C}\right)\end{array}$ & $\begin{array}{l}\mu_{h} \\
\left(\mathrm{~cm}^{2} \mathrm{~V}^{-1} \mathbf{s}^{-1}\right)\end{array}$ & $\begin{array}{l}V_{t h} \\
\text { (V) }\end{array}$ & $\begin{array}{l}\text { Ion/off } \\
\text { Ratio }\end{array}$ \\
\hline \multirow[t]{2}{*}{ E3a } & \multirow[t]{2}{*}{17} & R.T. & $2.0 \times 10^{-3}$ & 14 & $10^{4}$ \\
\hline & & 100 & $7.5 \times 10^{-3}$ & 16 & $10^{3}-10^{4}$ \\
\hline \multirow[t]{2}{*}{ E5 } & \multirow[t]{2}{*}{15} & R.T. & $1.3 \times 10^{-3}$ & 13 & $10^{3}$ \\
\hline & & 100 & $2.6 \times 10^{-3}$ & 18 & $10^{3}$ \\
\hline \multirow[t]{2}{*}{ Stille- } & \multirow[t]{2}{*}{28} & R.T. & $3.4 \times 10^{-3}$ & 10 & $10^{3}-10^{4}$ \\
\hline & & 100 & $2.5 \times 10^{-3}$ & 10 & $10^{4}$ \\
\hline
\end{tabular}

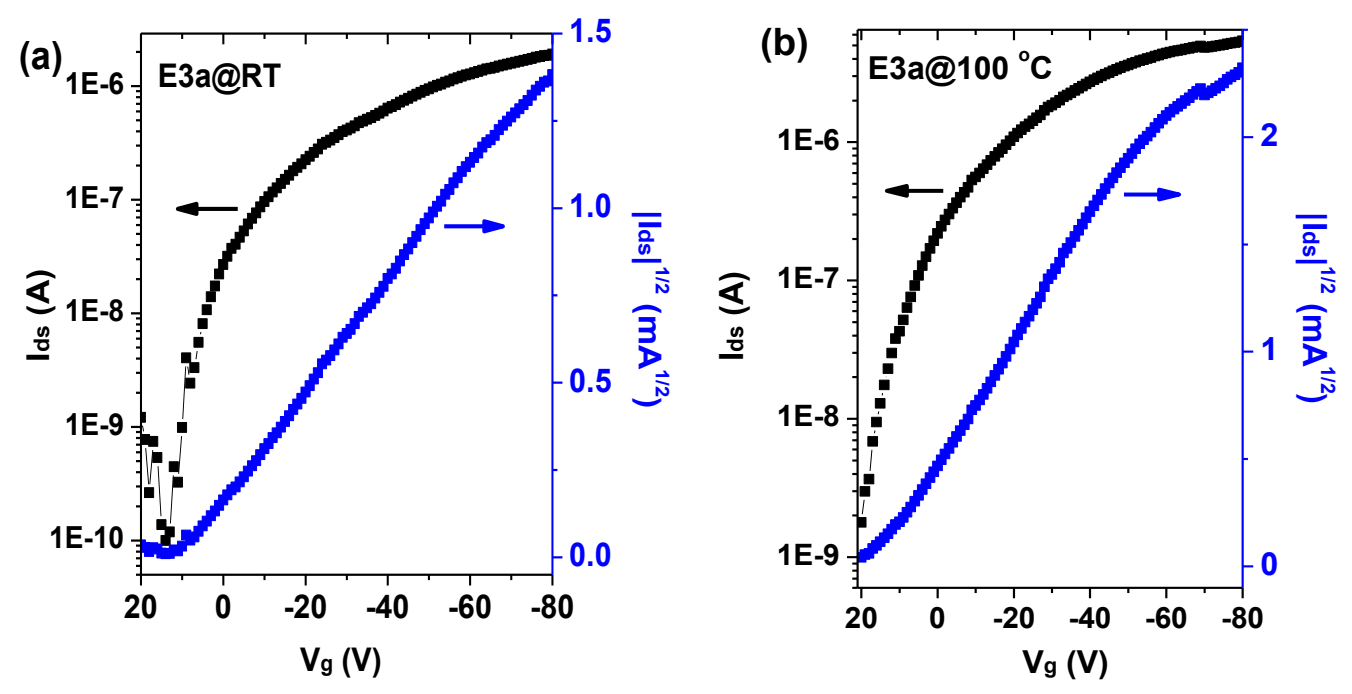



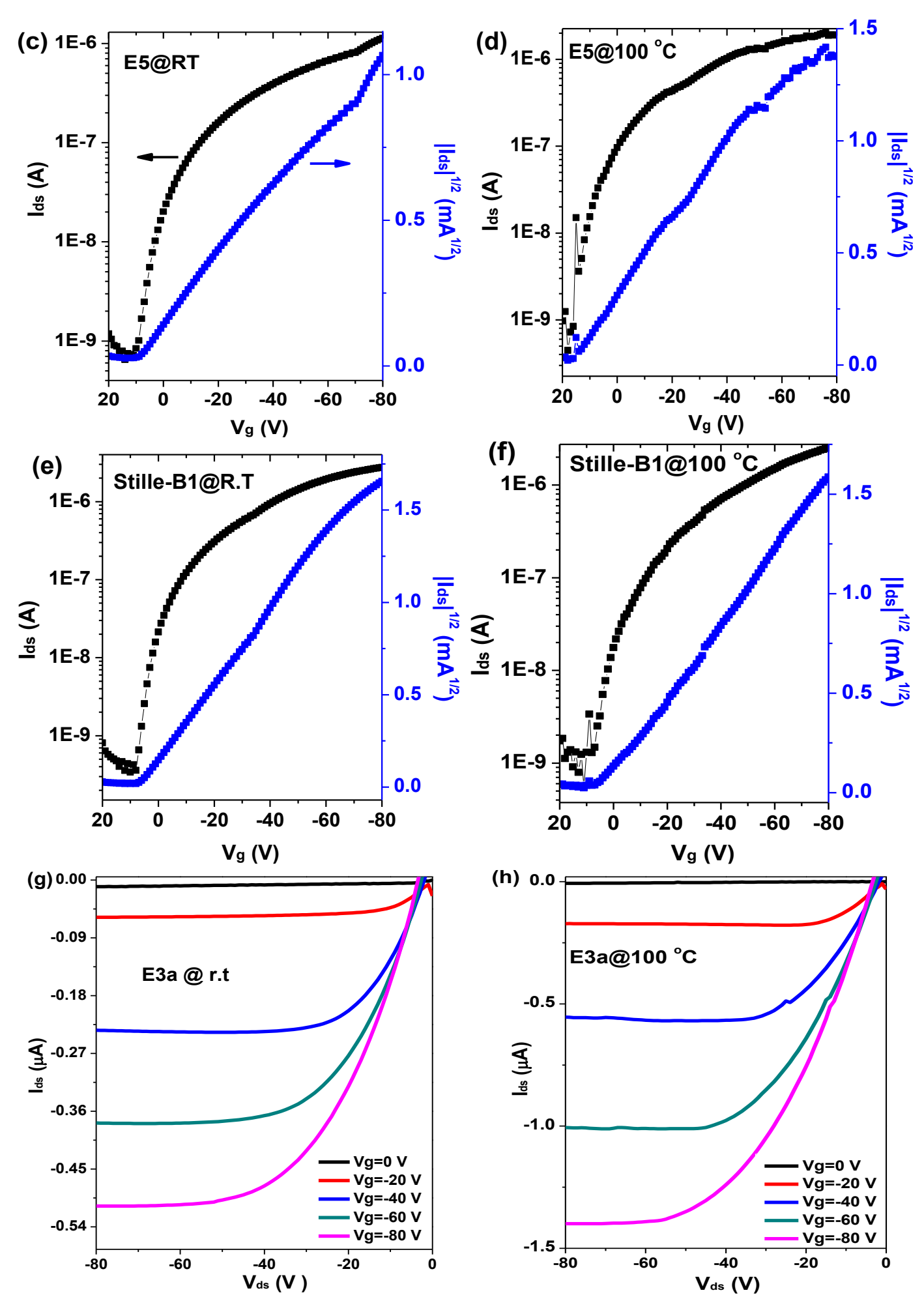

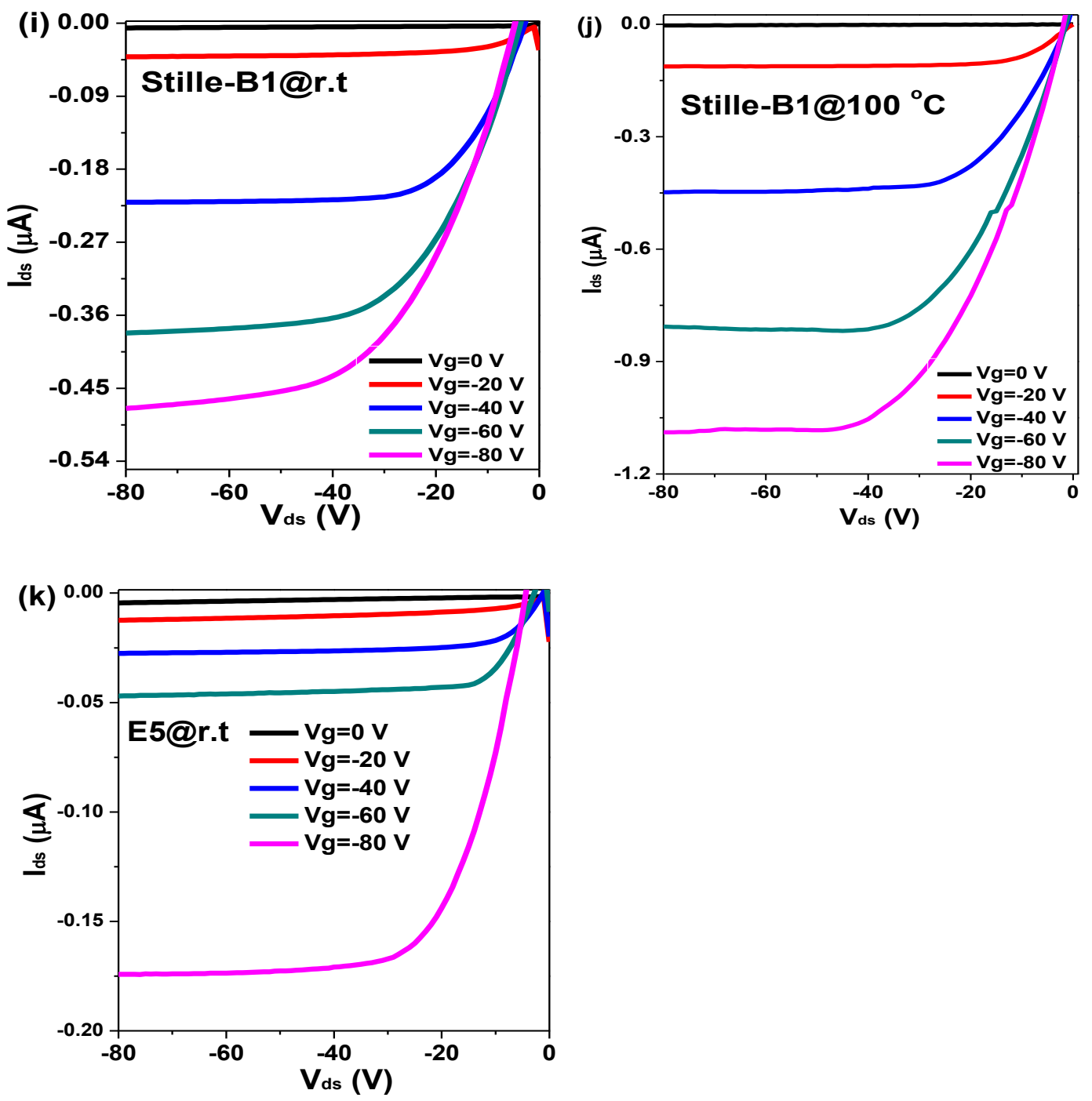

Figure 5.7. Typical transfer curves (a to f) and output characteristics curves ( $\mathrm{g}$ to $\mathrm{k}$ ) for PDFBT-Th4 polymers. 

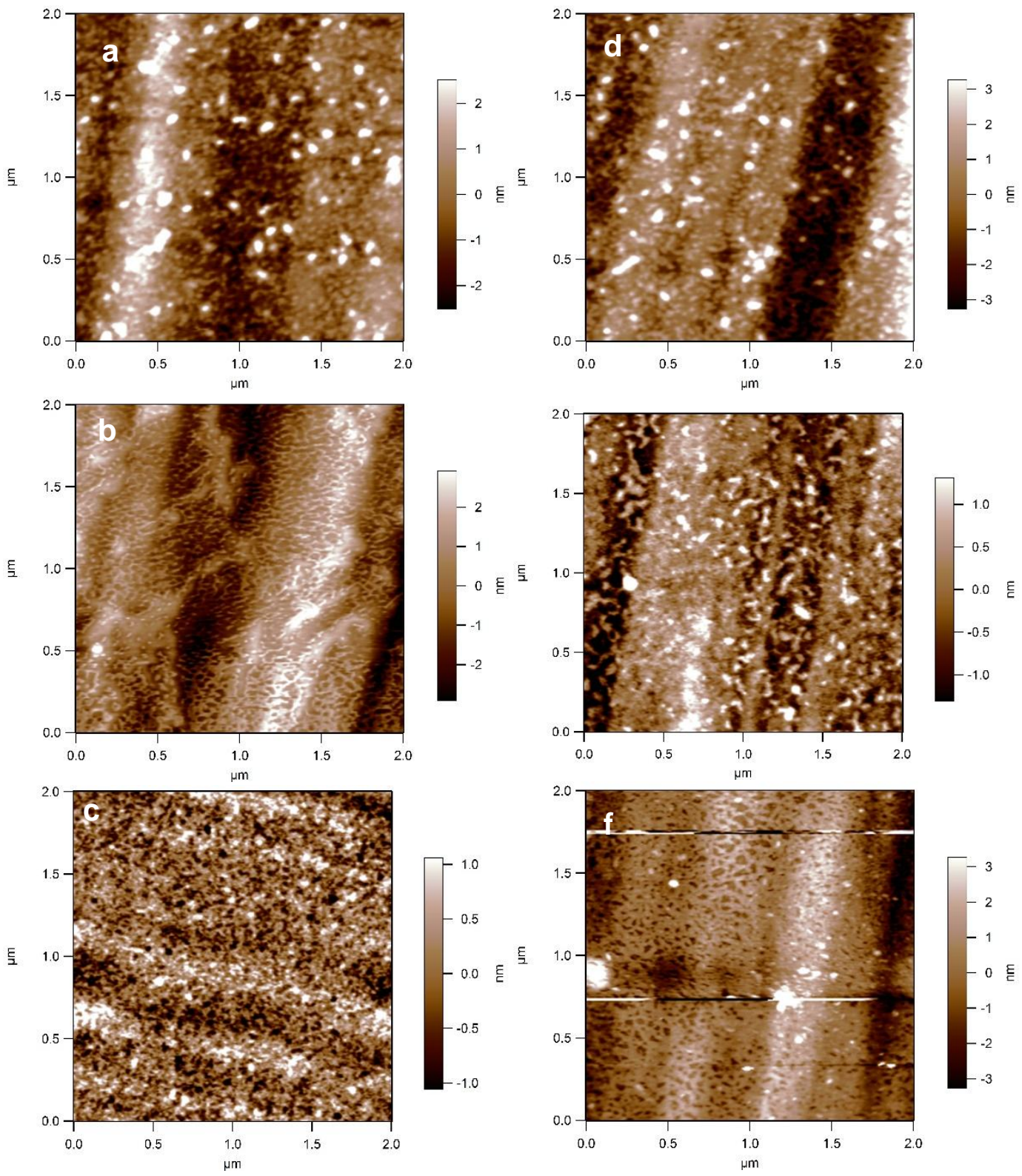

Figure 5.8. AFM height $\left(2 \times 2 \mu \mathrm{m}^{2}\right)$ images for PFFBT-Th4 polymers E3a, E5 and Stille-B1 polymers (a, b, c: at R.T) and (d, e, f: after annealing at $100{ }^{\circ} \mathrm{C}$ ), respectively. 


\subsubsection{Photovoltaic Properties PFFBT-Th 4 Polymers}

Figure 5.9 shows J-V curves of PDFBT-Th4 4 (E3a-DAP and Stille-B2) polymers under illumination of AM $1.5 \mathrm{G}, 100 \mathrm{mWcm}^{-2}$ in an inverted $\mathrm{BHJ}-\mathrm{OPV}$ architecture (indium tin oxide (ITO)/zinc oxide (ZnO)/polymer (PDFBT-Th4):C $\mathrm{C}_{71^{-}}$ butyric acid methyl ester $\left(\mathrm{PC}_{71} \mathrm{BM}\right)(1: 1.2) /$ molybdenum trioxide $\left(\mathrm{MoO}_{3}\right) /$ silver $(\mathrm{Ag}))$. The DAP synthesized polymer of Entry E3a and Stille-B2 polymers of PDFBT-Th4 blended with $\mathrm{PC}_{71} \mathrm{BM}$ (1:1.2 ratio) exhibit a power conversion efficiency (PCE) of $4.12 \%$ and $3.09 \%$, with open circuit voltage $\left(\mathrm{V}_{\mathrm{oc}}\right)$ of 0.77 and $0.52 \mathrm{~V}$, a short-circuit current $\left(\mathrm{J}_{\mathrm{sc}}\right)$ of 12.39 and $12.23 \mathrm{~mA} \mathrm{~cm}^{-2}$, and a fill factor (FF) of $45.7 \%$ and $50.8 \%$, respectively (see Table 5.5). These results indicate that, despite the lower molecular weight, polymers synthesized via DAP show a better OPV performance than Stille synthesized polymer, which is consistent with their OFET mobility results (see section 5.2.5). This difference might be due to the end-group effect present in Stille synthesized polymers. ${ }^{297,298,308-310}$ Therefore, this work shows that DAP synthesized polymers could provide a good quality with better performance polymers in both OFET and OPV devices, despite their low $M_{\mathrm{n}}$ value. 
Table 5.5. Photovoltaic performance of PDFBT-Th4 polymers (E3a-DAP and Stille-B2).

\begin{tabular}{|c|c|c|c|c|c|}
\hline PDFBT-Th $_{4}$ & $\mathrm{~J}_{\mathrm{sc}}\left(\mathrm{mA} \mathrm{cm} \mathrm{cm}^{-2}\right)$ & $V_{\text {oc }}(V)$ & FF (\%) & PCE (\%) ${ }^{\max }$ & PCE (\%) ${ }^{\text {aver }}$ \\
\hline E3a-DAP & 12.39 & 0.77 & 45.7 & 4.36 & 4.12 \\
\hline Stille-B2 & 12.23 & 0.52 & 50.8 & 3.23 & 3.09 \\
\hline
\end{tabular}

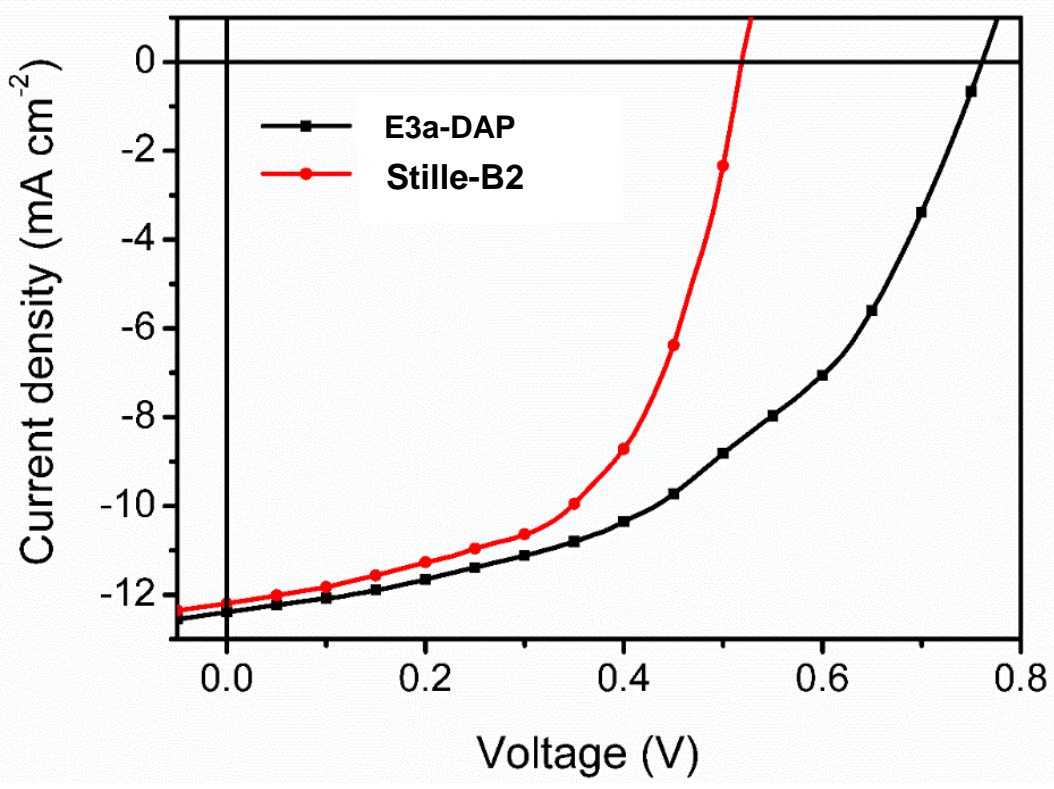

Figure 5.9. Current density-voltage curves of photovoltaic devices with active layers of PDFBT-Th4 (E3a-DAP and Stille-B2 polymers) and $\mathrm{PC}_{71} \mathrm{BM}$ acceptor (1:1.2 by weight) with $3 \%$ of DIO additive at $800 \mathrm{rpm}$ spin speed.

\subsection{Conclusion}

In summary, direct arylation polymerization (DAP) has been used in the synthesis of 5,6-difluoro-2,1,3-benzothiadiazole (DFBT) based polymer, PDFBT- 
Th4, in comparison with a polymer synthesized by Stille polymerization method. A series of DAP optimization have led to a high $M_{\mathrm{n}}$ up to $17 \mathrm{kDa}$ for the catalytic condition of $\mathrm{Pd}(\mathrm{OAc})_{2} /(\mathrm{o}-\mathrm{MeOPh})_{3} \mathrm{P} / \mathrm{PivOH} / \mathrm{K}_{2} \mathrm{CO}_{3} / \mathrm{o}-\mathrm{xylene}$. From a series of optimization reaction, $\mathrm{Pd}$ catalyst in $(+2)$ oxidation state is more reactive towards high degree polymerization than Pd (0) catalyst. PDFBT-Th 4 has displayed a strong interchain aggregation behavior in a film state, which is greatly enhanced for DAP synthesized polymer after thermal annealing. Polymer synthesized via DAP have displayed a better FET charge transport behavior and PCE of $4.12 \%$ despite their lower molecular weight as compared with polymer synthesized by Stille polymerization method. Therefore, this chapter represents a key step further to broadening the scope of high-quality semiconducting polymers that can be synthesized via DAP.

\section{The content in Chapter 5 has been published in}

- J. Polym. Sci. A Polym. Chem. (2017): Just Accepted 


\section{CHAPTER 6}

\section{Synthesis of Low-Bandgap Donor-Acceptor}

\section{Copolymer Based on 5H-Dithieno[3,2-b:2',3'-d]}

pyran via Direct Arylation Polymerization

\subsection{Introduction}

In Chapter 5, the synthesis of 5,6-difluoro-2,1,3-benzothiadiazole (DFBT) based donor-acceptor (D-A) conjugated polymer via direct arylation polymerization (DAP) was presented and shown interesting optoelectronic properties as compared with their analogs polymers. In this chapter, this direct $(\mathrm{C}-\mathrm{H})$ arylation polymerization method is extended to the synthesis of another DFBT based lowbandgap D-A conjugated polymer. As described in the previous chapters, copolymerizing electron donor and acceptor moieties is a successful strategy to tailor the optoelectronic properties of conjugated copolymers. ${ }^{161,209,311-313}$ Combining this technique with the inclusion of fused-ring structures in the conjugated backbone, such as cyclopentadithiophenes, dithienosiloles, dithienopyrroles, and 
thienothiophenes could enhance planarity and thus extended the conjugation length..$^{85,314-318}$

Recently, Dou et al. have synthesized a new strong donor moiety, 5Hdithieno[3,2-b:2',3'-d]pyran (DTP), which possess a fused-ring system and copolymerized with DFBT acceptor moiety via Stille polymerization method to give a low bandgap (1.38 eV) novel D-A conjugated polymer (DFBT-alt-DTP). This polymer exhibited a high power conversion efficiency (PCE) of $8 \%$ in its corresponding organic photovoltaic devices. ${ }^{297}$ Despite the encouraging performances of DFBT-alt-DTP polymers and other polymers containing thiophene fused-ring system, the potential of their synthetic scale up has rarely mentioned in the literature. This is particularly due to the disadvantages posed by traditional C-C coupling techniques, such as Suzuki-Miyaura and Migita-Kosugi-Stille couplings. 274-277 As mentioned in Chapter 1 and 5, these synthetic protocols require a tedious preactivation of $\mathrm{C}-\mathrm{H}$ bonds by toxic and flammable precursors (boronic acid or trialkyltin) and also the polycondensation produces a stoichiometric amount of toxic byproducts. As a consequence, an environmentally friendly, scalable reaction which retains high yield for mass production of these conjugated polymers is required for the future large-scale production and commercialization of organic electronics devices (OFETs, OPVs).

As mentioned in the previous chapters, recently, a new coupling chemistry called direct $(\mathrm{C}-\mathrm{H})$ arylation polymerization (DAP) has attracted a tremendous 
attention to synthesize $\pi$-conjugated polymers. DAP involves the coupling of an aryl halide monomer directly with another arene monomer without the need for preactivation of C-H bonds. ${ }^{278-280}$ Therefore, it is an economically efficient and environmentally benign approach compared to the traditional coupling methods such as Stille and Suzuki couplings. ${ }^{61,281-284}$

To date, a large library of donor and acceptor moieties are available for the synthesis of D-A conjugated polymers for organic electronics applications. For instance, some of the largely studied donor moieties are carbazole $(\mathrm{Cz})$, benzodithiophene (BDT), dithienosilole(DTS), dithienogermole (DTG), and cyclopentadithiophene (CPDT) units. A structurally similar donor moiety, 5Hdithieno[3,2-b:2',3'-d]pyran (DTP) has an electron-donating oxygen atom in the pyran ring, which makes DTP a strong electron donor moiety than the other mentioned moieties in Figure 6.1. Therefore, copolymerizing DTP with a strong acceptor moiety like DFBT could give a low bandgap D-A conjugated polymer which possesses UV-vis absorption spectra extended to NIR region.

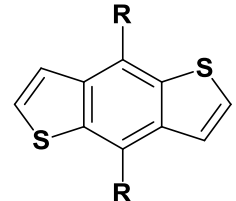

BDT

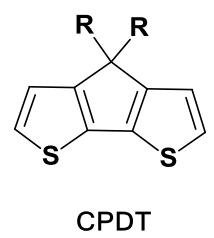

CPDT

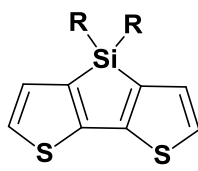

DTS

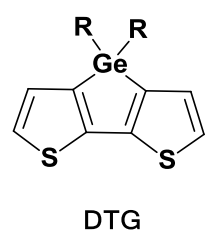

DTG

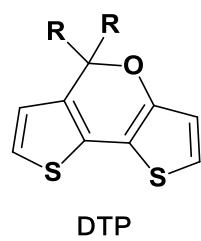

DTP

Figure 6.1. Chemical structures of BDT, CPDT, DTS, DTG, and DTP units. $R=$ alkyl chains 
This chapter presents the DAP synthesis of 5H-dithieno[3,2-b:2',3'-d]pyran (DTP) based low bandgap D-A conjugated polymers, poly(5,6-difluoro-2,1,3benzothiadiazole-alt-dithienopyran) PDFBT-alt-DTP with solubilizing alkyl chain attached on the donor moiety. Its synthesis optimization towards high molecular weight has been carried out through changing the different combinations of reaction time, temperature, and concentration of monomers. The physicoelectrochemical properties of the obtained polymers were characterized to better understand the structure-property relations of the polymers using ${ }^{1} \mathrm{H}-\mathrm{NMR}$, TGA, UV-vis-NIR spectra, and cyclic voltammetry experiments. The charge carrier property of the target polymer (Entry with high molecular weight) was measured by its OFET devices ambient conditions. 






DBrDFBT

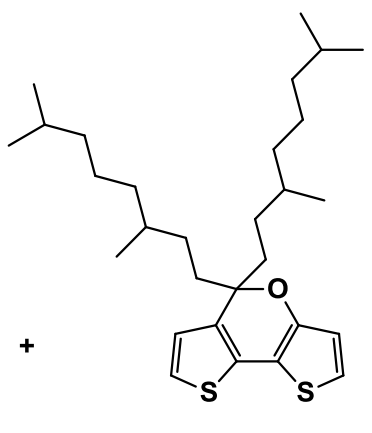

26

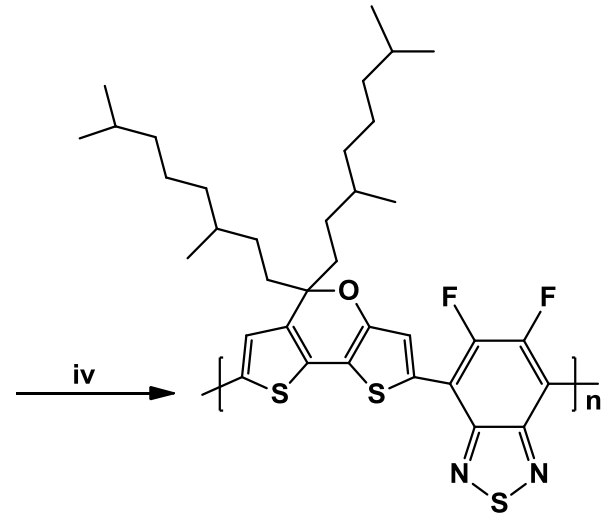

PDFBT-DTP

Scheme 10: Synthetic route to PDFBT-DTP polymer via DAP. (i) $\mathrm{NaBO}_{3} \cdot \mathrm{H}_{2} \mathrm{O}$, $\mathrm{CHCl}_{3}$, TFA, R.T., $27 \%$ yiled (compound 24). (ii) $\mathrm{BrMgC}_{10} \mathrm{H}_{21}, \mathrm{THF},<-20{ }^{\circ} \mathrm{C}, 86 \%$ yield (compound 25). (iii) p-TSA. $\mathrm{H}_{2} \mathrm{O}$, toluene, $110{ }^{\circ} \mathrm{C}, 95 \%$ yield (compound 26). (iv) DAP Protocol (See Table 6.1).

\subsection{Experimental}

Materials. Unless stated otherwise, all chemicals and solvents were obtained from commercial sources and used without further purification unless otherwise stated. 4H-cyclopenta[1,2-b:5,4-b']dithiophen-4-one were commercially obtained 
from SunaTech Inc. and 4,7-dibromo-5,6-difluorobenzo[c][1,2,5]thiadiazole (DBrDFBT) were obtained from Derthon optoelectronics materials. Compound 24, $\mathbf{2 5}, \boldsymbol{\&} \mathbf{2 6}$ were synthesized according to reported procedures. ${ }^{297}$

\subsubsection{Synthesis of monomer}

\section{H-dithieno[3,2-b:2',3'-d]pyran-5-one $(24)^{297}$}

4H-cyclopenta[1,2-b:5,4-b']dithiophen-4-one (CPD) (1 g, $5.4 \mathrm{mmol}$ ) was dissolved in the mixture of trifluoroacetic acid and chloroform solvents $(10 \mathrm{~mL}$ each $(1: 1 \mathrm{v} / \mathrm{v}))$, followed by the addition of sodium perborate monohydrate $(1.28 \mathrm{~g}, 12.6$ mmol) in one portion and stirring for $45 \mathrm{~min}$ at room temperature. The chloroform extract was purified by column chromatography using a mixture of hexane and DCM solvents (1:1, v/v) as eluent to get Compound $24(0.3 \mathrm{~g}$, yield 27\%) as a white solid.

${ }^{1} \mathrm{H}-\mathrm{NMR}\left(\mathrm{CDCl}_{3}, 300 \mathrm{MHz}\right): 7.10(\mathrm{~d}, 1 \mathrm{H}), 7.26(\mathrm{~d}, 1 \mathrm{H}), 7.42$ (d, 1H), 7.63 (d, $1 \mathrm{H})$.

\section{3'-(9-hydroxy-2,6,12-trimethylheptadecan-9-yl)-[2,2'-bithiophen]-3-ol (25). ${ }^{297}$}

Compound 24 (0.3 g, $1.43 \mathrm{mmol})$ was dissolved in $7.5 \mathrm{~mL}$ of anhydrous tetrahydrofuran (THF) solvent under a nitrogen atmosphere. After cooling the reaction mixture at $(-20 \text { to }-40)^{\circ} \mathrm{C}$, Grignard reagent (3,7-dimethyloctylmagnesium bromide of $1 \mathrm{M}$ diethyl ether solution) of $3.2 \mathrm{~mL}$ was added dropwise. Then it was stirred at room temperature for $6 \mathrm{~h}$. Water $(10 \mathrm{~mL})$ was added to quench the reaction, 
followed by ethyl acetate extraction and purification via short column chromatography to give Compound $\mathbf{2 5}$ in $\mathbf{8 6 \%}$ yield as a dark yellow oil.

\section{5-(3,7-dimethyloctyl)-5-(3-methyloctyl)-5H-dithieno[3,2-b:2',3'-d]pyran (26). ${ }^{297}$}

Compound 25 (0.61 g, $1.24 \mathrm{mmol})$ was dissolved in $10 \mathrm{~mL}$ of dry toluene under nitrogen atmosphere followed by sodium p-toluenesulfonic acid monohydrate (40 $\mathrm{mg}$ ) addition and stirring at $100^{\circ} \mathrm{C}$ for $1.5 \mathrm{~h}$. The column chromatography using hexane as eluent of the toluene extract gives Compound $\mathbf{2 6}$ in $95 \%$ of $0.56 \mathrm{~g}$, as yellow oil.

${ }^{1} \mathrm{H}-\mathrm{NMR}\left(\mathrm{CDCl}_{3}, 300 \mathrm{MHz}\right): 0.85$ (s, 12H), 0.89 (s, 6H), $1.57-1.01$ (br, 20H), $1.87(\mathrm{~m}, 4 \mathrm{H}), 6.67-6.66(\mathrm{~m}, 2 \mathrm{H}), 6.97-6.95(\mathrm{~d}, 1 \mathrm{H}), 7.03-7.01(\mathrm{~d}, 1 \mathrm{H})$

\subsubsection{General synthesis procedure for PDFBT-DTP polymer via direct arylation polymerization (DAP)}

A mixture of 5-(3,7-dimethyloctyl)-5-(3-methyloctyl)-5H-dithieno[3,2b:2',3'-d]pyran (Compound 26) (1 equiv.) and 4,7-dibromo-5,6-difluorobenzo[c] [1,2,5]thiadiazole (DBrDFBT) (1equiv.), palladium source, ligand, base, $\mathrm{PivOH}$, and o-xylene was added in a reaction vial containing a magnetic stirring bar under nitrogen atmosphere. The vial sealed with an aluminum cap with PTFE/silicone septum and then heated in a preheated oil bath at $100{ }^{\circ} \mathrm{C}$ except for Entry 1, E1-DTP heated at $140{ }^{\circ} \mathrm{C}$. After being cooled to room temperature, the reaction mixture was diluted with $\mathrm{CHCl}_{3}$ and added dropwise to $150 \mathrm{~mL}$ of methanol, precipitates were 
collected by filtration and then subjected to Soxhlet extraction with methanol, acetone, hexane, and chloroform sequentially each for $24 \mathrm{~h}$. The hexane and/or chloroform fraction was concentrated and precipitated into methanol. The precipitates were collected by filtration and dried under vacuum before further characterization.

Entry 1: $\mathrm{Pd}_{2}(\mathrm{dba})_{3}(5 \mathrm{~mol} \%),(o-\mathrm{MeOPh})_{3} \mathrm{P}(10 \mathrm{~mol} \%), \mathrm{K}_{2} \mathrm{CO}_{3}$ (4 equiv.), PivOH (50 mol\%), and o-xylene solvent $(0.1 \mathrm{M})$ at $140{ }^{\circ} \mathrm{C}$. Yield: $48 \%$ from $\mathrm{CHCl}_{3}$ fraction.

Entry 2: $\mathrm{Pd}_{2}(\mathrm{dba})_{3}(5 \mathrm{~mol} \%),(o-\mathrm{MeOPh})_{3} \mathrm{P}(10 \mathrm{~mol} \%), \mathrm{K}_{2} \mathrm{CO}_{3}$ (4 equiv.), PivOH (50 mol\%), and o-xylene solvent $(0.2 \mathrm{M})$ at $100{ }^{\circ} \mathrm{C}$. Yield: $88 \%$ from $\mathrm{CHCl}_{3}$ fraction.

Entry 3: $\mathrm{Pd}(\mathrm{AoC})_{2}(5 \mathrm{~mol} \%),(o-\mathrm{MeOPh})_{3} \mathrm{P}(10 \mathrm{~mol} \%), \mathrm{K}_{2} \mathrm{CO}_{3}$ (4 equiv.), PivOH (50 mol\%), and o-xylene solvent $(0.2 \mathrm{M})$ at $100{ }^{\circ} \mathrm{C}$. Yield: $80 \%$ from $\mathrm{CHCl}_{3}$ fraction.

Entry 4: Herrmann's catalyst (5 mol\%), (o-MeOPh) ${ }_{3} \mathrm{P}(10 \mathrm{~mol} \%), \mathrm{K}_{2} \mathrm{CO}_{3}(4$ equiv.), PivOH (50 mol\%), and o-xylene solvent $(0.2 \mathrm{M})$ at $100{ }^{\circ} \mathrm{C}$. no polymerization.

Entry 5: $\mathrm{Pd}_{2}(\mathrm{dba})_{3}(5 \mathrm{~mol} \%),(o-\mathrm{MeOPh})_{3} \mathrm{P}(10 \mathrm{~mol} \%), \mathrm{K}_{2} \mathrm{CO}_{3}$ (4 equiv.), PivOH (50 mol\%), and o-xylene solvent $(0.3 \mathrm{M})$ at $100{ }^{\circ} \mathrm{C}$. Yield: $83 \%$ from $\mathrm{CHCl}_{3}$ 
fraction.

Entry 6: $\mathrm{Pd}_{2}(\mathrm{dba})_{3}(5 \mathrm{~mol} \%),(o-\mathrm{MeOPh})_{3} \mathrm{P}(10 \mathrm{~mol} \%), \mathrm{K}_{2} \mathrm{CO}_{3}$ (4 equiv.), PivOH (50 mol\%), and o-xylene solvent $(0.2 \mathrm{M})$ at $100{ }^{\circ} \mathrm{C}$. Yield: $15 \%$ from $\mathrm{CHCl}_{3}$ fraction.

\subsubsection{GPC traces for polymers and NMR spectra}

\subsubsection{GPC traces for polymers}
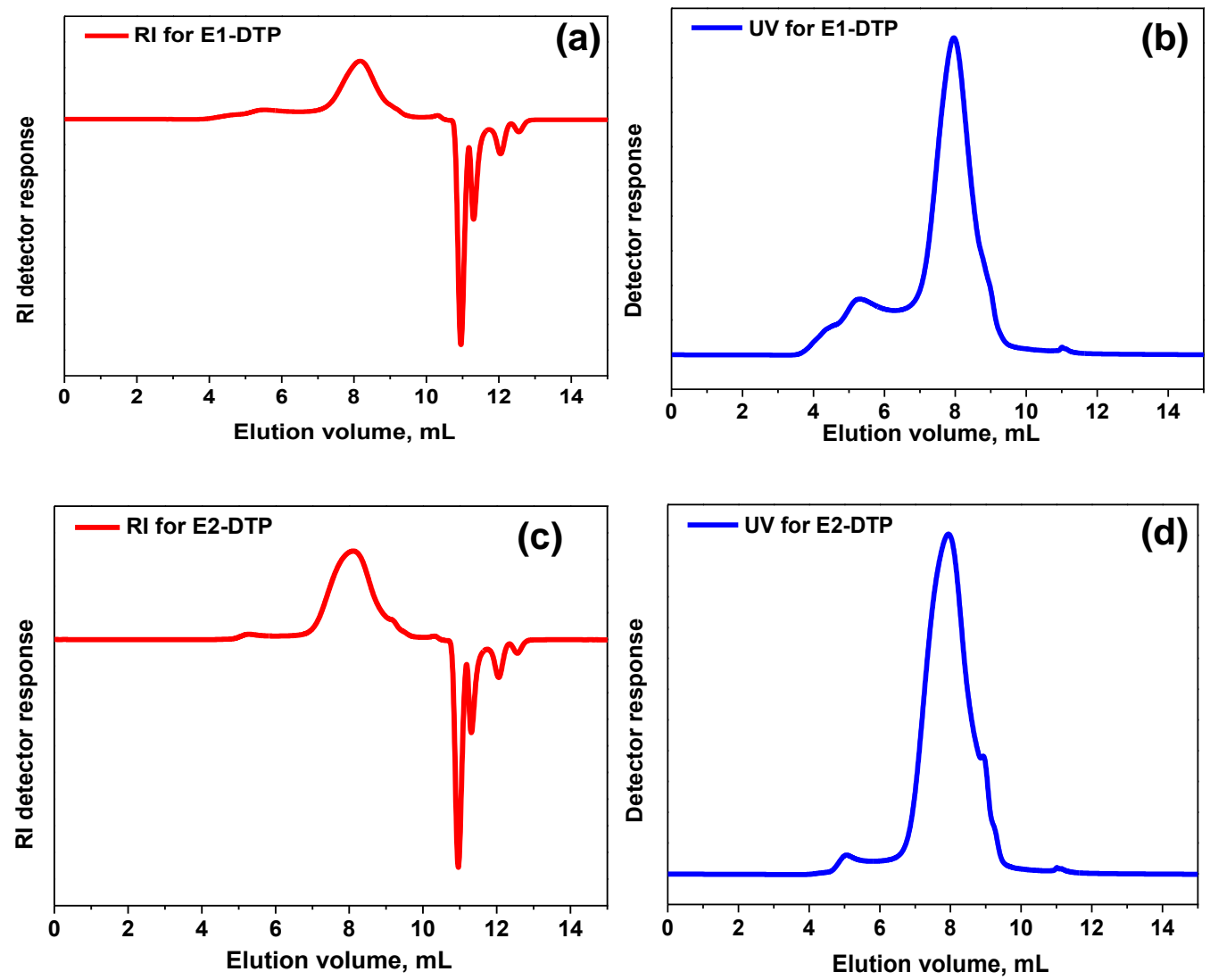

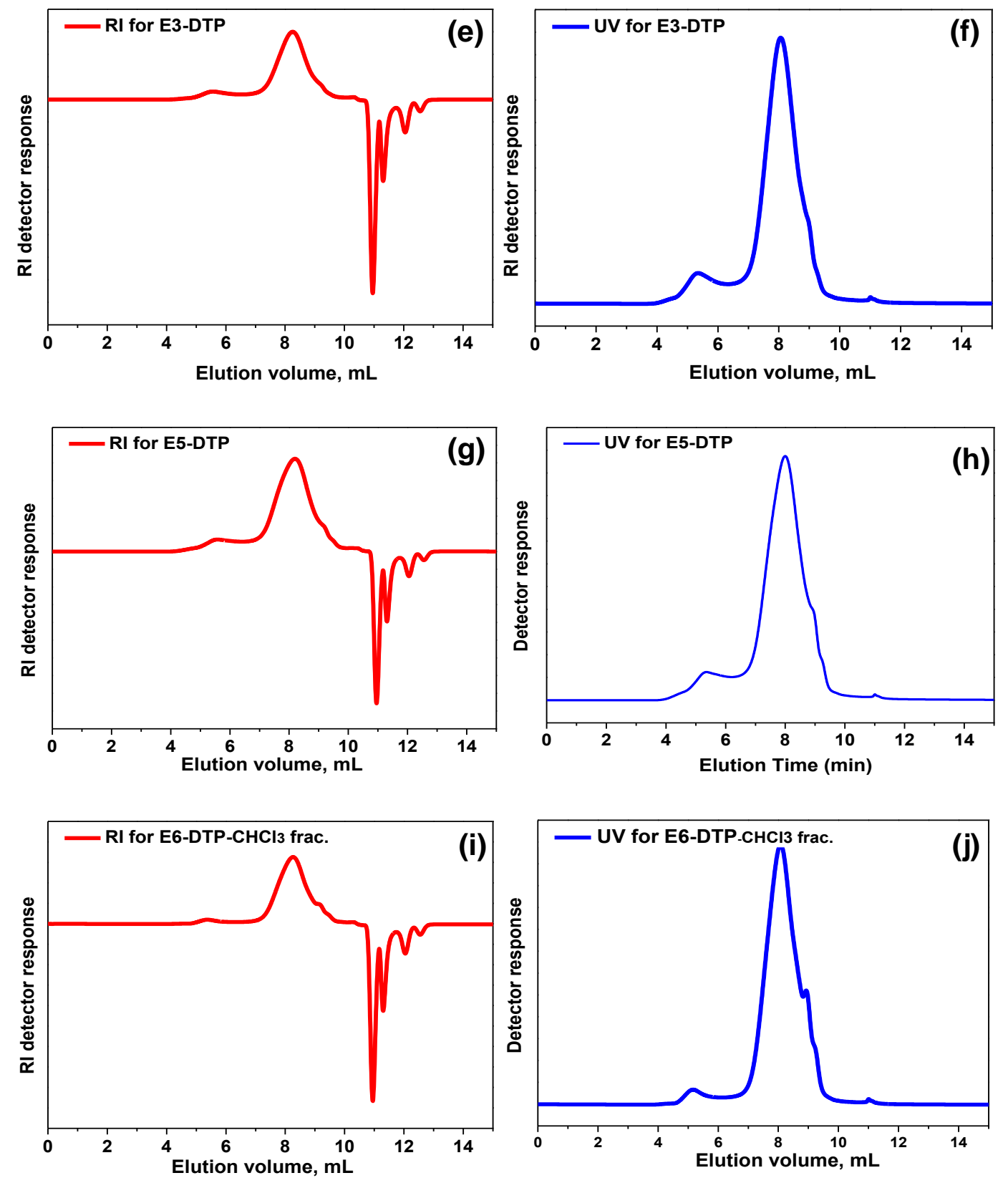

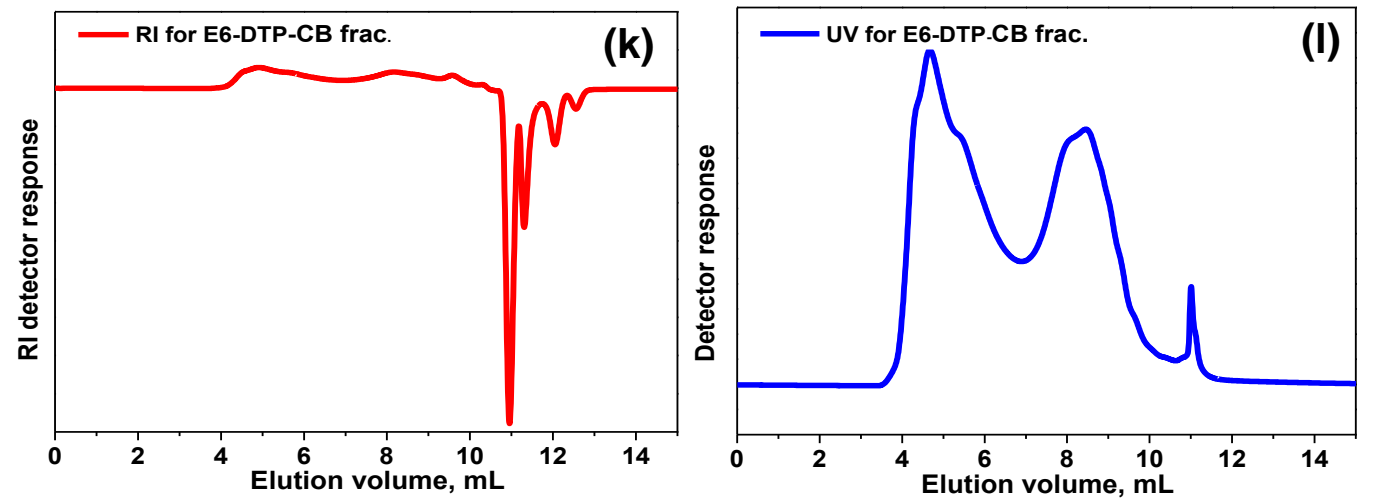

Figure 6.2. GPC traces for PDFBT-DTP polymers of E1-DTP, E2-DTP, E3-DTP, E5-DTP, E6-DTP of $\mathrm{CHCl}_{3}$ frac. and E6-DTP of CB frac. (using THF as eluent at r.t.) from RI (a, c, e, g, i, and k) and UV (550 nm) (b, d, f, h, j, and l) detectors, respectively.

\subsubsection{NMR spectra of monomers}

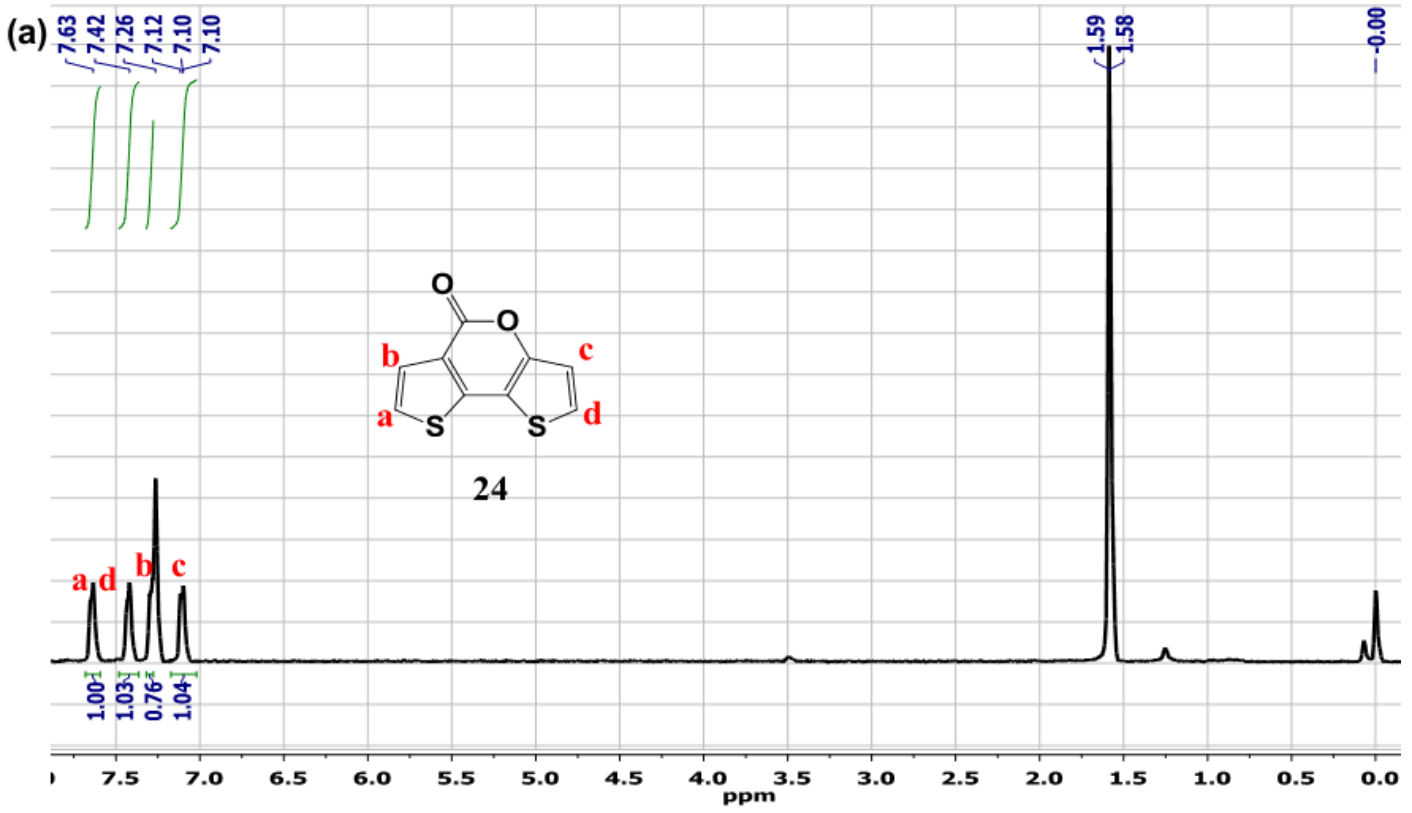




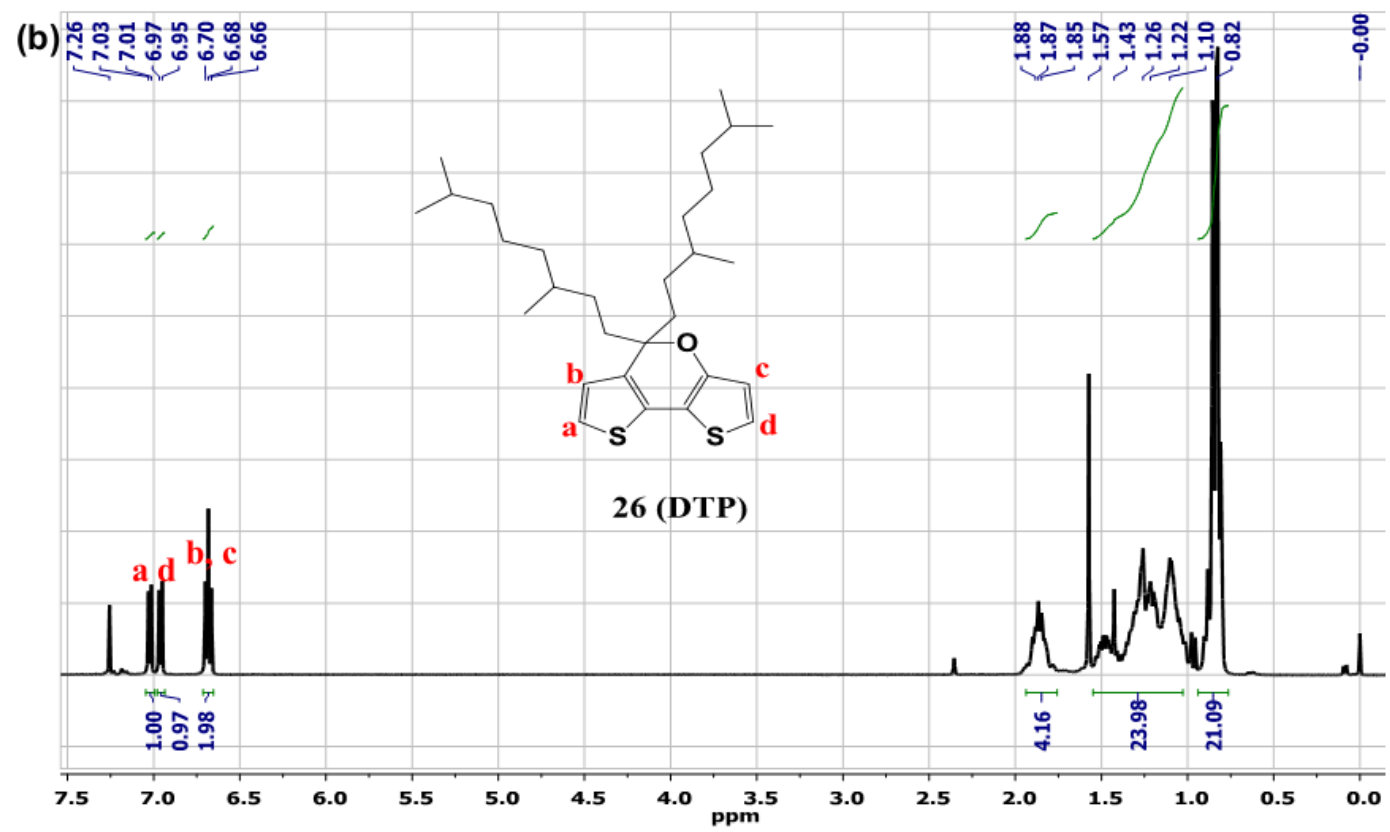

Figure 6.3. ${ }^{1} \mathrm{H}$ NMR (300 MHz) spectra for compound 24(a) and 26 (b) in $\mathrm{CDCl}_{3}$ solvent at R.T.

\subsection{Results and Discussion}

\subsubsection{Synthesis and structural characterization}

The synthetic routes of donor moiety 5-(3,7-dimethyloctyl)-5-(3-methyloctyl)5H-dithieno[3,2-b:2',3'-d]pyran (Compound 26, DTP) and its copolymerization with 5,6-Difluoro-2,1,3-benzothiadiazole (DFBT) are shown in Scheme 10. The syntheses of Compound $\mathbf{2 4}$ to $\mathbf{2 6}$ were conducted according to reported procedure. ${ }^{297}$ CPD oxidation by using $\mathrm{NaBO}_{3} \cdot \mathrm{H}_{2} \mathrm{O}$ provides Compound 24 in $27 \%$ yield. As described by Dou et al. ${ }^{297}$ the possible oxidation on the other positions of CPD might be the reason for its low yield. Then, room temperature treatment of Compound $\mathbf{2 4}$ 
with excess Grignard reagent (3,7-dimethyloctylmagnesium bromide) introduces an alkyl side chain to give Compound 25 with $86 \%$ yield. Then, followed by ring closing reaction via p-TSA $\cdot \mathrm{H}_{2} \mathrm{O}$ treatment in dry toluene gives Compound $\mathbf{2 6}$ in $95 \%$ yield.

The DAP synthetic route to PDFBT-DTP polymer is shown in Scheme 10, where Compound 26 and 4,7-dibromo-5,6-difluorobenzo[c][1,2,5]thiadiazole (DBrDFBT) were reacted under different DAP reaction conditions aiming to get good quality, high molecular weight and reaction yield of PDFBT-DTP polymer. Therefore, the optimization of DAP reaction was carried out by changing conditions, such as palladium-catalyst, concentration of monomers, and reaction temperature (see Table 6.1) and the progress of reaction was monitored by the color change from dark red to dark cyan (which is the characteristics color of the PDFBT-DTP polymer as reported in literature). ${ }^{297}$

First, Compound 26 and DBrDFBT were reacted in the presence of $\mathrm{Pd}_{2}(\mathrm{dba})_{3}$ catalyst, (o-MeOPh) ${ }_{3} \mathrm{P}$ ligand, $\mathrm{PivOH}$ additive, $\mathrm{K}_{2} \mathrm{CO}_{3}$ base, and o-xylene $(0.1 \mathrm{M})$ at $140{ }^{\circ} \mathrm{C}$ for $24 \mathrm{~h}$ (Entry E1-DTP), which gives PDFBT-DTP polymer in a low yield of $48 \%\left(M_{\mathrm{n}}=17.6 \mathrm{kDa}\right)$ from the chloroform fraction. The effort to further increase the yield and molecular weight was carried out by increasing the concentration to 0.2 $\mathrm{M}$ and decreasing the reaction temperature to $100{ }^{\circ} \mathrm{C}$ (Entry E2-DTP) to give PDFBT-DTP polymer with an increased yield of $88 \%$ from chloroform fraction with comparable molecular weight $\left(M_{\mathrm{n}}=17.7 \mathrm{kDa}\right)$ with E1-DTP. Further increase in concentration to 0.3 and $0.5 \mathrm{M}$ in Entry E5-DTP and E6-DTP, respectively, did not 
show improvement in $M_{\mathrm{n}}$. However, in Entry E6-DTP the yield in chloroform fraction greatly decreased to $15 \%$ with the formation of an insoluble residue which was later completely dissolved in chlorobenzene, which can possibly be attributed to side reactions, such as crosslinking or branching occurred at 3- and 6-positions of Compound 26.

$\mathrm{Pd}(\mathrm{OAc})_{2}$ and Herrmann's catalyst were employed to gain more information about the effect of a catalyst on the DAP reaction between Compound $\mathbf{2 6}$ and DBrDFBT. Thus, in Entry E3-DTP, $\mathrm{Pd}(\mathrm{OAc})_{2}$ gave polymer in a relatively lower molecular weight $\left(M_{\mathrm{n}}=14.2 \mathrm{kDa}\right)$, whereas Herrmann's catalyst in Entry E4-DTP gave no polycondensation product, where the color of the reaction mixture after 24 $\mathrm{h}$ was dark purple and no precipitate was obtained from methanol. Based on the aforementioned optimization results, the combination of $\operatorname{Pd}_{2}(\mathrm{dba})_{3} /(\mathrm{o}-$ $\mathrm{MeOPh})_{3} \mathrm{P} / \mathrm{PivOH} / \mathrm{K}_{2} \mathrm{CO}_{3} / \mathrm{o}-\mathrm{xylene}(0.2 \mathrm{M})$ of Entry E2-DTP is found to be the best DAP reaction condition for the synthesis of high molecular weight PDFBT-DTP polymer. These results indicate that it could be possible to synthesize PDFBT-DTP polymer via DAP, despite the lower $M_{\mathrm{n}}$ of PDFBT-DTP polymer obtained by DAP as compared with that of PDFBT-DTP polymer obtained by Stille polymerization method as reported by Dou et al. $\left(M_{\mathrm{n}}=28.5 \mathrm{kDa}\right) .{ }^{297}$ Based on this findings, we suggest that the molecular weight of PDFBT-DTP polymer could be further increased via changing different DAP reaction conditions like the type of catalyst, solvent, concentration, ligand, base and reaction time. 
Table 6.1. DAP optimization entries for synthesis the PDFBT-DTP polymer.

\begin{tabular}{|c|c|c|c|c|c|c|}
\hline $\begin{array}{l}\text { Entry } \\
\text { no. }\end{array}$ & Catalyst & $\begin{array}{l}\text { Concentration } \\
\text { (M) }\end{array}$ & $\begin{array}{l}\text { Temperature } \\
\left({ }^{\circ} \mathrm{C}\right)\end{array}$ & $\begin{array}{l}\text { Yield } \\
(\%)\end{array}$ & $\begin{array}{l}{ }^{\mathrm{d}} M_{\mathbf{n}} \\
(\mathbf{k D a})\end{array}$ & PDI \\
\hline E1-DTP & $\mathrm{Pd}_{2}(\mathrm{dba})_{3}$ & 0.1 & 140 & 48 & 17.6 & 2.1 \\
\hline E2-DTP & $\mathrm{Pd}_{2}(\mathrm{dba})_{3}$ & 0.2 & 100 & 88 & 17.7 & 2.5 \\
\hline E3-DTP & $\mathrm{Pd}(\mathrm{OAc})_{2}$ & 0.2 & 100 & 80 & 14.2 & 2.3 \\
\hline E4-DTP & Hermann & 0.2 & 100 & ${ }^{c}-$ & - & - \\
\hline E5-DTP & $\mathrm{Pd}_{2}(\mathrm{dba})_{3}$ & 0.3 & 100 & 83 & 15.3 & 2.6 \\
\hline E6-DTP & $\mathrm{Pd}_{2}(\mathrm{dba})_{3}$ & 0.5 & 100 & $\begin{array}{l}{ }^{\mathrm{a}} 15 \\
\text { b72 }\end{array}$ & $\begin{array}{l}15.2 \\
\mathrm{e}_{-}\end{array}$ & 1.9 \\
\hline
\end{tabular}

${ }^{a}$ Yield of the polymer from chloroform fraction. ${ }^{\text {b } Y i e l d ~ o f ~ t h e ~ p o l y m e r ~ f r o m ~}$ chlorobenzene fraction. ${ }^{\mathrm{c}}$ No precipitate in methanol. ${ }^{\mathrm{d}}$ Measured by GPC with polystyrene as standard and THF as eluent at room temperature. ${ }^{e}$ Very broad bimodal GPC trace was obtained. For all entries; $\mathrm{PiVOH}$ additive, $\mathrm{P}(\mathrm{o}-\mathrm{MeOPh})_{3}$ ligand, and $\mathrm{K}_{2} \mathrm{CO}_{3}$ base were used. The reaction time of $24 \mathrm{~h}$.

To characterize the chemical structure of PDFBT-DTP polymers synthesized via DAP, high temperature $\left(100{ }^{\circ} \mathrm{C}\right){ }^{1} \mathrm{H}-\mathrm{NMR}$ spectra in $\mathrm{C}_{2} \mathrm{D}_{2} \mathrm{Cl}_{4}$ as the solvent were collected (see Figure 6.4). The extra little peaks found in the ${ }^{1} \mathrm{H}-\mathrm{NMR}$ spectra can be related to different end groups from the monomers. For instance, as shown in Figure 6.4, there are very tiny peaks around 7.52, 7.38 and $6.99 \mathrm{ppm}$, which could 
correspond to end groups from Compound $\mathbf{2 6}$ in the polymer. The selectivity of $(\alpha) \mathrm{C}$ $\mathrm{H}$ over $(\beta) \mathrm{C}-\mathrm{H}$ protons on Compound $\mathbf{2 6}$ is good as indicated by the presence of (ß)C-H protons around 7.93 and $7.88 \mathrm{ppm}$ in the ${ }^{1} \mathrm{H}-\mathrm{NMR}$ spectra of all entries of the DAP polymers. As described by Dou et al., ${ }^{297}$ it was difficult to get highly pure stannylated monomer of Compound 26 in the synthesis of PDFBT-DTP by Stille polymerization and thus affected the quality of PDFBT-DTP polymer and it's device performance unless it was end-caped with bromobenzene. Therefore, employing direct arylation polymerization for the synthesis PDFBT-DTP polymer might avoid stannylation related problems. 


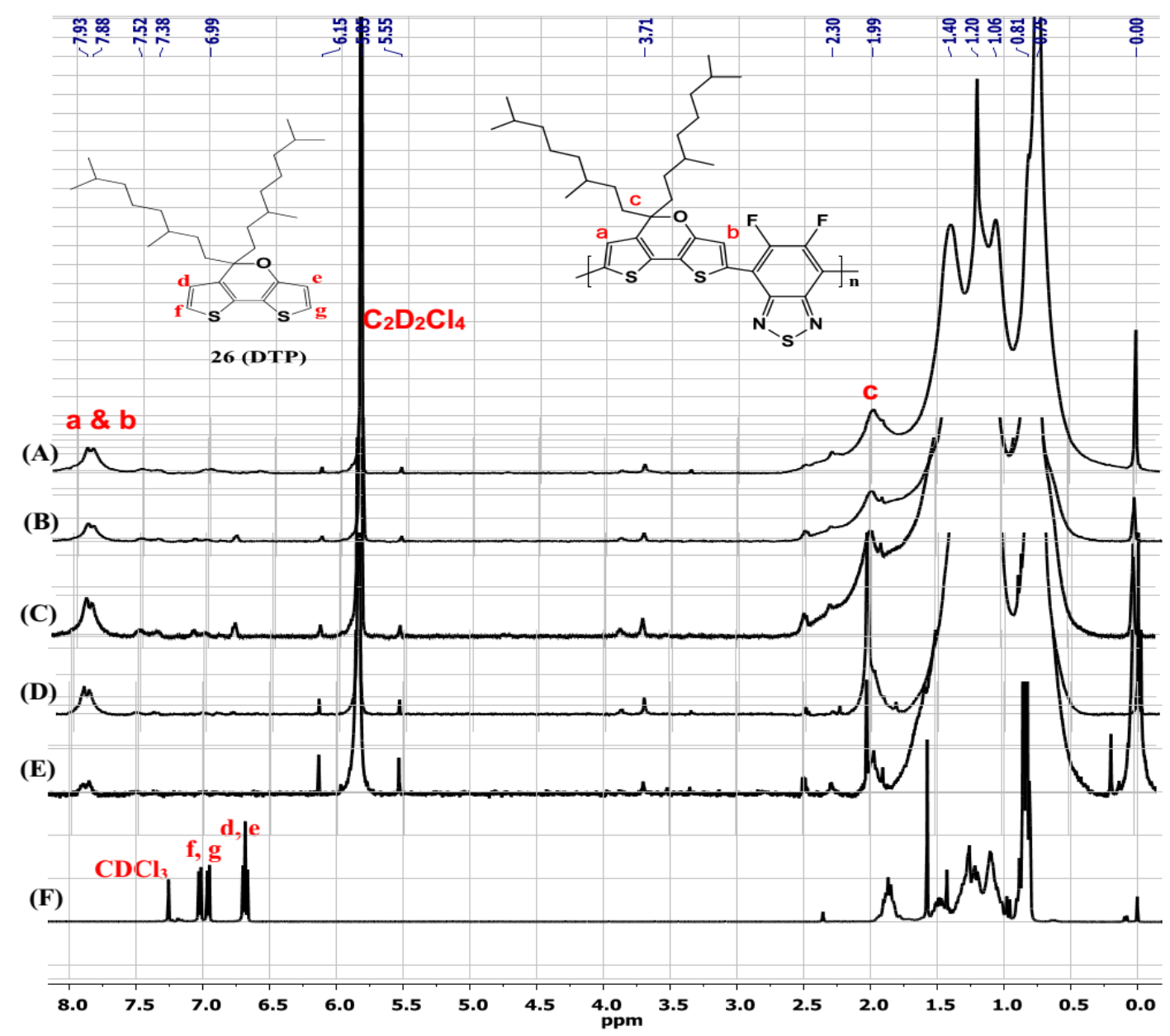

Figure 6.4. ${ }^{1} \mathrm{H}-\mathrm{NMR}$ spectra of the PDFBT-DTP polymers synthesized via DAP, E1-DTP (a), E2-DTP (b), E3-DTP (c), E5-DTP (d), and E6-DTP-CB (d) at $100{ }^{\circ} \mathrm{C}$ in $\mathrm{C}_{2} \mathrm{D}_{2} \mathrm{Cl}_{4}$. DTP monomer (26) (f) in $\mathrm{CDCl}_{3}$ at r.t.

\subsubsection{Thermal Properties}

The thermal stability of PDFBT-DTP was investigated using thermogravimetric analysis (TGA) with a heating rate of $10{ }^{\circ} \mathrm{C} \min ^{-1}$ under $\mathrm{N}_{2}$ atmosphere. The decomposition temperature ( $T_{\mathrm{d}}$, corresponding to $5 \%$ weight loss) 
is located at $335^{\circ} \mathrm{C}$ for the PDFBT-DTP polymer with the highest $M_{\mathrm{n}}$ obtained by DAP (E2-DTP) (see Figure 6.5), demonstrating its sufficient high thermal stability for the application in organic electronic devices.

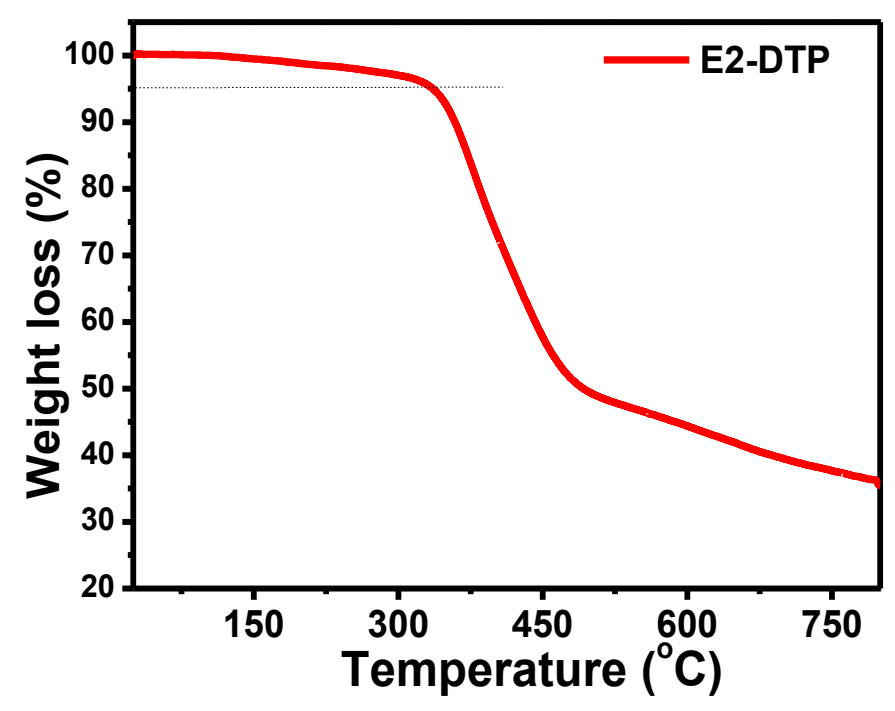

Figure 6.5. TGA plots of PDFBT-DTP (Entry E2-DTP) polymer with a heating rate of $10{ }^{\circ} \mathrm{C} \mathrm{min}{ }^{-1}$ under nitrogen.

\subsubsection{Optical properties}

The UV-vis absorption spectra for PDFBT-DTP polymers were acquired from chloroform solutions and chlorobenzene solution (for E6-DTP of chlorobenzene fraction) and spin-cast thin films prepared from their respective chlorobenzene solutions. The spectra are shown in Figure 6.6 and the relevant data are summarized in Table 6.2. Broad absorption bands across the visible region were observed for both solution and thin films. The absorption maxima for PDFBT-DTP polymers in solution (see Figure 6.6a) shows a red shift and a formation of intense shoulder peak 
as the molecular weight gets increased. E2-DTP has $\lambda_{\max }\left(\lambda_{\text {shoulder }}\right)$ of $711(776) \mathrm{nm}$ which is red-shifted ca.10-15 (14) nm as compared to the polymers from the other entries. E6-DTP from CB fraction shows a blue shifted $\lambda_{\max }$ absorption spectra of ca.60 nm, which might be due to the presence of crosslinking or branching in the polymer backbone.

The thin film of PDFBT-DTP polymers prepared by spin casting at $1500 \mathrm{rpm}$ on a quartz substrate from their respective chlorobenzene solution exhibit red-shifted absorbance (ca.10 nm for E2-DTP) as compared with its absorption spectra in chloroform. The thin-film spectra of the E2-DTP exhibit a shoulder peak at $780 \mathrm{~nm}$ with much-increased intensity as compared with its solution state, which is an indication of the presence of J-aggregation and strong intermolecular interaction in solid thin films. However, the intensity of the peak at $780 \mathrm{~nm}$ relative to that at 719 $\mathrm{nm}$ has weaker intensity in E2-DTP polymer compared to the similar polymer synthesized by Stille polymerization, ${ }^{297}$ this is probably due to the lower $M_{\mathrm{n}}$ of the DAP synthesized polymer. The corresponding optical bandgaps, $\mathrm{E}_{\mathrm{g}}{ }^{\mathrm{opt}}$ estimated from the onset absorption wavelength of their thin film spectra were calculated to be 1.41 $\mathrm{eV}$ for the E2-DTP polymer, which is almost equal to the one synthesized by Stille polymerization method as reported in the literature (see Table 6.2). ${ }^{297}$ The intramolecular charge transfer between the strong electron-withdrawing (DFBT) and electron-donating (DTP) moieties could be the reason for the low bandgap nature of the polymer. ${ }^{161,209,312,313,319}$ 
Table 6.2. Optical data of PDFBT-DTP polymers.

\begin{tabular}{|c|c|c|c|c|c|}
\hline \multirow{2}{*}{ Entry no. } & \multicolumn{2}{|c|}{ in $\mathrm{CHCl}_{3}$} & \multicolumn{3}{|c|}{ Film } \\
\hline & $\begin{array}{l}\lambda_{\max }\left(\lambda_{\text {sholder }}\right) \\
(\mathbf{n m})\end{array}$ & $\begin{array}{l}\lambda_{\text {onset }} \\
\text { (nm) }\end{array}$ & $\begin{array}{l}\lambda_{\max }\left(\lambda_{\text {sholder }}\right) \\
(\mathrm{nm})\end{array}$ & $\begin{array}{l}\lambda_{\text {onset }} \\
(\mathbf{n m})\end{array}$ & $\begin{array}{l}\text { Egopt } \\
(e V)\end{array}$ \\
\hline E1-DTP & 694 (767) & 860 & 704 (770) & 868 & 1.43 \\
\hline E2-DTP & $711(776)$ & 874 & $719(780)$ & 878 & 1.41 \\
\hline E3-DTP & $694(767)$ & 860 & $712(776)$ & 868 & 1.43 \\
\hline E5-DTP & 698 (767) & 860 & $712(780)$ & 876 & 1.42 \\
\hline E6-DTP (1) ${ }^{c}$ & $698(767)$ & 860 & $712(780)$ & 885 & 1.40 \\
\hline E6-DTP (2) ${ }^{d}$ & $638(767)^{e}$ & $860^{\mathrm{e}}$ & $664(786)$ & 873 & 1.42 \\
\hline
\end{tabular}

${ }^{\mathrm{a}}$ Film at room temperature. ${ }^{\mathrm{b}} \mathrm{Eg}^{\mathrm{opt}}(\mathrm{eV})=1240 / \lambda_{\text {onset }}[\mathrm{nm}] .{ }^{\mathrm{c}} \mathbf{E 6}$-DTP (1) is $\mathrm{CHCl}_{3}$ fraction and ${ }^{\mathrm{d}}$ E6-DTP (2) is chlorobenzene fraction. ${ }^{\mathrm{e}}$ E6-DTP (2) from chlorobenzene solution.


Figure 6.6. UV-vis-NIR absorption spectra of PDFBT-DTP polymers (a) in $\mathrm{CHCl}_{3}$ solution except E6-DTP (2) in chlorobenzene solution and (b) in thin film prepared 
by spin casting from their chlorobenzene solution. E6-DTP (1) is $\mathrm{CHCl}_{3}$ fraction and E6-DTP (2) is chlorobenzene fraction.

\subsubsection{Electrochemical properties}

The electrochemical properties of PDFBT-DTP polymers were investigated by cyclic voltammetry $(\mathrm{CV})$ analysis using ferrocene $\left(\mathrm{Fc} / \mathrm{Fc}^{+}\right)$as the standard (see Figure 6.7). It shows that PDFBT-DTP polymers oxidation and reduction potentials are reversible. Table 6.3, summarizes relevant data for PDFBT-DTP polymers. The oxidations were observed with an onset potential $\left(\mathrm{E}_{\mathrm{ox}}\right)$ at about 0.83 to $1.05 \mathrm{~V}$ vs. $\mathrm{Ag} / \mathrm{Ag}^{+}$and the onset reductions potentials $\left(\mathrm{E}_{\text {red }}\right)$ were observed at about -0.77 to $0.96 \mathrm{~V}$ vs. $\mathrm{Ag} / \mathrm{Ag}^{+}$. Thus, the $\mathrm{HOMO}^{\text {ele }} / \mathrm{LUMO}^{\text {ele }}$ energy levels were calculated by the following equation: $\mathrm{HOMO}^{\text {ele }} / \mathrm{LUMO}^{\text {ele }}=-\left(4.8-\mathrm{E}_{1 / 2}, \mathrm{Fc} / \mathrm{Fc}^{+}+\mathrm{E}_{\mathrm{ox}} / \mathrm{E}_{\mathrm{red}}\right) \mathrm{eV}$, where $\mathrm{E}_{1 / 2}, \mathrm{Fc} / \mathrm{Fc}^{+}=0.5 \mathrm{~V}$. Therefore, $\mathrm{HOMO}^{\text {ele }}$ of ca. $-5.2 \mathrm{eV}$ and $\mathrm{LUMO}^{\text {ele }}$ of ca.-3.4 to $3.5 \mathrm{eV}$ were calculated for all polymers from their corresponding $\mathrm{E}_{\mathrm{ox}}$ and $\mathrm{E}_{\mathrm{red}}$, respectively. The $\mathrm{LUMO}^{\text {opt }}$ calculated by using $\mathrm{LUMO}^{\text {opt }}=\mathrm{HOMO}^{\text {ele }}-\mathrm{E}_{\mathrm{g}}{ }^{\mathrm{opt}}$ and thus it is $-3.77 \mathrm{eV}$ for the E2-DTP polymer. The CV bandgap (Eg ${ }^{\text {ele }}$ ) of PDFBTDTP polymers is in the range of (1.71 to 1.97$) \mathrm{eV}$ which is slightly larger than that of the optical bandgap ( $\left.\mathrm{Eg}^{\text {ele }}\right)(1.40$ to 1.43$) \mathrm{eV}$. The discrepancy of Eg values lies within

the range of errors. ${ }^{320}$ The HOMO level $(5.18 \mathrm{eV})$ of E2-DTP polymer with a higher $M_{\mathrm{n}}$ is comparable with the HOMO level $(5.2 \mathrm{eV})$ of Stille synthesized polymer as reported by Dou et al. In addition to this, the CV bandgap $(1.76 \mathrm{eV})$ of E2-DTP 
polymer is slightly higher than that of Stille synthesized polymer $(1.62 \mathrm{eV}) .{ }^{297}$ This difference could be explained based on $M_{\mathrm{n}}$, where Stille synthesized polymer has higher $\left(M_{\mathrm{n}}=28 \mathrm{kDa}\right)$, thus which have higher conjugation length than E2-DTP polymer $\left(M_{\mathrm{n}}=17.6 \mathrm{kDa}\right)$.

Table 6.3. Electrochemical properties of PDFBT-DTP polymers.

\begin{tabular}{|c|c|c|c|c|c|c|}
\hline $\begin{array}{l}\text { PDFBT- } \\
\text { DTP }\end{array}$ & $\begin{array}{l}E_{o x} \\
(V)\end{array}$ & $\begin{array}{l}E_{\text {red }} \\
(V)\end{array}$ & $\begin{array}{l}{ }^{a} \mathrm{HOMO}^{\text {ele }} \\
(\mathrm{eV})\end{array}$ & $\begin{array}{l}{ }^{a} \mathrm{LUMO} \boldsymbol{O}^{\text {ele }} \\
(\mathrm{eV})\end{array}$ & $\begin{array}{l}{ }^{b} \mathrm{LUMO} \boldsymbol{O}^{\mathrm{opt}} \\
(\mathrm{eV})\end{array}$ & $\begin{array}{l}{ }^{c} E_{g} \text { ele } \\
(e V)\end{array}$ \\
\hline E1-DTP & 0.94 & -0.77 & -5.24 & -3.53 & -3.81 & 1.71 \\
\hline E2-DTP & 0.88 & -0.88 & -5.18 & -3.42 & -3.77 & 1.76 \\
\hline E3-DTP & 0.85 & -0.91 & -5.15 & -3.39 & -3.72 & 1.76 \\
\hline E5-DTP & 0.83 & -0.93 & -5.13 & -3.37 & -3.71 & 1.76 \\
\hline E6-DTP $(1)^{d}$ & 1.01 & -0.96 & -5.31 & -3.34 & -3.91 & 1.97 \\
\hline $\operatorname{E6-DTP}(2)^{e}$ & 1.05 & -0.87 & -5.35 & -3.43 & -3.93 & 1.92 \\
\hline \multicolumn{7}{|c|}{$\begin{array}{l}\mathrm{HOMO}^{\text {ele }} / \mathrm{LUMO}^{\text {ele }}=-\left(4.8-\mathrm{E}_{1 / 2}, \mathrm{Fc}, \mathrm{Fc}^{+}+\mathrm{E}_{\mathrm{ox}} / \text { red } \text {, onset }\right) .{ }^{\mathrm{b}} \mathrm{LUMO}^{\text {opt }}=\mathrm{Eg}^{\text {opt }}+ \\
\mathrm{HOMO}^{\text {ele }} .{ }^{\mathrm{c}} \mathrm{Eg}^{\text {ele }}=\mathrm{LOMO}^{\text {ele }}-\mathrm{HOMO}^{\text {ele }} .{ }^{\mathbf{d}} \mathbf{E 6} \text {-DTP }(1) \text { is } \mathrm{CHCl}_{3} \text { fraction and }{ }^{\text {eE6-DTP }}\end{array}$} \\
\hline
\end{tabular}



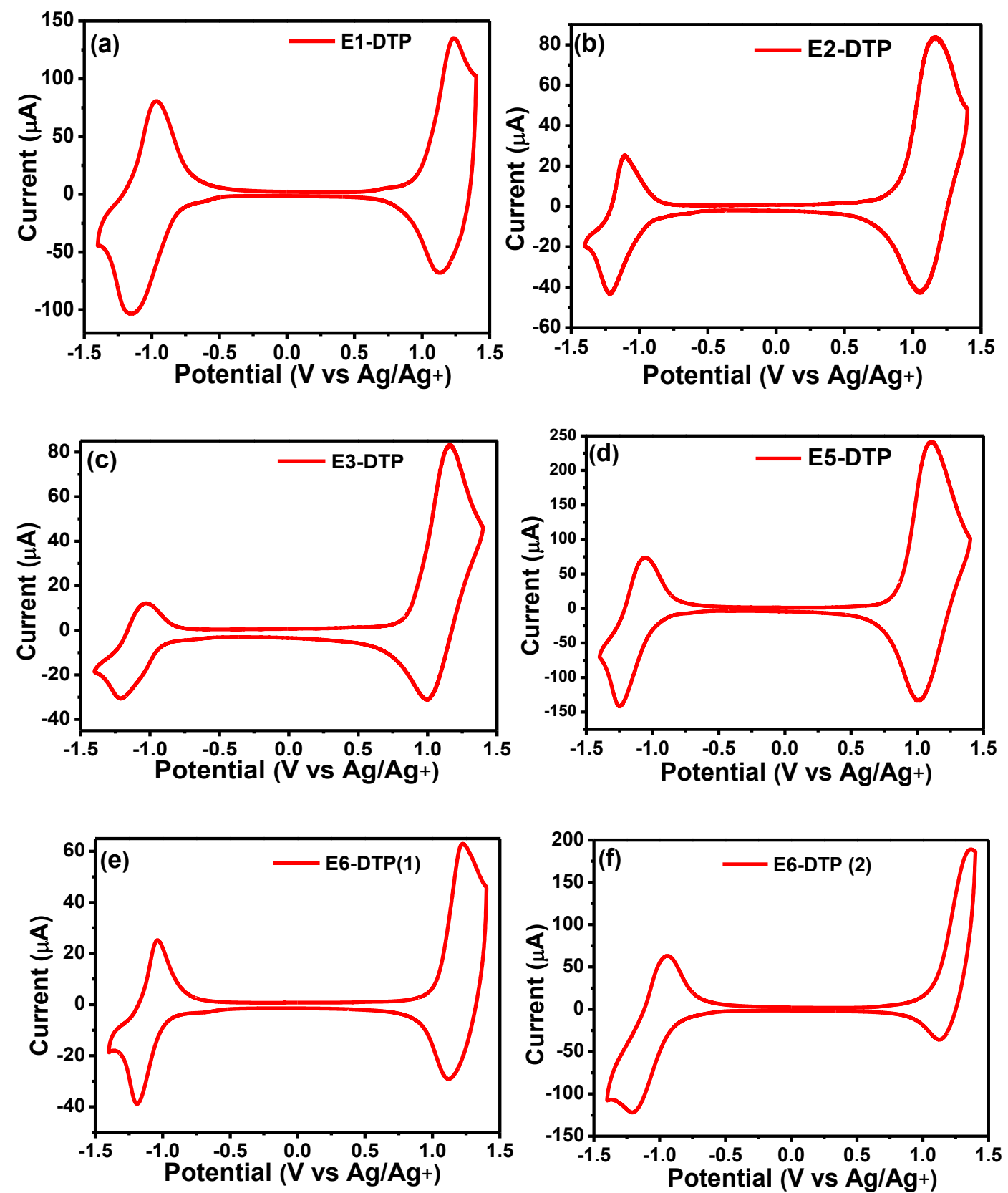


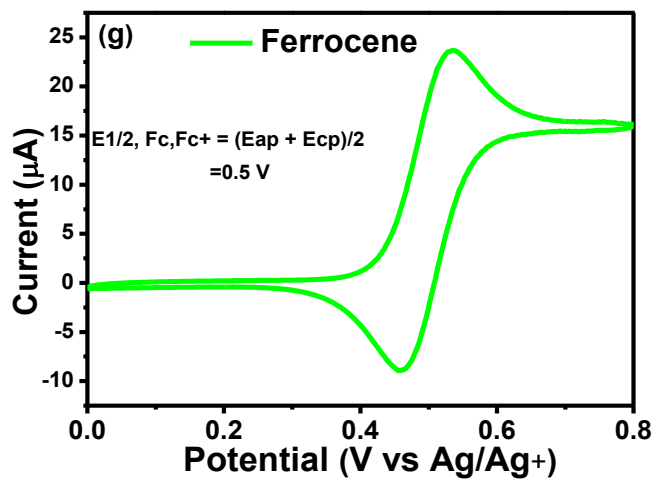

Figure 6.7. Cyclic voltammetry curve for PDFBT-DTP polymers: E1-DTP (a), E2DTP (b), E3-DTP (c), E5-DTP (d), E6-DTP (1) of $\mathrm{CHCl}_{3}$ fraction (e), E6-DTP (2) of chlorobenzene fraction (f) and ferrocene standard (g).

\subsubsection{Field-effect transistor performance}

The charge-transport properties of PDFBT-DTP polymers were studied by fabricating bottom-gate, top-contact OTFT devices on OTS-18 modified $\mathrm{Si} / \mathrm{SiO}_{2}$ substrate. The fabrication and measurement were conducted in the air without any protective atmosphere. The device performance for representative polymer (E2DTP) are summarized in Table 6.4 and their transfer and output characteristics curves are shown in Figure 6.8. The hole mobility $\left(\mu_{\mathrm{h}}\right)$ of the as-prepared OFET devices of E2-DTP polymer, which is derived from the slope of transfer curve, is 1.0 $\times 10^{-4} \mathrm{~cm}^{2} \mathrm{~V}^{-1} \mathrm{~s}^{-1}$. After thermal annealing at $100{ }^{\circ} \mathrm{C}$, a slightly increased $\mu_{\mathrm{h}}$ to $1.7 \times$ $10^{-4} \mathrm{~cm}^{2} \mathrm{~V}^{-1} \mathrm{~s}^{-1}$ was obtained. The morphologies of E2-DTP were investigated by AFM (see Figure 6.9) from its spin cast thin film on OTS-18 treated $\mathrm{Si} / \mathrm{SiO}_{2}$ substrate. At room temperature, E2-DTP films displayed ordered bicontinuous 
networks. However, after annealing at $100{ }^{\circ} \mathrm{C}$, a high degree of nanoscale aggregation was formed, this might explain the increased hole mobility of E2-DTP after annealing.

Table 6.4. Performance characteristics of OFET devices for PDFBT-DTP polymer (E2-DTP) semiconductor on an OTS-18-treatd substrate fabricated and measured ambient conditions.

\begin{tabular}{|l|l|l|l|l|l|}
\hline Entry & $M_{n}$ & Annealing & $\mu_{h}$ & $V_{t h}$ & On/off \\
no. & $(\mathbf{k D a})$ & Temp. $\left({ }^{\circ} \mathbf{C}\right)$ & $\left(\mathbf{c m}^{2} \mathbf{V}^{-1} \mathbf{s}^{-1}\right)$ & $(\mathbf{V})$ & Ratio \\
\hline \multirow{2}{*}{ E2-DTP } & \multirow{2}{*}{17.7} & R.T. & $1.0 \times 10^{-4}$ & 7.6 & $10^{3}-10^{4}$ \\
\cline { 3 - 6 } & & 100 & $1.7 \times 10^{-4}$ & 6.3 & $10^{3}-10^{4}$ \\
\hline
\end{tabular}
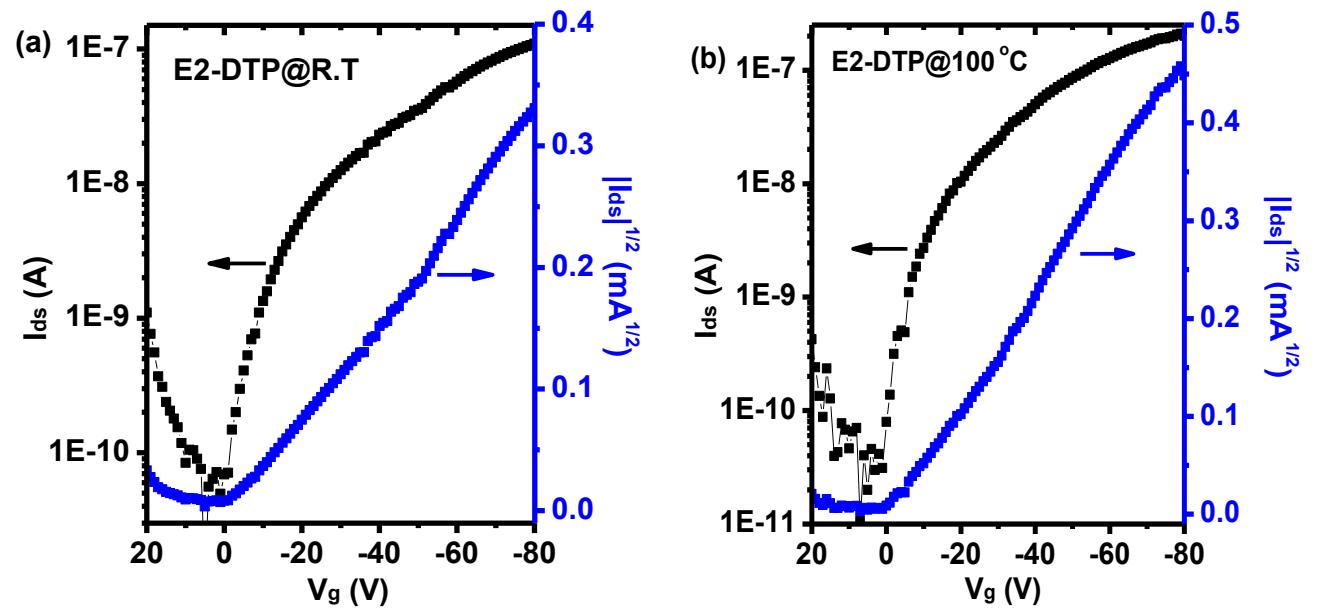


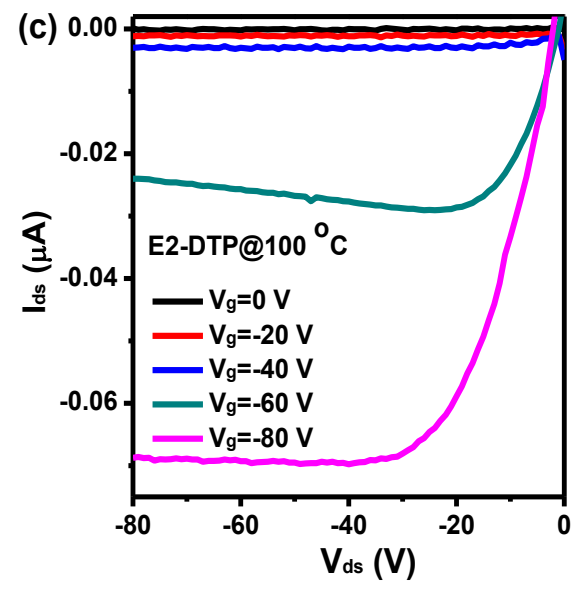

Figure 6.8. Transfer curves of E2-DTP polymer (a) at r.t and (b) after annealing at $100{ }^{\circ} \mathrm{C}$. Output characteristics curve of E2-DTP polymer for after annealing at 100 ${ }^{\circ} \mathrm{C}(\mathrm{c})$.
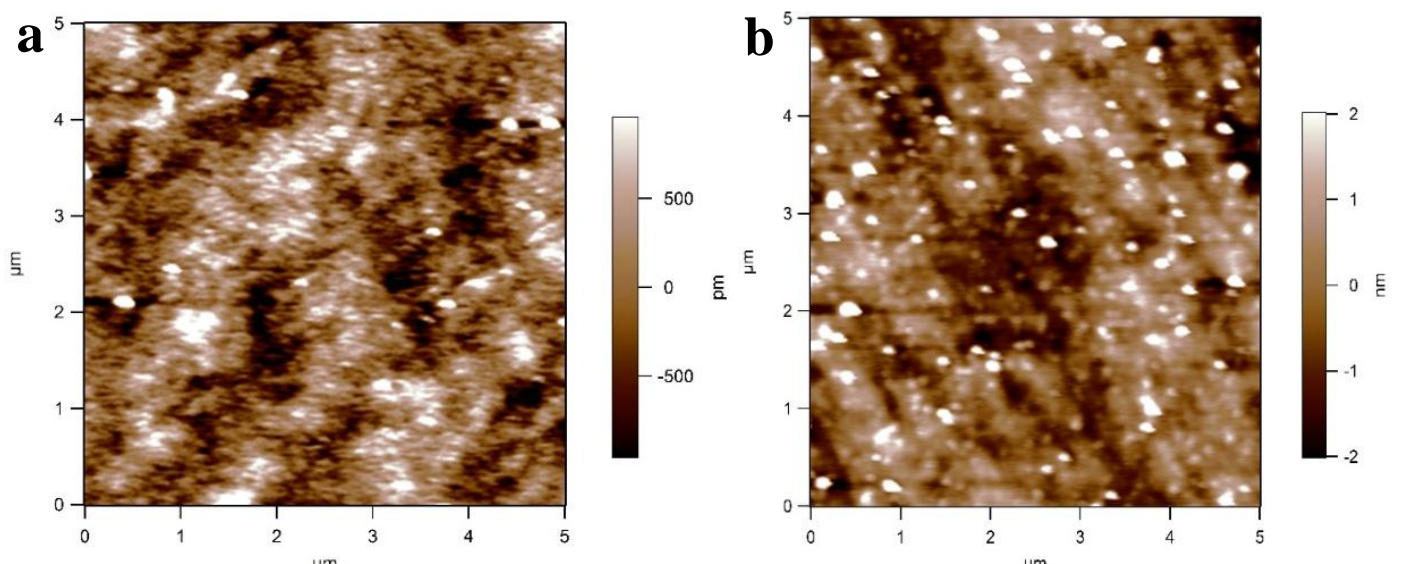

Figure 6.9. AFM height $\left(5 \times 5 \mu \mathrm{m}^{2}\right)$ images for PDFBT-DTP polymer of E2-DTP at R.T. (a) and after annealing at $100{ }^{\circ} \mathrm{C}$ (b). 


\subsection{Conclusion}

In summary, direct arylation polymerization (DAP) has been used in the synthesis of low bandgap 5H-dithieno[3,2-b:2',3'-d]pyran (DTP) based polymer, PDFBT-DTP. A series of DAP optimization reactions have led to PDFBT-DTP with $M_{\mathrm{n}}$ up to $17.7 \mathrm{kDa}$ (Entry E2-DTP) at a catalytic condition of $\mathrm{Pd}_{2}(\mathrm{dba})_{3} /(\mathrm{o}-$ $\mathrm{MeOPh})_{3} \mathrm{P} / \mathrm{PivOH} / \mathrm{K}_{2} \mathrm{CO}_{3} / \mathrm{o}-x y l e n e(0.2 \mathrm{M})$. E2-DTP Entry of PDFBT-DTP has displayed a strong shoulder peak intensity in its absorption spectra, which corresponds to the presence of strong $\pi-\pi$ interaction in a film state. Charge transport behavior of PDFBT-DTP polymers evaluated by OFET device fabricated and measured under ambient condition shows a hole mobility of $1.7 \times 10^{-4} \mathrm{~cm}^{2} \mathrm{~V}^{-1} \mathrm{~s}^{-1}$. These results indicate that further optimization of DAP reaction conditions and OFET device fabrication under inert atmosphere could lead to higher molecular weight and better device performance PDFBT-DTP polymer. 


\section{CHAPTER 7}

\section{Direct Arylation Polymerization towards a Narrow}

\section{Bandgap Conjugated Microporous Polymer with}

\section{Hierarchical Porosity}

\subsection{Introduction}

In chapter 5 and 6 direct arylation polymerization has been used extensively investigated to the synthesis of linear one-dimensional (1D) donor-acceptor conjugated polymers. In this chapter, this synthesis method is extended to another type of multidimensional conjugated polymers, like conjugated microporous polymers. Conjugated microporous polymers (CMPs) are a unique class of polymers that inherently combines $\pi$-conjugation with microporosity. ${ }^{321-327}$ CMPs have attracted increasing interests due to their potential applications in optoelectronics, gas adsorption, storage, separations, and heterogeneous catalysis. ${ }^{328,329}$ To date CMPs have been mainly synthesized through similar methods to linear conjugated polymers, mainly via transition metal catalyzed cross-coupling reactions such as Suzuki coupling, Stille coupling, Sonogashira-Hagihara coupling, and Yamamoto 
coupling. ${ }^{330-334}$ Nevertheless, some of these methods (particularly Stille coupling) generally involve tedious preactivation of $\mathrm{C}-\mathrm{H}$ bonds with organometallic reagents and formation of a stoichiometric amount of toxic byproducts. ${ }^{335,336}$ Cheaper and more efficient synthetic procedures would clearly be a great asset for the large-scale preparation and applications of CMPs.

We have been interested in an emerging polycondensation method called direct arylation polymerization (DAP) as a relatively facile synthetic approach to $\pi$ conjugated polymers. ${ }^{61,283,337-340}$ DAP involves the coupling of an aryl halide monomer directly with another arene monomer without preactivation of the $\mathrm{C}-\mathrm{H}$ bonds. ${ }^{278-280}$ Recently we have employed DAP to synthesize a series of linear donoracceptor (D-A) conjugated polymers and investigated the factors including catalysts, solvents, ligands, bases, additives, the concentration of reactants, and phase transfer agents. Our synthetic optimization of the DAP reactions has led to a variety of highquality polymers with minimal structural defects. ${ }^{83,84,144,151-153}$

DAP has been rarely utilized for the synthesis of CMPs, nevertheless, despite its aforementioned advantages over the other synthetic methods. ${ }^{341}$ This chapter presents the synthesis of a new type of two-dimensional CMPs through DAP of 8,11dibromodithieno[3,2-a:2',3'-c]phenazine (DBDTP) as a single monomer building block (see Scheme 12). While DBDTP has been used as an electron-accepting unit for the synthesis of linear D-A polymers with narrow bandgaps, to the best of our knowledge, it has never been used for the synthesis of CMPs. We expected that C-H 
direct arylation coupling between the dibromo-phenyl group and the $\alpha$-protons in the fused dithiophene of DBDTP may enable the formation of conjugated networks with microporous structures as shown in Scheme 12.

\subsection{Experimental}

Materials. Unless stated otherwise, starting materials and anhydrous solvents were obtained from Sigma-Aldrich and used without further purification. Compound $\mathbf{1 1}^{36}, \mathbf{2 7}^{342,343}$, and DBDTP $(\mathbf{2 8})^{342,343}$ was prepared according reported procedures. All the reactions were carried out under $\mathrm{N}_{2}$ atmosphere.

DFT BLYP 6-31G(d) calculations ${ }^{1}$. Calculations were performed with Gaussian 09 using GaussView 5 to generate structures. Ground state geometry optimizations for both the monomer (DBDTP), Cyclic tetramer $\left(\mathbf{C}_{4}\right)$, Linear tetramer (L4), and Cyclic polymer (C9) model structures were performed at the B3LYP level of theory with the $6-31+\mathrm{G}(\mathrm{d})$ basis set to calculate the free energy per monomer.

- Sum of electronic and thermal free energies at $140{ }^{\circ} \mathrm{C}$ (in Hartrees):

1. Monomer (DBDTP): -6662.525214 Hartree

2. Microporous Polymer (Cyclic-tetramer): -16360.833762 Hartree

3. Linear Polymer (linear-tetramer): -18933.153254 Hartree

4. HBr: -2572.323535 Hartree

- $\quad$ Reaction 1: 4 Monomers $\rightarrow$ Cyclic tetramer $\left(\mathbf{C}_{4}\right)+4 \mathrm{HBr}$

$$
\Delta \mathbf{G}_{1}=-16360.833762+4 *(-2572.323535)-4 *(-6662.525214)
$$




$$
=-0.027046 \mathrm{Hartree}=\mathbf{- 1 6 . 9 7 2} \mathbf{k C a l} / \mathbf{m o l}=\mathbf{- 4 . 2 4 3} \mathrm{kCal} / \mathbf{m o l} \text { per monomer }
$$

- ${ }^{\text {b }}$ Reaction 2: 4 Monomers $\rightarrow$ Linear tetramer $(\mathbf{L} 4)+3 \mathrm{HBr}$

$$
\begin{aligned}
\boldsymbol{\Delta G}_{\mathbf{2}} & =-18933.153254+3 *(-2572.323535)-4 *(-6662.525214) \\
& =-0.023003 \text { Hartree }=\mathbf{- 1 4 . 4 3 5} \mathbf{~} \mathbf{C a l} / \mathbf{m o l}=\mathbf{- 3 . 6 0 9} \mathbf{~} \mathbf{C a l} / \mathbf{m o l} \text { per monomer }
\end{aligned}
$$

- $\quad$ Reaction 3: 9 Monomers $\rightarrow$ Cyclic polymer $(\mathbf{C 9})+12 \mathrm{HBr}$

$$
\begin{aligned}
& \Delta \mathbf{G} 3=-29094.921870+12 *(-2572.323535)-9 *(-6662.525214) \\
& =-0.077364 \mathrm{Hartree}=\mathbf{- 4 8 . 5 4 6 6} \mathbf{k C a l} / \mathbf{m o l}=\mathbf{- 5 . 3 9 4} \mathbf{k C a l} / \mathbf{m o l} \text { per } \text { monomer }
\end{aligned}
$$

\subsubsection{Synthetic monomers}

\section{3,6-dibromobenzene-1,2-diamine $(27)^{342,343}$}

To $150 \mathrm{~mL}$ round bottomed flask containing stir bar 4,7-dibromo benzo[c][1,2,5]thiadiazole (DBrBT) $(2 \mathrm{~g}, 6.8 \mathrm{mmol})$ was dissolved in $60 \mathrm{~mL}$ of anhydrous ethanol under nitrogen atmosphere and then cooled to $0{ }^{\circ} \mathrm{C}$ with ice bath followed by addition of $\mathrm{NaBH}_{4}$ in portion. The reaction mixture was stirred for $6 \mathrm{~h}$ at room temperature and then poured into a flask containing water followed by extraction with diethyl ether $(3 \times)$, the combined organic phase was washed with brine $(2 \times)$, and drying over anhydrous $\mathrm{Na}_{2} \mathrm{SO}_{4}$. The white solid were collected by filtration and used without further purification $(1.38 \mathrm{~g}, 76 \%)$. 


\section{8,11-dibromodithieno[3,2-a:2',3'-c]phenazine, DBDTP (28) ${ }^{342,343}$}

To a $150 \mathrm{~mL}$ round bottomed flask containing stir bar diamine, Compounds 27 (1.38 g, $5.15 \mathrm{mmol})$ and diketone, Compound $11(1.13 \mathrm{~g}, 5.15 \mathrm{mmol})$ were dissolved in ethanol $(60 \mathrm{~mL})$ and acetic acid $(20 \mathrm{~mL})$ under a nitrogen atmosphere. After refluxing for $48 \mathrm{~h}$, an orange precipitate was collected by filtration and washed with cold ethanol. Further purification via recrystallization in chloroform gave an orange solid (2 g, $86 \%)$.

${ }^{1} \mathrm{H}$ NMR $\left(300 \mathrm{MHz}, \mathrm{CDCl}_{3}, \delta\right) 8.56(\mathrm{~d}, \mathrm{~J}=3.12 \mathrm{~Hz}, 2 \mathrm{H}), 8.06(\mathrm{~s}, 2 \mathrm{H}), 7.64$ (d, $\mathrm{J}=3.18 \mathrm{~Hz}, 2 \mathrm{H})$.

\subsubsection{General synthetic protocol for dithieno[3,2-a:2',3' c] phenazine based conjugated microporous organic polymer (DTP-CMP)}

In a glove box, 8,11-dibromodithieno[3,2-a:2',3'-c]phenazine (135 mg, 0.3 mmol), $\mathrm{Pd}_{2}(\mathrm{dba})_{3}(7 \mathrm{mg}, 5 \% \mathrm{mmol}),(\mathrm{o}-\mathrm{MeOPh})_{3} \mathrm{P}(6 \mathrm{mg}, 10 \% \mathrm{mmol}), \mathrm{K}_{2} \mathrm{CO}_{3}(104$ mg, $0.75 \mathrm{mmol}, 5 \mathrm{eq})$, PivOH (8 mg, $0.5 \mathrm{eq})$ and solvent o-xylene (1.5 mL) were added in a microwave reaction vial with a magnetic stirring bar. The vial was sealed with a Teflon-lined cap and then removed from the glove box. The vial was heated in oil bath at different reaction temperature and time to afford a black precipitate, which was isolated by filtration followed by washing continuously with methanol, acetone, DMF, and acetone sequentially followed by Soxhlet washing with 
methanol, chloroform, and THF each for $24 \mathrm{~h}$. The black solid was subjected to overnight drying in a vacuum at $60^{\circ} \mathrm{C}$.

CMP-NTU1 at $100{ }^{\circ} \mathrm{C}$ for $24 \mathrm{~h}$, Yield:110 mg, Anal.Calcd for $\mathrm{C}_{16} \mathrm{H}_{4} \mathrm{~N}_{2} \mathrm{~S}_{2}$ : C, 65.73; H, 2.76; N, 9.58; S, 21.93. Found: C, 52.24; H, 1.08; N, 6.85; S, 16.00.

CMP-NTU2 at $100{ }^{\circ} \mathrm{C}$ for $48 \mathrm{~h}$ in, Yield:100 mg, Anal.Calcd for $\mathrm{C}_{16} \mathrm{H}_{4} \mathrm{~N}_{2} \mathrm{~S}_{2}$ :

C, 65.73; H, 2.76; N, 9.58; S, 21.93. Found: C, 51.18; H, 1.06; N, 6.65; S, 15.54 .

CMP-NTU3 at $140{ }^{\circ} \mathrm{C}$ for $48 \mathrm{~h}$ in, Yield:108 mg, Anal.Calcd for $\mathrm{C}_{16} \mathrm{H}_{4} \mathrm{~N}_{2} \mathrm{~S}_{2}$ :

C, 65.73; H, 2.76; N, 9.58; S, 21.93. Found: C, 58.71; H, 1.70; N, 6.57; S, 15.25.

CMP-NTU4 at $140{ }^{\circ} \mathrm{C}$ for 7 days in, Yield:112 mg, Anal.Calcd for $\mathrm{C}_{16} \mathrm{H}_{4} \mathrm{~N}_{2} \mathrm{~S}_{2}$ :

C, 65.73; H, 2.76; N, 9.58; S, 21.93. Found: C, 61.17; H, 1.88; N, 6.86; S, 15.97. 


\subsubsection{NMR Spectra of monomer}

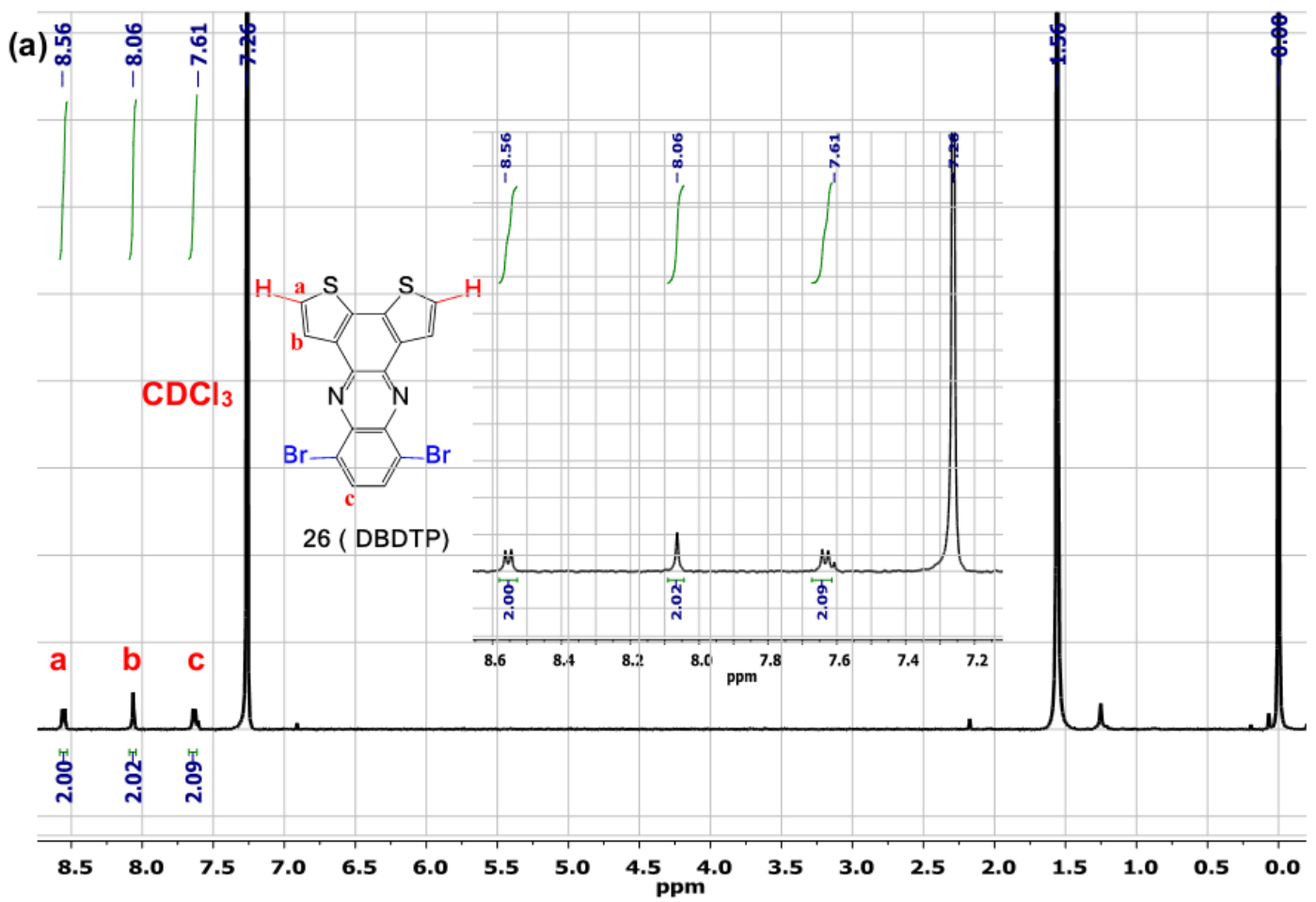

Figure 7.1. ${ }^{1} \mathrm{H} \mathrm{NMR}(300 \mathrm{MHz})$ spectra for compound 26 (a) in a $\mathrm{CDCl}_{3}$ solvent at R.T.

\subsection{Results and Discussion}

\subsubsection{BLYP 6-31G(d) calculations ${ }^{1}$}

The formation of DTP-CMP conjugated polymer networks towards microporous structure is supported by density functional theory (DFT) calculations. The reaction Gibbs free energies $(\Delta \mathrm{G})$ for the formation of a cyclic tetramer (denoted as C4) versus its linear form (denoted as L4) as well as a more extended DTP-CMP network (denoted as C9) were calculated (see Figure 7.2). The $\Delta \mathrm{G}(-4.243 \mathrm{kCal} / \mathrm{mol}$ 
of monomer) for the formation of C4 is slightly more negative than that of L4 (-3.609 $\mathrm{kCal} / \mathrm{mol}$ of monomer). When the conjugated network is extended to $\mathrm{C} 9$, the $\Delta \mathrm{G}$ is even lowered to $-5.394 \mathrm{kCal} / \mathrm{mol}$ of the monomer. These calculation results suggest that the formation of extended cyclic networks as shown in Scheme 12 is thermodynamically favorable. Similar conjugated microporous networks were reported by Cardenas et al. in surface-confined Ullman polymerization of tetrabromotetrathienoanthrathene. ${ }^{344}$

The details of calculation for reaction Gibbs free energies $(\Delta \mathrm{G})$ per monomer for the formation each model structure are described in section 7.2.

\begin{tabular}{|c|c|c|c|}
\hline \multicolumn{2}{|c|}{ Optimized Geometry } & \multirow{2}{*}{$\begin{array}{l}\text { Energy level and bandgap } \\
(\mathrm{eV})\end{array}$} & \multirow[b]{2}{*}{$\begin{array}{l}{ }^{1} \text { Gibbs } \\
\text { free } \\
\text { energy } \\
(\Delta G) \\
\text { (kCal/mol } \\
\text { of the } \\
\text { monomer) }\end{array}$} \\
\hline Top view & Side view & & \\
\hline Monomer - DBDTP & $\frac{8}{85}$ & НОмо = 5.891 & \\
\hline
\end{tabular}




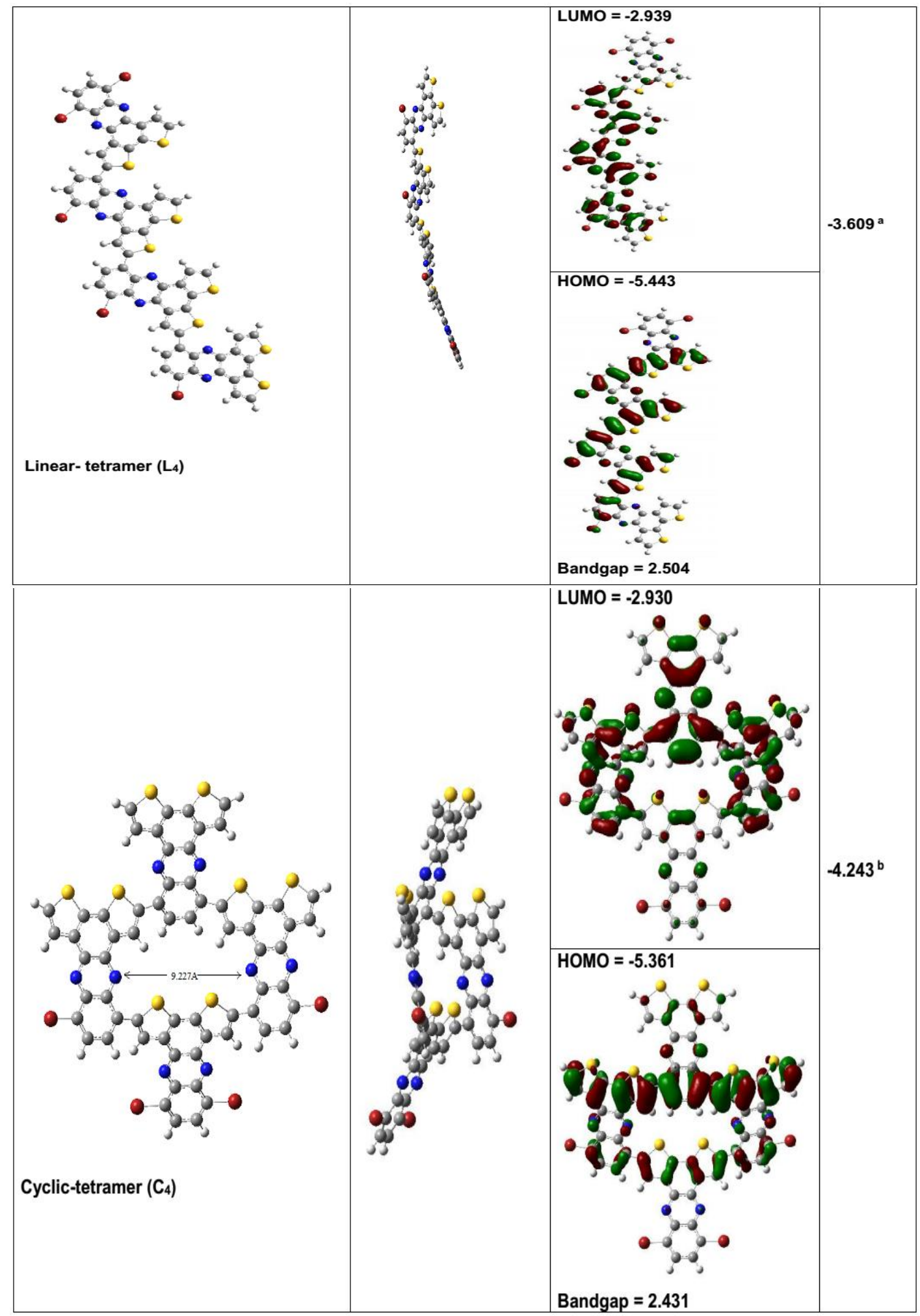




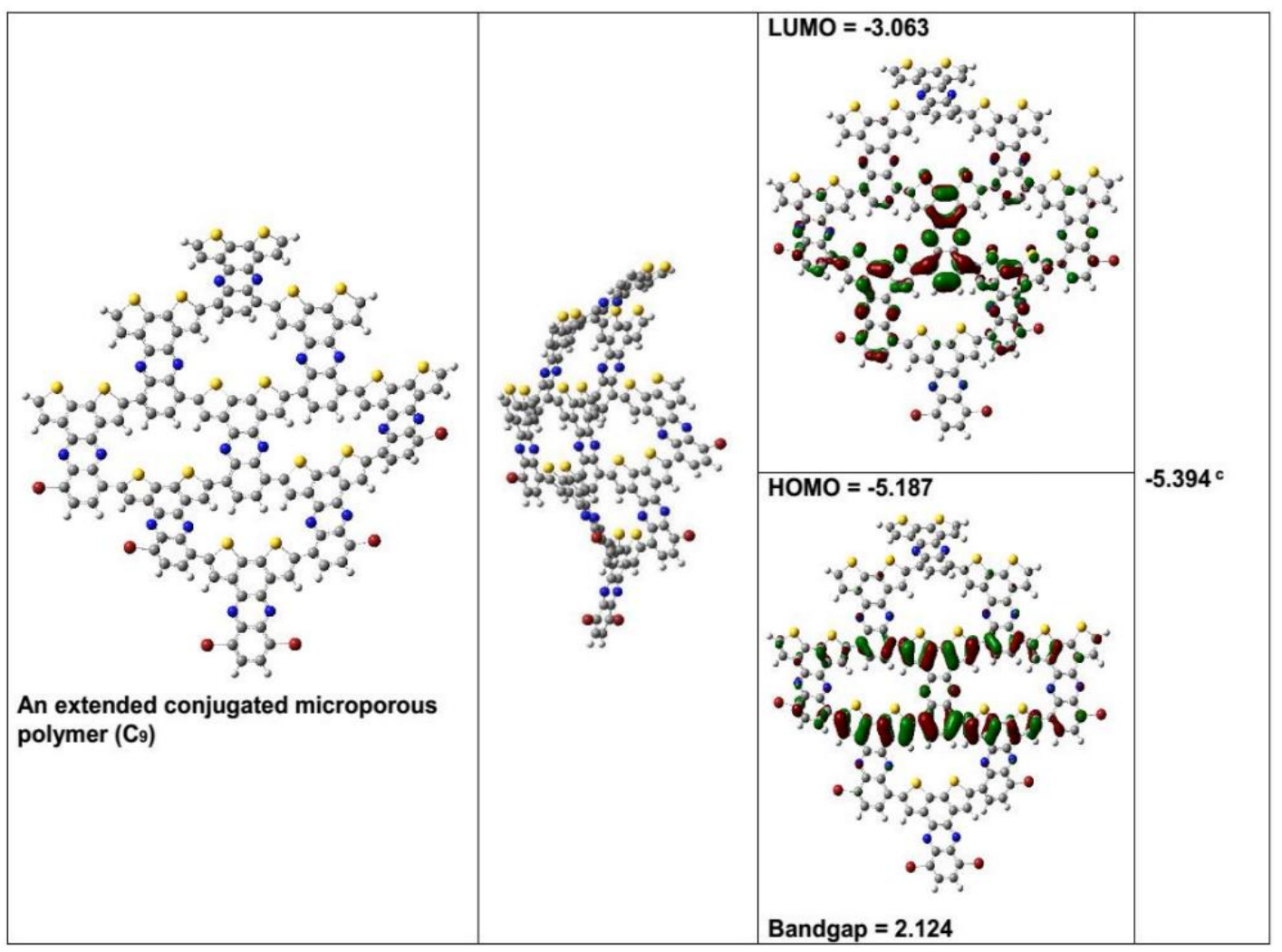

Figure 7.2. Representative geometry-optimized structure by DFT (BLYP 6-31G(d)) for DBDTP monomer, linear tetramer $\left(\mathbf{L}_{4}\right)$, cyclic tetramer $\left(\mathbf{C}_{4}\right)$, and an extended conjugated network $(\mathbf{C 9}){ }^{1}$

\subsubsection{Synthesis of monomers and DTP-CMPs}

The DBDTP monomer (Compound 28) was synthesized based on a previously reported procedure. ${ }^{198,342,343}$ The synthetic routes of the monomer, 8,11dibromodithieno[3,2-a:2',3'-c]phenazine (DBDTP) is demonstrated in Scheme 11. First, 4,7-dibromo-2,1,3-benzothiadiazole was converted to its corresponding 
diamine, (Compound 27) by means of reductive sulfur $(\mathrm{S})$ extrusion reaction with $\mathrm{NaBH}_{4}$. Then, DBDTP was prepared via condensation reaction of diamine (27) with benzo[1,2-b:6,5-b']dithiophene-4,5-dione (11). The DAP polymerization of DBDTP was carried out in the presence of $\mathrm{Pd}_{2}(\mathrm{dba})_{3}(5 \mathrm{~mol} \%)$ mixed with (o-MeOPh $)_{3} \mathrm{P}(10$ mol\%), PiVOH (0.5 equiv), and $\mathrm{K}_{2} \mathrm{CO}_{3}$ (2 equiv) in $o$-xylene. This catalytic condition has been efficient for the synthesis of a series of linear conjugated polymers via DAP. ${ }^{83,84}$ We optimized the synthesis of DTP-CMP polymers by changing the reaction time and temperature to give four batches of DTP-CMP polymers, i.e. CMP-NTU1 $\left(100{ }^{\circ} \mathrm{C}, 24\right.$ h), CMP-NTU2 (100 ${ }^{\circ} \mathrm{C}, 48$ h), CMP-NTU3 $\left(140{ }^{\circ} \mathrm{C}, 48\right.$ h), and CMP-NTU4 (140 ${ }^{\circ} \mathrm{C}, 7$ days).

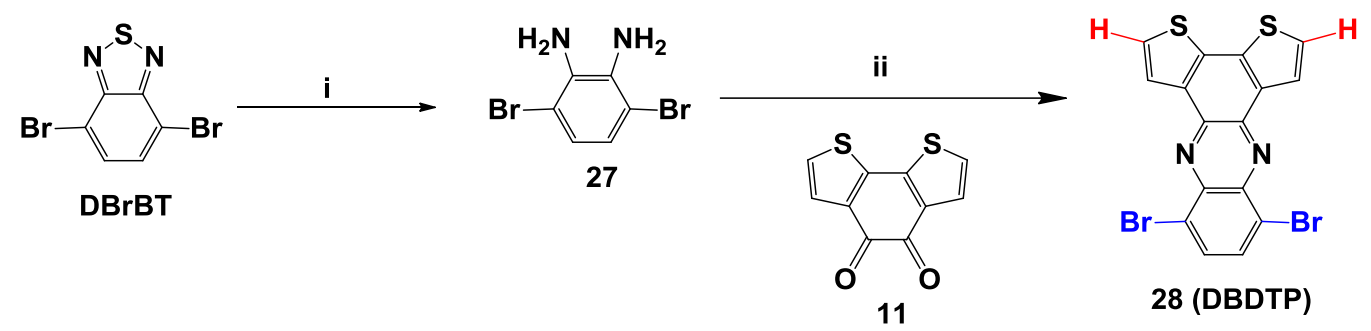

Scheme 11: Synthesis of 8,11-dibromodithieno[3,2-a:2',3'-c]phenazine, DBDTP. (i) NaBH4, EtOH, 6 h, R.T. 76\% yiled (compound 27). (ii) AcOH, EtOH, reflux, 48 h, and $86 \%$ yield (compound $\mathbf{2 8}$ ). 


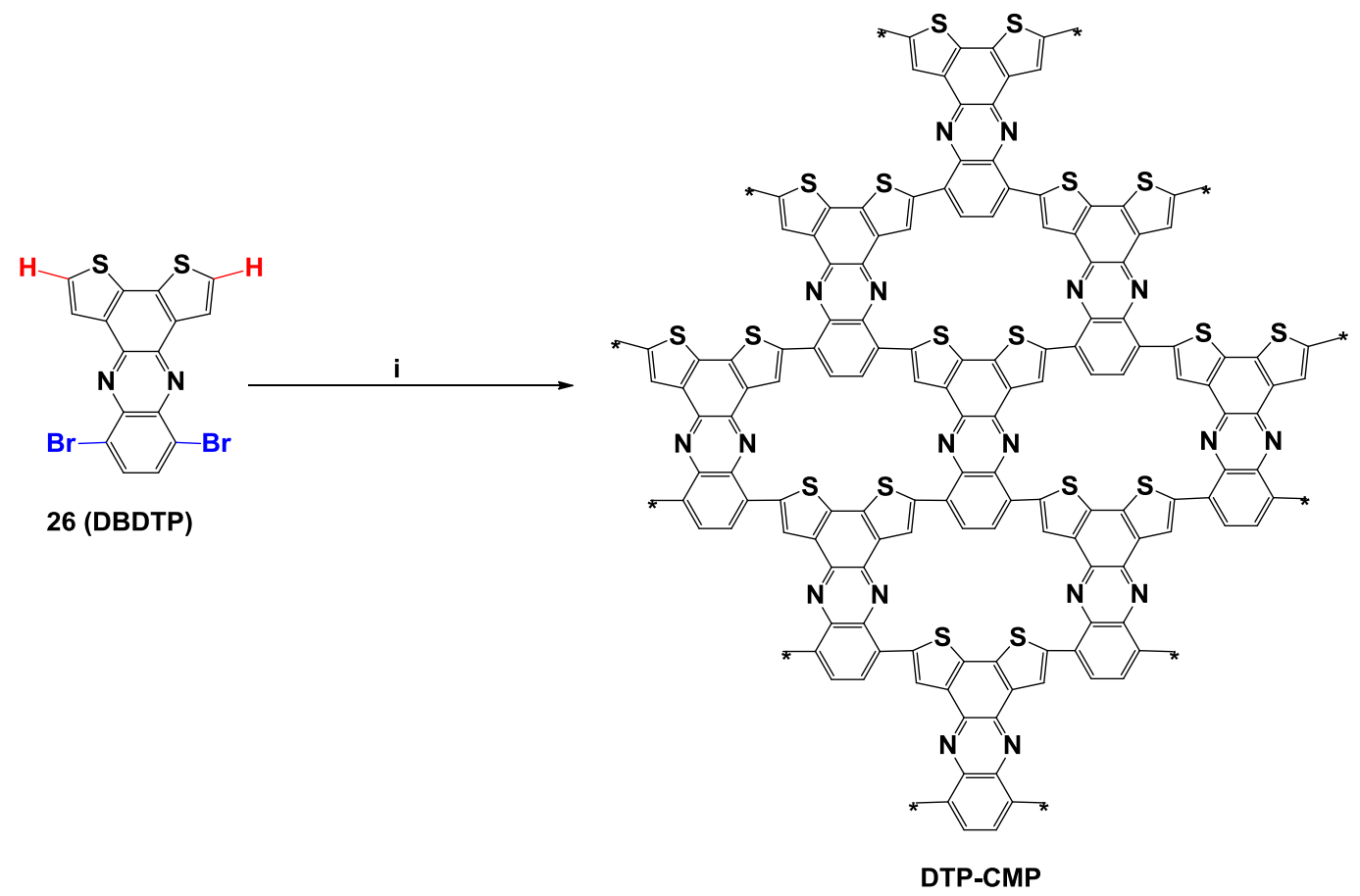

Scheme 12: Synthesis of DTP-CMP via one-step direct arylation polymerization of DBDTP as a single monomer. (i) $\mathrm{Pd}_{2}(\mathrm{dba})_{3}$, (o-MeOPh) ${ }_{3} \mathrm{P}, \mathrm{K}_{2} \mathrm{CO}_{3}, \mathrm{PiVOH}$, o-xylene (see DAP protocol in section 7.2.2).

The obtained DTP-CMP polymers were characterized at the molecular level using Fourier transform infrared spectroscopy (FT-IR). The FT-IR spectra (Figure 7.3 and Table 7.1) shows significant attenuation of the bands at $3000-3100 \mathrm{~cm}^{-1}$ (corresponding to the stretching vibration of $\mathrm{C}-\mathrm{H}$ bonds) and those at 1180 and 580 $\mathrm{cm}^{-1}$ (corresponding to asymmetric and symmetric stretching vibrations of $\mathrm{C}-\mathrm{Br}$ bonds of DBDTP, respectively) in the DTP-CMP polymers compared with the DBDTP monomer. The degree of attenuation increased as the increase of the reaction time to 7 days and the reaction temperature to $140{ }^{\circ} \mathrm{C}$. In contrast, the vibrational 
bands corresponding to $\mathrm{C}=\mathrm{C}$ and $\mathrm{C}=\mathrm{N}, \mathrm{C}-\mathrm{N}$ bonds in the region of $1527-1281,1558$ 1615 and $1054-1135 \mathrm{~cm}^{-1}$, respectively, remain relatively strong intensity in the DTP-CMP polymers but appear broader compared to the monomer. These results provide strong evidence for the polymerization of DBDTP through the $\mathrm{C}-\mathrm{H}$ direct arylation coupling described above.






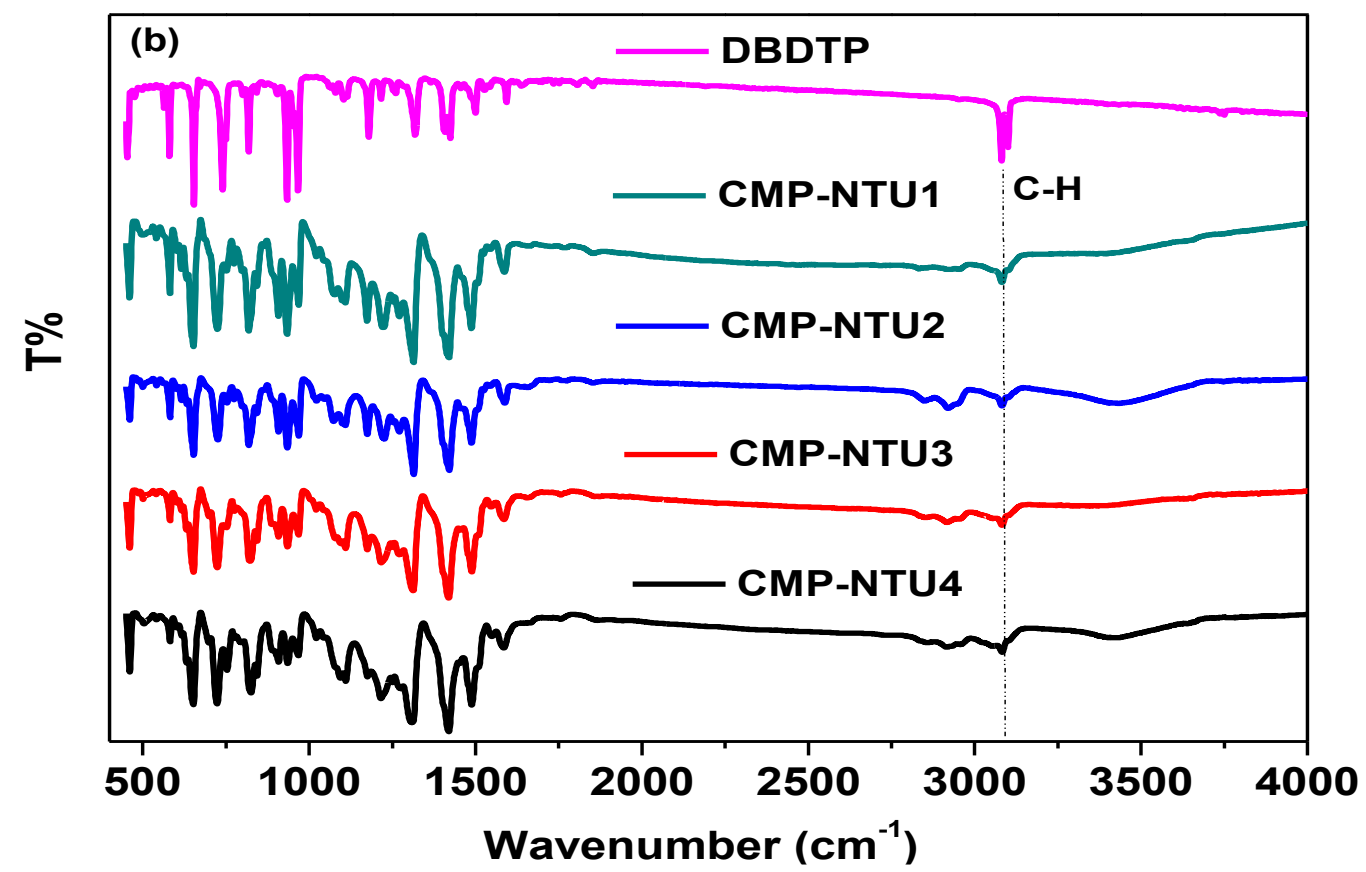

Figure 7.3. FT-IR spectra of DTP-CMPs in comparison to the monomer (DBDTP) (a) zoomed in the range of $400-2200 \mathrm{~cm}^{-1}$ (b).

Table 7.1. Peak assignments for an FT-IR spectrum of DTP-CMP.

\begin{tabular}{|l|l|}
\hline Peak $\left(\mathbf{c m}^{-1}\right)$ & Assignment and Note \\
\hline $\mathbf{3 0 0 0 - 3 1 0 0}$ & Aromatic $\mathrm{C}-\mathrm{H}$ stretch \\
\hline $\mathbf{1 5 5 8 - 1 6 1 5}$ & C=N stretching of the imine \\
\hline $\mathbf{1 4 5 6 - 1 5 2 7}$ & C=C stretch in typical region for fused aromatics \\
\hline $\mathbf{1 3 4 5 - 1 4 6 1}$ & C=C vibrational modes for DBDTP building block \\
\hline $\mathbf{1 2 8 1 - 1 3 4 5}$ & C=C vibrational mode of benzene \\
\hline $\mathbf{1 0 5 4 - 1 1 3 5}$ & C-N stretching \\
\hline $\mathbf{8 0 5 - 8 6 8 , 6 5 1}$ & C-H out of plane bands for p-substituted aromatic \\
\hline $\mathbf{1 1 8 0}$ & Asymmetric $\mathrm{C}-\mathrm{Br}$ \\
\hline $\mathbf{5 8 0}$ & Symmetric $\mathrm{C}-\mathrm{Br}$ \\
\hline
\end{tabular}


Elemental analysis (see Chapter 2 of section 2.7.2) of DTP-CMP polymers showed some discrepancy between the calculated (assuming no bromide end groups) elemental percentages and experimentally found values. Such discrepancy may be caused at least by the following two factors: 1 . The significant contribution of bromide end groups cannot be neglected in the calculation of elemental percentages, particularly when the extent of polymerization is relatively low (e.g. CMP-NTU1); 2. These CMP polymers may not be completely combusted even at a high temperature as shown from the thermogravimetric analysis (TGA) results discussed later. Nevertheless, a better match of the calculated and the measured results of the elemental mass percentages was observed in CMP-NTU4 compared to other batches of the polymers, suggesting its higher degree of polymerization. This result is consistent with the weakest signal of C-Br bonds in CMP-NTU4 as observed in the FT-IR spectra (Figure 7.3).

We took CMP-NTU1 as an example to analyze its chemical structure further by nuclear magnetic resonance (NMR) spectroscopy. The ${ }^{1} \mathrm{H}-\mathrm{NMR}$ spectrum (see Figure 7.4) of the polymer dispersed in $\mathrm{C}_{2} \mathrm{D}_{2} \mathrm{Cl}_{4}$ at $100{ }^{\circ} \mathrm{C}$ showed broad peaks at 7.3-7.1 ppm which correspond to $\beta$-protons from the fused thiophenes of dithienobenenzene group and protons from benzene rings of the phenazine group in the conjugated networks. At the same time, there were three tiny peaks at 8.5, 8.0, and $7.6 \mathrm{ppm}$, respectively, which could be attributed to protons from the periphery groups of the polymers. 


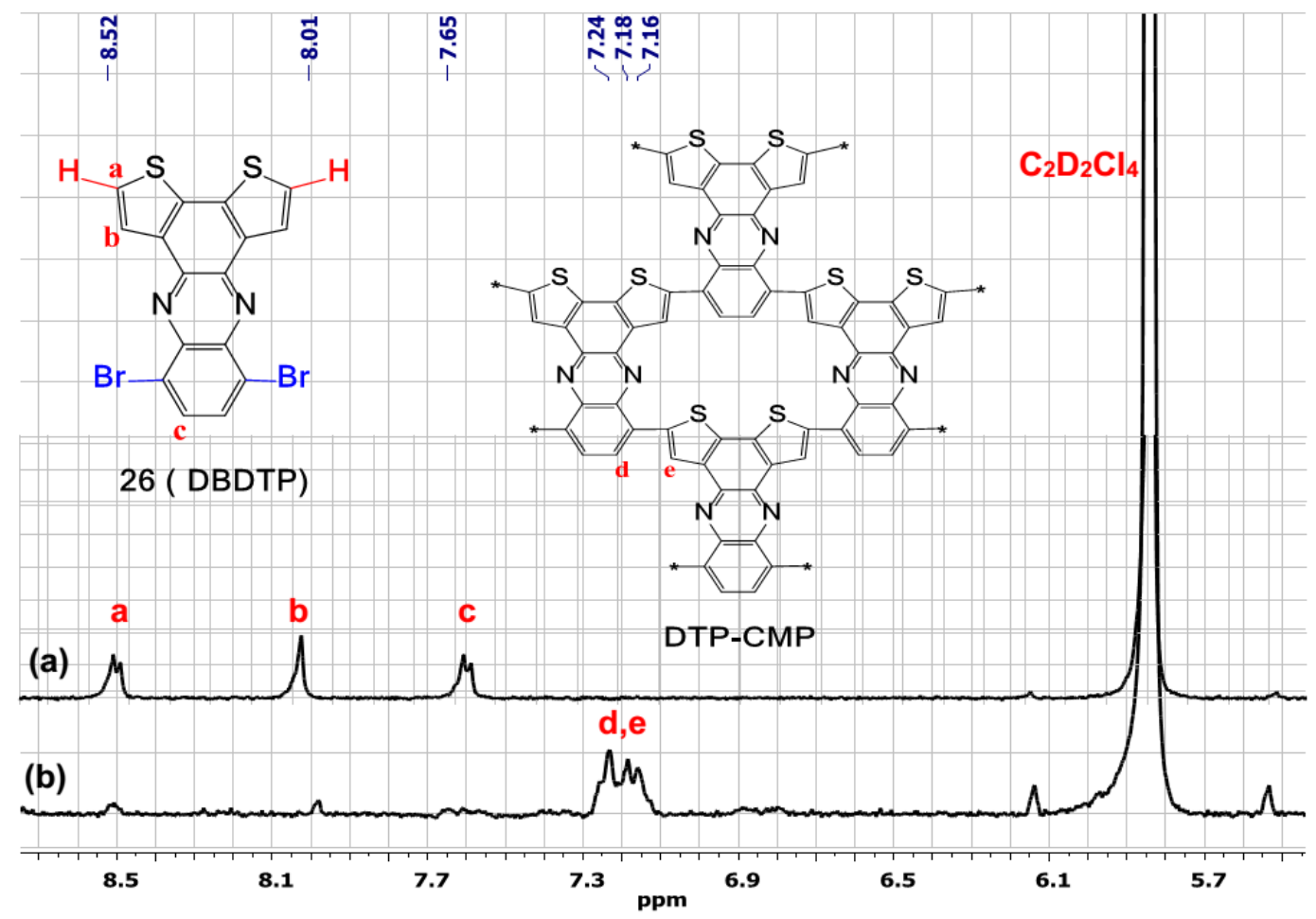

Figure 7.4. ${ }^{1} \mathrm{H}$ NMR spectra (a) DBDTP monomer in $\mathrm{C}_{2} \mathrm{D}_{2} \mathrm{Cl}_{4}$ at r.t., and (b) CMPNTU1 dispersed in $\mathrm{C}_{2} \mathrm{D}_{2} \mathrm{Cl}_{4}$ at $100{ }^{\circ} \mathrm{C}$.

\subsubsection{Thermal properties of DTP-CMPs}

The thermal stability for all batches of DTP-CMP polymers was investigated using thermogravimetric analysis (TGA) at a heating rate of $10{ }^{\circ} \mathrm{C} \bullet \mathrm{min}^{-1}$ under a nitrogen atmosphere. Figure 7.5 shows TGA traces where the onset decomposition temperature $\left(T_{\mathrm{d}}\right)$ with $5 \%$ weight loss above $300{ }^{\circ} \mathrm{C}$, indicating excellent thermal stability of the DTP-CMP polymers. 


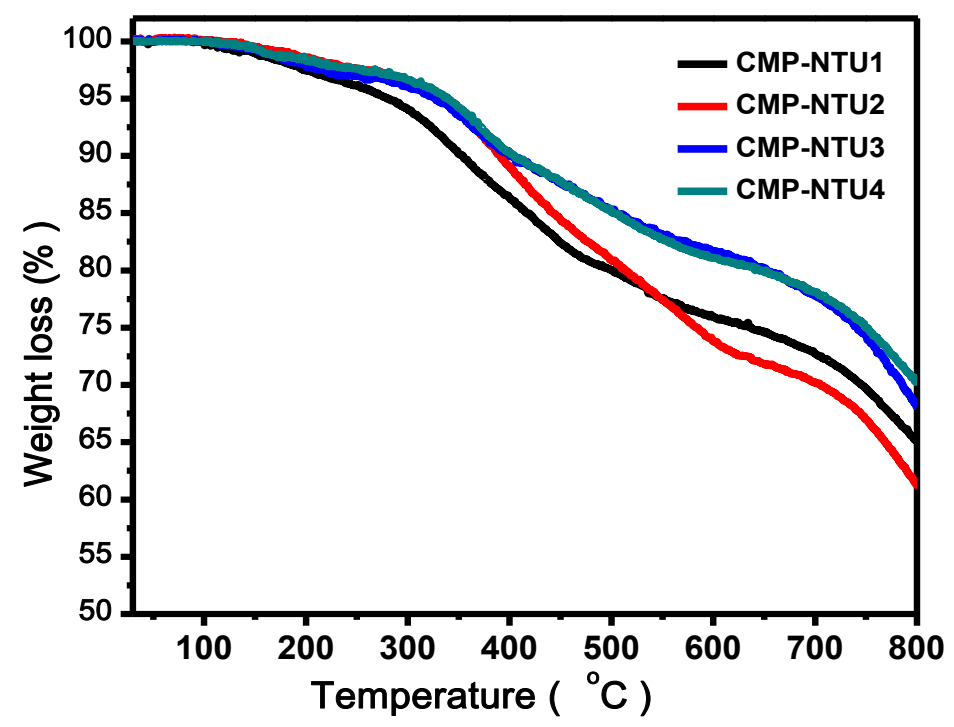

Figure 7.5. TGA plots of DTP-CMP polymers with a heating rate of $10{ }^{\circ} \mathrm{C} \mathrm{min}-1$ under nitrogen.

\subsubsection{Optical and electrochemical properties}

Since DTP-CMP polymers are insoluble in any organic solvents, a suspension was prepared by sonicating a mixture of the corresponding DTP-CMP polymers in chloroform. This resulted in homogeneous translucent dispersion that could be characterized further with UV-vis-NIR absorption spectroscopy. The spectra shown in Figure 7.6 indicate two absorption bands with maximum absorbance $\left(\lambda_{\max }\right)$ at 410 and $610 \mathrm{~nm}$, which corresponds to the localized $\pi-\pi^{*}$ transition and intramolecular charge transfer (ICT) across the conjugated framework, respectively. In comparison to the absorption spectrum of the monomer, a large bathochromic shift of ca. $213 \mathrm{~nm}$ was observed in all batches of the DTP-CMP polymers. This significant shift 
indicates the effective enlargement of the $\pi$-conjugation after DAP reaction. The experimental results are consistent with the DFT calculation (Figure 7.2) analysis which also shows the decrease of the bandgap upon the extension of the cyclic conjugated network.

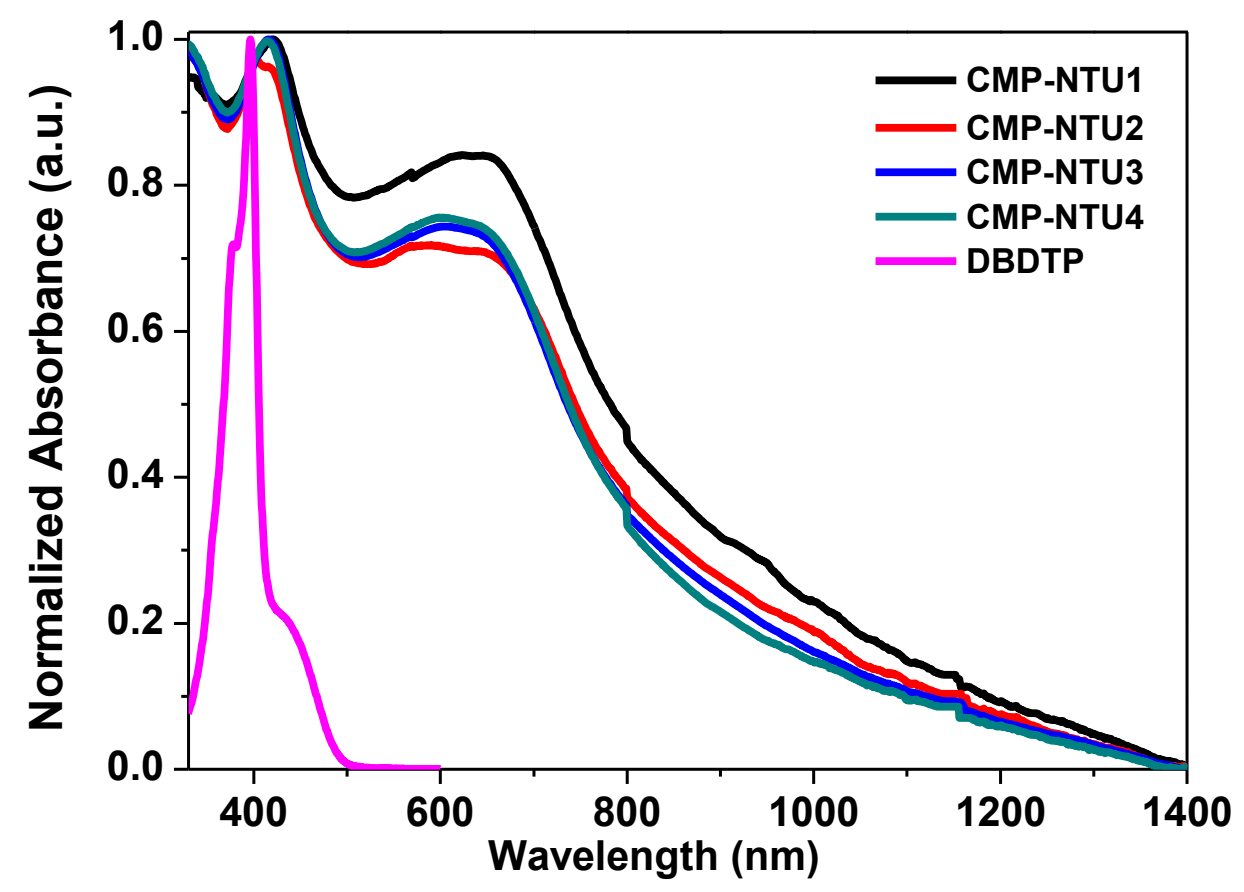

Figure 7.6. UV-vis-NIR absorption spectra of monomer (DBDTP) and DTP-CMP polymers dispersed in $\mathrm{CHCl}_{3}$.

Owing to the light scattering in the dispersion of DTP-CMP polymers, the absorption onset and therefore the optical band gap cannot be determined properly. As a consequence, the electrochemical band gaps (1.4-1.5 eV) of these DTP-CMP polymers were determined from the cyclic voltammograms (Figure 7.7) of thin films 
pasted on glassy carbon as the working electrode, where quasi-reversible oxidation and reduction cycles were observed. The redox properties of DTP-CMP polymers are summarized in Table 7.2.

Table 7.2. Electrochemical properties of DTP-CMP polymers.

\begin{tabular}{|c|c|c|c|c|c|}
\hline DTP-CMP & $\begin{array}{l}\text { Eox }^{\text {onset }} \\
\text { (V) }\end{array}$ & $\begin{array}{l}\text { E }_{\text {red }}^{\text {onset }} \\
\text { (V) }\end{array}$ & $\begin{array}{l}\text { aHOMO } \\
(\mathrm{eV})\end{array}$ & $\begin{array}{l}\left.{ }^{\text {a }}{ }^{(e V)}\right) \\
\text { (elc }\end{array}$ & $\begin{array}{l}{ }^{b} \mathbf{E}_{\mathrm{g}} \text { elc } \\
(\mathbf{e V})\end{array}$ \\
\hline CMP-NTU1 & 1.2 & -0.3 & -5.4 & -3.9 & 1.5 \\
\hline CMP-NTU2 & 1.2 & -0.2 & -5.4 & -4.0 & 1.4 \\
\hline CMP-NTU3 & 1.2 & -0.3 & -5.4 & -3.9 & 1.5 \\
\hline CMP-NTU4 & 1.2 & -0.3 & -5.4 & -3.9 & 1.5 \\
\hline
\end{tabular}

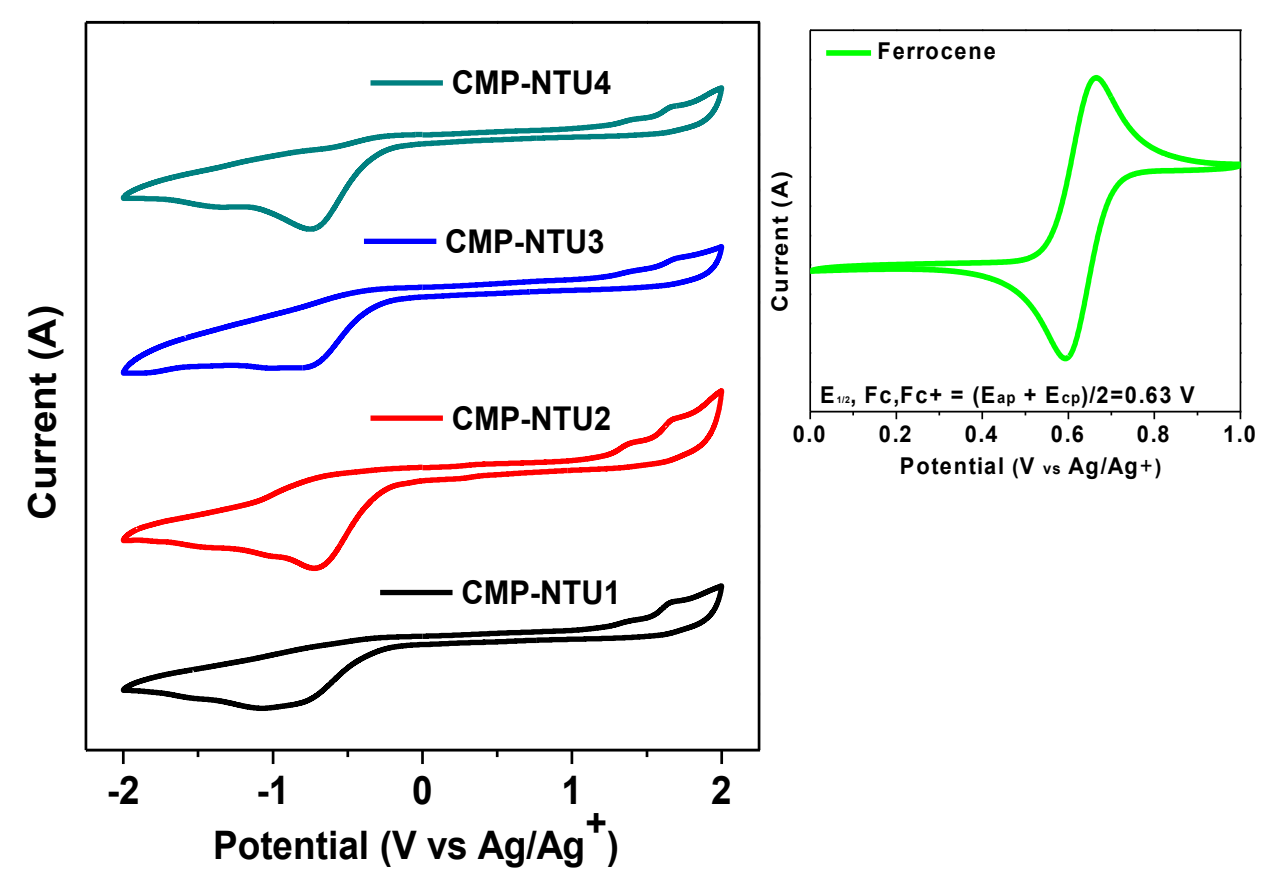

Figure 7.7. Cyclic voltammograms of DTP-CMP polymers. 


\subsubsection{Porosity Characteristics of DTP-CMPs based on Brunauer- Emmett-Teller (BET) measurements}

The porous properties of DTP-CMP polymers were studied by sorption analysis using $\mathrm{N}_{2}$ as sorbate molecule. $\mathrm{N}_{2}$ adsorption-desorption isotherms of DTPCMP polymers measured at $77 \mathrm{~K}$ are shown in Figure 7.8 and the results are summarized in Table 7.3. Prior to porosity measurement, the samples were degassed at $100{ }^{\circ} \mathrm{C}$ under vacuum for $24 \mathrm{~h}$. According to the IUPAC classification, all DTPCMP polymers exhibit a combination of type I \& II nitrogen gas sorption isotherms. ${ }^{345}$ The isotherms show a continuous increase after the adsorption at low relative pressure, indicating an adsorption on the outer surface of small particles. The increase in the nitrogen adsorption at a higher relative pressure above 0.9 may arise in part from interparticulate porosity associated with the meso- and macrostructures of the polymers and interparticular voids. ${ }^{331,346}$ In addition, DTP-CMP polymers shows H3 hysteresis loop, which is an indication for the aggregates of plate-like particles giving rise to slit-shaped pores. Low-pressure hysteresis is also attributed to the presence of microporosity. ${ }^{345,347,348}$ 


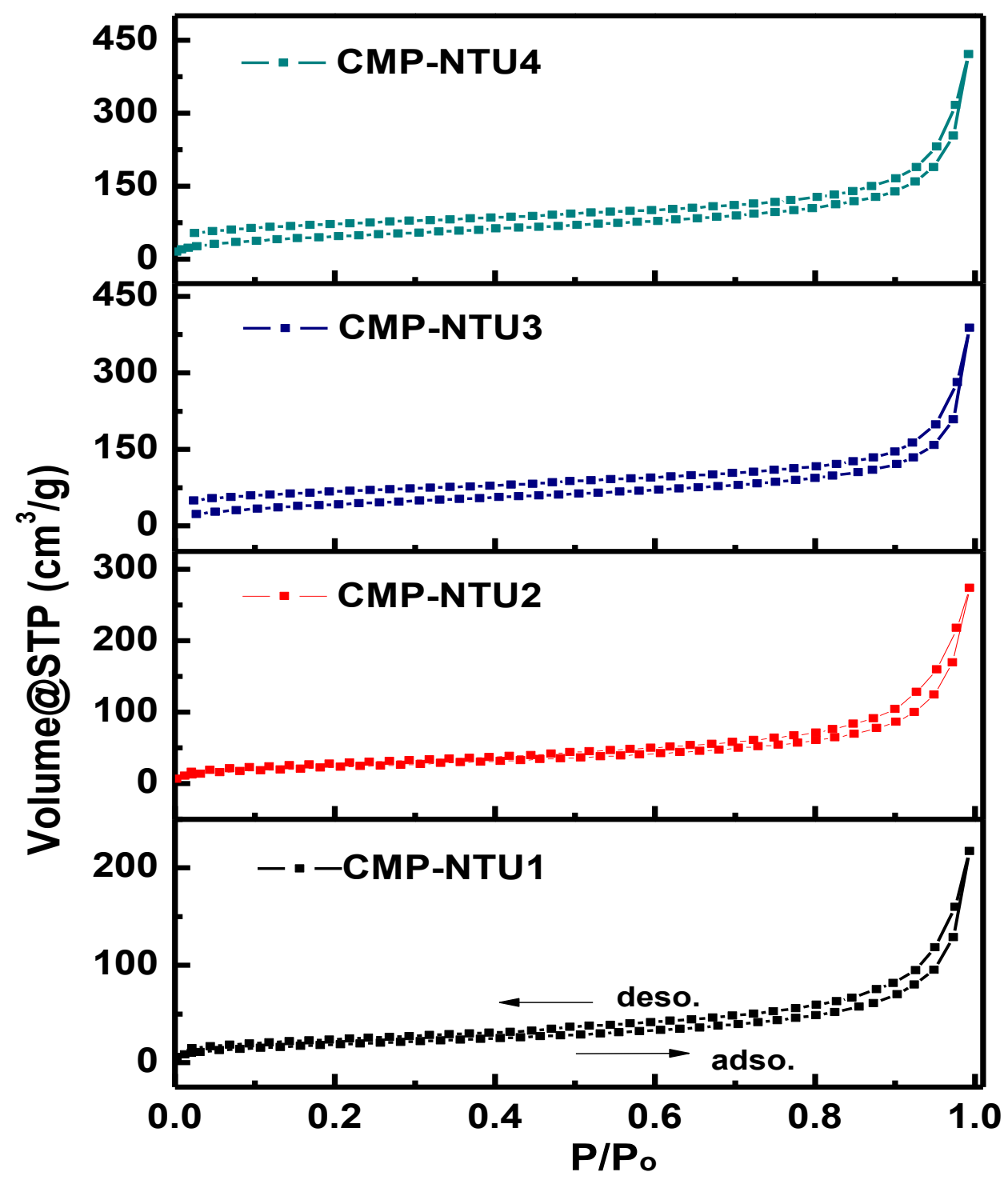

Figure 7.8. Nitrogen gas adsorption-desorption isotherms of DTP-CMP porous polymers measured at $77 \mathrm{~K}$. 
Table 7.3. Porosity properties of DTP-CMP polymers.

\begin{tabular}{|l|l|l|l|}
\hline Entry no. & $\begin{array}{l}\text { BET/Langmuir } \\
\text { surface area }\left(\mathbf{m}^{2} / \mathbf{g}\right)\end{array}$ & $\begin{array}{l}\text { Pore } \\
\text { diameter(㐫) }\end{array}$ & $\begin{array}{l}\text { Pore } \\
\text { volume(cc/g) }\end{array}$ \\
\hline CMP-NTU1 & $70 / 525$ & 8.79 & 0.34 \\
\hline CMP-NTU2 & $87 / 684$ & 8.68 & 0.45 \\
\hline CMP-NTU3 & $160 / 864$ & 17.72 & 0.60 \\
\hline CMP-NTU4 & $175 / 892$ & 12.01 & 0.70 \\
\hline
\end{tabular}

Table 7.3 summarizes the key structural properties derived from the isothermal data such as BET and Langmuir specific surface area, pore diameter and volume. The specific surface area calculated in the relative pressure $\left(\mathrm{P} / \mathrm{P}^{\circ}\right)$ ranging from 0.05 0.25 shows the BET specific surface area of $70,87,160$ and $175 \mathrm{~m}^{2} \mathrm{~g}^{-1}$ for the CMPNTU1, CMP-NTU2, CMP-NTU3, and CMP-NTU4 polymers, respectively. It should be noted that specific surface area of DTP-CMP polymers increases linearly with reaction time and temperature, thereby proving the formation of an extended network of CMP polymers. 


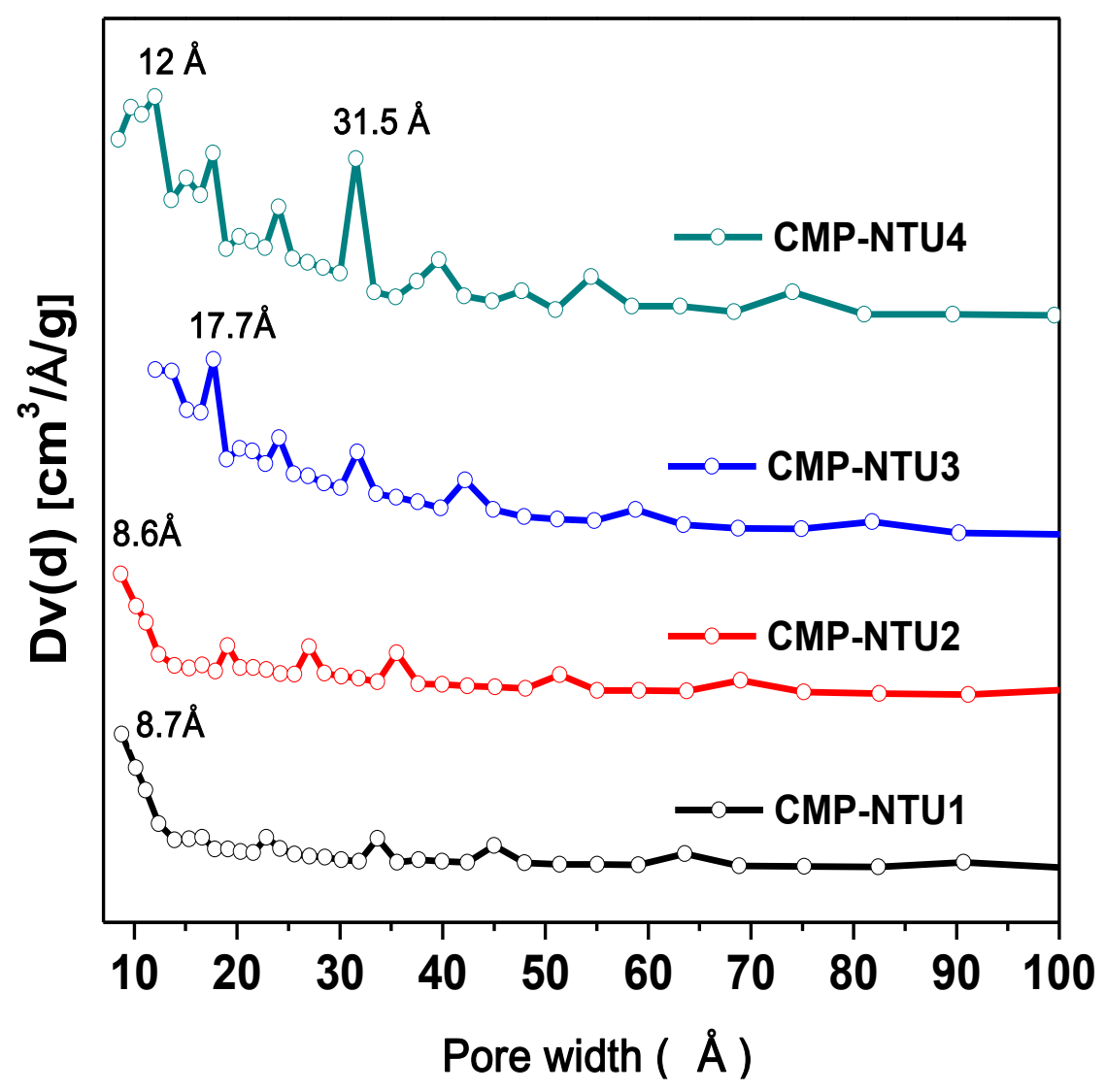

Figure 7.9. Pore size distribution profiles for DTP-CMP porous polymers.

The pore size distribution (PSD) analysis based on the non-local density functional theory (NLDFT) approach has been used extensively to characterize a wide variety of porous materials although it does have limitations. ${ }^{349}$ As shown in Figure 7.9, PSD of DTP-CMP polymers calculated from the adsorption branch of the isotherms with the NLDFT approach indicates a wide PSD ranging from 8 to 40 $\dot{A}$ with dominant pore diameter centered at about 8.79, 8.68, 17.72, $12.01 \dot{\mathrm{A}}$ for CMP-NTU1, CMP-NTU2, CMP-NTU3, and CMP-NTU4 polymers, respectively. 
The measured microporous size distributions are a good match with the pore size estimated by Gaussian 09, DFT calculation (see Figure 7.2 of $\mathbf{C 4}$ ). As shown in Table 7.3

pore diameters and pore volumes show a clear trend similar to the specific surface area as a function of reaction time and temperature. ${ }^{345,349}$

\subsubsection{Morphologies of the DTP-CMP polymers}

The morphologies and internal structures of the DTP-CMP polymers were characterized using scanning electron microscopy (SEM), transmission electron microscopy (TEM) and powder X-ray diffraction (PXRD) spectra. SEM images (see Figure 7.10) of DTP-CMP polymers demonstrate the presence of hierarchical structures of relatively uniform and rigid rods, which aggregate further into largerscale tubes and flat sheets. In addition, as the reaction temperature and time increased to $140{ }^{\circ} \mathrm{C}$ and 7 days, respectively, the density among these rod-like structures increased in CMP-NTU4 compared to other batches of the polymer, resulting in a high density of slit-like pores and higher surface area as observed in the sorption analysis shown in Figure 7.8. TEM images (see Figure 7.11) also support the formation of microporosity and the presence of slit-like mesoporous structures of the

polymers. PXRD spectra (see Figure 7.12) show broad peaks centered at $25^{\circ}$, the characteristics of non-ordered, amorphous structures. 


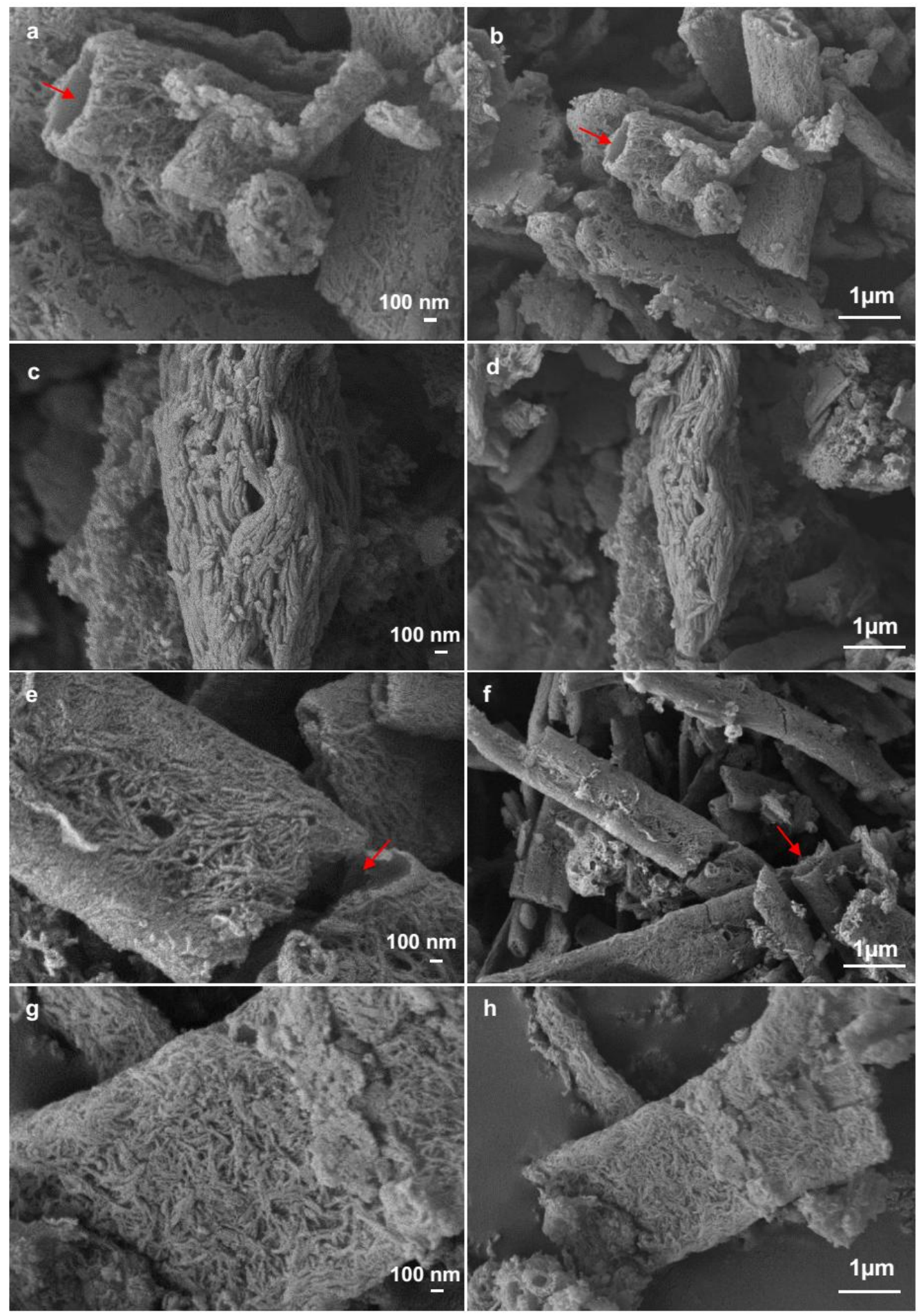

Figure 7.10. Representative SEM images for CMP-NTU1 (a, b), CMP-NTU2 (c, 
d), CMP-NTU3 (e, f), and CMP-NTU4 (g, h). Red arrows indicate the tube-like structures of CMPs.

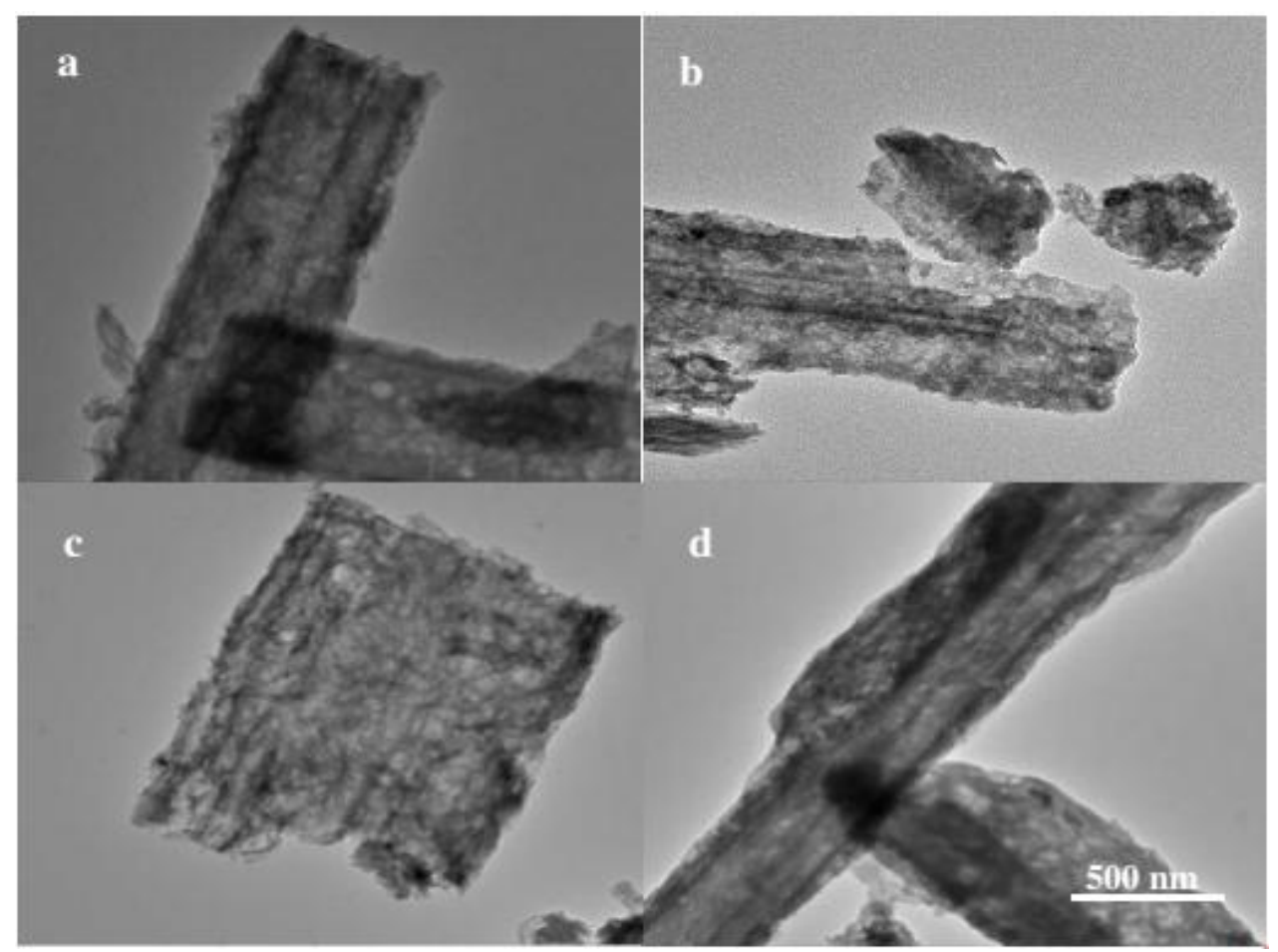

Figure 7.11. TEM images for DTP-CMP for CMP-NTU1 (a), CMP-NTU2 (b), CMP-NTU3 (c), and CMP-NTU4 (d). 


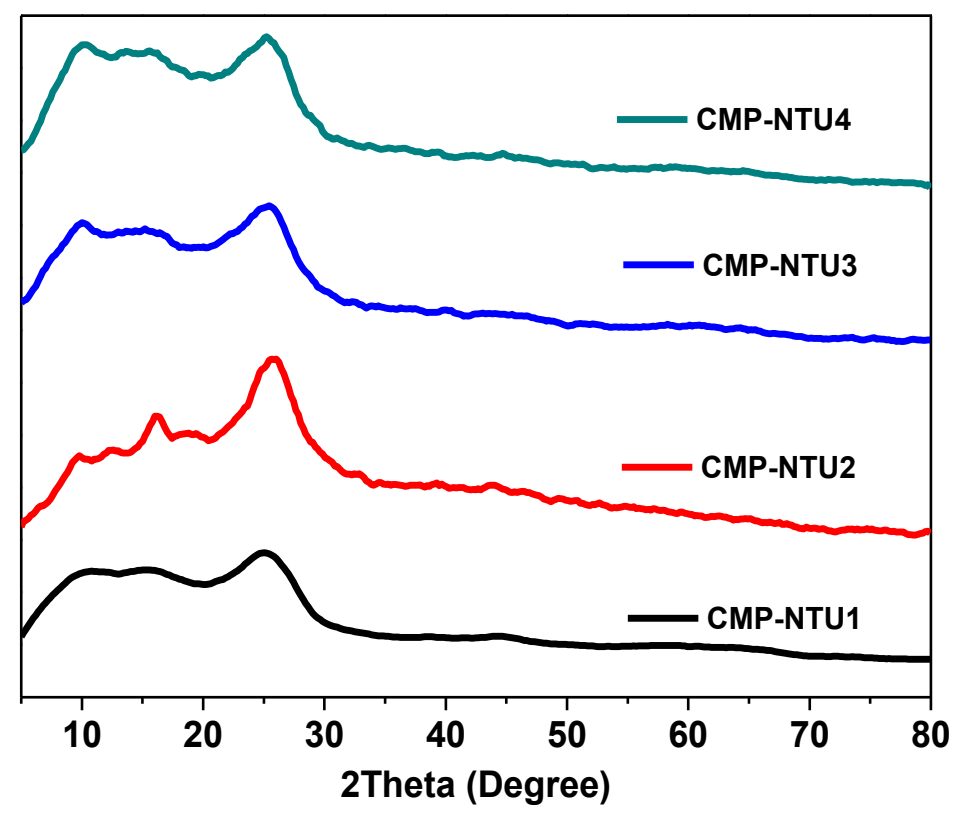

Figure 7.12. PXRD spectra for DTP-CMP.

\subsection{Conclusion}

Narrow-bandgap conjugated microporous polymers have been synthesized via facile direct arylation polymerization of 8,11-dibromodithieno [3,2-a:2',3'c]phenazine. The resulting polymers form hierarchical structures of relatively uniform and rigid nanorods which aggregate further into larger-scale tubes and flat sheets. These polymers displayed good thermal stability, moderate surface area and dominant pore size distribution in the microporous region. The porosity could be tuned by changing the reaction time and temperature of the polymerization. This work demonstrates that $\mathrm{C}-\mathrm{H}$ direct arylation coupling as a facile synthetic tool enables efficient synthesis of conjugated microporous polymers with controllable 
structures and properties. We are currently broadening the scope to other CMPs that can be synthesized via DAP and also explore their applications such as in selective gas adsorption, photocatalysis, and sensing.

\section{The content in Chapter 7 has been published in}

- Polymer Chemistry, 2016 DOI: 10.1039/c6py00719h 


\section{CHAPTER 8}

\section{Summary and Suggestions for Future Work}

\subsection{Summary}

In this dissertation, a novel acceptor building blocks, dithienobenzo chalcogenodiazole and $\gamma$-lactone-Pechmann dye were synthesized and incorporated into the construction of donor-acceptor conjugated polymers via traditional coupling methods (Stille and Suzuki couplings). These D-A conjugated polymers are fully characterized and used as an active layer for applications in the organic electronics devices (OFETs and OPVs). The effect of latent side group and its deprotection method on the physicoelectrochemical properties was investigated by synthesizing tBoC-isoindigo base D-A conjugated polymer. On the other hand, direct $\mathrm{C}-\mathrm{H}$ arylation coupling, which is greener and economical method than traditional coupling methods like (Stille and Suzuki couplings) has been used for the synthesis of 5,6-difluoro-2,1,3-benzothiadiazole (DFBT) based D-A conjugated polymers. A series of optimization reactions has been carried out in order to get high molecular weight polymers. This coupling method is even extended to the synthesis of other

types of conjugated polymer networks, like two-dimensional (2D) conjugated microporous polymers based on a single monomer unit, 8,11-dibromodithieno[3,2- 
a:2',3'-c]phenazine (DBDTP) which bears both aryl bromide and arenes $(\mathrm{C}-\mathrm{H})$ functional groups together were fully characterized.

In Chapter 2, D-A conjugated polymers, P1, P2, P3, PF-S, PF-O, comprising 2,1,3-dithienobenzochalcogenodiazole acceptor were synthesized via Stille and Suzuki polymerizations and used as a semiconducting active layer in OPVs and OFETs devices. All polymers exhibited red shift thin-film absorption spectra accompanied with the appearance of shoulder peaks, indicating the formation of more planar polymer backbone orderings or some $J$-aggregates in their solid states. UV-vis and CV studies revealed that oxadiazole-containing polymers displayed deeper LUMO levels and more red-shifted thin-film absorption spectra when compared to their respective thiadiazole-containing counterparts. Among all the polymers, $\mathbf{P 2}$ based OPVs and OFETs gave the highest PCE of over $2 \%$ and hole mobility of $2.6 \times 10^{-2} \mathrm{~cm}^{2} \mathrm{~V}^{1} \mathrm{~s}^{1}$. The electronic device performance could be further improved through structural and synthesis optimization.

In Chapter 3, D-A conjugated polymers $\mathbf{P 4}$ and $\mathbf{P 5}$ were synthesized via Stille polymerization based on a novel electron acceptor moiety, $\gamma$-lactone-Pechmann dye alternating with 2D-benzo[1,2-b:4,5- $\left.b^{\prime}\right]$ dithiophene (2D-BDT) and alkoxybenzo[1,2-b:4,5-b']dithiophene (1D-BDT) electron donor moiety. DFT calculation indicates that $\gamma$-lactone-Pechmann dye exhibit higher degree of coplanarity and stronger electron-accepting properties than its lactam analogs. The P5 and P6 polymers exhibit a broad absorption band extended to $1000 \mathrm{~nm}$ and narrow band gap 
with deeper LUMO energy level. The thin film field-effect transistors of P5 and P6 showed p-type and ambipolar semiconducting properties, respectively. These results show that $\gamma$-lactone-Pechmann dye could be used as the potential electron-accepting building block for the construction of new conjugated D-A polymers.

In chapter 4, D-A conjugated polymer comprising tert-butoxycarbonyl (t-Boc) substituted isoindigo acceptor moiety was designed, synthesized, and characterized. The impact of t-BoC latent side group deprotection method (chemical vs. thermal) is compared based on the physicochemical characterizations. Therefore, removing tBoC side group via chemical method (treatment with $\mathrm{CF}_{3} \mathrm{COOH}$ acid) gives less redshifted absorption spectra as compared with the thermal treatment method of its spincast thin film. In addition to this, removing the $\mathrm{t}-\mathrm{BoC}$ group has greatly reduced the solubility of the polymers and hinder its spin cast processing. Therefore, OFETs based on the thermally treated thin film of the polymer has given a hole mobility of $9.7 \times 10^{-5} \mathrm{~cm}^{2} \mathrm{~V}^{-1} \mathrm{~s}^{-1}$.

In Chapter 5, direct arylation polymerization (DAP) has been used in the synthesis of 5,6-difluoro-2,1,3-benzothiadiazole (DFBT) based polymers, PDFBTTh4. A series of DAP reactions optimization has led to a best catalytic condition of $\mathrm{Pd}(\mathrm{OAc})_{2} /(\mathrm{o}-\mathrm{MeOPh})_{3} \mathrm{P} / \mathrm{PivOH} / \mathrm{K}_{2} \mathrm{CO}_{3} /$ o-xylene to give the high molecular weight of PDFBT-Th4 polymer with $M_{\mathrm{n}}$ up to $27 \mathrm{kDa}$. The PDFBT-Th4 4 polymer has displayed a strong interchain aggregation behavior in a solid thin film state, this phenomenon gets intensified after annealing. PDFBT-Th4 Polymer has displayed a 
comparable FET charge transport behavior and it gets higher after annealing which is in agreement with UV-vis and AFM results. This Chapter represents a key step further to broadening the scope of high-quality semiconducting polymers that can be synthesized via DAP.

In Chapter 6, direct arylation polymerization (DAP) has been used in the synthesis of low bandgap PDFBT-DTP polymers based on a strong donor moiety, 5H-dithieno[3,2-b:2',3'-d]pyran (DTP) and 5,6-difluoro-2,1,3-benzothiadiazole (DFBT) as an acceptor . A series of DAP optimization has led to a best catalytic condition of, $\mathrm{Pd}_{2}(\mathrm{dba})_{3} /(\mathrm{o}-\mathrm{MeOPh})_{3} \mathrm{P} / \mathrm{PivOH} / \mathrm{K}_{2} \mathrm{CO}_{3} /$ o-xylene $(0.2 \mathrm{M})$ with $M_{\mathrm{n}}$ up to $17.7 \mathrm{kDa}$. Charge transport behavior of PDFBT-DTP polymers evaluated by OFET devices fabricated and measured at ambient condition shows a hole mobility of $1.7 \times 10^{-4} \mathrm{~cm}^{2} \mathrm{~V}^{-1} \mathrm{~s}^{-1}$. These results indicate that further optimization of DAP reaction conditions and fabrication of OFET devices under inert atmosphere could lead to high molecular weight and good performance PDFBT-DTP polymer.

In Chapter 7, direct arylation polymerization (DAP) has been further extended in the synthesis of other types of materials like conjugated polymer networks (conjugated microporous polymers, CMPs). Narrow-bandgap conjugated microporous polymers have been synthesized via facile direct arylation polymerization of 8,11-dibromodithieno [3,2-a:2',3'-c]phenazine. The DAP reactions optimization has been done by changing the reaction time and temperatures 
independently, therefore, it was possible to tune the porosity parameters of the CMPs. The resulting polymers form hierarchical structures of relatively uniform and rigid nanorods which further aggregate into larger-scale tubes and flat sheets. This work demonstrates that $\mathrm{C}-\mathrm{H}$ direct arylation coupling as a facile synthetic tool enables efficient synthesis of conjugated microporous polymers with controllable structures and properties. This work could be used as a benchmark work for the future synthesis of other types of CMPs and conjugated network materials for applications such as in selective gas adsorption, photocatalysis, energy storage, and sensing.

\subsection{Suggestions for Future Work}

On the basis of findings in this dissertation, the following are suggested for the future work. In Chapter 2 and 3, we have presented polymers based on novel building blocks like 2,1,3-dithienobenzochalcogenodiazole and $\gamma$-lactone-Pechmann dye. Fusing 2,1,3-benzochalcogeno- diazole with thiophene is a good design strategy as it will improve the planarity by restricting inter-annular rotation which allows a better $\pi$-electron delocalization, enhance the intermolecular interactions, improve the properties of light absorption and charge transportation but it decreases the electron withdrawing strength and consequently increases the LUMO energy level. Therefore, it can be increased by introducing an electron withdrawing groups via fluorination or replacing the carbon atoms at 4, 9 positions with nitrogen (to give an 
imine group). Thus we suggest this design strategies could give a novel acceptor building blocks, 4,9-difluorodithieno[3',2':3,4;2",3":5,6]benzo[1,2-c][1,2,5] chalcogenodiazole and dithiazolo[4',5':3,4;5",4":5,6]benzo[1,2-c][1,2,5] chalcogenodiazole as shown in Scheme 12. In addition to this, many more $\gamma$-lactonePechmann dye based polymers could be synthesized via changing the donor moiety which doesn't bear an alkyl group like 2,2'-bithiophene and thieno[3,2-b]thiophene and also changing the alkyl group, therefore, which may improve the $\pi-\pi$ stacking of the resulting polymer and the resulting polymer stability and charge carrier performance.

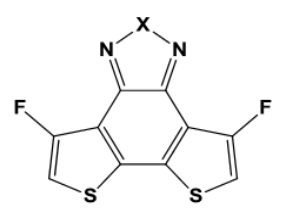

$\mathrm{X}=\mathrm{S}, \mathrm{O}$

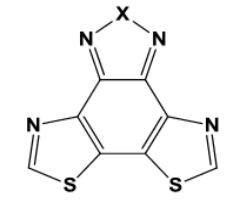

dithiazolo[4',5':3,4;5",4":5,6]benzo 4,9-difluorodithieno[3', $\left.2^{\prime}: 3,4 ; 2^{\prime \prime}, 3^{\prime \prime}: 5,6\right]$
benzo[1,2-c][1,2,5]chalcogenodiazole $[1,2-c][1,2,5]$ chalcogenodiazole



Scheme 13: Examples of proposed monomers and polymers for organic electronics applications. 
In Chapter 5, 6, and 7 of this dissertation presents the capability of direct C-H arylation coupling in synthesizing a good quality conjugated polymers and conjugated polymer networks like conjugated microporous polymers (CMPs). Therefore, based on these finding it could be suggested that direct $\mathrm{C}-\mathrm{H}$ arylation coupling has a great potential in replacing the already existing traditional coupling techniques for synthesizing semiconducting conjugated polymers and conjugated polymer networks. For instance, as shown in Scheme 13, direct C-H arylation coupling could be used in the synthesis of D-A conjugated polymers based on interesting and well-established building blocks like naphtho[1,2-c:5,6c']bis([1,2,5]thiadiazole). In addition to this, 2,5-dibromotetrathieno[3,2-a:2',3'c:3",2"-h:2"',3"'-j]phenazine based conjugated microporous polymers could be synthesized, as this building block avoids the structural twist between monomers as we have seen it in Chapter 7, when C-C coupling occurs between benzene and thiophene there is a twist which might affect the formation of an extended 2D-planar conjugated networks. This could be prevented by fusing the benzene in 8,11 dibromodithieno [3,2-a:2',3'-c]phenazine with thiophene (see Scheme 13). 

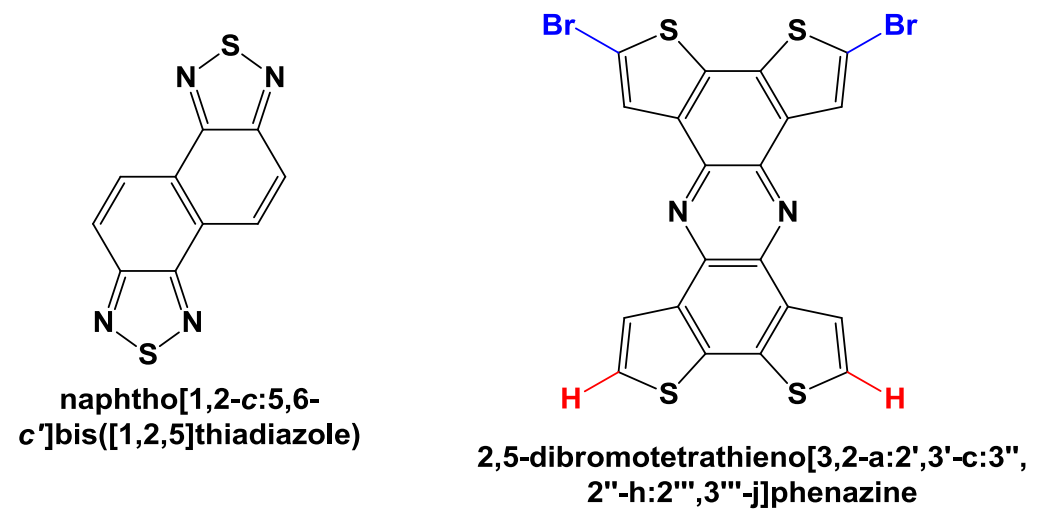

Scheme 14: Examples of proposed monomers for the synthesis of D-A conjugated polymers and conjugated microporous polymers (CMPs) via DAP. 


\section{REFERENCES}

(1) Schwoerer, M.; Wolf, H. C. Organic molecular solids; John Wiley \& Sons, 2007.

(2) Brédas, J.-L.; Beljonne, D.; Coropceanu, V.; Cornil, J. Chem. Rev. 2004, 104, 4971.

(3) Alan J. Heeger Angew. Chem. Int. Ed. 2001, 40, 2591

(4) Bradley, D. D. C. Polym. Int. 1991, 26, 3.

(5) Bradley, D. D. C.; Brown, A. R. Royal Society of Chemistry 1993, 120.

(6) Friend, R. H.; Burroughes, J. H.; Shimoda, T. Physics world 1999, 35.

(7) May, P. Physics world 1995, 52.

(8) Becquerel, A. E. Comptes Rendus 1839, 9, 561.

(9) http://en.wikipedia.org/wiki/Charles_Fritts.

(10) Chapin, D. M.; Fuller, C.; Pearson, G. J. Appl. Phys. 1954, 25, 676.

(11)https://commons.wikimedia.org/wiki/File:Best_Research-Cell_Efficiencies. png.

(12) Breeze, A.; Salomon, A.; Ginley, D.; Gregg, B.; Tillmann, H.; Hörhold, H.-H. Appl. Phys. Lett. 2002, 81, 3085.

(13) Halls, J.; Walsh, C.; Greenham, N.; Marseglia, E.; Friend, R.; Moratti, S.; Holmes, A. 1995.

(14) Jenekhe, S. A.; Yi, S. Appl. Phys. Lett. 2000, 77, 2635. 
(15) Sariciftci, N.; Braun, D.; Zhang, C.; Srdanov, V.; Heeger, A.; Stucky, G.; Wudl, F. Appl. Phys. Lett. 1993, 62, 585.

(16) Tang, C. W. Appl. Phys. Lett. 1986, 48, 183.

(17) Tsuzuki, T.; Shirota, Y.; Rostalski, J.; Meissner, D. Sol. Energy Mater. Sol. Cells 2000, 61, 1 .

(18) Uchida, S.; Xue, J.; Rand, B. P.; Forrest, S. R. Appl. Phys. Lett. 2004, 84, 4218.

(19) Wöhrle, D.; Meissner, D. Adv. Mater. 1991, 3, 129.

(20) Zhou, X.; Blochwitz, J.; Pfeiffer, M.; Nollau, A.; Fritz, T.; Leo, K. Adv. Funct. Mater. 2001, 11, 310.

(21) Winder, C.; Sariciftci, N. S. J. Mater. Chem. 2004, 14, 1077.

(22) Harrison, M.; Grüner, J.; Spencer, G. Physical Review B 1997, 55, 7831.

(23) Petterson, L.; Roman, L.; Inganäs, O. J. Appl. Phys. 1999, 86, 487.

(24) Rostalski, J.; Meissner, D. Sol. Energy Mater. Sol. Cells 2000, 63, 37.

(25) Hoppe, H.; Sariciftci, N. S. J. Mater. Res. 2004, 19, 1924.

(26) Hoppe, H.; Niggemann, M.; Winder, C.; Kraut, J.; Hiesgen, R.; Hinsch, A.; Meissner, D.; Sariciftci, N. S. Adv. Funct. Mater. 2004, 14, 1005.

(27) Peumans, P.; Uchida, S.; Forrest, S. R. Nature 2003, 425, 158.

(28) Yang, C.; Heeger, A. Synth. Met. 1996, 83, 85.

(29) Blom, P. W.; Mihailetchi, V. D.; Koster, L. J. A.; Markov, D. E. Adv. Mater. 2007, 19, 1551.

(30) Padinger, F.; Rittberger, R. S.; Sariciftci, N. S. Adv. Funct. Mater. 2003, 13, 85. 
(31) Kim, Y.-H.; Lee, S.-H.; Noh, J.; Han, S.-H. Thin Solid Films 2006, 510, 305.

(32) De Jong, M.; Van Ijzendoorn, L.; De Voigt, M. Appl. Phys. Lett. 2000, 77, 2255.

(33) Yim, K. H.; Zheng, Z.; Friend, R. H.; Huck, W. T.; Kim, J. S. Adv. Funct. Mater. 2008, 18, 2897.

(34) Pavlopoulou, E.; Fleury, G.; Deribew, D.; Cousin, F.; Geoghegan, M.; Hadziioannou, G. Org. Electron. 2013, 14, 1249.

(35) Chen, L. M.; Hong, Z.; Li, G.; Yang, Y. Adv. Mater. 2009, 21, 1434.

(36) Long, Y. Appl. Phys. Lett. 2009, 95, 193301.

(37) Ng, T.; Lo, M.; Liu, Z.; Wong, F.; Lai, S.; Fung, M.; Lee, C.; Lee, S. J. Appl. Phys. 2009, 106, 4501.

(38) Xu, Z.; Chen, L. M.; Yang, G.; Huang, C. H.; Hou, J.; Wu, Y.; Li, G.; Hsu, C. S.; Yang, Y. Adv. Funct. Mater. 2009, 19, 1227.

(39) Zhao, D.; Liu, P.; Sun, X.; Tan, S.; Ke, L.; Kyaw, A. Appl. Phys. Lett. 2009, 95, 153304.

(40) Menon, M.; Maheshkumar, M.; Sreekumar, K.; Kartha, C. S.; Vijayakumar, K. physica status solidi (a) 2012, 209, 199.

(41) Menon, M. R.; Maheshkumar, M.; Sreekumar, K.; Kartha, C. S.; Vijayakumar, K. Sol. Energy Mater. Sol. Cells 2010, 94, 2212.

(42) Krebs, F. C.; Gevorgyan, S. A.; Alstrup, J. J. Mater. Chem. 2009, 19, 5442.

(43) Scharber, M. C.; Mühlbacher, D.; Koppe, M.; Denk, P.; Waldauf, C.; Heeger, A. J.; Brabec, C. J. Advanced Materials 2006, 18, 789. 
(44) Kirchartz, T.; Taretto, K.; Rau, U. The Journal of Physical Chemistry C 2009, $113,17958$.

(45) Lilienfeld, J. E. 1930; Vol. No 1545175.

(46) Tsumura, A.; Koezuka, H.; Ando, T. Appl. Phys. Lett. 1986, 49, 1210.

(47) Koezuka, H.; Tsumura, A.; Ando, T. Synth. Met. 1987, 18, 699.

(48) Tseng, H. R.; Phan, H.; Luo, C.; Wang, M.; Perez, L. A.; Patel, S. N.; Ying, L.;

Kramer, E. J.; Nguyen, T. Q.; Bazan, G. C. Adv. Mater. 2014, 26, 2993.

(49) Kang, I.; Yun, H.-J.; Chung, D. S.; Kwon, S.-K.; Kim, Y.-H. Journal of the American Chemical Society 2013, 135, 14896.

(50) Sirringhaus, H.; Tessler, N.; Friend, R. H. Science 1998, 280, 1741.

(51) Dimitrakopoulos, C. D.; Malenfant, P. R. Adv. Mater. 2002, 14, 99.

(52) Halik, M.; Klauk, H.; Zschieschang, U.; Schmid, G.; Ponomarenko, S.; Kirchmeyer, S.; Weber, W. Adv. Mater. 2003, 15, 917.

(53) Lee, W. H.; Park, Y. D. Polymers 2014, 6, 1057.

(54) Horowitz, G.; Hajlaoui, R.; Bourguiga, R.; Hajlaoui, M. Synth. Met. 1999, 101, 401.

(55) Katz, H.; Bao, Z. The Journal of Physical Chemistry B 2000, 104, 671.

(56) Kline, R. J.; McGehee, M. D.; Kadnikova, E. N.; Liu, J.; Fréchet, J. M.; Toney, M. F. Macromolecules 2005, 38, 3312. 
(57) Zen, A.; Saphiannikova, M.; Neher, D.; Grenzer, J.; Grigorian, S.; Pietsch, U.; Asawapirom, U.; Janietz, S.; Scherf, U.; Lieberwirth, I. Macromolecules 2006, 39, 2162.

(58) Zhang, R.; Li, B.; Iovu, M. C.; Jeffries-EL, M.; Sauvé, G.; Cooper, J.; Jia, S.; Tristram-Nagle, S.; Smilgies, D. M.; Lambeth, D. N. J. Am. Chem. Soc. 2006, 128, 3480.

(59) Chen, Z.; Zheng, Y.; Yan, H.; Facchetti, A. J. Am. Chem. Soc. 2008, 131, 8.

(60) Bijleveld, J. C.; Zoombelt, A. P.; Mathijssen, S. G.; Wienk, M. M.; Turbiez, M.; de Leeuw, D. M.; Janssen, R. A. J. Am. Chem. Soc. 2009, 131, 16616.

(61) Mercier, L. G.; Leclerc, M. Acc. Chem. Res. 2013, 46, 1597.

(62) Azarian, D.; Dua, S. S.; Eaborn, C.; Walton, D. R. J. Organomet. Chem. 1976, 117, C55.

(63) Kosugi, M.; Sasazawa, K.; Shimizu, Y.; Migita, T. Chem. Lett. 1977, 6, 301.

(64) Kosugi, M.; Shimizu, Y.; Migita, T. Chem. Lett. 1977, 1423.

(65) Milstein, D.; Stille, J. J. Am. Chem. Soc. 1978, 100, 3636.

(66) Farina, V.; Baker, S. R.; Benigni, D. A.; Sapino, C. Tetrahedron Lett. 1988, 29, 5739.

(67) Espinet, P.; Echavarren, A. M. Angew. Chem. Int. Ed. 2004, 43, 4704.

(68) Farina, V.; Krishnan, B. J. Am. Chem. Soc. 1991, 113, 9585.

(69) Bao, Z.; Chan, W. K.; Yu, L. J. Am. Chem. Soc. 1995, 117, 12426.

(70) Miyaura, N.; Suzuki, A. J. Chem. Soc., Chem. Commun. 1979, 866. 
(71) Miyaura, N.; Yanagi, T.; Suzuki, A. Synth. Commun. 1981, 11, 513.

(72) Miyaura, N.; Yamada, K.; Suzuki, A. Tetrahedron Lett. 1979, 20, 3437.

(73) Kotha, S.; Lahiri, K.; Kashinath, D. Tetrahedron 2002, 58, 9633.

(74) Lloyd-Williams, P.; Giralt, E. Chem. Soc. Rev. 2001, 30, 145.

(75) Littke, A. F.; Fu, G. C. Angew. Chem. Int. Ed. 2002, 41, 4176.

(76) Rehahn, M.; Schlüter, A.-D.; Wegner, G.; Feast, W. J. Polymer 1989, 30, 1060.

(77) Braga, A. A.; Morgon, N. H.; Ujaque, G.; Maseras, F. J. Am. Chem. Soc. 2005, $127,9298$.

(78) Gorelsky, S. I.; Lapointe, D.; Fagnou, K. J. Am. Chem. Soc. 2008, 130, 10848.

(79) Lafrance, M.; Fagnou, K. J. Am. Chem. Soc. 2006, 128, 16496.

(80) Stuart, D. R.; Fagnou, K. Science 2007, 316, 1172.

(81) Campeau, L.-C.; Schipper, D. J.; Fagnou, K. J. Am. Chem. Soc. 2008, 130, 3266.

(82) Gorelsky, S. I.; Lapointe, D.; Fagnou, K. The Journal of organic chemistry 2011, 77, 658 .

(83) Wang, X.; Wang, M. Polymer Chemistry 2014, 5, 5784.

(84) Wang, X.; Wang, K.; Wang, M. Polymer Chemistry 2015, 6, 1846.

(85) Liang, Y.; Xu, Z.; Xia, J.; Tsai, S. T.; Wu, Y.; Li, G.; Ray, C.; Yu, L. Adv. Mater. 2010, 22 .

(86) Park, S. H.; Roy, A.; Beaupre, S.; Cho, S.; Coates, N.; Moon, J. S.; Moses, D.; Leclerc, M.; Lee, K.; Heeger, A. J. Nature photonics 2009, 3, 297. 
(87) Jo, J.; Pron, A.; Berrouard, P.; Leong, W. L.; Yuen, J. D.; Moon, J. S.; Leclerc, M.; Heeger, A. J. Advanced Energy Materials 2012, 2, 1397.

(88) Shaheen, S. E.; Brabec, C. J.; Sariciftci, N. S.; Padinger, F.; Fromherz, T.; Hummelen J. C. Appl. Phys. Lett. 2001, 78, 841.

(89) Brabec, C. J.; Shaheen, S. E.; Winder, C.; Sariciftci, N. S.; Denk, P. Appl. Phys. Lett. 2002, 80, 1288.

(90) Dennler, G.; Scharber, M. C.; Brabec, C. J. Adv. Mater. 2009, 21, 1323.

(91) Reyes-Reyes, M.; Kim, K.; Carroll, D. L. Appl. Phys. Lett. 2005, 87, 3.

(92) Kim, J. Y.; Kim, S. H.; Lee, H. H.; Lee, K.; Ma, W. L.; Gong, X.; Heeger, A. J. Adv. Mater. 2006, 18, 572.

(93) Ma, W. L.; Yang, C. Y.; Gong, X.; Lee, K.; Heeger, A. J. Adv. Funct. Mater. 2005, $15,1617$.

(94) Li, G.; Shrotriya, V.; Huang, J. S.; Yao, Y.; Moriarty, T.; Emery, K.; Yang, Y. Nature Mater. 2005, 4, 864.

(95) Assadi, A.; Svensson, C.; Willander, M.; Inganäs, O. Appl. Phys. Lett. 1988, 53, 195.

(96) Um, M.-C.; Jang, J.; Kang, J.; Hong, J.-P.; Lee, S. H.; Kim, J.-J.; Hong, J.-I. J. Mater. Chem. 2008, 18, 2234.

(97) Bao, Z.; Lovinger, A. J. Chem. Mater. 1999, 11, 2607.

(98) Chang, J.-F.; Sun, B.; Breiby, D. W.; Nielsen, M. M.; Sölling, T. I.; Giles, M.;

McCulloch, I.; Sirringhaus, H. Chem. Mater. 2004, 16, 4772. 
(99) Ong, B. S.; Wu, Y.; Liu, P.; Gardner, S. J. Am. Chem. Soc. 2004, 126, 3378.

(100) Ning, Z.; Fu, Y.; Tian, H. Energy \& Environmental Science 2010, 3, 1170.

(101) Lou, S. J.; Szarko, J. M.; Xu, T.; Yu, L.; Marks, T. J.; Chen, L. X. J. Am. Chem. Soc. 2011, 133, 20661.

(102) Bronstein, H.; Chen, Z.; Ashraf, R. S.; Zhang, W.; Du, J.; Durrant, J. R.; Shakya Tuladhar, P.; Song, K.; Watkins, S. E.; Geerts, Y. Journal of the American Chemical Society 2011, 133, 3272.

(103) Zhong, H.; Li, Z.; Buchaca-Domingo, E.; Rossbauer, S.; Watkins, S. E.; Stingelin, N.; Anthopoulos, T. D.; Heeney, M. Journal of Materials Chemistry A 2013, $1,14973$.

(104) Zhao, Y.; Guo, Y.; Liu, Y. Advanced Materials 2013, 25, 5372.

(105) Yuan, Y.; Giri, G.; Ayzner, A. L.; Zoombelt, A. P.; Mannsfeld, S. C.; Chen, J.; Nordlund, D.; Toney, M. F.; Huang, J.; Bao, Z. Nature communications 2014, 5.

(106) Li, Z. a.; Liu, Y.; Yu, G.; Wen, Y.; Guo, Y.; Ji, L.; Qin, J.; Li, Z. Advanced Functional Materials 2009, 19, 2677.

(107) Kim, R.; Amegadze, P. S.; Kang, I.; Yun, H. J.; Noh, Y. Y.; Kwon, S. K.; Kim, Y. H. Advanced Functional Materials 2013, 23, 5719.

(108) Li, J.; Zhao, Y.; Tan, H. S.; Guo, Y.; Di, C.-A.; Yu, G.; Liu, Y.; Lin, M.; Lim, S. H.; Zhou, Y. Scientific reports 2012, 2, 754.

(109) Gadisa, A. M., W.; Andersson, L. M.; Admassie, S.; Zhang,F.; Andersson, M. R.; Inganäs, O. Adv. Funct. Mater. 2007, 17, 3836. 
(110) Zhang, F.; Jespersen, K. G.; Björström, C.; Svensson, M.; Andersson, M. R.;

Sundström, V.; Magnusson, K.; Moons, E.; Yartsev, A.; Inganäs, O. Adv. Funct. Mater. 2006, 16, 667.

(111) Zheng, Q. J., B. J.; Sun, J.; Katz, H. E. J. Am. Chem. Soc. 2010, 132, 5394.

(112) Lewis, N. S. Science (Washington, DC, U. S.) 2007, 315, 798.

(113) Coffin, R. C. P., J.; Rogers, J.; Bazan, G. C. Nature Chem. 2009, 1, 657.

(114) Zoombelt, A. P. M., S. G. J.; Turbiez, M. G. R.; Wienk, M. M.; Janssen, R. A.

J. J. Mater. Chem. 2010, 20, 2240.

(115) Price, S. C. S., A. C.; Yang, L.; Zhou, H.; You, W. J. Am. Chem. Soc. 2011, $133,4625$.

(116) Boudreault, P. L. T. N., A.;; Leclerc, M. Chem. Mater. 2011, 23, 456.

(117) Chen, J. W. C., Y. Acc. Chem. Res. 2009, 42, 1709.

(118) Inganas, O. Z., F. L.; Andersson, M. R. Acc. Chem. Res. 2009, 42, 1731.

(119) Peet, J.; Heeger, A. J.; Bazan, G. C. Acc. Chem. Res. 2009, 42, 1700.

(120) Blouin, N. M., A.; Gendron, D.; Wakim, S.; Blair, E.; Neagu-Plesu, R.; Belletête, M.; Durocher, G.; Tao, Y.; Leclerc,M.; Michaud, A.; Leclerc, M. A. Adv. Mater. 2007, 19, 2295.

(121) Peet, J.; Kim, J.; Coates, N.; Ma, W.; Moses, D.; Heeger, A. J.; Bazan, G. Nat. Mater. 2007, 6, 497.

(122) Svensson, M.; Zhang, F.; Veenstra, S.; Verhees, W.; Hummelen, J.; Kroon, J.; Inganas, O.; Andersson, M. R. Adv. Mater. 2003, 15, 988. 
(123) Wang, E.; Wang, L.; Lan, L.; Luo, C.; Zhuang, W.; Peng, J.; Cao, Y. Appl. Phys. Lett. 2008, 92, 033307

(124) Nie, W.; MacNeill, C. M.; Li, Y.; Noftle, R. E.; Carroll, D. L.; Coffin, R. C. Macromol. Rapid Commun. 2011, 32, 1163.

(125) Zhang, L. H., C.; Chen, J.; Yuan, P.; Huang, L.; Zhang, C.; Cai, W.; Liu, Z.; Cao, Y. Macromolecules 2010, 43, 9771.

(126) Zhao, W. C., W.; Xu, R.; Yang, W.; Gong, X.; Wu, H.; Cao, Y. Polymer 2010, $51,3196$.

(127) Blouin, N. M., A.; Gendron, D.; Wakim, S.; Blair, E.; Neagu-Plesu, R.; Belletête, M.; Durocher, G.; Tao, Y.; Leclerc, M. J.Am.Chem.Soc. 2007, 130, 732.

(128) Gendron, D. M., P.O.; Najari, A.; Leclerc, M. Macromol. Rapid Commun. 2010, 31, 1090 .

(129) Liu, Y.; Zhao, J.; Li, Z.; Mu, C.; Ma, W.; Hu, H.; Jiang, K.; Lin, H.; Ade, H.; Yan, H. Nature communications 2014, 5.

(130) Zhao, W.; Qian, D.; Zhang, S.; Li, S.; Inganäs, O.; Gao, F.; Hou, J. Adv. Mater. 2016, 28, 4734.

(131) Kanimozhi, C.; Yaacobi-Gross, N.; Chou, K. W.; Amassian, A.; Anthopoulos, T. D.; Patil, S. Journal of the American Chemical Society 2012, 134, 16532.

(132) Lee, J.; Han, A.-R.; Yu, H.; Shin, T. J.; Yang, C.; Oh, J. H. Journal of the American Chemical Society 2013, 135, 9540. 
(133) Lei, T.; Cao, Y.; Fan, Y.; Liu, C.-J.; Yuan, S.-C.; Pei, J. J. Am. Chem. Soc. 2011, 133, 6099.

(134) Lei, T.; Dou, J. H.; Pei, J. Advanced Materials 2012, 24, 6457.

(135) Mei, J.; Kim, D. H.; Ayzner, A. L.; Toney, M. F.; Bao, Z. J. Am. Chem. Soc. 2011, 133, 20130.

(136) Van Pruissen, G. W.; Gholamrezaie, F.; Wienk, M. M.; Janssen, R. A. Journal of Materials Chemistry 2012, 22, 20387.

(137) Koizumi, Y.; Ide, M.; Saeki, A.; Vijayakumar, C.; Balan, B.; Kawamoto, M.; Seki, S. Polymer Chemistry 2013, 4, 484.

(138) Lei, T.; Dou, J.-H.; Cao, X.-Y.; Wang, J.-Y.; Pei, J. Journal of the American Chemical Society 2013, 135, 12168.

(139) Cai, Z.; Luo, H.; Qi, P.; Wang, J.; Zhang, G.; Liu, Z.; Zhang, D. Macromolecules 2014, 47, 2899.

(140) Kowalski, S.; Allard, S.; Scherf, U. ACS Macro Letters 2012, 1, 465.

(141) Kowalski, S.; Allard, S.; Scherf, U. Macromolecular rapid communications 2015, 36, 1061.

(142) Chang, S. W.; Waters, H.; Kettle, J.; Kuo, Z. R.; Li, C. H.; Yu, C. Y.; Horie, M. Macromolecular rapid communications 2012, 33, 1927.

(143) Homyak, P.; Liu, Y.; Liu, F.; Russel, T. P.; Coughlin, E. B. Macromolecules 2015, 48, 6978 . 
(144) Shao, J.; Wang, G.; Wang, K.; Yang, C.; Wang, M. Polymer Chemistry 2015, $6,6836$.

(145) Sun, M.; Wang, W.; Lv, W.; Lu, M.; Yan, S.; Liang, L.; Ling, Q. Synthetic Metals 2015, 209, 412.

(146) Pouliot, J.-R.; Sun, B.; Leduc, M.; Najari, A.; Li, Y.; Leclerc, M. Polymer Chemistry 2015, 6, 278.

(147) Grenier, F.; Berrouard, P.; Pouliot, J.-R.; Tseng, H.-R.; Heeger, A. J.; Leclerc, M. Polymer Chemistry 2013, 4, 1836.

(148) Elsawy, W.; Son, M.; Jang, J.; Kim, M. J.; Ji, Y.; Kim, T.-W.; Ko, H. C.; Elbarbary, A.; Ham, M.-H.; Lee, J.-S. ACS Macro Letters 2015, 4, 322.

(149) Grenier, F.; Aïch, B. R.; Lai, Y.-Y.; Guérette, M.; Holmes, A. B.; Tao, Y.; Wong, W. W.; Leclerc, M. Chemistry of Materials 2015, 27, 2137.

(150) Huang, J.; Wang, K.; Gupta, S.; Wang, G.; Yang, C.; Mushrif, S. H.; Wang, M. Journal of Polymer Science Part A: Polymer Chemistry 2016.

(151) Wang, K.; Wang, G.; Wang, M. Macromolecular rapid communications 2015 , $36,2162$.

(152) Bohra, H.; Shao, J.; Huang, S.; Wang, M. Tetrahedron Lett. 2016.

(153) J. Huang; K. Wang; S. Gupta; G. Wang; C. Yang; S. H. Mushrif; M. Wang J. Polym. Sci. Part A: Polym. Chem. 2016.

(154) Holliday, S.; Donaghey, J. E.; McCulloch, I. Chem. Mater. 2013, 26, 647.

(155) Guo, X.; Baumgarten, M.; Müllen, K. Prog. Polym. Sci. 2013, 38, 1832. 
(156) Usta, H.; Facchetti, A.; Marks, T. J. Acc. Chem. Res. 2011, 44, 501.

(157) Anthony, J. E.; Facchetti, A.; Heeney, M.; Marder, S. R.; Zhan, X. Adv. Mater. 2010, 22, 3876.

(158) Jung, B. J.; Tremblay, N. J.; Yeh, M.-L.; Katz, H. E. Chem. Mater. 2010, 23, 568.

(159) Shin, R. Y.; Sonar, P.; Siew, P. S.; Chen, Z.-K.; Sellinger, A. The Journal of organic chemistry 2009, 74, 3293.

(160) Li, J.; Zhao, Y.; Tan, H. S.; Guo, Y.; Di, C.-A.; Yu, G.; Liu, Y.; Lin, M.; Lim, S. H.; Zhou, Y.; Haibin, S.; Ong, B. S. Scientific reports 2012, 2.

(161) Boudreault, P.-L. T.; Najari, A.; Leclerc, M. Chem. Mater. 2010, 23, 456.

(162) Chen, J.; Cao, Y. Acc. Chem. Res. 2009, 42, 1709.

(163) Inganäs, O.; Zhang, F.; Andersson, M. R. Acc. Chem. Res. 2009, 42, 1731.

(164) Peet, J.; Heeger, A. J.; Bazan, G. C. Acc. Chem. Res. 2009, 42, 1700.

(165) Svensson, M.; Zhang, F.; Veenstra, S. C.; Verhees, W. J.; Hummelen, J. C.; Kroon, J. M.; Inganäs, O.; Andersson, M. R. Adv. Mater. 2003, 15, 988.

(166) Blouin, N.; Michaud, A.; Leclerc, M. Adv. Mater. 2007, 19, 2295.

(167) Peet, J.; Kim, J.; Coates, N. E.; Ma, W. L.; Moses, D.; Heeger, A. J.; Bazan, G. C. Nature materials 2007, 6, 497.

(168) Wang, E.; Wang, L.; Lan, L.; Luo, C.; Zhuang, W.; Peng, J.; Cao, Y. Appl. Phys. Lett. 2008, 92, 033307. 
(169) Nie, W.; MacNeill, C. M.; Li, Y.; Noftle, R. E.; Carroll, D. L.; Coffin, R. Macromol. Rapid Commun. 2011, 32, 1163.

(170) Zhang, L.; He, C.; Chen, J.; Yuan, P.; Huang, L.; Zhang, C.; Cai, W.; Liu, Z.; Cao, Y. Macromolecules 2010, 43, 9771.

(171) Zhao, W.; Cai, W.; Xu, R.; Yang, W.; Gong, X.; Wu, H.; Cao, Y. Polymer $\mathbf{2 0 1 0}, 51,3196$.

(172) Blouin, N.; Michaud, A.; Gendron, D.; Wakim, S.; Blair, E.; Neagu-Plesu, R.; Belletete, M.; Durocher, G.; Tao, Y.; Leclerc, M. J. Am. Chem. Soc. 2008, 130, 732. (173) Gendron, D.; Morin, P. O.; Najari, A.; Leclerc, M. Macromol. Rapid Commun. 2010, 31, 1090 .

(174) Xiao, K.; Liu, Y.; Qi, T.; Zhang, W.; Wang, F.; Gao, J.; Qiu, W.; Ma, Y.; Cui, G.; Chen, S. J. Am. Chem. Soc. 2005, 127, 13281.

(175) Zhang, S.; Guo, Y.; Zhang, Y.; Liu, R.; Li, Q.; Zhan, X.; Liu, Y.; Hu, W. Chem. Commun. 2010, 46, 2841.

(176) Wu, W.; Liu, Y.; Zhu, D. Chem. Soc. Rev. 2010, 39, 1489.

(177) Liu, Y.; Liu, Y.; Zhan, X. Macromol. Chem. Phys. 2011, 212, 428.

(178) Wang, H.; Shi, Q.; Lin, Y.; Fan, H.; Cheng, P.; Zhan, X.; Li, Y.; Zhu, D. Macromolecules 2011, 44, 4213.

(179) Mei, C.-Y.; Liang, L.; Zhao, F.-G.; Wang, J.-T.; Yu, L.-F.; Li, Y.-X.; Li, W.S. Macromolecules 2013, 46, 7920. 
(180) Hu, S.; Bao, X.; Liu, Z.; Wang, T.; Du, Z.; Wen, S.; Wang, N.; Han, L.; Yang, R. Org. Electron. 2014, 15, 3601.

(181) Huang, J.; Zhu, Y.; Chen, J.; Zhang, L.; Peng, J.; Cao, Y. Macromol. Rapid Commun. 2014, 35, 1960.

(182) Park, H. J.; Lee, Y.; Jo, J. W.; Jo, W. H. Polymer Chemistry 2012, 3, 2928.

(183) Cheng, Y.-J.; Ho, Y.-J.; Chen, C.-H.; Kao, W.-S.; Wu, C.-E.; Hsu, S.-L.; Hsu, C.-S. Macromolecules 2012, 45, 2690.

(184) Planells, M.; Nikolka, M.; Hurhangee, M.; Tuladhar, P. S.; White, A. J.; Durrant, J. R.; Sirringhaus, H.; McCulloch, I. Journal of Materials Chemistry C 2014, 2, 8789 .

(185) Efrem, A.; Lim, C.-J.; Lu, Y.; Ng, S.-C. Tetrahedron Lett. 2014, 55, 4849.

(186) Jiang, J.-M.; Yang, P.-A.; Hsieh, T.-H.; Wei, K.-H. Macromolecules 2011, 44, 9155.

(187) Liu, B.; Chen, X.; Zou, Y.; Xiao, L.; Xu, X.; He, Y.; Li, L.; Li, Y. Macromolecules 2012, 45, 6898.

(188) Jiang, J. M.; Yang, P. A.; Yu, C. M.; Lin, H. K.; Huang, K. C.; Wei, K. H. J. Polym. Sci., Part A: Polym. Chem. 2012, 50, 3960.

(189) Bijleveld, J. C.; Shahid, M.; Gilot, J.; Wienk, M. M.; Janssen, R. A. Adv. Funct. Mater. 2009, 19, 3262.

(190) Padhy, H.; Huang, J. H.; Sahu, D.; Patra, D.; Kekuda, D.; Chu, C. W.; Lin, H. C. J. Polym. Sci., Part A: Polym. Chem. 2010, 48, 4823. 
(191) Jiang, J.-M.; Yang, P.-A.; Chen, H.-C.; Wei, K.-H. Chem. Commun. 2011, 47, 8877.

(192) Lan, S.-C.; Yang, P.-A.; Zhu, M.-J.; Yu, C.-M.; Jiang, J.-M.; Wei, K.-H. Polymer Chemistry 2013, 4, 1132.

(193) Wang, X.; Jiang, P.; Chen, Y.; Luo, H.; Zhang, Z.; Wang, H.; Li, X.; Yu, G.; Li, Y. Macromolecules 2013, 46, 4805.

(194) Ni, J.-S.; You, J.-H.; Hung, W.-I.; Kao, W.-S.; Chou, H.-H.; Lin, J. T. ACS applied materials \& interfaces 2014, 6, 22612.

(195) Casalini, R.; Tsang, S. W.; Deininger, J. J.; Arroyave, F. A.; Reynolds, J. R.; So, F. The Journal of Physical Chemistry C 2013, 117, 13798.

(196) Choi, M.-H.; Song, K. W.; Moon, D. K. Polymer Chemistry 2015, 6, 2636.

(197) Wu, Q.; Ren, S.; Wang, M.; Qiao, X.; Li, H.; Gao, X.; Yang, X.; Zhu, D. Adv. Funct. Mater. 2013, 23, 2277.

(198) Arroyave, F. A.; Richard, C. A.; Reynolds, J. R. Org. Lett. 2012, 14, 6138.

(199) Mancilha, F. S.; DaSilveira Neto, B. A.; Lopes, A. S.; Moreira, P. F.; Quina, F. H.; Gonçalves, R. S.; Dupont, J. Eur. J. Org. Chem. 2006, 2006, 4924.

(200) Lou, X.; van Dongen, J. L.; Braeken, Y.; Brebels, J.; van Pruissen, G. W.; Li, W.; Wienk, M. M.; Janssen, R. A. Polymer Chemistry 2014, 5, 558.

(201) Shi, S.; Shi, K.; Chen, S.; Qu, R.; Wang, L.; Wang, M.; Yu, G.; Li, X.; Wang, H. J. Polym. Sci., Part A: Polym. Chem. 2014, 52, 2465. 
(202) Lei, T.; Cao, Y.; Zhou, X.; Peng, Y.; Bian, J.; Pei, J. Chem. Mater. 2012, 24, 1762.

(203) Qi, B.; Wang, J. PCCP 2013, 15, 8972.

(204) Osaka, I.; Zhang, R.; Sauvé, G.; Smilgies, D.-M.; Kowalewski, T.; McCullough, R. D. J. Am. Chem. Soc. 2009, 131, 2521.

(205) Garnier, F.; Horowitz, G.; Fichou, D. Synth. Met. 1989, 28, 705.

(206) Liang, Y.; Wu, Y.; Feng, D.; Tsai, S.-T.; Son, H.-J.; Li, G.; Yu, L. J. Am. Chem. Soc. 2008, 131, 56.

(207) Carsten, B.; Szarko, J. M.; Son, H. J.; Wang, W.; Lu, L.; He, F.; Rolczynski, B. S.; Lou, S. J.; Chen, L. X.; Yu, L. J. Am. Chem. Soc. 2011, 133, 20468.

(208) Huang, F.; Chen, K.-S.; Yip, H.-L.; Hau, S. K.; Acton, O.; Zhang, Y.; Luo, J.; Jen, A. K.-Y. J. Am. Chem. Soc. 2009, 131, 13886.

(209) Cheng, Y.-J.; Yang, S.-H.; Hsu, C.-S. Chem. Rev. 2009, 109, 5868.

(210) Yuen, J. D.; Fan, J.; Seifter, J.; Lim, B.; Hufschmid, R.; Heeger, A. J.; Wudl, F. J. Am. Chem. Soc. 2011, 133, 20799.

(211) Zhan, X.; Facchetti, A.; Barlow, S.; Marks, T. J.; Ratner, M. A.; Wasielewski, M. R.; Marder, S. R. Adv. Mater. 2011, 23, 268.

(212) Zhan, X.; Tan, Z. a.; Domercq, B.; An, Z.; Zhang, X.; Barlow, S.; Li, Y.; Zhu, D.; Kippelen, B.; Marder, S. R. J. Am. Chem. Soc. 2007, 129, 7246. 
(213) Tsao, H. N.; Cho, D. M.; Park, I.; Hansen, M. R.; Mavrinskiy, A.; Yoon, D. Y.; Graf, R.; Pisula, W.; Spiess, H. W.; Müllen, K. J. Am. Chem. Soc. 2011, 133, 2605.

(214) Ong, K. H.; Lim, S. L.; Tan, H. S.; Wong, H. K.; Li, J.; Ma, Z.; Moh, L. C.; Lim, S. H.; de Mello, J. C.; Chen, Z. K. Adv. Mater. 2011, 23, 1409.

(215) Yuen, J. D.; Kumar, R.; Zakhidov, D.; Seifter, J.; Lim, B.; Heeger, A. J.; Wudl, F. Adv. Mater. 2011, 23, 3780 .

(216) Li, J.; Zhao, Y.; Tan, H. S.; Guo, Y.; Di, C.-A.; Yu, G.; Liu, Y.; Lin, M.; Lim, S. H.; Zhou, Y. Scientific reports 2012, 2.

(217) Chen, Z.; Lee, M. J.; Shahid Ashraf, R.; Gu, Y.; Albert-Seifried, S.; Meedom Nielsen, M.; Schroeder, B.; Anthopoulos, T. D.; Heeney, M.; McCulloch, I. Adv. Mater. 2012, 24, 647.

(218) Zhang, X.; Richter, L. J.; DeLongchamp, D. M.; Kline, R. J.; Hammond, M. R.; McCulloch, I.; Heeney, M.; Ashraf, R. S.; Smith, J. N.; Anthopoulos, T. D. J. Am. Chem. Soc. 2011, 133, 15073.

(219) Cai, Z.; Guo, Y.; Yang, S.; Peng, Q.; Luo, H.; Liu, Z.; Zhang, G.; Liu, Y.; Zhang, D. Chem. Mater. 2013, 25, 471.

(220) Cai, Z.; Liu, Z.; Luo, H.; Qi, P.; Zhang, G.; Zhang, D. Chin. J. Chem . 2014, 32,788 .

(221) Pechmann, H. Ber. Dtsch. Chem. Ges 1882, 15, 881.

(222) Klingsberg, E. Chem. Rev. 1954, 54, 59. 
(223) Fang, C. S.; Bergmann, W. The Journal of Organic Chemistry 1951, 16, 1231.

(224) Begley, M. J.; Crombie, L.; Griffiths, G. L.; Jones, R. C.; Rahmani, M. J. Chem. Soc., Chem. Commun. 1981, 823.

(225) Silver, J.; Ahmet, M. T.; Bowden, K.; Miller, J. R.; Rahmat, S.; Reynolds, C. A.; Bashall, A.; McPartlin, M.; Trotter, J. J. Mater. Chem. 1994, 4, 1201.

(226) Norsten, T. B.; Kantchev, E. A. B.; Sullivan, M. B. Org. Lett. 2010, 12, 4816.

(227) Kantchev, E. A. B.; Norsten, T. B.; Tan, M. L.; Ng, J. J.; Sullivan, M. B. Chemistry-A European Journal 2012, 18, 695.

(228) Hayashi, M.; Toshimitsu, F.; Sakamoto, R.; Nishihara, H. J. Am. Chem. Soc. 2011, 133, 14518.

(229) Fukazawa, A.; Adachi, M.; Nakakura, K.; Saito, S.; Yamaguchi, S. Chem. Commun. 2013, 49, 7117.

(230) Wang, X.-Y.; Zhang, M.-W.; Zhuang, F.-D.; Wang, J.-Y.; Pei, J. Polymer Chemistry 2016, 7, 2264.

(231) Kantchev, E. A. B.; Norsten, T. B.; Tan, M. L.; Ng, J. J.; Sullivan, M. B. Chemistry-A European Journal 2012, 18, 695.

(232) Wang, X.-Y.; Zhuang, F.-D.; Wang, J.-Y.; Pei, J. Chem. Commun. 2015, 51, 17532.

(233) Tsai, J.-H.; Lee, W.-Y.; Chen, W.-C.; Yu, C.-Y.; Hwang, G.-W.; Ting, C. Chem. Mater. 2010, 22, 3290. 
(234) Zhang, S.; Ye, L.; Zhao, W.; Liu, D.; Yao, H.; Hou, J. Macromolecules 2014, $47,4653$.

(235) Liu, Y.; Liu, M.; Jen, A. Y. Acta Polym. 1999, 50, 105.

(236) Lu, C.; Wu, H. C.; Chiu, Y. C.; Lee, W. Y.; Chen, W. C. Macromolecules 2012, 45, 3047.

(237) Huo, L.; Zhang, S.; Guo, X.; Xu, F.; Li, Y.; Hou, J. Angew. Chem. 2011, 123, 9871.

(238) Warnan, J.; El Labban, A.; Cabanetos, C.; Hoke, E. T.; Shukla, P. K.; Risko, C.; Brédas, J.-L.; McGehee, M. D.; Beaujuge, P. M. Chem. Mater. 2014, 26, 2299. (239) Wang, C.; Dong, H.; Hu, W.; Liu, Y.; Zhu, D. Chem. Rev. 2011, 112, 2208.

(240) Deng, Y.; Chen, Y.; Zhang, X.; Tian, H.; Bao, C.; Yan, D.; Geng, Y.; Wang, F. Macromolecules 2012, 45, 8621.

(241) Mei, J.; Diao, Y.; Appleton, A. L.; Fang, L.; Bao, Z. J. Am. Chem. Soc. 2013, $135,6724$.

(242) Facchetti, A. Nature materials 2013, 12, 598.

(243) Tsao, H. N.; Müllen, K. Chem. Soc. Rev. 2010, 39, 2372.

(244) Takeda, Y.; Andrew, T. L.; Lobez, J. M.; Mork, A. J.; Swager, T. M. Angew. Chem. Int. Ed. 2012, 51, 9042.

(245) Koster, L.; Shaheen, S. E.; Hummelen, J. C. Advanced Energy Materials 2012, $2,1246$.

(246) Li, J.; Grimsdale, A. C. Chem. Soc. Rev. 2010, 39, 2399. 
(247) Chen, H.-Y.; Hou, J.; Zhang, S.; Liang, Y.; Yang, G.; Yang, Y.; Yu, L.; Wu, Y.; Li, G. Nature photonics 2009, 3, 649.

(248) He, Z.; Zhong, C.; Su, S.; Xu, M.; Wu, H.; Cao, Y. Nature Photonics 2012, 6, 591.

(249) You, J.; Dou, L.; Yoshimura, K.; Kato, T.; Ohya, K.; Moriarty, T.; Emery, K.; Chen, C.-C.; Gao, J.; Li, G. Nature communications 2013, 4, 1446.

(250) Grimsdale, A. C.; Leok Chan, K.; Martin, R. E.; Jokisz, P. G.; Holmes, A. B. Chem. Rev. 2009, 109, 897.

(251) Huang, F.; Wu, H.; Cao, Y. Chem. Soc. Rev. 2010, 39, 2500.

(252) Głowacki, E. D.; Voss, G.; Demirak, K.; Havlicek, M.; Sünger, N.; Okur, A. C.; Monkowius, U.; Gąsiorowski, J.; Leonat, L.; Sariciftci, N. S. Chem. Commun. 2013, 49, 6063.

(253) Zambounis, J.; Hao, Z.; Iqbal, A. Nature 1997, 388, 131.

(254) Jakob, S.; Moreno, A.; Zhang, X.; Bertschi, L.; Smith, P.; Schluter, A. D.; Sakamoto, J. Macromolecules 2010, 43, 7916.

(255) Bundgaard, E.; Hagemann, O.; Bjerring, M.; Nielsen, N. C.; Andreasen, J. W.; Andreasen, B.; Krebs, F. C. Macromolecules 2012, 45, 3644.

(256) Helgesen, M.; Gevorgyan, S. A.; Krebs, F. C.; Janssen, R. A. Chem. Mater. 2009, 21, 4669 .

(257) Helgesen, M.; Bjerring, M.; Nielsen, N. C.; Krebs, F. C. Chem. Mater. 2010, 22,5617 . 
(258) Liu, Y.; Scully, S. R.; McGehee, M. D.; Liu, J.; Luscombe, C. K.; Frechet, J. M.; Shaheen, S. E.; Ginley, D. S. The Journal of Physical Chemistry B 2006, 110, 3257.

(259) Liu, C.; Xu, W.; Xue, Q.; Cai, P.; Ying, L.; Huang, F.; Cao, Y. Dyes and Pigments 2016, 125, 54.

(260) Liu, C.; Dong, S.; Cai, P.; Liu, P.; Liu, S.; Chen, J.; Liu, F.; Ying, L.; Russell, T. P.; Huang, F. ACS applied materials \& interfaces 2015, 7, 9038.

(261) Chen, T. L.; Chen, J. J.-A.; Catane, L.; Ma, B. Org. Electron. 2011, 12, 1126.

(262) Sun, B.; Hong, W.; Aziz, H.; Li, Y. J. Mater. Chem. 2012, 22, 18950.

(263) Guo, C.; Quinn, J.; Sun, B.; Li, Y. Journal of Materials Chemistry C 2015, 3, 5226.

(264) Zhang, X.-X.; Sadighi, J. P.; Mackewitz, T. W.; Buchwald, S. L. J. Am. Chem. Soc. 2000, 122, 7606.

(265) Sadighi, J. P.; Singer, R. A.; Buchwald, S. L. J. Am. Chem. Soc. 1998, 120, 4960.

(266) Fukumoto, H.; Nakajima, H.; Kojima, T.; Yamamoto, T. Materials 2014, 7, 2030.

(267) Yamamoto, T.; Yoshizawa, M.; Mahmut, A.; Abe, M.; Kuroda, S. i.; Imase, T.; Sasaki, S. J. Polym. Sci., Part A: Polym. Chem. 2005, 43, 6223.

(268) Horie, M.; Yamaguchi, I.; Yamamoto, T. Macromolecules 2006, 39, 7493. 
(269) Srinivasan, N.; Yurek-George, A.; Ganesan, A. Molecular diversity 2005, 9, 291.

(270) Li, S.-G.; Jiang, K.-J.; Huang, J.-H.; Yang, L.-M.; Song, Y.-L. Chem. Commun. 2014, 50, 4309.

(271) Trost, B. M.; Osipov, M. Angew. Chem. Int. Ed. 2013, 52, 9176.

(272) Liu, Y.; Liu, M.; Jen, A.-Y. Acta Polym. 1999, 50, 105.

(273) Yang, L.; Yu, Y.; Gong, Y.; Li, J.; Ge, F.; Jiang, L.; Gao, F.; Dan, Y. Polymer Chemistry 2015, 6, 7005.

(274) Carsten, B.; He, F.; Son, H. J.; Xu, T.; Yu, L. Chem. Rev. 2011, 111, 1493.

(275) Okamoto, K.; Luscombe, C. K. Polymer Chemistry 2011, 2, 2424.

(276) Sakamoto, J.; Rehahn, M.; Wegner, G.; Schlüter, A. D. Macromol. Rapid Commun. 2009, 30, 653.

(277) Amatore, C.; Jutand, A.; Le Duc, G. Angew. Chem. 2012, 124, 1408.

(278) Ball, L. T.; Lloyd-Jones, G. C.; Russell, C. A. Science 2012, 337, 1644.

(279) Alberico, D.; Scott, M. E.; Lautens, M. Chem. Rev. 2007, 107, 174.

(280) Wencel-Delord, J.; Glorius, F. Nature chemistry 2013, 5, 369.

(281) Khlyabich, P. P.; Burkhart, B.; Rudenko, A. E.; Thompson, B. C. Polymer 2013, 54, 5267.

(282) Okamoto, K.; Zhang, J.; Housekeeper, J. B.; Marder, S. R.; Luscombe, C. K. Macromolecules 2013, 46, 8059.

(283) Wang, K.; Wang, M. Curr. Org. Chem. 2013, 17, 999. 
(284) Rudenko, A. E.; Thompson, B. C. J. Polym. Sci., Part A: Polym. Chem. 2015, $53,135$.

(285) Zhou, H.; Yang, L.; Stuart, A. C.; Price, S. C.; Liu, S.; You, W. Angew. Chem. 2011, 123, 3051.

(286) Jheng, J. F.; Lai, Y. Y.; Wu, J. S.; Chao, Y. H.; Wang, C. L.; Hsu, C. S. Adv. Mater. 2013, 25, 2445.

(287) You, J.; Chen, C. C.; Hong, Z.; Yoshimura, K.; Ohya, K.; Xu, R.; Ye, S.; Gao, J.; Li, G.; Yang, Y. Adv. Mater. 2013, 25, 3973.

(288) Chen, Y.-L.; Kao, W.-S.; Tsai, C.-E.; Lai, Y.-Y.; Cheng, Y.-J.; Hsu, C.-S. Chem. Commun. 2013, 49, 7702.

(289) Zhang, Y.; Chien, S.-C.; Chen, K.-S.; Yip, H.-L.; Sun, Y.; Davies, J. A.; Chen, F.-C.; Jen, A. K.-Y. Chem. Commun. 2011, 47, 11026.

(290) Stuart, A. C.; Tumbleston, J. R.; Zhou, H.; Li, W.; Liu, S.; Ade, H.; You, W. J. Am. Chem. Soc. 2013, 135, 1806.

(291) Price, S. C.; Stuart, A. C.; Yang, L.; Zhou, H.; You, W. J. Am. Chem. Soc. 2011, 133, 4625 .

(292) Bronstein, H.; Frost, J. M.; Hadipour, A.; Kim, Y.; Nielsen, C. B.; Ashraf, R. S.; Rand, B. P.; Watkins, S.; McCulloch, I. Chem. Mater. 2013, 25, 277.

(293) Zhang, J.; Chen, W.; Rojas, A. J.; Jucov, E. V.; Timofeeva, T. V.; Parker, T. C.; Barlow, S.; Marder, S. R. Journal of the American Chemical Society 2013, 135, 16376. 
(294) Zhang, X.; Gao, Y.; Li, S.; Shi, X.; Geng, Y.; Wang, F. Journal of Polymer Science Part A: Polymer Chemistry 2014, 52, 2367.

(295) Zhang, J.; Parker, T. C.; Chen, W.; Williams, L.; Khrustalev, V. N.; Jucov, E. V.; Barlow, S.; Timofeeva, T. V.; Marder, S. The Journal of organic chemistry 2015. (296) Chen, Z.; Cai, P.; Chen, J.; Liu, X.; Zhang, L.; Lan, L.; Peng, J.; Ma, Y.; Cao, Y. Adv. Mater. 2014, 26, 2586.

(297) Dou, L.; Chen, C.-C.; Yoshimura, K.; Ohya, K.; Chang, W.-H.; Gao, J.; Liu, Y.; Richard, E.; Yang, Y. Macromolecules 2013, 46, 3384.

(298) Kim, Y.; Cook, S.; Kirkpatrick, J.; Nelson, J.; Durrant, J.; Bradley, D.; Giles, M.; Heeney, M.; Hamilton, R.; McCulloch, I. The Journal of Physical Chemistry C 2007, 111, 8137.

(299) Kettle, J.; Horie, M.; Majewski, L.; Saunders, B.; Tuladhar, S.; Nelson, J.; Turner, M. Sol. Energy Mater. Sol. Cells 2011, 95, 2186.

(300) Ashimine, T.; Yasuda, T.; Saito, M.; Nakamura, H.; Tsutsui, T. Japanese Journal of Applied Physics 2008, 47, 1760.

(301) Qiao, Y.; Guo, Y.; Yu, C.; Zhang, F.; Xu, W.; Liu, Y.; Zhu, D. J. Am. Chem. Soc. 2012, 134, 4084.

(302) Efrem, A.; Lei, Y.; Wu, B.; Wang, M.; Ng, S. C.; Ong, B. S. Dyes and Pigments 2016, 129, 90 .

(303) Wang, Y.; Xin, X.; Lu, Y.; Xiao, T.; Xu, X.; Zhao, N.; Hu, X.; Ong, B. S.; Ng, S. C. Macromolecules 2013, 46, 9587. 
(304) Morin, P.-O.; Bura, T.; Sun, B.; Gorelsky, S. I.; Li, Y.; Leclerc, M. ACS Macro Letters 2014, 4, 21.

(305) Matsidik, R.; Komber, H.; Luzio, A.; Caironi, M.; Sommer, M. J. Am. Chem. Soc. 2015, 137, 6705 .

(306) Guérette, M.; Najari, A.; Maltais, J.; Pouliot, J. R.; Dufresne, S.; Simoneau, M.; Besner, S.; Charest, P.; Leclerc, M. Advanced Energy Materials 2016.

(307) Koldemir, U.; Puniredd, S. R.; Wagner, M.; Tongay, S.; McCarley, T. D.; Kamenov, G. D.; Müllen, K.; Pisula, W.; Reynolds, J. R. Macromolecules 2015, 48, 6369.

(308) Park, J. K.; Jo, J.; Seo, J. H.; Moon, J. S.; Park, Y. D.; Lee, K.; Heeger, A. J.; Bazan, G. C. Adv. Mater. 2011, 23, 2430.

(309) Kim, J. S.; Lee, Y.; Lee, J. H.; Park, J. H.; Kim, J. K.; Cho, K. Adv. Mater. 2010, 22, 1355.

(310) Wang, L.; Qiao, Z.; Gao, C.; Liu, J.; Zhang, Z.-G.; Li, X.; Li, Y.; Wang, H. Macromolecules 2016.

(311) Frechet, J.; Thompson, B. Angew. Chem. Int. Ed 2008, 47, 58.

(312) Beaujuge, P. M.; Fréchet, J. M. J. Am. Chem. Soc. 2011, 133, 20009.

(313) Li, Y. Acc. Chem. Res. 2012, 45, 723.

(314) Usta, H.; Lu, G.; Facchetti, A.; Marks, T. J. J. Am. Chem. Soc. 2006, 128, 9034. (315) Hou, J.; Chen, H.-Y.; Zhang, S.; Li, G.; Yang, Y. J. Am. Chem. Soc. 2008, 130, 16144. 
(316) Yue, W.; Zhao, Y.; Shao, S.; Tian, H.; Xie, Z.; Geng, Y.; Wang, F. J. Mater. Chem. 2009, 19, 2199.

(317) McCulloch, I.; Heeney, M.; Bailey, C.; Genevicius, K.; MacDonald, I.; Shkunov, M.; Sparrowe, D.; Tierney, S.; Wagner, R.; Zhang, W. Nature materials 2006, 5, 328 .

(318) Beaujuge, P. M.; Pisula, W.; Tsao, H. N.; Ellinger, S.; Müllen, K.; Reynolds, J. R. J. Am. Chem. Soc. 2009, 131, 7514.

(319) Thompson, B. C.; Fréchet, J. M. Angew. Chem. Int. Ed. 2008, 47, 58.

(320) Janietz, S.; Bradley, D.; Grell, M.; Giebeler, C.; Inbasekaran, M.; Woo, E. Appl. Phys. Lett. 1998, 73, 2453.

(321) Dawson, R.; Cooper, A. I.; Adams, D. J. Polym. Int. 2013, 62, 345.

(322) Dawson, R.; Cooper, A. I.; Adams, D. J. Prog. Polym. Sci. 2012, 37, 530.

(323) Kaur, P.; Hupp, J. T.; Nguyen, S. T. ACS Catalysis 2011, 1, 819.

(324) McKeown, N. B.; Budd, P. M. Macromolecules 2010, 43, 5163.

(325) Germain, J.; Fréchet, J. M.; Svec, F. Small 2009, 5, 1098.

(326) Thomas, A. Angew. Chem. Int. Ed. 2010, 49, 8328.

(327) Zhang, Y.; Riduan, S. N. Chem. Soc. Rev. 2012, 41, 2083.

(328) Ben, T.; Ren, H.; Ma, S.; Cao, D.; Lan, J.; Jing, X.; Wang, W.; Xu, J.; Deng, F.; Simmons, J. M. Angew. Chem. 2009, 121, 9621.

(329) Dawson, R.; Laybourn, A.; Clowes, R.; Khimyak, Y. Z.; Adams, D. J.; Cooper, A. I. Macromolecules 2009, 42, 8809. 
(330) Weber, J.; Thomas, A. J. Am. Chem. Soc. 2008, 130, 6334.

(331) Jiang, J. X.; Su, F.; Trewin, A.; Wood, C. D.; Campbell, N. L.; Niu, H.; Dickinson, C.; Ganin, A. Y.; Rosseinsky, M. J.; Khimyak, Y. Z. Angew. Chem. Int. Ed. 2007, 46, 8574.

(332) Jiang, J.-X.; Trewin, A.; Adams, D. J.; Cooper, A. I. Chemical Science 2011, $2,1777$.

(333) Li, P. F.; Schon, T. B.; Seferos, D. S. Angew. Chem. Int. Ed. 2015, 54, 9361. (334) Sprick, R. S.; Jiang, J.-X.; Bonillo, B.; Ren, S.; Ratvijitvech, T.; Guiglion, P.; Zwijnenburg, M. A.; Adams, D. J.; Cooper, A. I. J. Am. Chem. Soc. 2015, 137, 3265. (335) Lim, H.; Chang, J. Y. Macromolecules 2010, 43, 6943.

(336) Facchetti, A.; Vaccaro, L.; Marrocchi, A. Angew. Chem. Int. Ed. 2012, 51, 3520.

(337) Bura, T.; Morin, P.-O.; Leclerc, M. Macromolecules 2015, 48, 5614.

(338) Rudenko, A. E.; Khlyabich, P. P.; Thompson, B. C. ACS Macro Letters 2014, 3,387 .

(339) Luzio, A.; Fazzi, D.; Nübling, F.; Matsidik, R.; Straub, A.; Komber, H.; Giussani, E.; Watkins, S. E.; Barbatti, M.; Thiel, W. Chemistry of Materials 2014, $26,6233$.

(340) Kuwabara, J.; Yasuda, T.; Choi, S. J.; Lu, W.; Yamazaki, K.; Kagaya, S.; Han, L.; Kanbara, T. Advanced Functional Materials 2014, 24, 3226. 
(341) Liu, D.-P.; Chen, Q.; Zhao, Y.-C.; Zhang, L.-M.; Qi, A.-D.; Han, B.-H. ACS Macro Letters 2013, 2, 522.

(342) Zhang, Y.; Zou, J.; Yip, H.-L.; Chen, K.-S.; Davies, J. A.; Sun, Y.; Jen, A. K.Y. Macromolecules 2011, 44, 4752.

(343) Gao, X.; Shen, J.; Hu, B.; Tu, G. Macromol. Chem. Phys. 2014, 215, 1388.

(344) Cardenas, L.; Gutzler, R.; Lipton-Duffin, J.; Fu, C.; Brusso, J. L.; Dinca, L. E.; Vondráček, M.; Fagot-Revurat, Y.; Malterre, D.; Rosei, F. Chemical Science 2013, $4,3263$.

(345) Sing, K. S. Pure Appl. Chem. 1985, 57, 603.

(346) Rose, M.; Klein, N.; Böhlmann, W.; Böhringer, B.; Fichtner, S.; Kaskel, S. Soft Matter 2010, 6, 3918.

(347) Ren, S.; Dawson, R.; Adams, D. J.; Cooper, A. I. Polymer Chemistry 2013, 4, 5585.

(348) Zhang, K.; Kopetzki, D.; Seeberger, P. H.; Antonietti, M.; Vilela, F. Angew. Chem. Int. Ed. 2013, 52, 1432.

(349) Weber, J.; Schmidt, J.; Thomas, A.; Böhlmann, W. Langmuir 2010, 26, 15650. 


\section{APPENDIX}

\section{AI-1. Journal publications}

1. Amsalu Efrem, Kai Wang, Prince N. Amaniampong, Cangjie Yang, Sukriti Gupta, Hassan Bohra, Samir Hemant Mushrif, and Mingfeng Wang. "Direct arylation polymerization towards a narrow bandgap conjugated microporous polymer with hierarchical porosity" Polymer Chemistry 30(2016): 4862-4866.

2. Amsalu Efrem, Marc Courté, Kai Wang, Denis Fichou, Mingfeng Wang. "Synthesis and characterization of $\gamma$-lactone-Pechmann dye based donoracceptor conjugated polymers" Dyes and Pigments 134 (2016): 171-177.

3. Amsalu Efrem, Yanlian Lei, Bo Wu, Mingfeng Wang, Siu Choon Ng, and Beng S. Ong. "Dithienobenzochalcogenodiazole-based electron donor-acceptor polymers for organic electronics." Dyes and Pigments 129 (2016): 90-99.

4. Amsalu Efrem, Chia-Juan Lim, Yong Lu, and Siu-Choon Ng. "Synthesis and characterization of dithienobenzothiadiazole-based donor-acceptor conjugated polymers for organic solar cell applications." Tetrahedron Letters 55, 35 (2014): 4849-4852.

5. Bohra, Hassan, Si Yu Tan, Jinjun Shao, Cangjie Yang, Amsalu Efrem, Yanli Zhao, and Mingfeng Wang. "Narrow bandgap thienothiadiazole-based conjugated porous polymers: from facile direct arylation polymerization to 
tunable porosities and optoelectronic properties." Polymer Chemistry 7, 41

(2016): 6413-6421.

6. Amsalu Efrem, Kai Wang, Tao Jia, Mingfeng Wang. "Direct Arylation Polymerization Towards a Narrow Bandgap Donor-Acceptor Conjugated Polymer of Alternating 5,6-Difluoro-2,1,3-Benzothiadiazole and Alkylquarternarythiophene: From Synthesis, Optoelectronic Properties to Devices". J. Polym. Sci. A Polym. Chem. (2017): Just Accepted.

\section{AI-2. Conference presentations}

1. Amsalu Efrem, Chia-Juan Lim, Yong Lu, Beng S. Ong, Siu-Choon Ng, Mingfeng Wang* "Synthesis and characterization of dithienobenzothiadiazolebased donor- acceptor conjugated polymers for organic electronics application" at $12^{\text {th }}$ International Symposium on Functional $\pi$-Electron Systems. Seattle, USA, 2015 (July 19-24).

2. Amsalu Efrem, Chia-Juan Lim, Yong Lu, Siu-Choon Ng* "Synthesis and Characterization of Dithienobenzothiadiazole-based Donor-Acceptor Conjugated Polymers for Organic Solar Cell Applications" at 6th MRS-S Conference on Advanced Materials, Singapore. 2014 (July 22-25). 
3. Amsalu Efrem, Mingfeng Wang* "Dithienobenzochalcogenodiazole-Based Electron Donor-Acceptor Polymers for Organic Electronics (OFET, OPV)" at SCBE Ph.D. symposium, Singapore. 2016. 